\title{
The Genomic Landscape of Phaeochromocytoma
}

\author{
Presented By \\ Aidan Flynn B.Biomed.Sc(Hons) \\ orcid.org/0000-0002-1933-505X
}

Submitted in total fulfilment of the requirements for the degree of

Doctor of Philosophy

Peter MacCallum Cancer Centre
and
Department of Pathology
The University of Melbourne
Melbourne, Australia

December, 2015

Produced on archival quality paper 


\title{
Declaration
}

The experiments in this thesis constitute work carried out by the candidate unless otherwise stated. The thesis is less than 100,000 words in length, exclusive of tables, figures, bibliography and appendices, and complies with the stipulations set out for the degree of Doctor of Philosophy by the University of Melbourne.

\author{
Aidan Flynn \\ Peter MacCallum Cancer Centre \& \\ Department of Pathology \\ University of Melbourne \\ Parkville, Victoria 3052 \\ Australia
}




\section{Table of Contents}

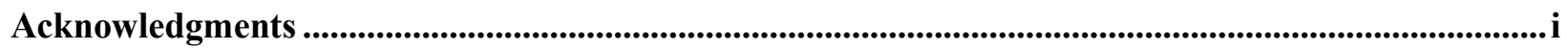

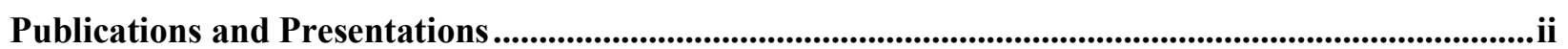

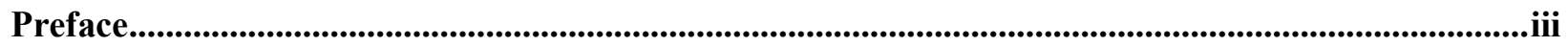

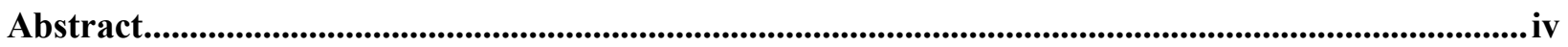

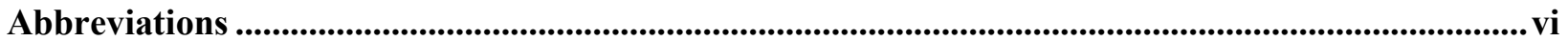

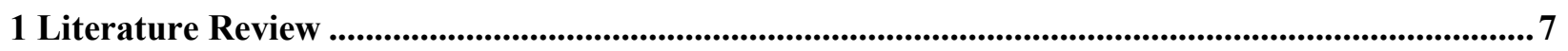

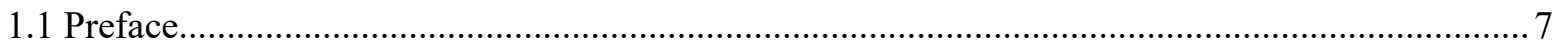

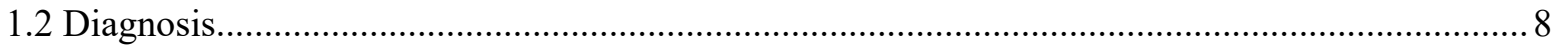

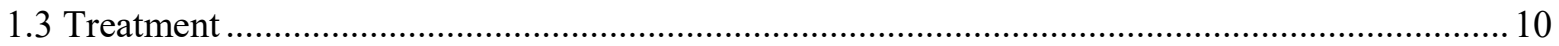

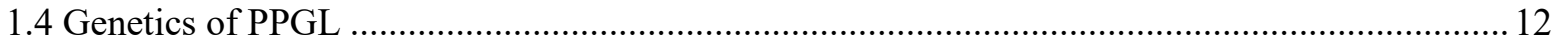

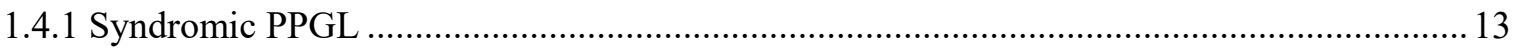

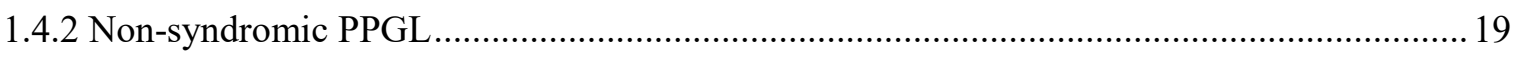

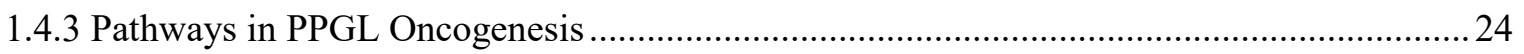

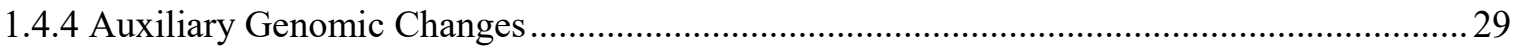

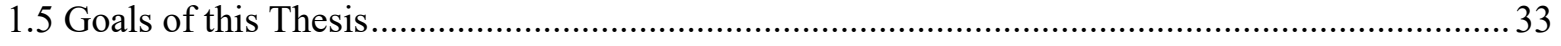

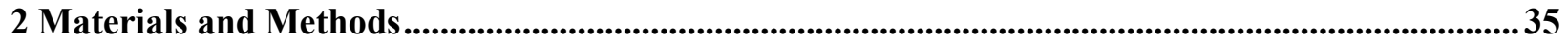

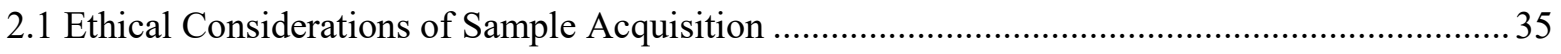

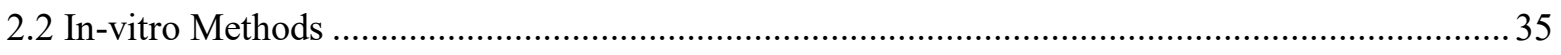

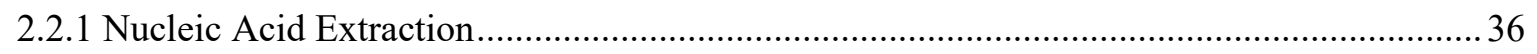

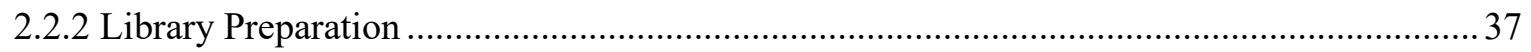

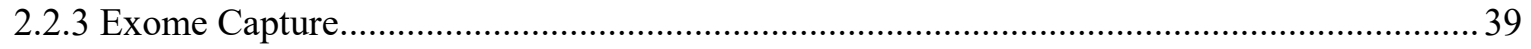

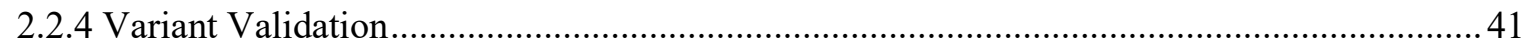

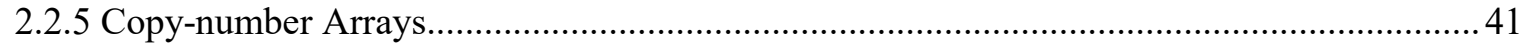

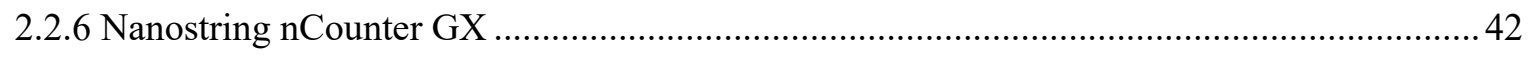

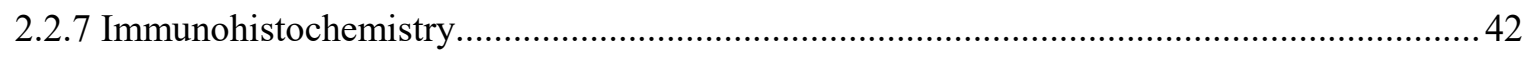

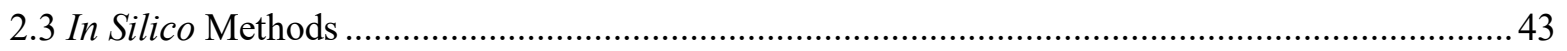




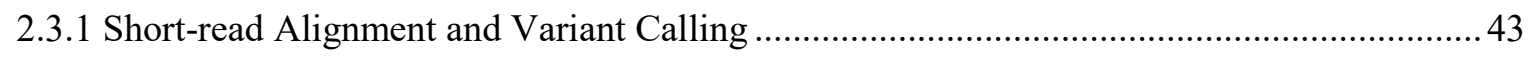

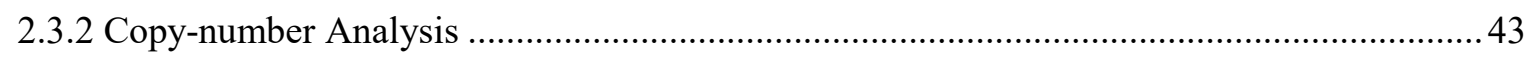

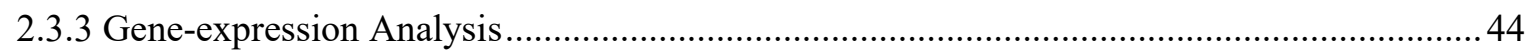

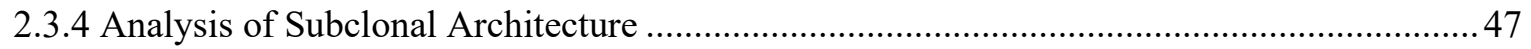

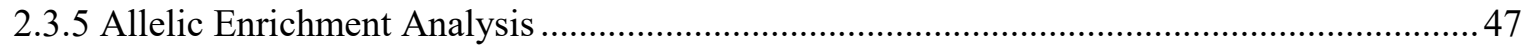

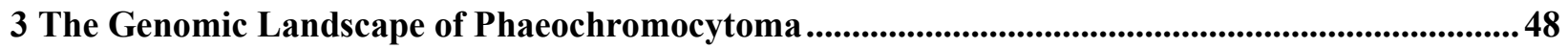

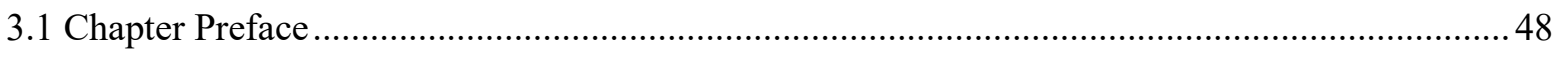

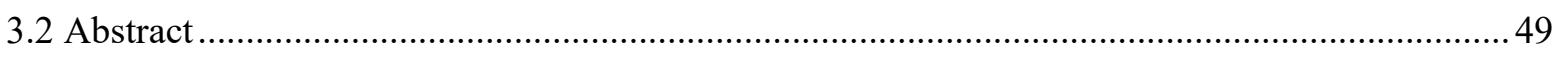

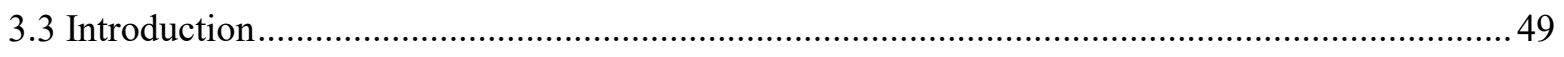

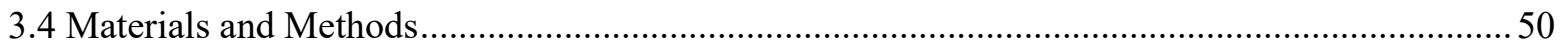

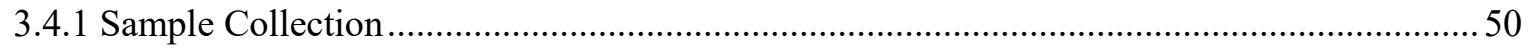

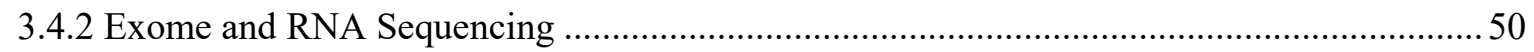

3.4.3 Short-read Alignment and Variant Calling …............................................................... 50

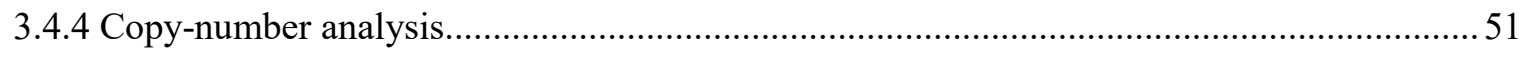

3.4.5 Consensus Clustering, Cross-Platform Classification, and Differential Gene-Expression

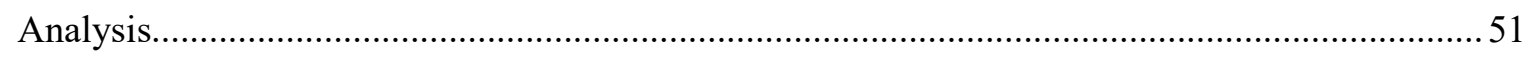

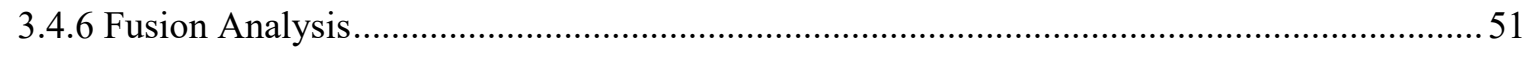

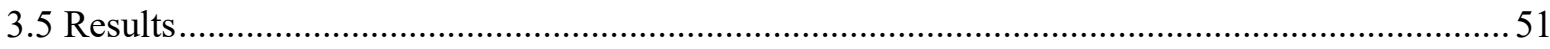

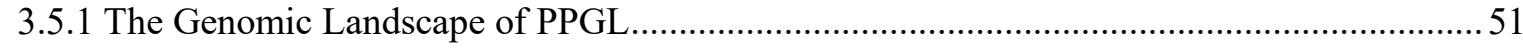

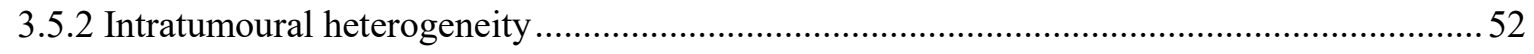

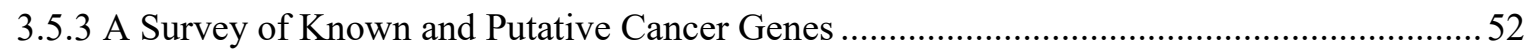

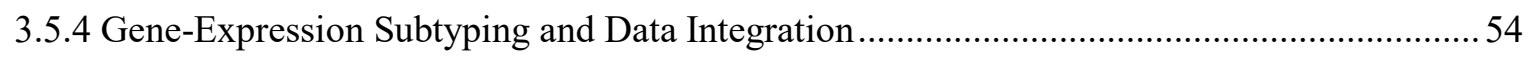

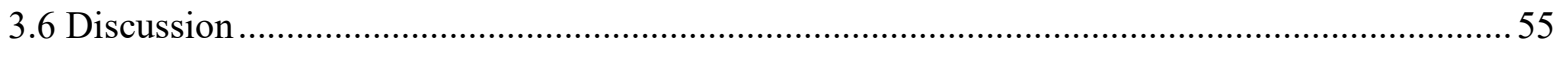

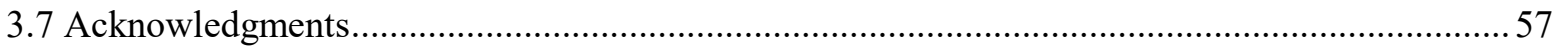

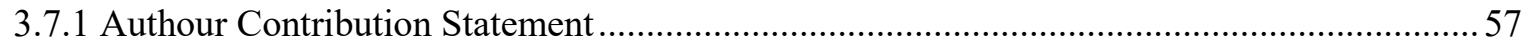

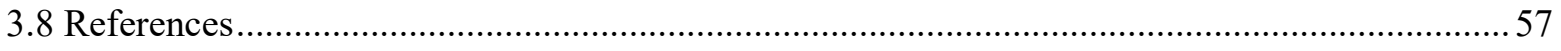


4 A Diagnostic Gene-expression Assay for the Classification of PPGL ............................................61

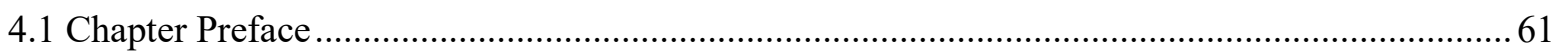

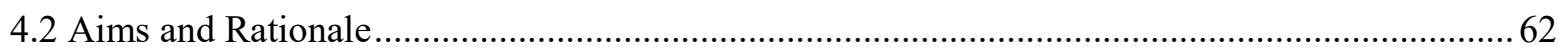

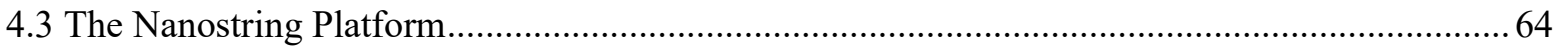

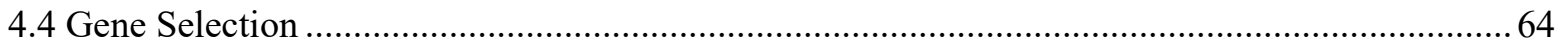

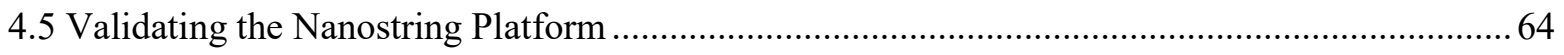

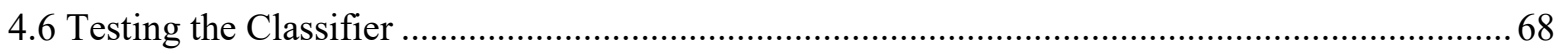

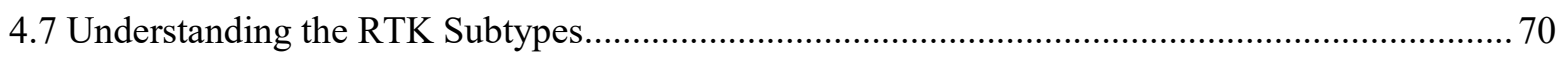

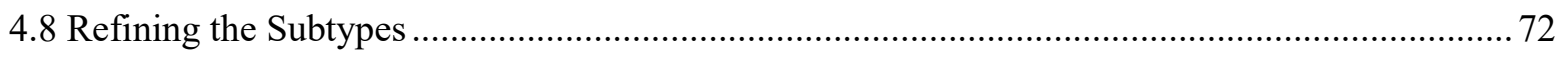

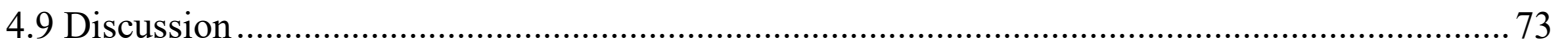

5 Inter- and intra-tumoural heterogeneity in the context of syndromic neuroendocrine tumours... 76

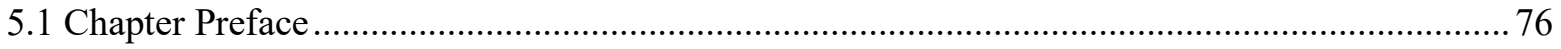

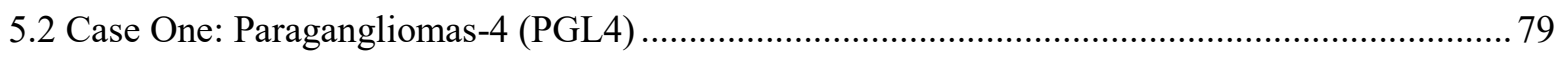

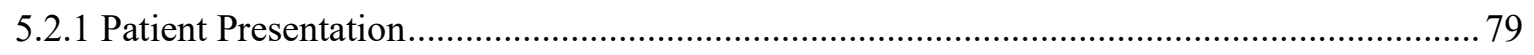

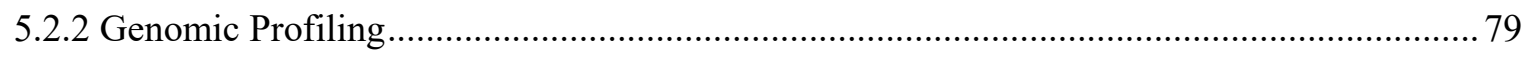

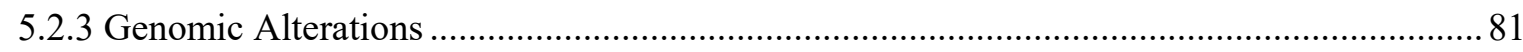

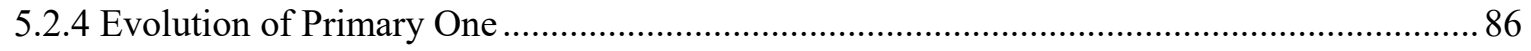

5.3 Case Two: Multiple Endocrine Neoplasia Type IIA (MEN2A) ............................................... 89

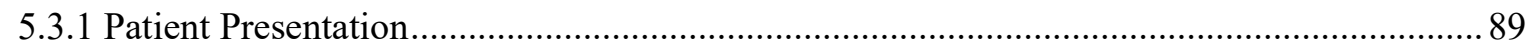

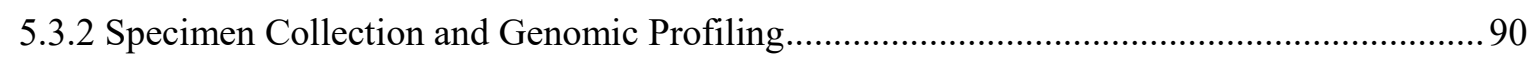

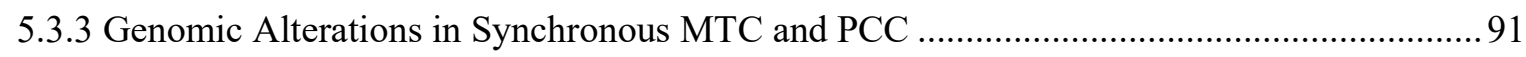

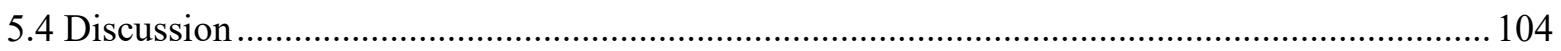

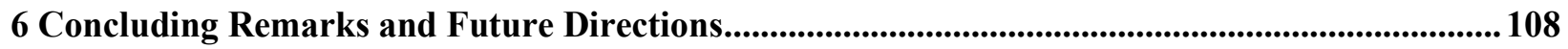

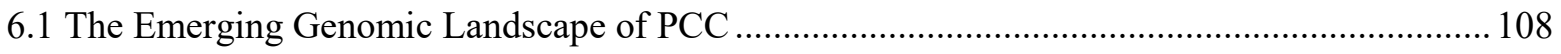

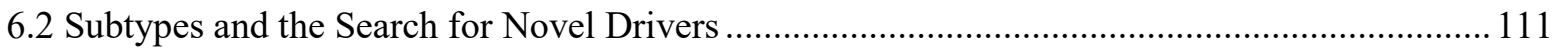

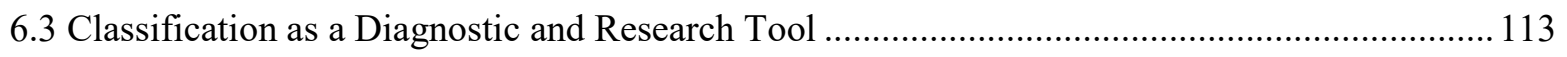

6.4 Cousins not Twins: Lessons from Sub-Clones and Synchronous Primaries ............................. 115 


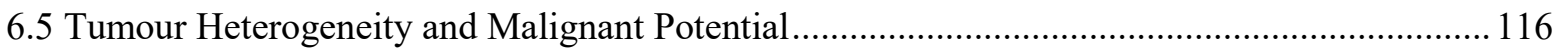

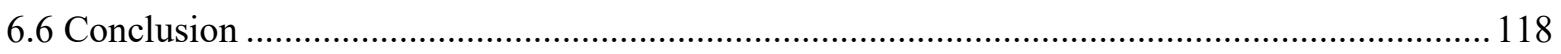

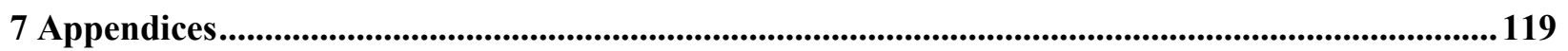

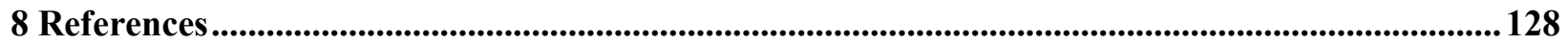




\section{Figures}

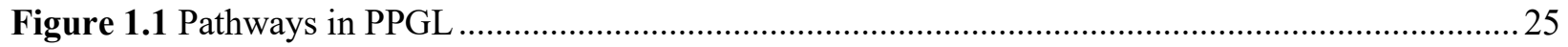

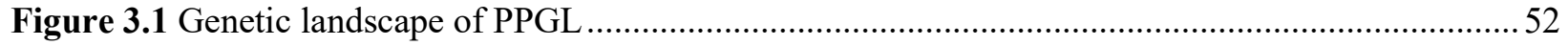

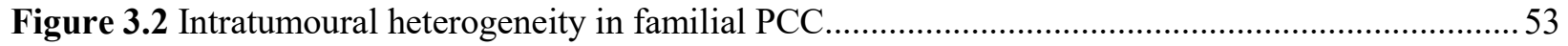

Figure 3.3 Known and putative driver genes detected in PPGL ......................................................... 54

Figure 3.4 Consensus clustering by gene-expression profiling, subtyping, and data integration ..............56

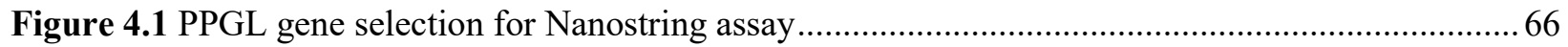

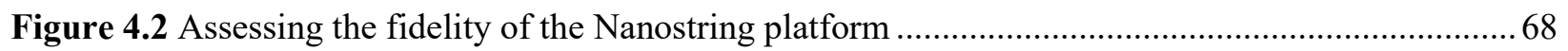

Figure 4.3 Histological heterogeneity in a MAX -driven tumour ........................................................... 70

Figure 4.4 Elimination of RTK2 gene expression through chromogranin-A guided macrodissection...... 71

Figure 4.5 A comparison of classifier models for prediction of PPGL subtypes..................................... 73

Figure 5.1 Anatomical view of synchronous PGL primary tumours ...................................................... 80

Figure 5.2 High resolution copy-number analysis of synchronous PGL ................................................ 82

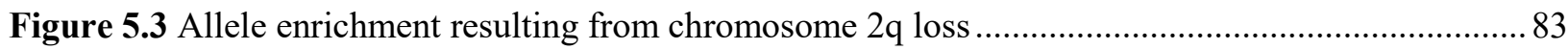

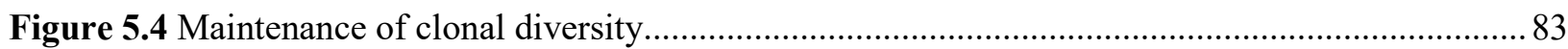

Figure 5.5 Consequence and distribution of mutations in synchronous PGL ......................................... 86

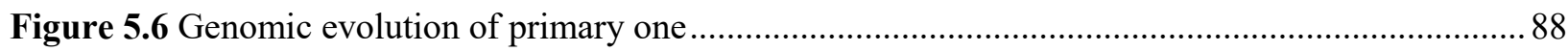

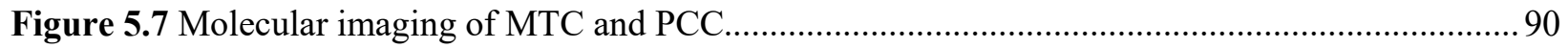

Figure 5.8 Medullary thyroid carcinoma biospecimen collection........................................................ 92

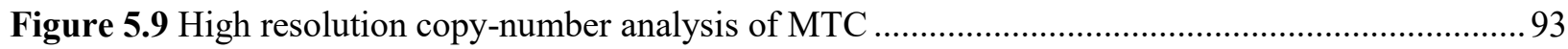

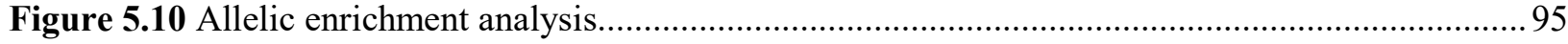

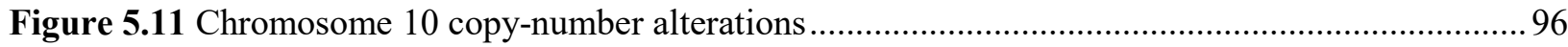

Figure 5.12 Consequence and distribution of mutations in synchronous MTC and PCC (Case 2) .......... 97

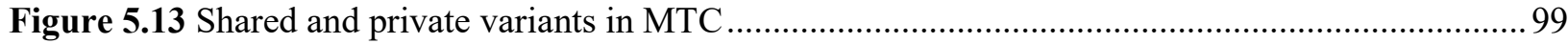

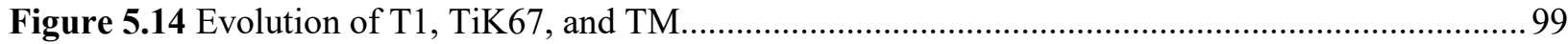

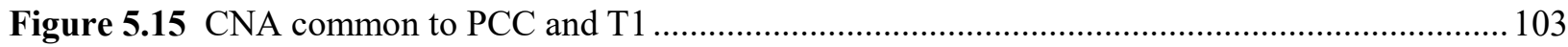

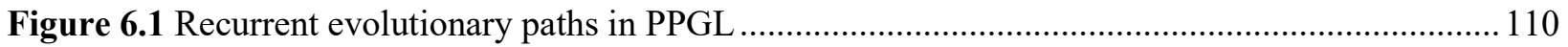




\section{Tables}

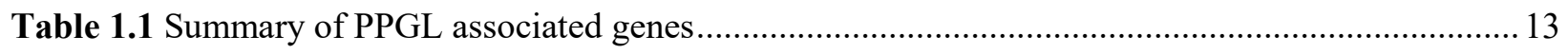

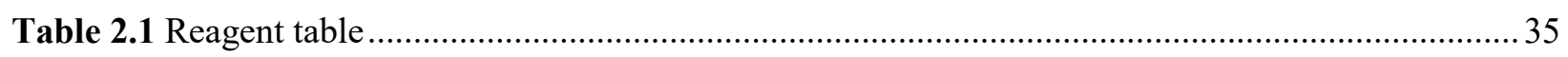

Table 3.1 Common genes and pathways affected by validated somatic lesions in PPGL tumours ...........55

Table 4.1 Correlation matrix of Burnichon et al. (2011) clusters and consensus clustering subtypes ....... 62

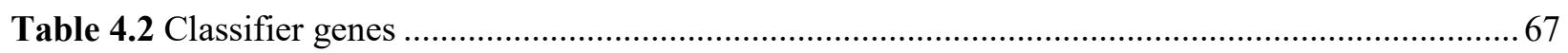

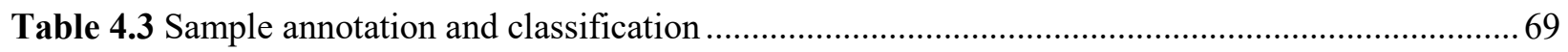

Table 4.4 Number of samples in each class by driver mutation........................................................... 72

Table 5.1 Genes harbouring deleterious mutations enriched by loss of chromosome $2 \mathrm{q}$....................... 84 


\section{Acknowledgments}

Although the title page of this document lists only my name, the reality is that producing a thesis is a little like a Broadway production, without the supporting cast and crew there would be no opening night.

With that in mind, I would like to thank:

My family for supporting me unconditionally in my pursuit of this final stage of my higher education.

Rod Hicks, firstly for giving me the opportunity to begin this journey, secondly for contributing his vast clinical expertise to this phase of my education, and finally for providing the kind of engaging and productive research environment that inspired me to complete the journey.

Annette Hogg for her tremendous effort keeping the wheels from falling off when it came to ethics and sample acquisition.

Joshy George for helping me find my feet with differential expression analysis and machine learning.

Tim Semple, Tim Holloway, Aga Borcz, and Sreeja Gadipally for sharing an office space with me for the last few years, especially considering the chaos that is my desk.

Sally Hunter for being my coffee and whinging buddy.

Mark Shackleton, for keeping my eye on the bigger picture and providing quarterly pushes towards the finish line.

Our collaborators Rory Clifton-Bligh, Diana Benn, and Anthony Gill at the Kolling Institute and Royal Northshore Hospital, whose many contributions were integral to the success of this project.

The surgeons, oncologists, and chemotherapy ward nurses at St Vincent's Hospital, Melbourne, without whom I would likely not be alive to write these words.

And finally, I would like to thank Richard Tothill for being my supervisor, my mentor, and my friend. His unwavering enthusiasm has been the lifeblood of this project. 


\section{Publications and Presentations}

\section{Publications}

Flynn, A., D. Benn, R. Clifton-Bligh, B. Robinson, A. H. Trainer, P. James, A. Hogg, K. Waldeck, J. George, J. Li, S. B. Fox, A. J. Gill, G. McArthur, R. J. Hicks and R. W. Tothill (2015). "The genomic landscape of phaeochromocytoma." Journal of Pathology 236(1): 78-89.

\section{Poster Presentations}

Molecular profiling of Phaeochromocytoma; 25th Lorne Cancer Conference, 2013, Melbourne, Australia

Comprehensive molecular characterization of phaeochromocytoma; Australasian Genomic Technologies Association (AMATA), 2013, Surfers Paradise, Australia

A diagnostic gene-expression assay for the classification of phaeochromocytoma; American Association for Cancer Research annual meeting, 2015, Philadelphia, USA 


\section{Preface}

The work presented herein represents a multi-institute and multidisciplinary collaboration with a multitude of participants, without whom this study would surely never have come to fruition.

Access to a large cohort of clinically annotated phaeochromocytoma and paraganglioma (PPGL) samples was provided by Diana Benn and Roderick Clifton-Bligh of the Kolling Institute of Medical Research, Royal North Shore Hospital, New South Wales. Additional PPGL samples were sourced from the Peter MacCallum Cancer Centre and the Royal Melbourne Hospital in Victoria with the help of Dr Alison Trainer, Dr Paul James, and Dr Henry To. Paediatric PPGL samples were obtained from the Westmead Children's Hospital, NSW, with the assistance of Li Zhou and Daniel Catchpoole. Additional samples were provided by the Victorian Cancer Biobank.

Expert advice on disease pathology was provided by Dr Anthony Gill, Royal North Shore Hospital, New South Wales. Supporting immunohistochemistry was performed by Kelly Waldeck, Peter MacCallum Cancer Centre, Victoria, Australia. Laboratory support was provided by Tim Semple, Tim Holloway, Aga Borcz, and Sreeja Gadipally from the Molecular Genomics Core Facility, Peter MacCallum Cancer Centre, Victoria Australia. Bioinformatics advice was provided by Joshy George, Jackson Laboratory, Farmington, Connecticut, USA.

Project management support was provided by Annette Hogg, Peter MacCallum Cancer Centre, Victoria. 


\begin{abstract}
Phaeochromocytomas (PCC) and paragangliomas (PGL) (collectively PPGL) are rare neural crest-derived tumours originating from adrenal chromaffin cells or extra-adrenal sympathetic and parasympathetic tissues. More than a third of PPGL cases are associated with heritable syndromes involving 18 or more known genes. These genes have been broadly partitioned into two groups based on pseudo-hypoxic and receptor tyrosine kinase (RTK) signalling pathways. Many of these genes can also become somatically mutated, although up to one third of sporadic cases have no known genetic driver. Furthermore, little is known of the genes that co-operate with known driver genes to initiate and drive tumourigenesis. To explore the genomic landscape of PPGL, exome sequencing, high-density SNP-array analysis, and RNA sequencing was applied to 36 PCCs and four PGL tumours. All tumours displayed a low mutation frequency in combination with frequent large segmental copy-number alterations and aneuploidy, with evidence for chromothripsis seen in a single case. Thirty-one of forty $(77.5 \%)$ cases could be explained by germline or somatic mutations or structural alterations affecting known PPGL genes. Deleterious somatic mutations were also identified in known tumour-suppressor genes associated with genome maintenance and epigenetic modulation (e.g. TP53, STAG2, KMT2D). A multitude of other genes were also found mutated that are likely important for normal neuroendocrine cell function (e.g. ASCL1, NCAM1, GOLGA1). In addition, the existing paradigm for gene-expression subtyping of PPGL was further refined by applying consensus clustering to a compendium of previously published microarray data, enabling the identification of six robust gene-expression subtypes and subsequent cross-platform classification of RNA-seq data. The majority of cases in the cohort with no identifiable driver mutation were classified into a gene-expression subtype bearing similarity to $M A X$ mutant PPGL, suggesting there are yet unknown PPGL cancer genes that can phenocopy $M A X$ mutations.
\end{abstract}

The cross-platform classification model was then further refined to develop a 46-gene Nanostring-based diagnostic tool capable of classifying PPGL tumours into gene-expression subtypes. The strong genotype-to-subtype relationship in PPGL makes subtyping a powerful tool that can be used clinically to guide and interpret genetic testing, determine surveillance programs and aid in better elucidation of PPGL biology. In applying the diagnostic assay to a test set of 38 cases, correct classification into one of the six subtypes was achieved for $34(90 \%)$ samples based on the known genotype to gene-expression subtype association. The observation that at least one of the six subtypes is likely defined by the presence of nonneoplastic cells led to further refinement into five, four, and three-class architectures, further improving classification accuracy. 
Increasingly tumour heterogeneity is being recognised as one of the most significant challenges facing modern oncology. Genomically diverse tumour regions create additional complexity in predicting treatment response and metastatic potential through biopsy. Multi-region sampling of multiple synchronous primaries from patients with a predisposing germline mutation was used to explore tumour evolution and heterogeneity in PPGL and concomitant medullary thyroid carcinoma. Evolutionary reconstruction of a single primary PPGL demonstrated periods of both branched and linear evolution resulting in a high degree of intratumoural heterogeneity. Comparison of multiple synchronous primaries provided strong evidence of convergent evolution through recurrent chromosomal aberrations, indicating these may be obligate events in tumourigenesis, and as such, may indicate potential novel therapeutic targets. 


\section{Abbreviations}

\begin{tabular}{|c|c|}
\hline INDEL & Insertion or Deletion \\
\hline VUS & Variant of Unknown Significance \\
\hline PCC & Phaeochromocytoma \\
\hline PGL & Paraganglioma \\
\hline PPGL & Phaeochromocytoma and Paraganglioma \\
\hline SDH & Succinate Dehydrogenase \\
\hline PHD & Prolyl Hydroxylase \\
\hline VHL & Von Hippel-Lindau \\
\hline TCA & Tricarboxylic Acid \\
\hline HIF & Hypoxia Indicuble Factor \\
\hline JMJD & Jumonji Histone Demethylases \\
\hline$\alpha-K G$ & $\alpha$-Ketoglutarate \\
\hline PGL1-PGL4 & Paraganglioma Syndrome 1-4 \\
\hline GIST & Gastrointestinal Stromal Tumours \\
\hline GEF & Guanadine Exchange Factor \\
\hline NGF & Nerve Growth Factor \\
\hline HRE & HIF-Responsive Elements \\
\hline VEGF & Vascular Endothelial Growth Factor \\
\hline MAPK & Mitogen-Activated Protein Kinase \\
\hline ALT & Alternate Lengthening of Telomeres \\
\hline GTF & Gene Transfer Format \\
\hline ACA & Adrenal Cortical Adenoma \\
\hline RCC & Renal Cell Carcinoma \\
\hline $\mathrm{SCLC}$ & Small Cell Lung Cancer \\
\hline $\mathrm{CNLOH}$ & Copy-Neutral Loss of Heterozygosity \\
\hline CSG & Cancer Susceptibility Genes \\
\hline CoSMiC & Catalogue of Somatic Mutations in Cancer \\
\hline TCGA & The Cancer Genome Atlas \\
\hline
\end{tabular}




\section{Literature Review}

\subsection{Preface}

The human body is formed of a multitude of cell types performing unique and necessary functions. These functions need to be coordinated over both short and long intervals, a process achieved by intercellular signalling. In addition to localised intercellular signalling, signals are transmitted between remote organ systems either through the nervous system as electrical potential or as signalling molecules released by the endocrine system and carried in the blood. There is an obvious need for the nervous and endocrine systems to be integrated to form a seamless communication system throughout the body; this system is termed the neuroendocrine system.

Chromaffin cells are a specialised type of neuroendocrine cell that arise from progenitor cells in the neural crest, the largest population of which is located in the medulla of the adrenal gland. Extraadrenally, the organ of Zuckerkandl represents the largest accumulation of chromaffin cells, while smaller deposits, known as chromaffin bodies or chromaffin paraganglia, can be found associated with the ganglia of the sympathetic trunk. These specialist cells are responsible for the production and secretion of the catecholamines, epinephrine and norepinephrine, and play an integral role in the "fight or flight" response. Under basal conditions small amounts of catecholamines are constantly released into the blood, but in situations of physical exertion or stress the concentration can be rapidly increased up to 10 -fold (Cryer, 1980; Takiyyuddin et al., 1994). Upon release, catecholamines enact physiological changes including elevated heart rate, increased blood pressure, and a rise in blood glucose to ensure adequate blood and energy supply to major organs.

Chromaffin cells, like most cell types, are vulnerable to changes in their genetic program leading to uncontrolled growth and proliferation. When this occurs in chromaffin cells of the adrenal medulla the resulting tumour is known as a phaeochromocytoma, at extra-adrenal sites the terms paraganglioma and extra-adrenal phaeochromocytoma are used interchangeably. The paraganglioma nomenclature is also given to a highly similar but distinct tumour arising from non-chromaffin glomus cells. These cells, also of neural crest origin, act as specialised chemoreceptors predominantly involved in oxygen sensing. In the interest of clarity, this review will use the terms phaeochromocytoma (PCC) and paraganglioma (PGL) to refer to tumours of chromaffin origin, whilst the term parasympathetic paraganglioma will be used for tumours of non-chromaffin origin. For convenience, the term PPGL will encompass both PCC and PGL. 


\subsection{Diagnosis}

PPGL is a relatively rare neoplasm with an annual incidence between 2 and 8 per 1,000,000 people (Beard et al., 1983; Stenstrom \& Svardsudd, 1986). These tumours represent a diagnostic challenge as they often exhibit limited or ambiguous symptoms. Headaches, sweating, and palpitations are among the most common (Lenders et al., 2005), with many patients exhibiting paroxysmal or sustained hypertension (Mazza et al., 2014). Other symptoms include tachycardia, pallor, headache, feelings of panic or anxiety, and less frequently, nausea, fever, and flushing (Martucci \& Pacak, 2014). The majority of these symptoms are a result of the secretion of excess catecholamines, however, not all PPGL remain functional or produce the same catecholamine profile. Therefore symptoms may vary widely between patients or be entirely absent. One retrospective study of forty-one patients found that around half of the included PPGL were discovered during routine imaging for an unrelated condition (Baguet et al., 2004). The rare nature of PPGL precludes any systematic screening on a population level as is done for cancers of the breast, colon, and prostate. In individuals with a family history of PPGL, or a positive genetic test for mutations in a known susceptibility gene, regular screening is recommended.

As a significant proportion of PPGL are functional, secretory products released by PPGL provide useful diagnostic markers. In chromaffin cells, catecholamines are synthesised in the cytoplasm and sequestered into vesicles by vesicular monoamine transporters (Henry et al., 1994). However, the vesicles are leaky and catecholamines slowly diffuse back into the cytoplasm where they are metabolised by catechol-Omethyltransferase to form metanephrine and normetanephrine. These metabolites can be directly eliminated in the urine or further metabolised in the liver to form Vanillylmandelic acid. Historically, diagnostic methods relied on measurements of urinary or plasma catecholamines. However, many tumours exhibit fluctuating levels of catecholamine release, which can lead to false negative results (Martucci et al., 2014). Metabolism of catecholamines in the adrenal medulla accounts for $91 \%$ of free metanephrine and $23 \%$ of normetanephrine in the blood of healthy individuals (Eisenhofer et al., 1995) and, as such, are highly specific markers for the metabolic activity of chromaffin cells. A comparison of detection methods showed plasma free metanephrines to have the highest sensitivity (99\%) and urinary VMA to has the lowest (66\%), however, urinary VMA has the highest specificity (95\%) while urinary fractionated metanephrines have the lowest (69\%) (Lenders et al., 2002). The authors concluded that plasma free metanephrines provided the best combination of sensitivity $(99 \%)$ and specificity (89\%) and that combining multiple tests did not improve the detection accuracy.

Chromogranin $\mathrm{A}(\mathrm{CgA})$ is also an established biomarker used in the detection of neuroendocrine tumours. $\mathrm{CgA}$ is a precursor peptide to three biologically active peptides; vasostatin, pancreastatin, and parastatin. 
These peptides act through autocrine and paracrine mechanisms to regulate the neuroendocrine system. While CgA is not specific to PPGL, evidence suggests that in combination with metanephrine assays, it provides increased detection sensitivity (d'Herbomez et al., 2007; Zuber et al., 2014).

Where available, molecular imaging provides an important diagnostic tool for PPGL. Modern imaging techniques leverage specific biochemical features of PPGL to achieve a high sensitivity and specificity. Metaiodobenzylguanidine (MIBG) is a synthetic analogue of norepinephrine that is sequestered through catecholamine symporters and subsequently stored in neurosecretory granules (Wafelman et al., 1994). In diagnostic applications, MIBG is radiolabelled with Iodine $\left({ }^{123} \mathrm{I}-\mathrm{MIBG} /{ }^{131} \mathrm{I}-\mathrm{MIBG}\right)$ and imaged using single-photon emission computed tomography (SPECT). As MIBG targets norepinephrine storage granules, the primary clinical indication for usage of MIBG scintigraphy is in catecholamine producing tumours such as functional PPGL, neuroblastomas, and medullary thyroid carcinomas (Rufini et al., 2006).

68Ga-DOTA-octreotate (GaTate), a positron emitting radiotracer comprised of the somatostatin analogue octreotate conjugated to Gallium $\left({ }^{68} \mathrm{Ga}\right)$ can be used to selectively target cells expressing the somatostatin receptor (SSTR). As overexpression of the somatostatin receptor is recognised as a distinctive feature of some neuroendocrine tumours (Fischer et al., 2008; Oberg, 2005; Schulz et al., 2000). Recent research suggests that GaTate provides superior sensitivity and specificity when compared with a range of other imaging modalities in the detection of PPGL (Janssen et al., 2015).

In patients presenting with somatostatin or MIBG negative disease, the positron emitting radiopharmaceutical ${ }^{18} \mathrm{~F}$-fluorodeoxyglucose $\left({ }^{18} \mathrm{~F}\right.$-FDG) provides significant diagnostic utility (Timmers et al., 2007). ${ }^{18} \mathrm{~F}$-FDG is a glucose molecule on which one hyroxyl group has been substituted with radioactive Fluorine $\left({ }^{18} \mathrm{~F}\right) .{ }^{18} \mathrm{~F}-\mathrm{FDG}$ is sequestered by cells in place of glucose through GLUT transporters where it becomes trapped following phosphorylation by a hexokinase (Carrasquillo \& Chen, 2010). Some studies have suggested that FDG avidity may have predictive value in determining malignant potential, although it is difficult to discern whether this is independent of the presence of an $S D H B$ mutation, itself a risk factor of malignancy (Fikri et al., 2014).

PPGL have a predominantly benign indolent progression, however, a portion develop metastases to unrelated sites including lymph nodes, bone, lungs, and liver. The proportion of PPGL that become metastatic remains in contention, historically it has been considered to be around 10\% (Eisenhofer et al., 2004). However, recent studies have suggested figures between $15 \%$ and $25 \%$ (Ayala-Ramirez et al., 
2011; Jimenez et al., 2013). The current World Health Organisation guidelines on PPGL do not recognise any clinical or histopathological features that can differentiate benign from metastatic PPGL in the absence of disseminated disease. Despite this, three clinical predictors of increased likelihood of metastasis have been reported; primary tumour location, tumour size, and a germline mutation in the $S D H B$ subunit.

In order to assess the relationship between site of origin and metastatic potential Park et al. (Park et al., 2011) examined a series of 152 benign and malignant PPGL. Overall, adrenal PCC accounted for 137 (90.1\%) and PGL 15 (9.8\%) of the 152 cases, despite this imbalance, PGL accounted for $4(23.6 \%)$ of the 17 metastatic tumours. Other studies examining metastatic potential have shown that a tumour size larger than $5 \mathrm{~cm}$ is significantly correlated with increased risk of metastasis and decreased overall survival duration (de Wailly et al., 2012; Park et al., 2011). Furthermore, a large retrospective study encompassing 365 PPGL patients, 105 of which had metastatic disease, demonstrated that despite their correlation, tumour size, tumour location, and SDHB mutation are independent risk factors for malignancy(Eisenhofer et al., 2012).

In 2002, a scoring algorithm called the Phaeochromocytoma of the Adrenal gland Scaled Score (PASS) was proposed to differentiate benign from malignant disease (Thompson, 2002). The study reviewed histological features of fifty benign and fifty malignant cases and assigned a value of one or two to each feature giving a cumulative score to each tumour based on the features present. The study concluded that all benign tumours studied had a PASS less than four while all malignant tumours scored greater than four, although the author does add the caveat that these values are not absolute indicators. Despite becoming widely used within the field, more recent systematic reviews of the method have had mixed results. Detractors cast doubt on the utility of the metric citing both poor prognostic value and a potential for observer bias (Agarwal et al., 2010; de Wailly et al., 2012; Gao et al., 2006; Wu et al., 2009).

\subsection{Treatment}

In cases of benign PPGL, standard clinical management involves the pharmacological treatment of symptoms associated with catecholamine excess followed by surgical excision of the primary tumour. Proper management of catecholamine associated symptoms both before and during surgery is of the utmost importance to prevent hypertensive crisis. This is usually achieved through the administration of $\alpha$-adrenergic receptor blockers and calcium channel antagonists. In severe cases additional treatment with $\alpha$-methyl paratyrosine to inhibit catecholamine synthesis may be necessary (Tada et al., 1998). In cases where complete surgical resection is not possible or metastatic disease is evident ${ }^{131}$ I-MIBG therapy has 
been the mainstay of treatment but novel therapies such as peptide receptor radionuclide therapy are also being employed.

As mentioned previously, MIBG is a norepinephrine analogue that is sequestered by various neuroendocrine cell types. The same characteristics of radiolabelled MIBG that facilitate molecular imaging of PPGL also make it a good candidate radiotherapeutic agent. Clinical indication for use of ${ }^{131} \mathrm{I}-$ MIBG therapy is non-responsive, aggressive metastatic disease with strong positive imaging using ${ }^{123}$ IMIBG or ${ }^{124}$ I-MIBG as a tracer. In 1997, a review of 116 patients who received MIBG therapy found that patients who had an initial response to MIBG had a lower rate of mortality (33\%) compared to nonresponders (45\%) and an increased survival time (median 22 months versus 13 months) at follow-up. However, complete remission was only seen in $4 \%$ of patients (Loh et al., 1997). A more recent metaanalysis of 17 studies encompassing 243 patients found MIBG therapy resulted in complete or partial tumour response, or stable disease in 3\%, 27\%, and 52\% of patients respectively (van Hulsteijn et al., 2014). These results suggest that while MIBG therapy is not curative in the majority of cases, it does provide an increase in progression free survival and palliative relief from disease associated morbidity.

Traditional external beam radiotherapy applies ionising radiation from an external source, which has to pass through any intervening healthy tissue to reach the therapeutic target leading to unwanted tissue damage. Peptide Receptor Radionuclide Therapy (PRRT) employs a radionuclide such as lutetium $\left({ }^{177} \mathrm{Lu}\right)$, yttrium $\left({ }^{90} \mathrm{Y}\right)$, or indium $\left({ }^{111} \mathrm{In}\right)$ conjugated to a receptor-specific peptide via a chelating agent such as DOTA (1,4,7,10-tetraazacyclododecane-1,4,7,10-tetraacetic acid). The PRRT agent is then introduced to the patient's blood stream where the circulating radionuclide complex binds to a reciprocal receptor on the surface of the tumour cells, delivering the radiation dose directly to the tumour. The remaining circulating PRRT agent is rapidly cleared by the kidneys. Although PRRT has shown potential in other neuroendocrine tumours (van Essen et al., 2009), there is very limited information on its efficacy in PPGL. Small studies with between 12 and 40 patients have seen partial tumour responses in $36 \%$ of PCC and between 10 and 25\% of PGL (Imhof et al., 2011; van Essen et al., 2006). While further studies are required, these preliminary results suggest that PRRT may be of some therapeutic benefit in cases that present with poor I-MIBG uptake but image well with somatostatin analogues.

The most widely applied chemotherapeutic regimen for malignant PPGL is a combination therapy with cyclophosphamide, vincristine, and dacarbazine (CVD) first described in 1985 (Keiser et al., 1985). A 22year follow-up study found that while CVD produced a complete response in $11 \%$ and a partial response in $44 \%$ of patients, there was no overall difference in survival between the responders and non- 
responders. Even so, patients exhibiting tumour response reported a reduction in catecholamine related symptoms and improvements in hypertension. As such, the therapy is generally considered palliative rather than curative.

As the molecular mechanisms of oncogenesis become increasingly understood, there has been a greater focus on the use of targeted therapies. Sunitinib is a multi-target receptor tyrosine kinase inhibitor with antiangiogenic and antitumour activity. Targets of sunitinib include the PDGF-Rs, VEGF-Rs, and RET. Recognition of the highly vascularised nature of some PPGLs and the underlying activation of HypoxiaInducible Factor (HIF) signalling provided the rationale for its application in PPGL. Xenograft models using rat PCC cell line PC12 demonstrated a significant reduction in tumour growth rate following treatment with sunitinib (Denorme et al., 2014). In humans, most published literature to date is limited to single case studies or small cohorts (Hata et al., 2014; Jimenez et al., 2009; Joshua et al., 2009). Initial data from these limited trials suggest sunitinib provides clinical benefit and, as such, sunitinib is now the focus of an international clinical trial (FIRSTMAPPP, clinicaltrials.gov identifier: NCT01371201). Everolimus, an mTORC1 inhibitor, was trialled in a cohort of four patients with limited success, all patients showed disease progression following treatment (Druce et al., 2009). It is postulated that these tumours may be escaping through compensatory PI3K/AKT and ERK activation, as such, research is currently underway into dual-specificity PI3K/mTORC1 inhibitors (Nolting \& Grossman, 2012).

As it stands, there are no highly effective treatments for malignant PPGL. The investigation into novel targeted therapies is still in its infancy and is hampered by the paucity of knowledge surrounding the molecular mechanisms of both oncogenesis and metastasis. It is likely that future treatments will come as a result of greater understanding of the genetic, epigenetic, and micro-environmental changes that foster malignant progression.

\subsection{Genetics of PPGL}

Perhaps one of the most interesting features of PPGL is the relatively high contribution of heritability to its aetiology. Historically, around $10 \%$ of PPGLs were considered to be the result of a germline mutation. However, the last decade and a half has seen a steady expansion in the number of genes linked to PPGL from three to more than eighteen (Favier et al., 2014). It is now estimated that up to $40 \%$ of cases are due to an underlying germline mutation, and a further one-third of sporadic cases can be explained by a somatic mutation in one of the known drivers (Dahia, 2014). Due to the heritable nature of these mutations, PPGL often presents as part of a syndrome in the presence of other tumour types and nontumour related symptoms. 
Table 1.1 Summary of PPGL associated genes

\begin{tabular}{|c|c|c|c|c|c|}
\hline Gene & Pathway & Predominant Site & $\begin{array}{l}\text { Risk of } \\
\text { Malignancy }\end{array}$ & $\begin{array}{l}\text { Related } \\
\text { Conditions }\end{array}$ & $\begin{array}{l}\text { Incidence } \\
\text { Proportion }\end{array}$ \\
\hline VHL & HIF & PCC $>$ PGL & Low & $\begin{array}{l}\text { RCC, } \\
\text { CNS-HAB }\end{array}$ & $9-13 \%$ \\
\hline EPAS1 & HIF & PGL $>$ PCC & Unknown & Polycythemia & $2-5 \%$ \\
\hline EGLN1 & HIF & PGL (Single case) & Unknown & & $<1 \%$ \\
\hline SDHA & TCA/HIF & PGL & Unknown & GIST & $<1 \%$ \\
\hline SDHB & TCA/HIF & PGL $>$ PCC & High & GIST & $7 \%$ \\
\hline SDHC & TCA/HIF & PGL & Low & GIST & $1-2 \%$ \\
\hline SDHD & TCA/HIF & PGL $>$ PCC & Moderate & GIST & $5 \%$ \\
\hline SDHAF2 & TCA/HIF & PGL & Unknown & & $<1 \%$ \\
\hline IDH1 & TCA/HIF & PGL (Single case) & Unknown & & $<1 \%$ \\
\hline $\mathrm{FH}$ & TCA/HIF & $\mathrm{PCC}>\mathrm{PGL}$ & Unknown & $\begin{array}{l}\text { Uterine } \\
\text { leiomyoma }\end{array}$ & $<1 \%$ \\
\hline $\mathrm{MDH} 2$ & TCA/HIF & PGL (Single case) & Unknown & & $<1 \%$ \\
\hline MAX & MYC & $\mathrm{PCC}>\mathrm{PGL}$ & Low & & $1-2 \%$ \\
\hline RET & RAS & PCC & Low & MTC & $5 \%$ \\
\hline NF1 & RAS & PCC $>$ PGL & Moderate & $\begin{array}{l}\text { Neurofibroma, GIST, } \\
\text { optic glioma }\end{array}$ & $15-20 \%$ \\
\hline HRAS & RAS & $\mathrm{PCC} / \mathrm{PGL}$ & Unknown & & $7 \%$ \\
\hline BRAF & RAS & PCC (Single case) & Unknown & & $<1 \%$ \\
\hline TMEM127 & RAS/mTOR & PCC & Low & & $1-2 \%$ \\
\hline KIF1Bbeta & $\begin{array}{l}\text { Neuronal } \\
\text { apoptosis }\end{array}$ & PCC (limited cases) & Unknown & & $<1 \%$ \\
\hline MEN1 & $\begin{array}{l}\text { Epigenetic } \\
\text { control }\end{array}$ & PCC & Unknown & $\begin{array}{l}\text { Tumors of the } \\
\text { parathyroids, } \\
\text { pancreatic islets, } \\
\text { and anterior pituitary }\end{array}$ & $<1 \%$ \\
\hline
\end{tabular}

\subsubsection{Syndromic PPGL}

\subsubsection{Multiple Endocrine Neoplasia Type 1}

Multiple Endocrine Neoplasia Type 1 (MEN1) (MIM: 131100) is an autosomal dominant cancer syndrome giving rise to tumours of the parathyroid glands, pancreatic islet cells, and the anterior pituitary, and less frequently, gastric carcinoids, adrenocortical adenomas, lipomas, angiofibromas, thyroid adenomas, and PPGL (Chandrasekharappa et al., 1997). In 1988, the gene responsible for MEN1 was mapped to chromosome 11 by Catharina Larsson and co-workers, this mapping was further refined through positional cloning to 11q13 in 1997 (Chandrasekharappa et al., 1997; Larsson et al., 1988). The product of the MEN1 gene codes for 67-kDa protein, dubbed menin, with no significant homology to any 
other proteins. Although the function of menin is poorly understood, it is thought to have a role in transcriptional regulation and has been shown to facilitate histone methyltransferase activity when complexed with mixed-lineage leukemia histone methyltransferase $(K M T 2 A)$ and lens epithelium derived growth factor (PSIP1) (Yokoyama \& Cleary, 2008). At present, more than 300 unique mutations have been described in the MEN1 gene (Klein et al., 2005). Disease penetrance in carriers of a pathogenic mutation is estimated to be greater than $90 \%$ by 50 years of age (Trump et al., 1996), however, PPGL occur rarely within the context of MEN1.

\subsubsection{Multiple Endocrine Neoplasia Type 2}

The RET (Rearranged during Transfection) proto-oncogene was discovered in 1985 by Masahide Takahashi and colleagues while transfecting NIH-3T3 cells with human lymphoma DNA. The RET gene product is a transmembrane receptor tyrosine kinase which, in concert with GFR $\alpha$ co-receptors, responds to growth factors of the glial cell line-derived neurotrophic factor (GDNF) family (Arighi et al., 2005). Upon activation, two RET monomers dimerise and undergo autophosphorylation via their intracellular kinase domains (Eng, 1999). Depending on which tyrosine residues become phosphorylated, RET can induce downstream signalling via the PI3K/AKT, RAS/ERK, and RAC/JNK pathways (Murakumo et al., 2006; Prazeres et al., 2011). In mouse knockout models RET has been shown to be essential for nervous system and kidney development, with Ret-/- mice dying shortly after birth (Enomoto et al., 2001; Rozen et al., 2009).

In humans, the effect of RET mutations depends on whether they are loss or gain-of-function mutations. Loss-of-function mutations are associated with Hirschsprung disease, a disorder in which the intrinsic ganglion cells in the myenteric and submucosal plexuses of the gastrointestinal tract do not develop (Eng \& Mulligan, 1997). Gain-of-function mutations are associated with Multiple Endocrine Neoplasia Type 2, which can be further stratified into MEN2A (MIM: 171400), MEN2B (MIM: 162300), and familial medullary thyroid carcinoma (MTC) (MIM: 155240). MEN2A is characterised by the development of PPGL and hyperplasia of the calcitonin producing para-follicular C cells of the thyroid leading to MTC. MEN2B presents a similar phenotype to MEN2A with additional developmental defects and a tendency towards more aggressive tumour progression (Brandi et al., 2001). There is a strong genotype to phenotype correlation in RET related disorders with a handful of point mutations in exons 10 and 11 being responsible for the MEN2A phenotype, and 93\% of MEN2B cases being attributed to a single point mutation in exon 16 (M918T) (Eng et al., 1997; Eng et al., 1994; Mulligan et al., 1994). More recently, somatic mutations in the RET gene have been shown to contribute to the development of PPGL, these mutations occur mostly in accordance with the oncogenic hotspot mutation profile affecting amino acids 
634 and 918 (Burnichon et al., 2011). Overall RET mutations account for around 5\% of PPGL (Brito et al., 2014; Dahia, 2014).

\subsubsection{Neurofibromatosis Type 1}

Neurofibromatosis Type 1 (NF1) (OMIM: 162200) is an autosomal dominant, multi-system disorder with a diverse phenotypic presentation including both tumour and non-tumour manifestations. While neurofibroma, a benign tumour arising from the nerve sheath, is the tumour-type most commonly associated with NF1, patients are also at risk of developing juvenile myelomonocytic leukemia, gastrointestinal stromal tumour, optic glioma, and PPGL (Boyd et al., 2009; Korf, 2000). NF1 has the highest rate of de novo events of any single-gene disorder with approximately half of affected individuals having no affected parent (Theos \& Korf, 2006).

The NF1 gene, which has 60 exons dispersed over more than 300 kilobases of chromosome 17q11.2, encodes neurofibromin and is expressed in a variety of cell types including neurons, glial cells, and Schwann cells (Daston et al., 1992). The gene product contains a RAS-specific guanosine triphosphatase (GTPase)-activating protein domain, which enhances the conversion of RAS-GTP to the inactive form, RAS-GDP, resulting in a decrease of signalling through the RAS signalling cascade (Ballester et al., 1990; Khosravi-Far \& Der, 1994). The role of NF1 as a tumour-suppressor gene is supported by mutational profile surveys demonstrating the absence of mutational hotspots and a preponderance $(\sim 75 \%)$ of mutations predicted to result in a protein truncation event. In addition, loss of heterozygosity at the NF1 locus has been observed in $20-50 \%$ of cutaneous neurofibromas, $70 \%$ of plexiform neurofibromas, and $85 \%$ of PPGL (Ko et al., 2013; Laycock-van Spyk et al., 2011).

Recently, two studies examining sporadic PPGL found that somatic alterations to NF1 were a major contributor to these tumours. One study examining 61 sporadic tumours preselected to exclude mutations in other causative genes demonstrated that 25 cases (41\%) could be explained by somatic alteration in the NF1 gene (Burnichon, Buffet, et al., 2012). The second study which preselected cases exhibiting loss of chromosome 17q (11 of 42 cases studied) demonstrated that 10 of 11 cases harboured somatic NF1 mutations (Welander et al., 2012). Overall, Neurofibromatosis Type 1 accounts for around 5\% of PPGL, whilst somatic mutations in the NF1 gene are thought to account for around $20-25 \%$ of sporadic PPGL (or between 15-20\% of all PPGL) (Dahia, 2014). 


\subsubsection{4 von Hippel-Lindau Syndrome}

von Hippel-Lindau (VHL) syndrome (OMIM: 193300) is an autosomal dominant disorder caused by mutations in the $V H L$ gene on chromosome 3p25. Individuals with $V H L$ mutations are prone to developing retinal and central nervous system hemangioblastomas (HAB), endolymphatic sac tumours, clear cell renal cell carcinomas (RCC), pancreatic tumours, and PPGL (Kaelin, 2007; Maher, 2004). VHL syndrome is divided into two clinical subtypes; type 1 presents with RCC and HAB but not PPGL, type 2 is further divided with type $2 \mathrm{~A}$ characterised by HAB and PPGL but not RCC, type 2B characterised by HAB, RCC, and PPGL, and type 2C which presents with PPGL only. Mutations in $V H L$ account for around 13\% of familial and 9\% of sporadic PPGL (Dahia, 2014).

The VHL protein, in concert with elongin C (TCEB1), elongin B (TCEB2), Cullin-2 (CUL2), and ringbox 1 ( $R B X 1)$, forms a complex possessing ubiquitin ligase functionality marking substrate proteins for degradation (Kibel et al., 1995; Stebbins et al., 1999). The most notable substrates of the VHL complex are the alpha subunits of HIF. Under normoxic conditions, HIF1 $\alpha$ subunits are hydroxylated by prolyl hydroxylase (PHD) proteins through an oxygen-dependant mechanism. Subsequently, the hydroxylated HIF $1 \alpha$ is captured and ubiquitinated by the VHL complex marking it for degradation. Under hypoxic conditions, the activity of the PHD proteins is diminished leading to the stabilisation of HIF. The stabilised HIF promotes transcription of genes involved in a wide variety of cellular processes including metabolism, cell cycle progression, and angiogenesis (Hon et al., 2002; Kaelin, 2007; Keith et al., 2012; Maxwell et al., 1999).

In addition to regulation of HIF degradation, VHL also plays an important role in maintaining extracellular matrix (ECM) integrity. The participation in each role is determined by post-translational modification of VHL involving the addition of the ubiquitin-like molecule NEDD8. The neddylation of VHL creates steric hindrance preventing VHL from participating in the ubiquitin ligase complex (Russell \& Ohh, 2008). Immunoprecipitation studies have shown that VHL interacts with ECM components fibronectin (Ohh et al., 1998) and collagen IV alpha 2 (Kurban et al., 2008) and that loss of these interactions results in the disruption of ECM formation. While the exact mechanism by which VHL regulates ECM assembly is unclear, preliminary evidence suggests ECM dysregulation may be a result of inadequate activation of the small GTPase RhoA (Feijoo-Cuaresma et al., 2008). Interestingly, mutations that cause VHL syndrome types 1,2A, and 2B have been shown to be deficient in both HIF regulation and fibronectin binding, whereas mutations leading to type $2 \mathrm{C}$ disease are capable of ubiquitinating HIF but unable able to bind fibronectin (Clifford et al., 2001). This suggests multiple cell-specific roles for VHL in oncogenesis. 


\subsubsection{SDHA/B/C/D and SDHAF2}

Succinate dehydrogenase (SDH) is a four subunit protein complex bound to the inner mitochondrial membrane that is integrally involved in cellular metabolism. During the tricarboxylic acid (TCA) cycle, $\mathrm{SDH}$ is responsible for oxidising succinate to fumarate by transferring two hydrogen ions to flavin adenine dinucleotide (FAD). The electrons transferred to FAD by SDH, also known as complex II, are shuttled into the electron transport chain for use in oxidative phosphorylation (Lehninger et al., 2000).

Mutations in any of the SDH subunits leads to a failure in the conversion of succinate to fumarate (King et al., 2006). The resulting accumulation of excess succinate disrupts the function of a diverse class of enzymes known as the $\alpha$-ketoglutarate $(\alpha-\mathrm{KG})$ dependent dioxygenases, which includes the hypoxiainducible factor prolyl hydroxylases (PHD), jumonji histone demethylases (JMJD) and TET DNA hydroxylases.

PHDs are involved in post-translational modification where they catalyse the hydoxylation of prolyl residues by transferring one oxygen atom from molecular oxygen to $\alpha-\mathrm{KG}$ and the other to a prolyl residue, producing carbon dioxide, succinate, and hydroxy-prolyl. As mentioned previously, the hydroxylation of two prolyl residues is the first step in the degradation of HIF1 $\alpha$. In a normal cell under standard oxygen conditions the progression of this reaction is constant, it is only when the level of oxygen is reduced that this reaction can no longer proceed, the result of which is that HIF $1 \alpha$ is allowed to accumulate triggering the hypoxia response. The accumulation of succinate mimics the low oxygen condition by inhibiting the function of PHDs through both competitive (with $\alpha-K G$ ) inhibition and product inhibition, leading to a perpetual state of, what has been termed, pseudo-hypoxia (King et al., 2006).

The jumonji proteins are a class of JmjC domain containing histone demethylases (JHDM) that depend on molecular oxygen and $\alpha-K G$ for their activity. Histones, which form the structural component of nucleosomes around which DNA is wrapped to form chromatin, are subject to post-translation modification such as acetylation and methylation. These epigenetic modifications govern the transcriptional activity of genes by both physically altering the topology of DNA and providing signals to transcriptional machinery. Disruptions to normal methylation patterns have been implicated in a variety of cancers (Varier \& Timmers, 2011). Knockdown models examining the effect of reduced SDH in cultured cells have demonstrated an increase in the levels of histone methylation (H3K27me3 and H3K36me2), furthermore, the phenotype was shown to be reversible by over-expression of the functionally active C-terminal region of Jmjd3, a H3K27me3-specific histone demethylase (Cervera et al., 
2009). In yeast, phenotypic comparison of a strain harbouring a deletion of the JHDM orthologue, Jhd1, with an Sdh2 knockout strain showed a similar increase in occupancy of H3K36me2 relative to the wildtype strain. Wild-type strains could be made to partially mimic the knockout phenotype by treatment with exogenous succinate (Smith et al., 2007).

Similarly to histone methylation, methylation of DNA bases provides an important mechanism of epigenetic regulation. DNA methylation involves the covalent addition of a methyl group to a cytosine base, a process that occurs almost exclusively to bases in a $\mathrm{CpG}$ dinucleotide context. High densities of $\mathrm{CpG}$ dinucleotides, known as $\mathrm{CpG}$ islands, are often found in the promoter regions of genes. In this context, their state of methylation confers transcriptional repression (methylated) or expression (unmethylated). In recent times aberrant DNA methylation has been increasingly recognised as a mechanism for the silencing of tumour suppressor genes such as $M L H 1, V H L, C D K N 2 A$, and $B R C A 1$ (Baylin, 2005). The TET DNA hydroxylases are a family of enzymes that catalyse the conversion of 5methylcytosine $(5 \mathrm{mC})$ into 5-hydroxymethylcytosine $(5 \mathrm{hmC})$, the first step in cytosine demethylation (Guo et al., 2011). Over-expression of the catalytic domain of TET1 in cultured cells produces a detectable increase in the level of $5 \mathrm{hmC}$ but when combined with the knockdown of SDHA or SDHB, the levels of $5 \mathrm{hmC}$ are reduced by $83 \%$ and $69 \%$ respectively (Xiao et al., 2012). Methylome analysis of a large PPGL cohort found that samples separated into three distinct classes based on their methylation phenotype, designated M1, M2, and M3. Class M1, consisting primarily of SDH-mutant tumours, exhibited a hypermethylator phenotype characterised by high methylation levels both within and outside of CpG islands. Classes M2, predominantly VHL tumours, and M3, NF1/RET type tumours, lacked the hypermethylator phenotype and exhibited a hypomethylation phenotype outside CpG islands (Letouze et al., 2013). Combined, these findings suggest a strong role for oncometabolites resulting from SDH mutations in methylome dysregulation.

The clinical phenotype of SDH mutations is variable depending on the defective subunit. Loss of SDHD function gives rise to paraganglioma syndrome 1 (PGL1) (MIM:168000), which is characterised by the development of multiple PGL, particularly in the head and neck region, and less frequently PCC, with a low rate of malignancy (Benn et al., 2015; Dahia, 2014; Opocher \& Schiavi, 2011). PGL2 (MIM: 613019) is associated with mutations in the SDHAF2 gene, required for the flavination of SDH, and has similar clinical phenotype to PGL1 with the exception of adrenal PCC, which have not been reported (Hao et al., 2009). PGL3 (MIM:605373), which is caused by mutations in SDHC, leads to the development of PGL and carotid body tumours but not adrenal PCC, while development of concurrent tumours or metastatic disease are rare (Schiavi et al., 2005). Inactivating mutations in SDHB are 
associated with PGL4, an autosomal dominant disease marked by the development of head and neck paragangliomas, PCC, and PGL, with a high rate of malignancy (Waldmann et al., 2009). Recently, mutations in SDHA have been described in both PCC and PGL (Burnichon et al., 2010; Korpershoek et al., 2011).

In 2002, a novel syndrome, known as the Carney-Stratakis syndrome, was described as imbuing a propensity to develop both PGL and gastrointestinal stromal tumours (GIST). In 2008, a screen of 11 GIST patients lacking a typical KIT or PDGFRA mutation found mutations in $S D H B, C$, and $D$ in 5, 2 and 1 patients respectively (Pasini et al., 2008). A finding supported by further research showing that GISTs with wild-type KIT and PDGFRA have either complete loss, or a substantial reduction in, SDHB protein expression (Janeway et al., 2011). However, the exact relationship between SDH and Carney-Stratakis syndrome remains to be elucidated.

\subsubsection{Non-syndromic PPGL}

\subsubsection{Germline Mutations in Non-syndromic PPGL}

\subsection{MAX}

MAX, or MYC-associated factor $\mathrm{X}$, is a member of a highly evolutionarily conserved transcription modulation network. The MYC/MAX/MXD triad forms the core of a complex network of DNA interacting proteins, modulating a diverse and sometimes antagonistic set of cellular processes such as proliferation, growth-arrest, and differentiation (Diolaiti et al., 2014). The MAX/MYC heterodimer is able to bind to the E-box (Enhancer Box) sequence found in the promoter region of many genes, where upon doing so, it promotes gene transcription. Conversely, the MAX/MXD heterodimer, which also binds E-box sequences, prevents transcriptional activation upon binding. Research suggests that MYC and MXD compete for MAX binding and that the ultimate outcome of the regulatory network results from the relative abundance of each partner (Ayer et al., 1993).

In 2011, exome sequencing of three PCC with similar transcriptional profiles uncovered germline mutations in the $M A X$ gene (Comino-Mendez et al., 2011). The tumours exhibited coordinate loss of the wild-type allele as either chromosomal loss or copy neutral loss of heterozygosity. A follow-up study sequencing the $M A X$ gene in 1,694 PPGL patients without a known mutation found $M A X$ to be altered in $1.12 \%$ of cases (Burnichon, Cascon, et al., 2012). 


\subsection{TMEM127}

In 2010, genomic analysis of a family with multiple individuals affected by PCC revealed a mutation in the transmembrane protein TMEM127 (Qin et al., 2010). Although the exact function of TMEM127 is unknown, the similar transcription profile of TMEM127 mutant tumours and RET/NF1 tumours suggested the oncogenic mechanism might be through the RAS signalling pathway. However, initial TMEM127 knockdown experiments showed no additional RAS activity (Qin et al., 2010). As loss of $N F 1$ has been shown to result in dysregulation of mTOR signalling, Qin and colleagues examined phosphorylation of 4EBP1 and S6K, markers of mTORC1 activation, and AKT, a marker of mTORC2 activation. They found that knockdown of TMEM127 resulted in increased levels of 4EBP1 and S6K but not AKT phosphorylation. Conversely, over expression of TMEM127 showed reduced mTORC1 signalling (Qin et al., 2010). These results suggest that TMEM127 may have a role in regulation of mTORC1 but not mTORC2 signalling. Examination of the cellular localisation of wild-type TMEM127 suggests it is typically either integrated into the plasma membrane of the cell or that of membrane bound organelles. In contrast, mutant TMEM127 demonstrates a diffuse cytoplasmic distribution (Yao, Schiavi, et al., 2010). Wild-type TMEM127 has also been shown to colocalise with activated mTORC1, suggesting that it may have a role in trafficking or regulating exposure of mTORC1 to other regulatory factors (Jiang \& Dahia, 2011). Large cohort studies estimate the frequency of TMEM127 mutations in PPGL to be between 0.9 and 2\% (Abermil et al., 2012; Yao, Schiavi, et al., 2010).

\subsection{KIF1B $\beta$}

KIF1B codes for two isoforms of a motor protein, KIF1B $\alpha$ and KIF1B $\beta$, which are implicated in anterograde transport of mitochondria and synaptic vesicle precursors. The beta isoform has been shown to have a proapoptotic function (Munirajan et al., 2008). During development, programmed cell death is integral to formation of correct neural connections and its occurrence is tightly controlled by the levels of neurotrophic factors. One such neurotrophic factor, nerve growth factor (NGF) binds to the cell surface receptor TrkA triggering a blockade of the apoptotic process (Dekkers et al., 2013). KIF1B $\beta$ has been implicated in this process as a downstream effector of TrkA signalling. Ectopic expression of KIF1B $\beta$ has been shown to increase caspase activity and induce apoptosis. This ability is thought to be independent of its function as a motor protein, as constructs lacking the motor domain retain the proapoptotic functionality. Recently, KIF1B $\beta$ has been shown to be responsible for the localisation of DHX9 to the nucleus where it promotes the transcription of proapoptotic factor XAF1 (Chen et al., 2014). These data suggest that mutations in KIF1B may undermine programmed cell death in cells of neural origin, priming them for oncogenic transformation. 
At present there are only a small number of reports of KIF1B mutations in PPGL, suggesting that the overall prevalence is likely to be very low (Schlisio et al., 2008; Yeh et al., 2008).

\subsection{EGLN1 (PHD2)}

EGLN1 is a member of the prolyl-hydroxylase domain (PHD) family of proteins. As discussed previously, these proteins hydroxylate HIF- $\alpha$ subunits in an oxygen-dependent manner marking them for destruction. At present only a single case of PGL has been linked to a mutation in EGLN1 (Ladroue et al., 2008). Studies looking for mutations in the remaining PHDs, EGLN2 and EGLN3, were unable to find evidence of mutations in these genes (Astuti et al., 2011).

\subsection{IDH1}

Isocitrate dehydrogenase catalyses the oxidative decarboxylation of isocitrate to produce $\alpha-\mathrm{KG}$. As such, failure of IDH can result in inadequate levels of $\alpha-K G$ leading to a disruption of $\alpha-K G$ dependant dioxygenases through a similar mechanism to succinate dehydrogenase failure. In light of this mechanistic similarity and the identification of $I D H 1$ mutations in neural type tumours (Bleeker et al., 2009), two studies examined the prevalence of $I D H 1$ mutations in PPGL. The first study examined 365 PCC, PGL, and head and neck paragangliomas, finding no mutations in PCC or PGL, but finding a single p.Arg132Cys mutation in a sporadic carotid paraganglioma (Gaal et al., 2010). The second study sequenced codons 132 of IDH1 and 172 of IDH2 in 104 PCC and found no mutations (Yao, Barontini, et al., 2010). These findings suggest that although $I D H 1$ mutations may have a similar biochemical phenotype to SDH mutations, they do not play a significant role in PPGL tumourigenesis.

\subsection{FH}

During the TCA cycle, fumarate hydratase (FH) catalyses the conversion of fumarate to malate through the addition of a water molecule. Individuals with a germline mutation in $F H$ are predisposed to hereditary leiomyomatosis and renal cell cancer (Lehtonen, 2011). However, the association with PPGL was not described until recently. In 2013, a survey of 598 patients without mutations in the known PPGL susceptibility genes uncovered five pathogenic germline $F H$ mutations (Castro-Vega et al., 2013), while a more recent study of 72 patients found two novel mutations (Clark et al., 2014). Examination of the levels of 5-hmC in $\mathrm{FH}$ tumours by IHC demonstrated a similar pattern of methylation to that observed in SDHx mutant tumours. This suggests a common mechanism of dioxygenase inhibition by metabolite accumulation. Overall $\mathrm{FH}$ mutations are likely to account for a small proportion of PPGL, but since three of the seven patients described so far exhibited metastatic disease, suggesting that, like $S D H x$ tumour, $F H$-driven disease, like $S D H x$ tumours, may have a higher malignant potential. 


\subsection{MDH2}

Malate dehydrogenase $2(\mathrm{MDH})$ is the most recent addition to the growing list of TCA cycle genes which, when mutated, confer susceptibility to PPGL development. A study in which whole exome sequencing was performed on a 55-year-old male with multiple malignant paragangliomas identified a donor splice site mutation on exon 4 of $M D H 2$ (Cascon et al., 2015). The authors demonstrated that the mutation resulted in incorporation of an additional 20 amino acids followed by a premature stop codon. The mutant transcript was demonstrated to undergo nonsense-mediated decay. The role of $M D H 2$ in the TCA is to catalyse the oxidation of malate to oxaloacetate; surprisingly the authors were unable to detect accumulation of the substrate as has been seen with the dysfunction of other TCA enzymes. However, an increased fumarate to succinate ratio was observed, suggesting a potential accumulation of fumarate similar to that of $\mathrm{FH}$ mutant tumours. Furthermore, $\mathrm{MDH} 2$ mutant tumours were shown to exhibit the hypermethylator phenotype seen in $S H D x$ and $F H$ tumours. Pathogenicity was ultimately confirmed by demonstrating segregation of the mutation with disease in related individuals. At present there is only this single report of $\mathrm{MDH} 2$ mutations in PPGL and, as such, the prevalence remains unknown.

\subsubsection{2 de Novo and Sporadic mutations in Non-syndromic PPGL}

\subsection{EPAS1}

The EPAS1 gene encodes the protein HIF2 $\alpha$, a member of the hypoxia inducible factor group of transcription factors. These transcription factors are master regulators of the cellular response to oxygen availability. As touched on previously, under normoxic conditions the HIF $\alpha$ subunits (HIF1 $\alpha$, HIF2 $\alpha$, and $\mathrm{HIF} 3 \alpha$ ) are rapidly degraded. If cellular oxygen levels are reduced, the HIF $\alpha$ subunits escape destruction, translocate to the nucleus, and bind to the HIF1 $\beta$ subunit (also known as ARNT). The heterodimer then recruits the transcription co-factors CREB-binding protein and p300, and binds to HIF-responsive elements (HRE), promoting the transcription of a vast array of genes (Maxwell et al., 2001).

Despite the central role that HIF signalling plays in many cancers, mutations in the HIF2 $\alpha$ subunit had only previously been described in relation to polycythemia, a disorder in which the proportion of blood volume occupied by red cells is increased (Bento et al., 2014). Recently, however, a case report was published describing two patients with congenital polycythemia and young onset multiple PGLs (Zhuang et al., 2012). Interestingly the mutations were not found in constitutional DNA taken from each of the 
patients suggesting that the mutations were likely de-novo events in a common precursor cell during early embryogenesis. A subsequent study found EPAS1 mutations in three patients with concomitant PGL and congenital polycythemia, as well as an additional four sporadic cases in the absence of polycythemia (Comino-Mendez et al., 2013). All mutations were found to affect residues at, or in close proximity to, the primary hydroxylation site, resulting in increased stability without affecting DNA binding efficiency. A more recent study sequencing 167 sporadic PPGL found four (2.3\%) HIF2 $\alpha$ mutations, whilst screening of HIF1 $\alpha$ uncovered no mutations (Toledo et al., 2013). Combined, the recent literature suggests that mutation in HIF $2 \alpha$ account for between 2 and $5 \%$ of sporadic PPGL.

\subsection{HRAS}

HRAS is a member of the p21 RAS GTPase family of signalling effector proteins which form a major convergence point for cellular signalling. Typically, activation of a receptor tyrosine kinase by ligand binding leads, through various intermediaries, to activation of guanadine exchange factors (GEFs). These GEFs assist with the conversion of inactive GDP-bound RAS to the active GTP-bound form. Once activated, RAS further activates a number of downstream effectors such as RAF, PI3K, and PLC. Through these pathways, RAS plays a significant role in a diverse array of cellular processes including cell growth, cell-cycle progression, apoptosis, and cytoskeletal organisation. Since the oncogenic potential of the RAS genes was first discovered in the 1970s they have been shown to be involved in the development of $30 \%$ of all cancers (Saxena et al., 2008). HRAS specifically has been found to be mutated in cancers of the urinary tract $(11 \%)$, salivary gland (15\%), prostate $(6 \%)$, and cervix (9\%) (PylayevaGupta et al., 2011). HRAS mutations in PPGL were first described in 1992, where a small cohort study of 19 tumours revealed a single case with a mutation in codon thirteen (Yoshimoto et al., 1992). More recently, two studies examining the contribution of $H R A S$ to PPGL discovered mutations in four of 58 (6.9\%) and fourteen of 271 (5.2\%) PPGLs (Crona et al., 2013; Oudijk et al., 2014).

\subsection{BRAF}

BRAF, an effector kinase immediately downstream of RAS, is the most recent component of the mitogenactivated protein kinase pathway (MAPK) to be linked to PPGL. Mutations in BRAF are common in a wide range of cancers including melanoma ( $\sim 60 \%)$, colorectal (5-20\%), and low-grade serous ovarian cancer (33-50\%) (Burotto et al., 2014; Davies et al., 2002). Luchetti et al. applied massively parallel sequencing to examine recurrently mutated sites in 50 known cancer genes across 85 PPGL samples (Luchetti et al., 2015). This approach detected a single sample with a somatic mutation (c.1799T $>$ A) in $B R A F$ resulting in the canonical p.V600E amino acid change. At present this the only report of $B R A F$ mutations in PPGL and, as such, the overall contribution to the incidence of PPGL is unknown. 


\subsubsection{Pathways in PPGL Oncogenesis}

In 2005, Dahia et. al. performed unsupervised clustering of gene-expression analysis on 76 PPGL. They observed that the tumours formed 2 major clusters; cluster one, comprised mainly of SDHx and $V H L$ mutant tumours, and cluster two containing tumours with RET and NF1 mutations, as well as a large number of sporadic tumours of unknown genotype (Dahia et al., 2005). This picture was further refined by a 2011 study by Nelly Burnichon and colleagues who performed unsupervised clustering of 69 hereditary and 119 sporadic PPGL (Burnichon et al., 2011). The analysis revealed that cluster 1 further segregates into 2 subgroups, a group designated 1A containing all of the SDHx related tumours and two sporadic tumours, and group $1 \mathrm{~B}$ containing all except one of the $V H L$-related tumours and 16 sporadic tumours. Cluster two segregates into subgroup 2A representing all of the NF1 and TMEM127 related tumours, and all except one of the RET related tumours. Cluster 2B was composed entirely of sporadic tumours and group 2C contained the remaining VHL and RET tumours along with 12 sporadic tumours. Subsequent sequence analysis identified $V H L$ mutations in 11 of the 16 Cluster 1B sporadic tumours, and RET mutations in 6 of the 60 sporadic group 2A tumours. More recently, mutations in FH and EPAS1 have been identified in apparently sporadic cluster one tumours (Castro-Vega et al., 2013; Zhuang et al., 2012), and $M A X$ mutations have been discovered in familial and sporadic cluster two tumours (CominoMendez et al., 2011).

\subsubsection{Pseudo-Hypoxia}

Cluster 1 tumours have been termed pseudo-hypoxic based on their shared dysregulation of HIF signalling. Through the mechanisms described previously, mutations in $S D H x, V H L, F H, M D H 2$, and EGLN1 result in the stabilisation of HIF $\alpha$ subunits and subsequent transcription of HIF target genes. Genome-wide immunoprecipitation studies have found between 377 and 546 HIF1 $\alpha$ and around 150 HIF2 $\alpha$ binding sites (Mole et al., 2009; Xia et al., 2009). A portion of these sites lack the canonical HRE sequence and may represent HIF $\alpha$ in complex with other DNA binding proteins. At present, HIF has been shown to directly regulate the expression of over 100 genes (Liu, Shen, et al., 2012), and likely affects the expression of others through regulation of microRNAs (Kulshreshtha et al., 2007), interaction with other signalling pathways (Dang et al., 2008; Koshiji et al., 2004), and regulation of transcription factors (Peng et al., 2008). 


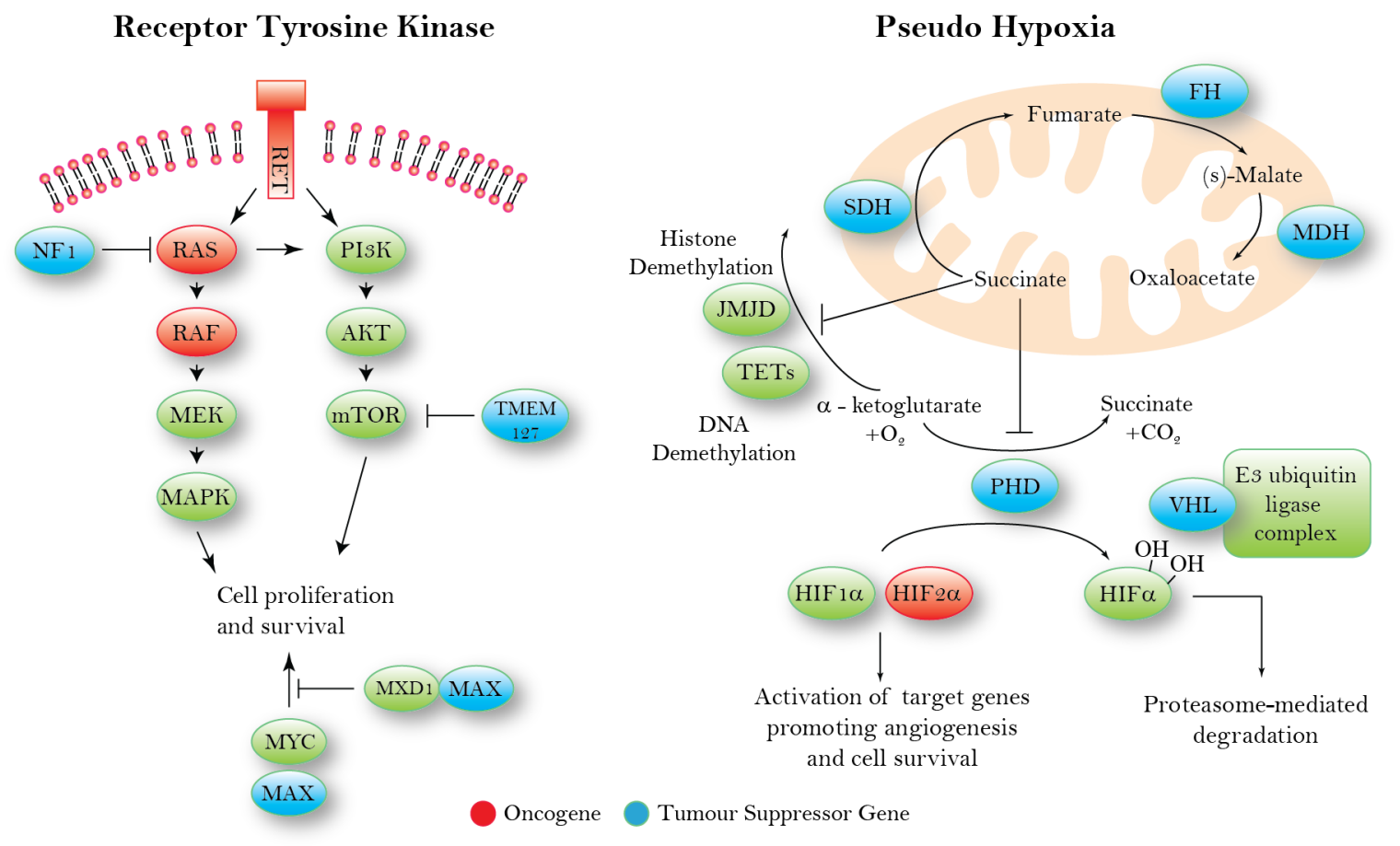

Figure 1.1 Pathways in PPGL

In receptor tyrosine kinase type PPGL, activating mutations in the receptor tyrosine kinase $R E T$ and the effector proteins RAS and $R A F$, as well as inactivating mutations in NF1, a negative regulator of RAS, and TMEM127, a modulator of $\mathrm{mTOR}$, lead to excess activation of the mitogen activated protein kinase (MAPK) and mTOR signalling pathway. In pseudo-hypoxia type PPGL, inactivating mutations in several tricarboxylic acid cycle enzymes lead to substrate accumulation, resulting in inhibition of prolyl hydroxylases (PHD) and stabilisation of HIF. Similarly mutations in VHL, which forms part of a ubiquitination complex responsible for marking HIF for proteasomal degradation, result in stabilisation of HIF under normoxic conditions.

The HIF target genes traverse a wide range of cellular processes including angiogenesis, energy metabolism, cell differentiation and apoptosis, which are commandeered during oncogenesis. One of the first described targets of HIF was vascular endothelial growth factor (VEGF), a signalling molecule that promotes the development of neovasculature, a process integral to tumour growth and survival (Ellis \& Hicklin, 2008). The effects of activated HIF and VEGF signalling are reflected in the high degree of vascularisation in PPGL, particularly those belonging to cluster 1. Additional HIF targets include enzymes involved in glycolytic metabolism such as the glucose transporter GLUT1, hexokinase 2 (HK2), and pyruvate kinase 1 (PDK1)(Chi et al., 2006). The upregulation of these genes represents a shift away from oxidative phosphorylation towards glycolysis as the primary metabolic pathway; known as the Warburg effect, this shift in energy metabolism is a feature of many types of cancer (Chi et al., 2006). Interestingly, jumonji histone demethylases have also been shown to be a target of HIF transcription (Xia 
et al., 2009). As these enzymes are oxygen-dependant, their function is impaired under hypoxic conditions. The upregulation by HIF likely represents an attempt by the cell to compensate for low oxygen conditions and maintain the methylation steady state. However, it seems in $F H / S D H x$-driven pseudo-hypoxia increased expression is insufficient to overcome product inhibition (Letouze et al., 2013). Adding to the complexity of HIF signalling is the finding that HIF1 $\alpha$ and HIF2 $\alpha$ have both shared and mutually exclusive gene targets (Keith et al., 2012). Working from the assumption that HIF overexpression represented an oncogenic facilitator in non-small cell lung cancer, Mazumdar et al. performed knockdown experiments on HIF1 $\alpha$ and HIF2 $\alpha$ (Mazumdar et al., 2010). Rather than observing a reduction in cellular proliferation, knockdown of HIF1 $\alpha$ resulted in no change while knockdown of HIF $2 \alpha$ resulted in increased proliferation. The authors concluded that diminished expression of the HIF $2 \alpha$ target $S C G B 3 a 1$, a candidate tumour suppressor gene, was responsible. This apparent paradox highlights the immense complexity in understanding the outcome of dysregulated HIF signalling. As such, discerning exactly which targets of HIF are important in the initiation and progression of PPGL represents a significant challenge.

\subsubsection{MAPK Signalling}

Cluster 2 tumours share a dysregulation of mitogen-activated protein kinase (MAPK) signalling. RTKs are proteins that consist of an extracellular receptor domain and intracellular kinase domain. Typically, a ligand binding to the extracellular domain triggers dimerisation facilitating autophosphorylation of the intracellular domains. The phosphorylated residues then act as docking sites for downstream signalling effector molecules.

As discussed previously, the receptor tyrosine kinase RET follows this paradigm. Phosphorylation studies have shown that fourteen of the eighteen tyrosine residues of the RET protein can become phosphorylated (Kawamoto et al., 2004; Knowles et al., 2006). The particular residues phosphorylated determine which downstream effector molecules bind and thus which signalling pathway is activated (Murakumo et al., 2006). Phosphorylation of reside Y905 allows binding of SRC, which then activates the PI3K signalling pathway promoting cell survival (Melillo et al., 1999). Similarly, phosphorylated Y1062 (pY1062) can lead to PI3K activation through SHC binding and subsequent activation of GRB2 and GAB1/2 (Arighi et al., 1997; Murakumo et al., 2006). Interaction of FRS2 with pY1062 also activates GRB2, in this instance GRB2 complexes with SOS to promote the removal of GDP from members of the RAS family, allowing the subsequent activation RAS through the binding of GTP (Melillo et al., 2001). Other signalling pathways activated by RET include c-JUN NH2-terminal kinase (JNK), phospholipase C $\gamma$, and 
signal transducer and activator of transcription 3 (STAT3) (Murakumo et al., 2006; Plaza Menacho et al., 2005).

Mutations in the RET gene either lead to persistent dimerisation in the absence of ligand or constitutive activation of the protein kinase domain (Asai et al., 1995; Borrello et al., 1995; Santoro et al., 1995). Transfection of NIH 3T3 cells with mutant RET is sufficient for transformation, however, additionally mutating Y1062 was shown to significantly impair the transforming activity (Asai et al., 1996). It is not yet known how many of the signalling pathways activated by RET are involved in PPGL oncogenesis, however, the RAS signalling cascade is certainly a major contributor. This is evident in the contribution of both deactivating mutations in $N F 1$, a negative regulator of RAS, and activating mutations in $H R A S$ itself to PPGL development.

TMEM127-driven PPGLs also fall into cluster two, although the link with MAPK signalling is less clear. TMEM127 has been implicated in the early-to-late endosomal transition by regulating the function of RAB5 (Qin, Deng, et al., 2014), failure of which is thought to result in enhanced activity of lysosomalbound mTOR ( $\mathrm{Li}$ et al., 2010). Interestingly, the mTOR signalling pathway is in part regulated by RAS signalling through the activation of ERK. Phosphorylation of TSC2 by ERK has been shown to disrupt the TSC1-TSC2 complex impairing negative regulation of mTOR (Ma et al., 2005). PI3K regulated phosphorylation of TSC2 by AKT and subsequent mTOR activation may also contribute to oncogenic RET signalling independently of RAS (Rapa et al., 2011). Additional research is required to further elucidate the mechanisms of TMEM127 oncogenesis and the role of mTOR signalling in PPGL development.

\subsubsection{MAX/MXD/MYC}

As mentioned previously, $M A X$-driven PPGL have also been shown to belong to cluster 2. However, these tumours tend to form a distinct subcluster away from the RET/RAS/NF1 type tumours, potentially suggesting a related but distinct mechanism of oncogenesis. Further support of this distinction is seen in the expression of the gene phenylethanolamine N-methyltransferase (PNMT), which encodes the enzyme responsible for the conversion of norepinephrine to epinephrine in the final step of catecholamine biosynthesis. PNMT is thought to provide a surrogate marker for the differentiation state of chromaffin cells. Cluster 1 tumours exhibit low or absent expression of PNMT, indicative of a less differentiated state, whereas non-MAX cluster 2 tumours express higher levels. $M A X$-related tumours have an intermediate level of PNMT expression suggesting a partially differentiated phenotype (Qin, de Cubas, et al., 2014). As discussed previously, the MAX/MXD/MYC triad forms an intricate regulatory network, 
dysregulation of which, often through MYC amplification, has been found in many cancers (Calcagno et al., 2008; Chen \& Olopade, 2008; Sewastianik et al., 2014), including the closely related neuroblastoma (Westermark et al., 2011). Experiments using the rat PCC line PC12, which lacks a functional MAX gene, demonstrated that reintroducing $M A X$ expression affected the expression of over 3000 genes including 89 involved in cell proliferation and differentiation (Qin, de Cubas, et al., 2014). The authors also demonstrated that Pnmt expression in MPC cells is modulated by MAX, with siRNA abrogation of Max expression resulting in a reduction in PNMT activity. The large scope of the gene network regulated by the MAX/MXD/MYC triad presents a significant obstacle to understanding the specific genes involved in MAX-driven oncogenesis.

Additionally, a link between MAX/MYC and HIF signalling may come from the observation that HIF $\alpha$ subunits are capable of modulating MAX/MYC activity in a number of ways. Firstly, expression of MXI1, which regulates MYC activity by binding competitively with MAX, has been shown to be upregulated by increased HIF signalling (Corn et al., 2005). Secondly, HIF1 $\alpha$ has been shown to attenuate the cellular level of MYC through a proteasome-dependent mechanism (Zhang et al., 2007). Thirdly, HIF $1 \alpha$ has been shown to displace MYC from SP1 binding leading to repression of MYC target genes (Koshiji et al., 2005). Finally, HIF1 $\alpha$ can bind directly to MAX sequestrating it from MYC (Dang et al., 2008). In contrast to HIF $1 \alpha$, HIF $2 \alpha$ appears to enhance MYC activity by binding to and stabilising the MYC/MAX complex (Gordan et al., 2007). To what extent the MYC target genes that are dysregulated in MAX mutant tumours are also involved in the oncogenesis of pseudo-hypoxia tumours is yet to be explored.

\subsubsection{Neuronal Apoptosis and Developmental Culling}

In 2005, Lee et al. proposed that PPGL was the result of a failure of apoptosis during developmental culling of neural precursor cells. As previously discussed, developing neural cells are dependent on NGF to prevent activation of the apoptotic machinery. In the event of NGF withdrawal, discontinuation of TrkA signalling results in programmed cell death via a c-Jun and EgIN3 dependant mechanism. Lee et al. demonstrated that mutations in VHL increased the levels of JunB resulting in an attenuation of c-Jun activation preventing apoptosis (Lee et al., 2005). The authors also postulated that succinate accumulation resulting from a mutation in the SDH subunits would prevent EgIN3, acting downstream of c-Jun, from triggering apoptosis. More recently, the discovery that KIF1B $\beta$ acts as a downstream effector in the NGF/TrkA signalling pathway by promoting the transcription of proapoptotic factors has added support to the theory. However, it is not clear what role the remaining driver genes play in this model, or how somatic mutations arising after developmental culling would contribute to PPGL tumourigenesis. 


\subsubsection{Auxiliary Genomic Changes}

The gene mutations discussed previously can be considered primary or initiating driver events. It is now estimated that a typical tumour has between two and eight driver mutations (Vogelstein et al., 2013). In addition to single nucleotide substitutions and small-scale insertions and deletions, tumours can undergo large-scale genomic alteration in the form of chromosomal gains, losses, and structural rearrangements.

\subsubsection{Copy-number Alterations}

In 1971, after observing that the incidence of bilateral disease in patients with inherited retinoblastoma was greater than in sporadic cases, Alfred Knudson proposed that two events were required for retinoblastoma formation (Knudson, 1971). This could either be a germline mutation followed by a somatic event in the case of inherited disease, or two somatic events in the case of sporadic disease. While Knudson postulated that the second event would be in a gene site distinct from the first, it is now known that for inherited retinoblastoma the second in event Knudson's "two-hit" hypothesis is the loss or mutation of the second copy of the $R B 1$ gene. This and other observations has led to the broader classification of genes as either tumour suppressors, for which oncogenesis requires loss of the gene function, or oncogenes, for which oncogenesis is reliant on enhanced or unregulated function of the gene.

The genes currently known to contribute to PPGL are, with the exception of RET, HRAS, BRAF, and EPAS1, predominantly tumour suppressor genes, as such, PPGL tumourigenesis generally adheres to Knudson's two-hit model. While loss of the wild-type allele can occur through a somatic mutation, chromosomal loss provides the predominant mechanism and has been reported for VHL (Crona et al., 2014; Zeiger et al., 1995), SDHA/B/C/D (Beristain et al., 2013; Burnichon et al., 2010), TMEM127 (Abermil et al., 2012), NF1(Burnichon, Buffet, et al., 2012), MAX (Burnichon, Cascon, et al., 2012), and FH (Castro-Vega et al., 2013). Conversely, amplification of oncogenic mutations through gain of chromosome 10 is a feature of RET mutant tumours, resulting in either trisomy or copy neutral loss of heterozygosity with retention of the mutant allele (Huang et al., 2000).

In addition to alterations to the chromosomes harbouring a driver mutation, frequent chromosomal losses involving chromosomes $1 \mathrm{p}, 3,6,11,17,21$, and 22, and less commonly gains involving chromosomes 1q, 12, and 19 have been reported (Dannenberg et al., 2000; Edstrom et al., 2000; Jarbo et al., 2006; Petri et al., 2008; Sandgren, Diaz de Stahl, et al., 2010; van Nederveen et al., 2009). A more recent integrated 
analysis demonstrated a strong correlation between the primary driver event and copy-number alterations (Castro-Vega, Letouze, et al., 2015). Cluster 1A, consisting mostly of SDHB tumours, were defined largely by the loss of chromosome $1 \mathrm{p}$, representing loss of the wild-type copy of $S D H B$, with a small portion experiencing loss of 3p/q and 11p, and gains of 1q. The $V H L$ tumours (Cluster 1B) displayed almost ubiquitous loss of chromosome $3 \mathrm{p}$, again representing the loss of the wild-type allele, with most demonstrating loss of chromosome $3 \mathrm{q}$ and $11 \mathrm{p}$. Interestingly, Cluster 2A tumours (RET/NF1/TMEM127) also showed almost universal loss of chromosome $1 \mathrm{p}$ and $3 \mathrm{q}$, neither of which harbour the relevant drivers, and a portion demonstrated loss of $17 \mathrm{p} / \mathrm{q}, 21$, and 22 . These findings suggest that in order for tumour progression via a given driver, there are obligate complementary regions that must first be disrupted. Attempts to discern the critical genes in these regions have been met with limited success due to the fact that these events frequently involve large segmental changes, usually an entire chromosome or chromosome arm, suggesting that it is not a single gene or locus involved but rather a collection of genes spread across the regions.

There is currently very little knowledge surrounding the contribution of copy-number alterations to the malignant potential of PPGL. A single study comparing copy-number events observed that malignant tumours more frequently demonstrated chromosomal gains, particularly chromosome 12 and 19q (Sandgren, Diaz de Stahl, et al., 2010). The authors also noted loss of chromosome 11q was significantly associated with malignant tumours. Other studies attempting to look at specific loci have uncovered little, except for the observation that malignant tumours appear to have greater clonal heterogeneity with regard to chromosomal aberrations (Korpershoek et al., 2010; Petri et al., 2008). Most efforts to understand the molecular differences between benign and malignant PPGL are hampered by the paucity of malignant specimens. It is likely that in order to understand the contribution of chromosomal aberrations to malignant potential, a concerted multicentre effort will be required.

\subsubsection{Cooperating Gene Mutations}

The advent of massively parallel sequencing has ushered in a new age of genomic discovery, facilitating fine detail mapping of the genomic changes involved in over 100 cancer types to date (Cancer Genome Atlas Research et al., 2013; International Cancer Genome et al., 2010). This has led to the recognition that different cancer types can have very different mutational loads and signatures, examination of which can assist with understanding the mutational processes involved in oncogenesis (Alexandrov, Nik-Zainal, Wedge, Aparicio, et al., 2013; Vogelstein et al., 2013). For instance, C:G $>$ A:T transversions are common in smoking-related lung cancer, whilst $\mathrm{C}: \mathrm{G}>\mathrm{T}: \mathrm{A}$ transitions and CC:GG $>$ TT:AA double nucleotide substitutions are frequently seen in UV radiation-related skin cancers (Alexandrov, Nik-Zainal, 
Wedge, Campbell, et al., 2013; Krauthammer et al., 2012; Liu, Morrison, et al., 2012). Tumours associated with carcinogen exposure or those featuring mutations in DNA repair genes tend to have a mutational load orders of magnitude greater than others (Vogelstein et al., 2013). These genomic landscape studies have also furthered the "mountains and hills" paradigm, whereby a small number of genes are involved in a majority of cancers and a large number of genes are involved in only a small number of cancers. It is now estimated that there are at least 140 genes that, when altered, promote oncogenesis (Vogelstein et al., 2013).

More recently, high-sensitivity genomics techniques have begun to erode the concept of tumours as a homogeneous collection of cells with a static genotype. For instance, a study sampling nine spatially distinct regions of a clear cell renal cell carcinoma found that only around half the sequence variants were shared across all regions of the primary tumour, and as little as a third were shared between the primary tumour and the metastases (Gerlinger et al., 2012). Similarly, a study of multi-focal prostate cancer comparing regions of high and low Gleason scores with metastases found significant divergence (VanderWeele et al., 2014). In one case, only two of a combined 46 mutations were shared between high and low scoring regions. In a second case, 7 of a combined 79 mutations were shared between all regions, while 50 of the 63 mutations found in the metastasis were also present in the high scoring region. It is clear that in these cases the high and low scoring regions diverged at an early time point from a common ancestral clone, both continued to evolve and, in the second case, what became the high scoring region ultimately developed metastatic potential. These and other studies are only just beginning to uncover the complex evolutionary processes occurring within a developing tumour, understanding of which will be paramount in addressing acquired resistance to chemotherapeutic and targeted agents.

At the inception of this study very little was known about the genomic landscape of PPGL beyond the common copy-number alterations. It was clear that there was an information deficit regarding the auxiliary genes and pathways that may be recurrently altered or what mutational processes may be driving tumour evolution. It is only very recently that the first study describing a recurrently mutated cooperating gene, $A T R X$, was published (Fishbein et al., 2015). The $A T R X$ gene is a member of the SWI/SNF family of chromatin remodelling proteins that is involved in histone H3.3 loading in telomeric regions (Lewis et al., 2010), as well as chromosome alignment and meiotic spindle organization (De La Fuente et al., 2004). $A T R X$ has also been implicated in the Alternate Lengthening of Telomeres (ALT) mechanism identified in a range of cancer types (Lovejoy et al., 2012). Mutation or loss of ATRX had been previously described in other neural and neuroendocrine type tumours, suggesting it plays an important role in oncogenesis in these cell lineages (Abedalthagafi et al., 2013; Jiao et al., 2011). 
Following shortly after publication of the paper presented in Chapter Three of this dissertation, a second genomics study integrating exome sequencing with methylation, cytogenetic, transcriptomic, and miRNA profiling was published. The study found PPGL to have a low mutation rate of 0.3 mutations per megabase and that very few genes were recurrently altered. Tumour suppressor genes TP53 and CDKN2A were found to be mutated in $10 \%$ and $7 \%$ of cases respectively, while the oncogene $M E T$ was found to be mutated in $2.5 \%$ of cases. Other genes previously implicated in cancer that were found to be mutated in the study include CDH1, ARHGEF12, SLC45A3, SMO, CARD11, MLL2, PCM1, GNAS, FLT3 and FHIT. The copy-number alterations described are consistent with previous studies, comprising mainly large segmental losses involving chromosomes 1p, 3, 11, 17p, 21, and 22.

The authors performed consensus clustering, a method for discerning the most stable number of groups within a data set, on miRNA data from 172 PPGLs. They found seven distinct subclasses which largely correlated with the classes determined through unsupervised clustering of mRNA data. Interestingly, loss of expression of the maternally expressed $M E G 3$ and down regulation of miRNAs in the DLK1-MEG3 cluster at 14q32 was correlated with a subset of PPGL for which no known driver was found. Specific loss of the maternal copy of this region has previously been described in $M A X$-related PPGL, which exhibits uniparental disomy corresponding with paternal transmission of the mutation (Comino-Mendez et al., 2011). However, these data reveal that in the absence of a $M A X$ mutation, loss of the maternal copy of chromosome 14 is still crucial to the development of a subset of PPGL suggesting a role for gene imprinting.

In order to examine the evolution of PPGL the authors explored the relationship between a primary $S D H B$ PCC and a metastatic site in regards to mutations and copy-number events. They found that loss of $1 \mathrm{p}$ and $11 \mathrm{p}$ as well as mutations in SLMO1 and ELMOD3 were common between the primary and metastasis, suggesting these were early clonal events. The metastasis demonstrated additional loss of chromosomes 3 , $6 \mathrm{q}, 14$, and $17 \mathrm{p}$, and a gain of $17 \mathrm{q}$, as well as mutations in NDST3, DDX46, and TNXB that were absent in the primary. The primary also harboured mutations in GTPBP10 and TTC39C. These data, while illustrating that PPGL is a continually evolving tumour, provide little information on the degree of heterogeneity present within a primary tumour.

Further insight into intratumoural heterogeneity in PPGL was provided by a recent study performing analysis of somatic copy-number aberrations in 138 samples from 104 tumours in 94 patients (Crona et al., 2015). The authors performed either multiregional sampling or inferred heterogeneity based on genomic alterations present in a subclonal cellular fraction. Using bioinformatic inference the authors 
observed genomic heterogeneity in 74 of 136 (54\%) samples. In contrast, multiregional sampling demonstrated heterogeneity in 22 of 24 (92\%) patients analysed. As the authors note, using subclonality to infer heterogeneity may result in underestimation as, unless very large tumour portions are used, only colocalised clones will be detected. Perhaps the most important observation of the study was that, although not statistically significant, tumours from patients with malignant disease were characterised by a greater degree of genomic instability and intratumoural heterogeneity. While this study represents the first major examination of intratumoural heterogeneity in PPGL, moving forward a combination of basepair resolution technologies and multiregional sampling will be required to comprehensively map PPGL intratumoural heterogeneity.

\subsection{Goals of this Thesis}

In summation, there are currently as many as 18 genes known to act as primary drivers in the development of PPGL. Mutations in these genes, occurring either somatically or in the germline, account for between 70 and $80 \%$ of PPGL. The majority of remaining cases that cannot be attributed to one of the known drivers appear to have similar mRNA and miRNA profiles, suggesting a common mechanism of oncogenesis. Beyond the primary drivers, very little is known about the secondary pathways and mechanisms that are dysregulated in PPGL. Similarly, there is limited knowledge of the mutational and evolutionary processes that occur within PPGL or to what degree they exhibit subclonal heterogeneity.

An understanding of the auxiliary pathways involved in the oncogenesis of PPGL is vital for the development of novel treatments that target oncogenic dependencies within the tumour. As such the first aim of this research project was to explore and catalogue the genomic alterations in a cohort PPGL, in order to search for recurrently mutated genes and pathways, novel driver mutations in unexplained PPGL, and mutational signatures indicative of specific mutational processes (Alexandrov, Nik-Zainal, Wedge, Aparicio, et al., 2013).

In concert, this thesis aimed to extend the knowledge around PPGL subtypes by applying a consensus clustering approach to a compendium of published microarray data. The ultimate goal of this was to develop a clinically relevant classification tool for subtyping PPGL to assist with interpretation of genetic testing, guidance of clinical decision-making, and identification of high interest cases for further study.

Tumour heterogeneity is one of the greatest challenges facing modern cancer therapies and is thought to underpin acquired resistance to many frontline treatments (Turner \& Reis-Filho, 2012). Examination of subclonal heterogeneity can also provide insight into the obligate steps in oncogenesis through examples 
of convergent evolution, leading to a better understanding of tumour dependencies (McGranahan \& Swanton, 2015). In light of this, the final aim of this thesis was to assess both inter- and intratumoural heterogeneity in PPGL as a function of the evolutionary processes active in tumour development. 


\section{Materials and Methods}

\subsection{Ethical Considerations of Sample Acquisition}

De-identified patient samples were obtained with approval of the Human Research Ethics Committees at The Peter MacCallum Cancer Centre, The Kolling Institute of Medical Research, the Royal Melbourne Hospital, and the Sydney Children's Hospitals Network. Patient consent was obtained either prospectively, for samples obtained during the research period, or retrospectively, for samples obtained prior to the research period and not covered by existing consent. Where possible, informed consent was obtained by the treating physician, otherwise a member of the Victorian Cancer Biobank or the project's ethics coordinator, Annette Hogg, obtained consent. When available, patient clinical history and relevant pathology reports were obtained from the hospital of origin in a de-identified manner.

\subsection{In-vitro Methods}

Table 2.1 Reagent table

\begin{tabular}{|c|c|c|}
\hline Reagent & Manufacturer & Part Number \\
\hline Xylene & Sigma Aldrich & 247642-500ML \\
\hline HotStarTaq DNA Polymerase & Qiagen & 203203 \\
\hline Agarose & Promega & V3121 \\
\hline Ethidium Bromide & Invitrogen & $15585-011$ \\
\hline QIAzol Lysis Reagent & Qiagen & 79306 \\
\hline miRNeasy Mini Kit & Qiagen & 217004 \\
\hline RNeasy FFPE Kit & Qiagen & 73504 \\
\hline DNeasy Blood \& Tissue Kit & Qiagen & 69504 \\
\hline Chloroform & Sigma Aldrich & C2432 \\
\hline SureSelect XT All Exon Target Enrichment v5 & Agilent Technologies & $5190-6208$ \\
\hline SeqCap EZ Human Exome Library v2 & Nimblegen (Roche) & 05860504001 \\
\hline TruSeq RNA Sample Preparation Kit v2 & Illumina & RS-122-2001 \\
\hline TruSeq DNA Library Preparation Kit & Illumina & FC-121-2001 \\
\hline ThruPLEX-FD Prep Kit & Rubicon Genomics & R40048 \\
\hline CytoScan HD Array and Reagent Kit & Affymetrix & 901835 \\
\hline Ncounter GX Custom Codeset & Nanostring & NANGXA-P1CS-096 \\
\hline Ncounter Master Kit & Nanostring & NANNAA-AKIT-48 \\
\hline OncoScan FFPE Assay Kit & Affymetrix & 902293 \\
\hline QIAamp Circulating Nucleic Acid Kit & Qiagen & 55114 \\
\hline Agencourt AMPure XP Magnetic Beads & Beckman Coulter & A63880 \\
\hline Big Dye Terminator v3.1 & Applied Biosystems & 4337455 \\
\hline SuperScript II Reverse Transcriptase & Invitrogen & $18064-014$ \\
\hline Phusion High-Fidelity DNA Polymerase & New England Biolabs & M0530S \\
\hline Herculase II Fusion DNA Polymerase & Agilent Technologies & 600675 \\
\hline Dynabeads M-270 Streptavidin & Invitrogen & 65305 \\
\hline Dynabeads MyOne Streptavidin T1 & Invitrogen & 65601 \\
\hline
\end{tabular}




\subsubsection{Nucleic Acid Extraction}

\subsubsection{Total RNA Extraction from Fresh Frozen Tissue}

A portion $(10-30 \mathrm{mg})$ of frozen tissue was excised from the tumour specimen, placed in $1 \mathrm{~mL}$ of QIAzol (Qiagen, Germany) reagent, and mechanically disrupted using an Ultra-Turrex (IKA, Selangor, Malaysia) dispersing instrument. Chloroform $(200 \mu \mathrm{L})$ was added to the homogenised solution and mixed by agitation. The aqueous phase was separated by high-speed centrifugation and transferred to a clean 1.5 $\mathrm{mL}$ tube. Total RNA was then purified by column chromatography using an miRNeasy RNA extraction kit (Qiagen, Germany) in accordance with the manufacturer's protocol. Purified RNA was eluted in $50 \mu \mathrm{L}$ of RNase free water. RNA integrity was assayed using an Agilent 2100 Bioanalyzer (Agilent Technologies, CA, USA).

\subsubsection{Total RNA Extraction from FFPE Tissue}

Formalin-fixed paraffin-embedded (FFPE) tumour specimens were cut onto glass slides in $5 \mu \mathrm{m}$ sections. Tissue regions of interest were macro-dissected from the slide surface using a surgical blade and placed into a $1.5 \mathrm{~mL}$ tube. The tissue fragments were deparaffinised by incubation with $1 \mathrm{~mL}$ xylene. Tissue was recovered by high-speed centrifugation and removal of xylene supernatant, residual xylene was eliminated by incubation with $1 \mathrm{~mL}$ of absolute ethanol. RNA was extracted from deparaffinised tissue by column chromatography using an RNeasy FFPE extraction kit (Qiagen, Germany) in accordance with the manufacturer's protocol. Purified RNA was eluted in between 10 and $30 \mu \mathrm{L}$ of RNase free water depending on the quantity of starting material.

\subsubsection{DNA Extraction from Fresh Frozen Tissue}

A portion (10-30 mg) of frozen tissue was excised from the tumour specimen. The tissue was incubated with proteinase K in lysis buffer (Buffer ATL, DNeasy Blood and Tissue Kit, Qiagen) for three hours. DNA was purified from tissue lysis solution by column chromotography using a DNeasy Blood and Tissue DNA extraction kit (Qiagen, Germany) in accordance with the manufacturer's protocol. First and second elutions were performed with 200 and $100 \mu \mathrm{L}$ of $10 \mathrm{mM}$ Tris-Cl (Buffer EB, DNeasy Blood and Tissue Kit, Qiagen), respectively.

\subsubsection{DNA Extraction from FFPE Tissue}

FFPE tumour specimens were cut onto glass slides in $5 \mu \mathrm{m}$ sections. Tissue regions of interest were macro-dissected from the slide surface using a surgical blade and placed into a $1.5 \mathrm{~mL}$ tube. The tissue fragments were resuspended in $100 \mu \mathrm{L}$ of tissue lysis buffer (Buffer ATL, DNeasy Blood and Tissue Kit, 
Qiagen), incubated at $98^{\circ} \mathrm{C}$ for 15 minutes, and then allowed to cool for 5 minutes at room temperature. Proteinase $\mathrm{K}(12 \mu \mathrm{L})$ was added to tissue lysate. The solution was incubated for 72 hours with proteinase $\mathrm{K}$ being replenished every 24 hours. Lysis buffer $(111 \mu \mathrm{L}$, Buffer AL, DNeasy Blood and Tissue Kit, Qiagen) was added and the solution was incubated at $72^{\circ} \mathrm{C}$ for 10 minutes. Absolute ethanol $(111 \mu \mathrm{L})$ was added and the solution was passed through a chromatography column (DNeasy Mini spin column, DNeasy Blood and Tissue Kit, Qiagen). The column was washed with $277 \mu \mathrm{L}$ of each wash solution (Buffers AW1 and AW2, DNeasy Blood and Tissue Kit, Qiagen). Pre-warmed $\left(56^{\circ} \mathrm{C}\right)$ elution buffer $(50$ $\mu \mathrm{L}$, Buffer AE, DNeasy Blood and Tissue Kit, Qiagen) was applied to a column and let stand for 5 minutes. Purified DNA was eluted by high-speed centrifugation.

\subsubsection{Isolation of Circulating Cell-free DNA}

Peripheral blood $(10 \mathrm{~mL})$ was siphoned into a $\mathrm{K}_{2}$ EDTA vacutainer. The plasma fraction was separated by centrifugation at $1500 \mathrm{rpm}$ for 15 minutes at room temperature. The isolated plasma was further centrifuged at $4000 \mathrm{rpm}$ for 10 minutes at $4^{\circ} \mathrm{C}$ and the supernatant transferred to a new tube. Circulating nucleic acids were isolated from the purified plasma $(3 \mathrm{~mL})$ using a QIAamp Circulating Nucleic Acid Kit (Qiagen, Germany) in accordance with the manufacturer's protocol.

\subsubsection{Library Preparation}

\subsubsection{RNA-seq: Illumina TruSeq RNA V2}

RNA-seq libraries were prepared from $1 \mu \mathrm{g}$ of total RNA using anIllumina TruSeq RNA sample preparation (TS-RNA) kit version 2 (Illumina, CA, USA). Briefly, poly-A-tailed mRNA molecules were purified from total-RNA using oligo-dT coated magnetic beads then fragmented. First-strand cDNA synthesis was then performed using SuperScript II Reverse-Transcriptase (Invitrogen, Life Technologies, CA, USA) and first-strand master-mix (component FSM, TS-RNA kit). Reaction mixture was incubated at $25^{\circ} \mathrm{C}$ for 10 minutes, $42^{\circ} \mathrm{C}$ for 50 minutes, and $70^{\circ} \mathrm{C}$ for 15 minutes, then cooled to $4{ }^{\circ} \mathrm{C}$. Second-strand master-mix (component SSM, TS-RNA kit) was added and incubated at $16^{\circ} \mathrm{C}$ for 1 hour to produce double-stranded cDNA. Newly synthesised cDNA was purified using AMPure XP magnetic beads (Beckman Coulter, IN, USA). Overhanging ends were repaired by incubating double-stranded cDNA in end-repair master-mix (component ERP, TS-RNA kit) at $30^{\circ} \mathrm{C}$ for 30 minutes then bead purified. Purified end-repaired cDNA was added to A-tailing master-mix (component ATL, TS-RNA kit) and incubated at $37^{\circ} \mathrm{C}$ for 30 minutes then $70^{\circ} \mathrm{C}$ for 5 minutes. A-tailed cDNA was combined with ligation mix (component LIG, TS-RNA kit) and the desired indexed adapter (component AR0xx, TS-RNA kit), 
the reaction was incubated at $30^{\circ} \mathrm{C}$ for 10 minutes then purified using AMPure XP magnetic beads (Beckman Coulter, IN, USA). Ligated fragments were enriched by 15 cycles of PCR amplification $\left(98^{\circ} \mathrm{C}\right.$ for 30 seconds; $98^{\circ} \mathrm{C}$ for 10 seconds, $60^{\circ} \mathrm{C}$ for 30 seconds, $72^{\circ} \mathrm{C}$ for 30 seconds $(15 \mathrm{x}) ; 72^{\circ} \mathrm{C}$ for 5 minutes) using PCR master-mix (component PPM, TS-RNA kit) and primer-cocktail (component PPC, TS-RNA kit).

\subsubsection{DNA-seq: Illumina TruSeq DNA V2}

Libraries were prepared using anIllumina TruSeq DNA library preparation (TS-DNA) kit version 2 (Illumina, CA, USA) according to the manufacturer's protocol. Briefly, between $300 \mathrm{ng}$ and $1 \mu \mathrm{g}$ of input DNA was fragmented using a Covaris S2 sonicator (Covaris, MA, USA). Shearing parameters were as follows - duty cycle: $10 \%$, intensity: 5, cycles per burst: 200, duration: 3 x 60 seconds, mode: frequency sweeping, temperature: $6-8^{\circ} \mathrm{C}$. Overhanging ends were repaired by incubating fragmented DNA in endrepair master-mix (component ERP, TS-DNA kit) at $30^{\circ} \mathrm{C}$ for 30 minutes then bead purified. Purified end-repaired DNA was added to A-tailing master-mix (component ATL, TS-DNA kit) and incubated at $37^{\circ} \mathrm{C}$ for 30 minutes. A-tailed DNA was combined with ligation mix (component LIG, TS-DNA kit) and the desired indexed adapter (component AD0xx, TS-DNA kit), the reaction was incubated at $30^{\circ} \mathrm{C}$ for 10 minutes then purified using AMPure XP magnetic beads (Beckman Coulter, IN, USA). Post-ligation yield was quantitated using a Qubit fluorometric quantitation system (Life Technologies, CA, USA). Postligation DNA (200 ng) was enriched for ligated fragments by 6 cycles of PCR amplification $\left(98^{\circ} \mathrm{C}\right.$ for 30 seconds; $98^{\circ} \mathrm{C}$ for 10 seconds, $60^{\circ} \mathrm{C}$ for 30 seconds, $72^{\circ} \mathrm{C}$ for 30 seconds $(6 \mathrm{x}) ; 72^{\circ} \mathrm{C}$ for 5 minutes) using Phusion High-Fidelity DNA Polymerase (New England Biolabs, MA, USA) and primer cocktail (component PPC, TS-DNA kit). PCR products were purified using AMPure XP magnetic beads and library fragment distribution was quality controlled using an Agilent 2100 Bioanalyzer (Agilent Technologies, CA, USA).

\subsubsection{DNA-seq: Agilent SureSelect}

Between $300 \mathrm{ng}$ and $1 \mu \mathrm{g}$ of input DNA was fragmented as per section 2.2.2.2. Libraries were prepared using aSureSelect library preparation kit (SureSelect, Agilent Technologies, CA, USA). End-repair master-mix was prepared by combining 10x End Repair Buffer $(10 \mu \mathrm{L})$, dNTP Mix (1.6 $\mu \mathrm{L})$, T4 DNA Polymerase $(1 \mu \mathrm{L})$, Klenow DNA Polymerase $(2 \mu \mathrm{L})$, T4 Polynucleotide Kinase $(2.2 \mu \mathrm{L})$ and nucleasefree water $(35.2 \mu \mathrm{L})$. Fragmented DNA $(48 \mu \mathrm{L})$ and end-repair master-mix $(52 \mu \mathrm{L})$ were combined, incubated at $20^{\circ} \mathrm{C}$ for 30 minutes, then purified using AMPure XP magnetic beads (Beckman Coulter, IN, USA). Adenylation master-mix was prepared by combining $10 \times$ Klenow Polymerase Buffer $(5 \mu \mathrm{L})$, 
dATP $(1 \mu \mathrm{L})$, Exo(-) Klenow $(3 \mu \mathrm{L})$, and nuclease-free water $(11 \mu \mathrm{L})$. Adenylation master-mix $(20 \mu \mathrm{L})$ and end-repaired template $(30 \mu \mathrm{L})$ were combined, incubated at $37^{\circ} \mathrm{C}$ for 30 minutes, then purified using AMPure XP magnetic beads. Ligation master-mix was prepared by combining $5 \times$ T4 DNA Ligase Buffer $(10 \mu \mathrm{L})$, SureSelect Adaptor Oligo Mix $(2 \mu \mathrm{L})$, T4 DNA Ligase $(1.5 \mu \mathrm{L})$, and Nuclease-free water $(23.5$ $\mu \mathrm{L})$. Ligation master-mix $(37 \mu \mathrm{L})$ and adenylated template $(13 \mu \mathrm{L})$ were combined, incubated at $20^{\circ} \mathrm{C}$ for 15 minutes, then purified using AMPure XP magnetic beads. PCR master-mix was prepared by combining $5 \times$ Herculase II Reaction Buffer $(10 \mu \mathrm{L}), 100 \mathrm{mM}$ dNTP Mix $(0.5 \mu \mathrm{L})$, Herculase II Fusion DNA Polymerase $(1 \mu \mathrm{L})$ (Agilent Technologies, CA, USA), SureSelect Primer (1.25 $\mu \mathrm{L})$, SureSelect ILM Indexing Pre-Capture PCR Reverse Primer $(1.25 \mu \mathrm{L})$, and nuclease-free water $(6 \mu \mathrm{L})$. PCR master-mix $(20 \mu \mathrm{L})$ and adapter ligated template $(250 \mathrm{ng}$ in $20 \mu \mathrm{L})$ were combined. Post-ligation DNA was enriched for ligated fragments by 6 cycles of PCR amplification $\left(98^{\circ} \mathrm{C}\right.$ for 2 minutes; $98^{\circ} \mathrm{C}$ for 30 seconds, $65^{\circ} \mathrm{C}$ for 30 seconds, $72^{\circ} \mathrm{C}$ for 1 minute $(6 \mathrm{x}) ; 72^{\circ} \mathrm{C}$ for 10 minutes). PCR products were purified using AMPure XP magnetic beads and library fragment distribution was quality controlled using an Agilent 2100 Bioanalyzer (Agilent Technologies, CA, USA).

\subsubsection{Circulating Nucleic Acids: Rubicon ThruPLEX-FD}

Sequencing libraries were produced from $25 \mathrm{ng}$ of purified cell-free DNA using a ThruPLEX-FD library preparation kit (Rubicon Genomics, MI, USA). Briefly, template DNA $(10 \mu \mathrm{L})$ was combined with template preparation buffer $(2 \mu \mathrm{L})$ and template preparation enzyme $(1 \mu \mathrm{L})$. Reaction was incubated at $22^{\circ} \mathrm{C}$ for 25 minutes followed by $55^{\circ} \mathrm{C}$ for 20 minutes. Library synthesis buffer $(1 \mu \mathrm{L})$ and Library synthesis enzyme $(1 \mu \mathrm{L})$ were added and reaction was incubated at $22^{\circ} \mathrm{C}$ for 40 minutes. Library amplification buffer $(48.5 \mu \mathrm{L})$, library amplification enzyme $(1.5 \mu \mathrm{L})$, nuclease-free water $(8 \mu \mathrm{L})$, and indexing reagent $(2 \mu \mathrm{L})$ were added. The library was then enriched by PCR amplification $\left(72^{\circ} \mathrm{C}\right.$ for 3 minutes; $85^{\circ} \mathrm{C}$ for 2 minutes; $98^{\circ} \mathrm{C}$ for 2 minutes; $98^{\circ} \mathrm{C}$ for 20 seconds, $67^{\circ} \mathrm{C}$ for 20 seconds, $72^{\circ} \mathrm{C}$ for 40 second (4x); $98^{\circ} \mathrm{C}$ for 20 seconds, $72^{\circ} \mathrm{C}$ for 50 seconds (11x)). PCR products were purified using AMPure XP magnetic beads and library fragment distribution was quality controlled using an Agilent 2100 Bioanalyzer (Agilent Technologies, CA, USA).

\subsubsection{Exome Capture}

\subsubsection{Roche NimbleGen SeqCap EZ Human Exome}

Hybridisation-based target enrichment was performed using a NimbleGen SeqCap EZ Human Exome Library V2 kit (Nimblegen, Roche, WI, USA) and SeqCap Hybridization and Wash Kits (Nimblegen, 
Roche, WI, USA). When capture was to be performed in multiplex, libraries were pooled in equimolar amounts to a total of $750 \mathrm{ng}$ of DNA, otherwise $750 \mathrm{ng}$ of a single library was used. Single or multiplexed libraries were combined with $5 \mu \mathrm{g}$ of COT Human DNA and $2 \mathrm{nmol}$ of Hybridization Enhancing Oligos then dehydrated in a centrifugal evaporator. Dehydrated components were resuspended in hybridisation component $\mathrm{A}(3 \mu \mathrm{L})$ and $2 \mathrm{x}$ hybridisation buffer $(7.5 \mu \mathrm{L})$ and the mixture was incubated at $95^{\circ} \mathrm{C}$ for 10 minutes. The mixture was combined with the SeqCap EZ exome bait library $(4.5 \mu \mathrm{L})$ and incubated at $47^{\circ} \mathrm{C}$ for 72 hours with the thermocycler lid set to $57^{\circ} \mathrm{C}$. Following incubation, prewashed Dynabeads M-270 Streptavidin (Invitrogen, Life Technologies, CA, USA) magnetic beads $(100 \mu \mathrm{L})$ were added and the reaction was incubated at $47^{\circ} \mathrm{C}$ for 45 minutes to allow binding. After incubation, pre-warmed $\left(47^{\circ} \mathrm{C}\right)$ wash buffer $\mathrm{I}(100 \mu \mathrm{L})$ was added and the mixture was placed on a magnetic rack. The beads were washed once with pre-warmed stringent wash buffer $\left(47^{\circ} \mathrm{C}\right)$, once with room temperature stringent wash buffer, and once with each of wash buffers I, II, and III. Beads were resuspended in nuclease-free water $(50 \mu \mathrm{L})$. Captured library was amplified with 12 cycles of PCR $\left(98^{\circ} \mathrm{C}\right.$ for 45 seconds; $98^{\circ} \mathrm{C}$ for 15 seconds, $60^{\circ} \mathrm{C}$ for 30 seconds, $72^{\circ} \mathrm{C}$ for 30 seconds $(12 \mathrm{x}) ; 72^{\circ} \mathrm{C}$ for 60 seconds) using Phusion High-Fidelity DNA Polymerase (New England Biolabs, MA, USA). PCR products were purified using AMPure XP magnetic beads and library fragment distribution was quality controlled using an Agilent 2100 Bioanalyzer (Agilent Technologies, CA, USA).

\subsubsection{SureSelect XT Human All Exon}

Hybridisation-based target enrichment was performed using the SureSelect XT Human All Exon V5 Target Enrichment System (Agilent Technologies, CA, USA). Template library (750 ng) was dehydrated in a centrifugal evaporator and resuspended at $221 \mathrm{ng} / \mu \mathrm{L}$ in nuclease-free water. Blocking oligo mix was added and the mixture was incubated at $95^{\circ} \mathrm{C}$ for 5 minutes then held at $65^{\circ} \mathrm{C}$. SureSelect XT capture library baits $(5 \mu \mathrm{L})$ were combined with $25 \%$ RNase-block $(2 \mu \mathrm{L})$ and hybridisation buffer $(13 \mu \mathrm{L})$ then added to pre-heated template library. The reaction was incubated at $65^{\circ} \mathrm{C}$ for 24 hours with thermocycler lid set to $105^{\circ} \mathrm{C}$. Dynabeads MyOne Streptavidin T1 $(2 \mu \mathrm{L})$ were washed and resuspended in binding buffer $(200 \mu \mathrm{L})$. Hybridised library was added to streptavidin beads and the mixture was incubated for 30 minutes with agitation. The supernatant was removed from the beads using a magnetic separator, the beads were resuspended in wash Buffer $1(200 \mu \mathrm{L})$ and incubated for 15 minutes at room temperature. Following incubation, wash Buffer 1 was replaced with pre-warmed $\left(65^{\circ} \mathrm{C}\right)$ wash Buffer $2(200 \mu \mathrm{L})$ and mixture was incubated at $65^{\circ} \mathrm{C}$ for 10 minutes, this step was repeated once for a total of three washes. The beads were sequestered using a magnetic separator and resuspended in nuclease-free water $(30 \mu \mathrm{L})$. PCR master-mix was prepared by combining $5 \times$ Herculase II Reaction Buffer $(10 \mu \mathrm{L}), 100 \mathrm{mM}$ dNTP Mix 
$(0.5 \mu \mathrm{L})$, Herculase II Fusion DNA Polymerase $(1 \mu \mathrm{L})$ (Agilent Technologies, CA, USA), SureSelect ILM Indexing Post-Capture Forward PCR Primer $(1 \mu \mathrm{L})$, and nuclease-free water $(18.5 \mu \mathrm{L})$. The beadbound library $(14 \mu \mathrm{L})$ and an index-specific reverse primer $(5 \mu \mathrm{L})$ were combined with PCR master-mix $(31 \mu \mathrm{L})$. Target-enriched library was amplified by 12 cycles of PCR $\left(98^{\circ} \mathrm{C}\right.$ for 2 minutes; $98^{\circ} \mathrm{C}$ for 30 seconds, $57^{\circ} \mathrm{C}$ for 30 seconds, $72^{\circ} \mathrm{C}$ for 1 minute $(6 \mathrm{x}) ; 72^{\circ} \mathrm{C}$ for 10 minutes). PCR products were purified using AMPure XP magnetic beads and library fragment distribution was quality controlled using an Agilent 2100 Bioanalyzer (Agilent Technologies, CA, USA).

\subsubsection{Variant Validation}

\subsubsection{PCR Amplification, Verification, and Cleanup}

Template DNA (10 ng) was added to a reaction containing $0.1 \mu \mathrm{L}$ (0.5 Units) of HotStarTaq DNA Polymerase, $200 \mu \mathrm{M}$ dNTPs, $1.5 \mathrm{mM} \mathrm{MgCl}_{2}$, and $1 \mathrm{mM}$ sequence-specific primers in a total volume of $20 \mu \mathrm{L}$. The polymerase was activated by incubating the reaction at $94^{\circ} \mathrm{C}$ for 15 minute in a thermocycler. The reaction was then denatured at $94^{\circ} \mathrm{C}$ for 30 seconds, annealed at $68^{\circ} \mathrm{C}$ for 30 seconds, and extended at $72^{\circ} \mathrm{C}$ for 30 seconds. This cycle was repeated eight times reducing the annealing temperature by $1^{\circ} \mathrm{C}$ with each cycle. The annealing temperature was then fixed at $61^{\circ} \mathrm{C}$ and the reaction was cycled for an additional 27 cycles. A portion of the amplified product $(3 \mu 1)$ was visualised by electrophoreses on a $2 \%$ agarose gel containing ethidium bromide. The remaining amplified product was purified using Agencourt AMPure XP PCR purification reagent (Beckman Coulter, IN, USA).

\subsubsection{Sanger Dideoxynucleotide Sequencing}

Template DNA (10 ng) and 9.6 pmol of forward or reverse primer were combined in a total volume of 12 $\mu \mathrm{L}$. The primer template cocktail was submitted to the Australian Genome Research Facility for Sanger dideoxynucleotide sequencing using the Big Dye Terminator (BDT) chemistry version 3.1 (Applied Biosystems, MA, USA).

\subsubsection{Copy-number Arrays}

\subsubsection{Affymetrix Cytoscan HD}

Template DNA (500 ng) extracted from fresh frozen tissue was submitted to The Ramaciotti Centre for Genomics for processing with a CytoScan HD Array and Reagent Kit (Affymetrix, CA, USA) in accordance with the manufacturer's protocol. 


\subsubsection{Affymetrix Oncoscan}

Template DNA (100 ng) extracted from FFPE tissue was submitted to The Ramaciotti Centre for Genomics for processing with an Oncoscan FFPE Assay Kit (Affymetrix, CA, USA) in accordance with the manufacturer's protocol.

\subsubsection{Nanostring nCounter GX}

Expression levels for a panel of 52 genes were determined using the nCounter GX gene-expression system (Nanostring Technologies, WA, USA) in accordance with the manufacturer's protocol. Briefly, RNA isolated from fresh frozen tissue (100 ng) or FFPE tissue (300 ng) was combined with Reporter CodeSet $(10 \mu \mathrm{L})$, hybridisation buffer $(10 \mu \mathrm{L})$, and Capture ProbeSet $(5 \mu \mathrm{L})$. The mixture was incubated at $65^{\circ} \mathrm{C}$ for 12 hours. Post-hybridisation processing was performed using a nCounter Prep Station (Nanostring Technologies, WA, USA) and reporter probe counts were assayed on a Nanostring Digital Analyser (Nanostring Technologies, WA, USA).

\subsubsection{Immunohistochemistry}

\subsubsection{Chromogranin A}

Four $\mu \mathrm{m}$ sections were cut for each tumour, dewaxed in xylene and rehydrated through a graded ethanol series. Appropriate control tissues were used to verify the quality of each stain. Staining for chromogranin A was performed using a Roche Ventana Ultra immunostainer (Ventana Medical Systems). Antigen retrieval involved incubation with Ventana Ultra CC1 Conditioning Solution for 32 minutes. Chromogranin A primary antibody (Dako M0869 Clone DAK-A3) was diluted 1:400 with Ventana Antibody Dilution Buffer and incubated at $36^{\circ} \mathrm{C}$ for 20 minutes. Antibody detection was performed using Ventana OptiView DAB IHC Detection kit and slides were counterstained with haematoxylin. Protocol performed by Peter MacCallum Cancer Centre Department of Pathology.

\subsubsection{SDHB}

Immunohistochemistry for SDHB was performed using a Leica BondIII autostainer (Leica Biosystem, VIC, Australia). For SDHB a mouse monoclonal antibody (clone 21A11, ABCAM ab14714) was used at a dilution of 1 in 500. Antibody detection was performed using a biotin-free Bond Polymer Defined Detection System (DS9713, Vision Biosystems, VIC, Australia), according to the manufacturer's protocol. Protocol performed by Royal Northshore Hospital Pathology department of pathology. 


\subsection{In Silico Methods}

\subsubsection{Short-read Alignment and Variant Calling}

Basecall data were processed by Illumina CASAVA suite to produce index-separated FASTQ files. Sequencing reads were aligned to the hg19 reference genome using BWA-mem (exome-seq) (Li \& Durbin, 2009) or bowtie-2 via TopHat (RNA-seq) (Trapnell et al., 2009). Local realignment around insertions and deletions (INDELs) was performed using GATK (McKenna et al., 2010), and duplicate reads removed using Picard. Single-nucleotide variants were detected using multiple variant callers. Two variant callers based on Bayesian probability estimation, muTect (Cibulskis et al., 2013) and SomaticSniper (Larson et al., 2012), and one based on generative probabilistic models, JointSNVMix (Roth et al., 2012), were applied to paired analysis of tumour and normal data. One Bayesian caller, GATK UnifiedGenotyper (McKenna et al., 2010), was applied to tumour and normal data in an unpaired analysis, and a single caller based on heuristic models, VarScan2 (Koboldt et al., 2009), was applied to tumour data alone. Insertions and deletions were detected using IndelGenotyper (McKenna et al., 2010) and VarScan2. High-confidence variants were those called by muTect or at least two other callers, with variant allele frequency greater than $10 \%$ of reads and supported by reads in both directions, excluding any missense changes not seen in the RNA-seq where at least 20 reads were present, and non-pathogenic SNVs seen in the germline of more than three other samples. Sequence variants were annotated via the Ensembl Perl application programming interface (Release 64). Additional gene annotation was sourced from the NCBI Entrez and UniProt databases.

\subsubsection{Copy-number Analysis}

\subsubsection{Array-based Copy-number Analysis}

Preliminary data analysis and visualisation was performed using Chromosome Analysis Suite (Affymetrix, CA, USA). Additional data normalisation and analysis was performed using Nexus Copynumber (BioDiscovery, CA, USA). Data were normalised using the supplied internal NCN reference generated from HapMap pooled samples. If required, diploid regions were assigned by assessing the combination of B-allele frequency (BAF) and copy state. The ASCAT algorithm was used to make an approximation of tumour purity (Van Loo et al., 2010). All other analyses were performed using the SNPFASST2 algorithm. In order to minimise the effect of signal noise, segmentation data were filtered to exclude segments containing less than 150 probes. Normalised probe data were extracted from CYCHP and OSCHP file using Affymetrix power tools for further analysis and visualisation using the R statistical computing framework. 


\subsubsection{Exome Seq-based Copy-number Analysis}

ADTEx (Amarasinghe et al., 2014), an algorithm that utilises hidden Markov models in combination with discrete wavelet transformation based denoising to derive copy-number alterations, was applied to exome sequencing data and the output was visualised using the R statistical computing framework.

\subsubsection{Gene-expression Analysis}

\subsubsection{Microarray}

\subsection{Amalgamating Published Gene-expression Data}

Microarray data sets were obtained from online data repositories (GEO accessions GDS2113 and GSE19987, and ArrayExpress accession E-MTAB-733). Array quality was assessed using Normalized Unscaled Standard Error (NUSE) plots. Outlier arrays were excluded from further analysis. The virtualArray R package (Heider \& Alt, 2013) was used to combine the arrays into a single data superset.

\subsection{Consensus-Clustering}

The ConsensusClusterPlus (Wilkerson \& Waltman, 2013) package for R was used to assess the most stable number of clusters within the merged gene-expression data set using the 1000 most variable genes (pItem: 0.7; ClusterAlg: HC; distance: Pearson; reps: 1000; maxK: 10). An appropriate cut-off of 0.65 for the item consensus metric was determined by obtaining the threshold value at which all arrays had affinity for a single class. Arrays that did not have affinity for any one class greater than 0.65 were assigned the unclassified state.

\subsection{Differential Gene-Expression Analysis}

Following consensus-clustering, the Affymetrix and limma (Smyth, 2005) R packages were used to perform one-versus-one all pairs differential gene expression analysis to determine the genes most representative of each class. The results were filtered for genes with a Benjamini-Hochberg-Yekutieli FDR-corrected p-value less than 0.05 and ranked by fold-change. A primary gene-list was derived by combining the top 100 genes resulting from each one-versus-one comparison and removing duplicate genes, yielding a list of 499 genes, henceforth referred to as the long classification list (LCL).

In order to create a more succinct gene-list for use with the nCounter GX platform, a second gene-list was derived from the intersection of genes represented in both one-versus-one and one-versus-rest differential gene-expression analysis. The results were filtered for genes with a fold-change greater than one and ranked by $\mathrm{p}$ value. For each class, the top 10 genes from each of the five one-versus-one comparisons 
were combined and the result was intersected with the top 50 genes derived from a one-versus-rest comparison for the respective class. Seven genes were randomly selected from the result of the intersection as representative for each class. In cases where the intersection did not yield at least seven genes, additional genes were selected from the relevant one-versus-rest comparison. If the gene-list for any class was ostensibly deficient in differential genes for any one contrasting class, additional genes were selected from the relevant one-versus-one comparison. The final gene-list contained 47 genes and is henceforth referred to as the short classification list (SCL).

\subsubsection{4 $k$-Nearest Neighbour $(k$-NN) Classification for Subtyping}

The $k$-NN algorithm, as present in the Class package for R, performs classification by majority vote using the $\mathrm{k}$ nearest training set members as determined by Euclidean distance. To train the classifier, the class labels derived from consensus clustering were used in conjunction with the genes in the LCL gene-list. Expression values for the training genes were extracted from the microarray superset then standardised to Z-scores. K, or the number of neighbours considered, was set to the nearest integer to the square root of the number of genes used for training.

\subsection{Support Vector Machine (SVM) Classification for Subtyping}

Classification was performed using a SVM operated in linear classification mode (C-type) as implemented in the e1071 library for R. Multiclass classification was performed using k(k-1)/2 (where k is the total number of classes) binary classifiers in a one-versus-one approach with the appropriate class found by a voting scheme.

\subsubsection{RNA-Seq Analysis}

\subsection{RNA-Seq Feature Counting}

Raw RNA-seq reads were summarised to feature counts using the HTSeq software package. Transcript information was based on the Genome Reference Consortium Human genome build 37 (GRCh37) retrieved from the Ensembl database (version 69) in Gene Transfer Format (GTF). Feature counts were normalised for sequencing depth to log counts per million (log-cpm) using the EdgeR (Robinson et al., 2010) package.

\subsection{Fusion Analysis}

Fusion transcripts were detected using the DeFuse software package (McPherson et al., 2011). Reads were aligned to Ensembl hg19 genome build GRCh37.62 and gene model GRCh37.69. All alignment and filtering parameters were left as default. The initial output was further filtered to remove read-through 
transcripts, fusions between genes less than $100 \mathrm{~kb}$ apart, fusion events occurring in more than one sample, and fusions predicted with a probability score of less than 0.75 . Furthermore, a $Z$-score was calculated for the expression of each gene participating in a fusion event relative to other samples in the cohort; fusion events in which the sum of the $Z$-scores for each partner was less than 1 were excluded.

\subsection{Classification of Samples Using RNA-seq Data}

Log-cpm values were extracted for genes in the LCL gene-list and standardised to Z-scores using the R statistical computing framework. Standardised expression data were input as the testing set into the k-NN classifier described in section 2.3.3.1.4.

\subsubsection{Nanostring nCounter GX}

\subsection{Probe Design}

As described previously, a list of 47 genes was derived through differential gene-expression analysis (see section 2.3.3.1.3). An additional 6 genes were selected as normalisation controls based on their having a low variation coefficient across all classes. The list of 53 genes was submitted to Nanostring Technologies for probe design. Probes were successfully designed for 51 genes, 2 genes were determined to have high sequence homology preventing unique probe design, a single probe was designed to target both genes.

\subsection{Data Normalisation}

Raw probe counts were obtained from the Nanostring Digital Analyser (Nanostring Technologies, WA, USA). To account for technical and sample variation experimental probes were normalised against positive control and housekeeping gene probes. The geometric mean of the positive control probes for each sample was determined to create a summarised-positive-control value (SPCV). A sample-scalingfactor (SSF) was determined for each sample by dividing the geometric mean of the SPCVs of all samples by each sample's SPCV. All experimental and housekeeping probes for each sample were multiplied by the respective SSF. Using the positive control scaled data, this process was repeated using the housekeeping gene controls in place of positive-control probes.

\subsection{Classification of Samples Using nCounter GX Data}

Normalised probe counts were standardised to Z-scores using the R statistical computing framework. Standardised data were input as the testing set into the linear SVM classifier described in section 2.3.3.1.5 using the SCL gene set. 


\subsubsection{Analysis of Subclonal Architecture}

The PyClone statistical model and software tool (Version 0.12.3) was used to infer clonal population structure within tumours subjected to multi-region sampling. The variant allele frequencies of highconfidence variants occurring in one or more regions of the tumour were supplied to the algorithm. The software was operated with variant priors determined by total copy-number. See Appendix A for complete configuration file.

\subsubsection{Allelic Enrichment Analysis}

In order to determine if identical or opposing allelic versions were affected by a chromosomal aberration, the variant allele frequency (VAF) of germline SNPs were compared between two samples from the same patient exhibiting identical chromosomal alterations. To do so, germline SNPs were called using the GATK UnifiedGenotyper, the results were filtered for variants with a read-depth greater than 50 in both tumour and normal, a VAF between 0.45 and 0.55 in the germline, and a valid dbSNP identifier. The VAF for SNPs passing filter in both tumour samples were plotted against one another. A positive or negative correlation indicates identical and opposing chromosomal copies affected, respectively. 


\section{The Genomic Landscape of Phaeochromocytoma}

\subsection{Chapter Preface}

This chapter, presented in the form of a peer-reviewed publication, represents the first exploration of the genomic landscape of PPGL. Although the previous two decades have seen a spectacular increase in the understanding of the primary genetic drivers of PPGL, at the inception of this project there remained a paucity of knowledge regarding the genomic landscape and the contribution of secondary genomic alterations to tumourigenesis. To address this deficiency, high-resolution genomics technologies were used to catalogue the single base changes, insertions and deletions, chromosomal copy-number alterations, and gene fusion events that contribute to the development and evolution of PPGL.

The seminal work by Dahia et al. (Dahia et al., 2005), and further refinement by Burnichon et al. (Burnichon et al., 2011), demonstrated the value and importance of defining gene-expression subtypes to refining our understanding PPGL biology. As such, part of the work presented in this chapter sought to expand and refine the current understanding of PPGL disease subtypes through a systematic metaanalysis of previously published gene-expression data, and in doing so develop a cross platform classification tool for clinically subtyping PPGL. 


\title{
The genomic landscape of phaeochromocytoma
}

\author{
Aidan Flynn,,,2 Diana Benn,,3 Roderick Clifton-Bligh,,,4 Bruce Robinson, ${ }^{3,4}$ Alison H Trainer,,,5 Paul James,' \\ Annette Hogg,' Kelly Waldeck,' Joshy George, ${ }^{6}$ Jason Li,' Stephen B Fox,' Anthony J Gill,4,7 Grant McArthur, ${ }^{1,8}$ \\ Rodney J Hicks ${ }^{1,8}$ and Richard W Tothill',2* \\ The Peter MacCallum Cancer Centre, East Melbourne, VIC, Australia \\ 2 The Department of Pathology, University of Melbourne, Parkville, VIC, Australia \\ 3 Cancer Genetics, Kolling Institute of Medical Research, Royal North Shore Hospital, Sydney, NSW, Australia \\ 4 University of Sydney, Sydney, NSW, Australia \\ 5 Royal Melbourne Hospital and Department of Medicine, University of Melbourne, Parkville, VIC, Australia \\ 6 Jackson Laboratory for Genomic Medicine, Farmington, CT 06030, USA \\ 7 Cancer Diagnosis and Pathology Group, Kolling Institute of Medical Research and Department of Anatomical Pathology, Royal North Shore \\ Hospital, Sydney, NSW, Australia \\ 8 The Sir Peter MacCallum Department of Oncology, University of Melbourne, Parkville, VIC, Australia
}

*Correspondence to: Richard W Tothill, The Peter MacCallum Cancer Centre, East Melbourne, VIC, Australia. E-mail: richard.tothill@petermac.org

\begin{abstract}
Phaeochromocytomas (PCCs) and paragangliomas (PGLs) are rare neural crest-derived tumours originating from adrenal chromaffin cells or extra-adrenal sympathetic and parasympathetic tissues. More than a third of PCC/PGL cases are associated with heritable syndromes involving 13 or more known genes. These genes have been broadly partitioned into two groups based on pseudo-hypoxic and receptor tyrosine kinase (RTK) signalling pathways. Many of these genes can also become somatically mutated, although up to one third of sporadic cases have no known genetic driver. Furthermore, little is known of the genes that co-operate with known driver genes to initiate and drive tumourigenesis. To explore the genomic landscape of PCC/PGL, we applied exome sequencing, high-density SNP-array analysis, and RNA sequencing to 36 PCCs and four functional PGL tumours. All tumours displayed low mutation frequency, in contrast to frequent large segmental copy-number alterations, aneuploidy, and evidence for chromothripsis in one case. Multi-region sampling of one benign familial PCC tumour provided evidence for the timing of mutations during tumourigenesis and ongoing clonal evolution. Thirty-one of 40 $(77.5 \%)$ cases could be explained by germline or somatic mutations or structural alterations affecting known PCC/PGL genes. Deleterious somatic mutations were also identified in known tumour-suppressor genes associated with genome maintenance and epigenetic modulation. A multitude of other genes were also found mutated that are likely important for normal neuroendocrine cell function. We revisited the gene-expression subtyping of PCC/PGL by integrating published microarray data with our RNA-seq data, enabling the identification of six robust gene-expression subtypes. The majority of cases in our cohort with no identifiable driver mutation were classified into a gene-expression subtype bearing similarity to $M A X$ mutant PCC/PGL. Our data suggest there are yet unknown PCC/PGL cancer genes that can phenocopy MAX mutant PCC/PGL tumours. This study provides new insight into the molecular diversity and genetic origins of PCC/PGL tumours.

Copyright (c) 2014 Pathological Society of Great Britain and Ireland. Published by John Wiley \& Sons, Ltd.
\end{abstract}

Keywords: phaeochromocytoma; paraganglioma; genomics; next-generation sequencing; mutations; gene-expression profiling; tumour heterogeneity

Received 21 September 2014; Revised 19 November 2014; Accepted 17 December 2014

No conflicts of interest were declared.

\section{Introduction}

Phaeochromocytomas (PCCs) and paragangliomas (PGLs) are rare tumours of neural crest origin arising from the adrenal medulla and extra-adrenal chromaffin tissues, respectively. PGL is a term also used to describe tumours of non-chromaffin tissue from parasympathetic ganglia of the head and neck [1]. PCC and PGL tumours of chromaffin tissues secrete catecholamines, 'fight or flight' hormones that cause debilitating hypertensive symptoms and potentially life-threatening cardiovascular conditions [2]. Although most PCC/PGL tumours are benign and cured by surgery, more than $10 \%$ of cases develop disseminated disease $[3,4]$. Patients with metastatic PCC/PGL have a poor outcome, with few durable treatment options.

PCC/PGL is a highly heritable form of human neoplasia and has been the subject of intensive genetic research. Approximately $40 \%$ of cases are associated 
with familial syndromes involving autosomal dominant mutations in at least 13 susceptibility genes [5]. Gene-expression profiling has been used to cluster the tumours into two or more subtypes correlating to genetic drivers and their dysregulated pathways [6-8]. The so-called pseudo-hypoxia cluster ('cluster 1') corresponds to oxygen-independent stabilization of hypoxia-inducible factors (HIFs) and activation of downstream oncogenic signalling. Mutated genes in this group include post-translational regulators of HIF stability such as VHL and in rare instances, the prolyl hydroxylase PHD2 (EGLNI) [9,10]. Alternatively, mutations in metabolic Krebs-cycle members, including the four succinate dehydrogenase subunits (SDHA-SDHD), SDH-flavination factor (SDHAF2), and fumarate hydratase $(F H)$, lead to accumulation of intracellular metabolites that inhibit the function of dioxygenase proteins such as PHD2 [11-17]. Stabilizing mutations in HIF2a (EPAS1) can also disrupt the binding of PHD proteins [18]. The second major gene-expression subtype ('cluster 2') comprises genes principally associated with receptor tyrosine kinase (RTK) and downstream signalling activity including RET, NF1, TMEM127, MAX, and KIF1B [19-24]. Somatic mutations in known PCC/PGL familial cancer genes and HRAS can also arise in sporadic cases [6,25-32]. In total, a pathogenic driver mutation can be found in more than $60 \%$ of all PCC/PGL cases.

Although much is known of primary driver genes in PCC/PGL, less is known of the co-operative somatic events required for cancer initiation and progression. Recurrent copy-number alterations have been documented in PCC/PGL, mainly regions of copy-number loss but also a few regions of gain [33-36]. Segmental losses involving PCC/PGL tumour-suppressor genes are frequently observed in tumours, resulting in biallelic inactivation [6]. However, these and other recurrent copy-number alterations also encompass other genes likely important for oncogenic transformation. Identifying single co-operative genes within regions of copy-number change in PCC/PGL is, however, difficult as there are few focally amplified or deleted regions; rather, whole chromosomes or chromosomal arms tend to be altered.

Massively parallel sequencing (MPS) has now been used to characterize many cancer types, unveiling the repertoire of genes implicated in tumourigenesis [37]. Additionally, the technology has been used to characterize mutational processes operative in tumour cells and to explore clonal evolution and heterogeneity $[38,39]$. MPS has recently been used for identifying new $\mathrm{PCC} / \mathrm{PGL}$ driver genes in small numbers of PCC/PGL samples and for demonstrating the clinical utility for genetic testing in this patient group $[16,18,22,40]$. However, to date, no large-scale sequencing efforts of PCC/PGL have been reported. To characterize the genomic landscape of PCC/PGL, we applied an integrated genomic analysis to 40 tumours to search for new driver and co-operative genes and to explore any associations with gene-expression subtypes within this disease group.

\section{Materials and methods}

\section{Sample collection}

Tumour and paired normal biospecimens [blood or normal formalin-fixed, paraffin-embedded (FFPE) tissue] were obtained from the Kolling Institute Neuroendocrine Tumour Bank $(n=25)$ and Victorian Cancer Biobank $(n=15)$. Patient consent and Institutional Review Board approval were obtained according to the guidelines of the Australian National Health and Medical Research Council (see Supplementary Table 1 for patient details).

\section{Exome and RNA sequencing}

DNA was extracted from fresh frozen tissue (all tumours), blood $(n=29)$, or FFPE tissue $(n=11)$ by column chromatography (Qiagen, Germany) and fragmented using a Covaris S2 sonicator (Covaris, MA, USA). Libraries were prepared using $300 \mathrm{ng}$ to $1 \mu \mathrm{g}$ of input DNA; 30 tumour/normal pairs were prepared using the IlluminaTruSeq V2 DNA library preparation kit (Illumina, CA, USA) and nine using the SureSelect library preparation kit (SureSelect, Agilent, CA, USA). Libraries were captured using the Nimblegen V2 (Nimblegen, Roche, WI, USA) or the Agilent SureSelect V5 exome capture platform (Supplementary Table 2). RNA was extracted using the Qiagen miRNAeasy kit and quality assessed using the Agilent 2100 Bioanalyzer (Agilent Technologies, CA, USA). RNA-seq libraries were prepared from $1 \mu \mathrm{g}$ of total RNA using the Illumina Truseq V2 RNA-seq kit. Sequencing was performed on the Illumina HiSeq2000 platform as paired-end $100 \mathrm{bp}$ runs according to the standard protocol.

\section{Short-read alignment and variant calling}

Sequencing reads were aligned to the hg19 reference genome using BWA-mem (exome-seq) [41] or bowtie-2 via TopHat (RNA-seq) [42]. Local realignment around insertions and deletions (InDels) was performed using GATK [43], and duplicate reads were removed using Picard. Single-nucleotide variants and InDels were detected using GATK UnifiedGenotyper and IndelGenotyper [43], muTect [44], SomaticSniper [45], VarScan [46], and JointSNVMix [47]. Sequence variants were annotated via the Ensembl Perl application programming interface (Release 64). Additional gene annotation was sourced from the NCBI Entrez and UniProt databases. High confidence variants were those called by muTect or at least two other callers, with variant allele frequency greater than $10 \%$ of reads and supported by reads in both directions, excluding any missense changes not seen in the RNA-seq where at least 20 reads were present, and non-pathogenic SNVs seen in the germline of more than three other samples. 


\section{Copy-number analysis}

High-resolution SNP genotyping and copy-number analysis were performed using Affymetrix Cytoscan HD arrays (Affymetrix, CA, USA). SNP-array data normalization and analysis were performed using Nexus Copy Number (BioDiscovery, CA, USA). Data were normalized using the supplied internal NCN reference generated from HapMap pooled samples. If required, diploid regions were assigned by assessing the combination of B-allele frequency and copy state. The ASCAT algorithm was used to make an approximation of tumour purity [48]. All other analyses were performed using the SNP-FASST2 algorithm. In order to minimize the effect of signal noise, segmentation data were filtered to exclude segments containing less than 150 probes. Copy-number alterations were called from exome-seq data using ADTEx [49].

Primary genomic data: Affymetrix Cytoscan HD array data are available through NCBI GEO (http://www.ncbi.nlm.nih.gov/geo/, GEO Accession ID: GSE61594).

Consensus clustering, cross-platform classification, and differential gene-expression analysis

Microarray datasets were obtained from online data repositories (GEO accessions GDS2113, GSE19987, and GSE19422; ArrayExpress accession E-MTAB-733). Array quality was assessed using Normalized Unscaled Standard Error (NUSE) plots. Outlier arrays were excluded from further analysis. The virtualArray $R$ package [50] was used to combine the arrays into a single dataset. The ConsensusClusterPlus [51] package for $\mathrm{R}$ was used to assess the most stable number of clusters within the merged dataset using the 1000 most variable genes (pItem: 0.7; ClusterAlg: HC; distance: Pearson; reps: 1000; maxK: 10). An appropriate cut-off of 0.65 for the item consensus metric was determined by obtaining the threshold value at which all arrays had affinity for a single class. Arrays that did not have affinity for any one class greater than 0.65 were assigned the unclassified state and excluded from use in training the classifier.

A one-versus-one all pairs differential gene expression analysis was performed using the Affymetrix and limma [52] $\mathrm{R}$ packages to determine the genes most representative of each class. The top 100 genes resulting from each comparison were combined and duplicate genes removed, yielding 499 genes (Supplementary Table 3). Mean centred and scaled expression values were used to train a $k$-nearest neighbour $(k-\mathrm{NN})$ classifier $(k=22)$ and test using an independently published microarray dataset (pre-normalized data used).

Raw RNA-seq reads were converted to feature counts using the HTSeq software package and then normalized for sequencing depth using the EdgeR [53] package, yielding log counts per million (log-cpm). The $k-\mathrm{NN}$ classifier was then applied to mean centred and scaled log-cpm expression values using the Class library for $\mathrm{R}$.

\section{Fusion analysis}

Fusion transcripts were detected using the DeFuse software package [54]. Reads were aligned to Ensembl hg19 genome build GRCh37.62 and gene model GRCh37.69. All alignment and filtering parameters were left as default. The initial output was further filtered to remove read-through transcripts, fusions between genes less than $100 \mathrm{~kb}$ apart, fusion events occurring in more than one sample, and fusions predicted with a probability score of less than 0.75 . Furthermore, a $Z$-score was calculated for the expression of each gene participating in a fusion event relative to other samples in the cohort; fusion events in which the sum of the $Z$-scores for each partner was less than 1 were excluded. Fusions were validated by PCR amplification from cDNA using unrelated samples as negative controls.

\section{Western blot analysis}

Western blotting using ALK antibody (Cell Signaling, Danvers, MA, USA; Cat No 3633) was performed using fresh-frozen tissue lysates as previously described [55].

\section{Results}

\section{The genomic landscape of PCC/PGL}

A cohort of 36 PCCs and four functional PGL tumours were analysed by exome-seq and high-density SNP-array analysis. RNA-seq was also applied to the tumour samples, with the exception of one tumour where RNA was of insufficient quality. Exome-seq and RNA-seq were applied to a high depth (Exome-seq, 120x; RNA-seq, 60-80 million reads), enabling comprehensive coverage of gene targets including the known PCC/PGL cancer genes (Supplementary Table 4). Tumour purity assessed using SNP-array data combined with somatic variant allele frequency (VAF) indicated that all samples had at least 50\% tumour content (Supplementary Table 5).

Analysis of the genomic data provided a rich survey of gene mutations, copy-number changes, and gene fusions in PCC/PGL (Figure 1A and Supplementary Tables 6-9). For the detection of single-nucleotide variants (SNVs), small insertions, and deletions (InDels), we used a combination of variant callers and applied post-hoc filtering to minimize false-positive variant calls (see the Materials and methods section). A subset of all variants $(n=204)$ was validated by Sanger sequencing, demonstrating a true-positive rate in the filtered list of $84 \%$. We subsequently used all variants passing filter plus any Sanger sequencing validated variants that did not pass filter for analysis and interpretation. Tumours had a variable but typically low mutation frequency of between four and 35 mutations in coding regions (Figure 1B). We pooled SNVs from all cases to assess the cumulative mutation signature in PCC/PGL (Figure 1C). The most frequent observed change involved $\mathrm{C}>\mathrm{T}$ transitions, a process commonly 


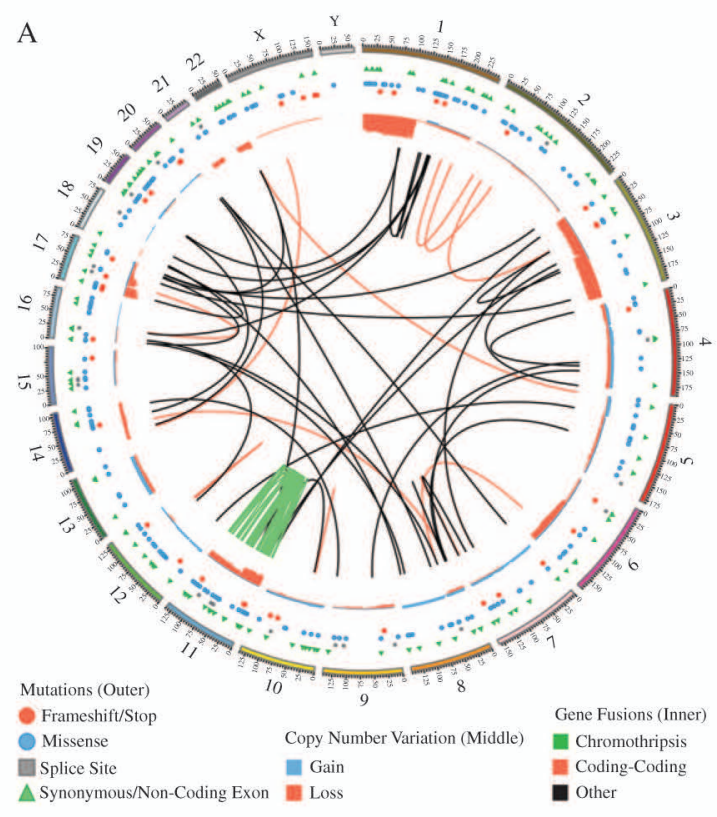

B

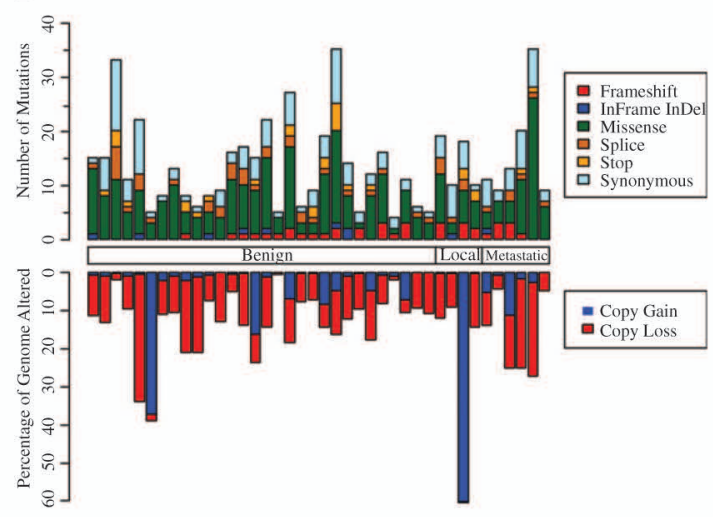

$\mathrm{C}$

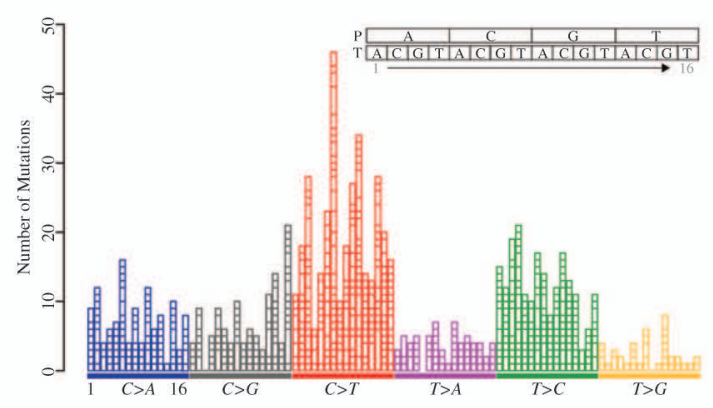

Figure 1. Genetic landscape of PCC/PGL. (A) Circos plot showing the combined distribution of subtle mutations, copy-number alterations, and transcript fusions. Fusions marked in red represent gene exon to exon partners; those marked in black represent gene exon to intergenic non-coding regions; and those marked in green highlight the fusions resulting from a chromothripsis event in case VCB02. (B) Histogram showing the total mutation load for each tumour colour-coded for the type and predicted mutational consequences (upper), and the percentage of each genome altered by chromosomal alterations (lower). (C) Mutational signature histogram showing the cumulative distribution of somatic single base substitutions across 40 samples. Mutations are classified by the base substitution as shown and ranked by mutational contexts considering the preceding $(\mathrm{P})$ and trailing $(\mathrm{T})$ base as shown in the key inset. As the mutation frequency in individual samples was insufficient for this analysis, the combined mutation data for the cohort were used with the contribution of each sample to the signature shown in stacked blocks. associated with ageing [38]. We observed a modest positive correlation between the patient age at surgery and the somatic variant frequency across the cohort (Supplementary Figure 1). We found no significant difference in mutation frequency between benign, locally invasive, and metastatic tumours, although sample number was likely underpowered for such an analysis.

Genome-wide copy-number analysis of tumours identified common regions of gain and loss, with a pattern similar to that previously reported in PCC/PGL [35,36] (Figure 1 and Supplementary Figure 2). Each tumour had on average $11.4 \%$ of the genome affected by large chromosomal losses and $4.6 \%$ affected by chromosomal gains; however, this figure is upwardly skewed by two outlier cases exhibiting $38 \%$ and $62 \%$ of the genome affected by gains. We applied fusion detection to the RNA-seq data and found evidence for interand intra-chromosomal breakpoints, many of which corresponded to edges of regions of loss or gain. One case (VCB02) displayed chromothripsis, characterized by a shattered pattern of microdeletions in chromosome 11 and clustering of intrachromosomal fusions from the RNA-seq data (Figure 1 and Supplementary Figure 3) [56]. Another case (VPH17) appeared to have no copy-number alterations (Supplementary Figure 4).

\section{Intratumoural heterogeneity}

To assess intratumoural heterogeneity in PCC, we multi-region sampled tissue from two opposing poles of a benign PCC tumour surgically resected from a 31-year-old female patient with a constitutional RET (p.C634Y) mutation (case P010) (Figure 2A). Copy-number analysis revealed a similar profile between the two tumour segments, with the exception of a single copy loss of chromosome 21 in the left pole and a focal amplification on chromosome 5 encompassing the genes KCTD16 and YIPF5 in the right pole (Figure $2 \mathrm{~B}$ ). Exome-seq revealed only 3 of 13 subtle mutations to be shared between tissue samples (Figure 2C), suggesting that most subtle mutations were acquired late in tumourigenesis. However, the VAF was relatively high $(>30 \%)$ for most private variants in each pole, indicating that the majority of cells in respective tumour segments harboured these mutations and represented divergent clonal outgrowths.

\section{A survey of known and putative cancer genes}

Germline or somatic mutations in known PCC/PGL cancer genes were identified in 31 of 40 samples (familial, 16; sporadic, 15) (Figure 3A and Supplementary Table 10). All germline mutations in VHL, SDHA, SDHB, and $R E T$ previously identified in genetic testing laboratories were detected in the exome-seq data $(n=14)$. An additional germline RET (Y791F) variant was identified in one patient who presented with unilateral benign PCC at 76 years of age, although the pathogenicity of RET codon 791 variants is controversial [57-59]. Careful examination of RNA-seq data identified a nonsense 
A

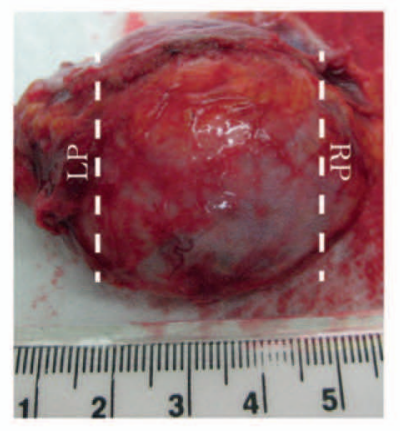

B

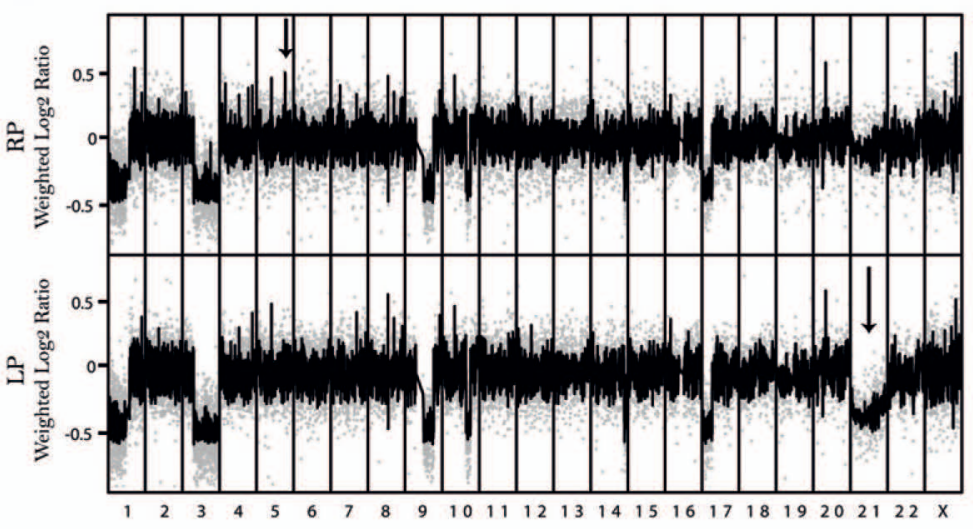

$\mathrm{C}$

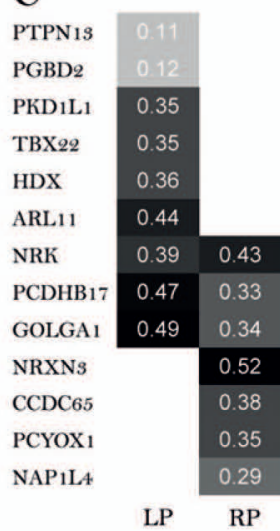

Figure 2. Intratumoural heterogeneity in familial PCC. (A) Photograph of tumour specimen following surgical excision. Dotted lines demarcate left pole (LP) and right pole (RP) regions sampled for multi-region analysis. (B) High-resolution copy-number analysis showing copy-number changes detected in left and right poles. Significant differences between samples are indicated by arrows including loss of chromosome 21 in the left pole and a small region of gain on chromosome 5 in the right pole. (C) Heatmap representation of the variant allele frequency for all somatic subtle mutations detected between the two poles. The grey-scale intensity and numerical value indicate the proportion of reads representing the variant allele as a proportion of the total reads for that base.

TMEM127 germline mutation and a somatic VHL missense mutation that were not detected in the exome-seq data due to low or no read coverage. Two intragenic structural alterations involving NF1 could also be identified through analysis of RNA-seq and copy-number data (Supplementary Figures 5 and 6). These examples demonstrate the importance of integrating multiple data types for improving mutation detection sensitivity and the identification of larger structural alterations.

The integration of multiple data types provided cumulative evidence for the potential functional importance of somatic mutations in known and putative cancer genes (Figure 3B). As expected, many known tumour-suppressor genes frequently harboured protein-truncating mutations, with concordant copy-number loss and high VAF $(>0.7)$, indicating loss of the wild-type copy (eg NF1). Some known mutated genes also exhibited comparatively low gene expression in the same tumours, which may be attributed to either genetic (ie copy-number loss) or epigenetic causes.

We identified somatic mutations in tumour-suppressor genes associated with maintenance of genome stability and DNA repair. A nonsense mutation in cohesin complex subunit STAG2 was identified in the sample displaying chromothripsis. A splice acceptor site mutation was identified in the Fanconi anaemia complementation group gene $P A L B 2$. Importantly, $P A L B 2$ expression in the mutated sample was down-regulated in the absence of additional copy-number loss, suggesting epigenetic silencing of the wild-type allele. A TP53 nonsense mutation was identified in one case with a corresponding copy-number loss at the same locus.

Another important gene group included genes associated with chromatin regulation. Two tumours had protein-truncating mutations in the H3.3 histone chaperone $A T R X$. Similarly, a missense mutation was found in the histone chaperones HIRA and NAPILA. Predominantly protein-truncating mutations and intragenic fusion break points could be identified in histone modifiers (CREBBP, EP300, WHSC1L1). Further mutations could be found in SWI/SNF chromatin remodellers (SMARCA4, SMARCA5, SMARCAL) and the chromodomain helicase $\mathrm{CHD} 4$.

The interpretation of other somatic mutations was made difficult by the low frequency of mutations and by the paucity of known genes affected. Despite this, we were able to group some genes into biological processes or pathways likely disrupted in PCC/PGL (Table 1). We identified FMRl mutations in two tumours. FMRl is associated with fragile-X mental retardation and regulates synaptic plasticity through RNA transport and negative regulation of protein translation [60]. Other mutated genes important for neural function included developmental transcription factors, genes associated with neuritogenesis, Rac/Rho signalling, and cytoskeletal remodelling (eg microtubule and actin filaments). Mutations were also found in genes associated with the Golgi complex, intracellular transport, vesicle membrane docking, and RAB pathway-associated genes. Few mutations were found in canonical signal transduction pathways; however, we noted a deleterious mutation in STAT3 and concordant low gene-expression in the same sample. This would be consistent with STAT3 having a putative tumour-suppressor function in PCC/PGL, similar to that reported in models of thyroid and liver cancer $[61,62]$.

We carefully looked for evidence of oncogenic fusions and detected an in-frame fusion between $A L K$ and PLEKHA6 in one tumour. Despite validation of the fusion and overexpression of $A L K$ at the mRNA level, we were unable to detect ALK protein expression in the tumour extract (Supplementary Figure 7). Furthermore, we could not find evidence of ALK overexpression by immunohistochemistry in another 172 archival PCC/PGL samples (data not shown). 
A

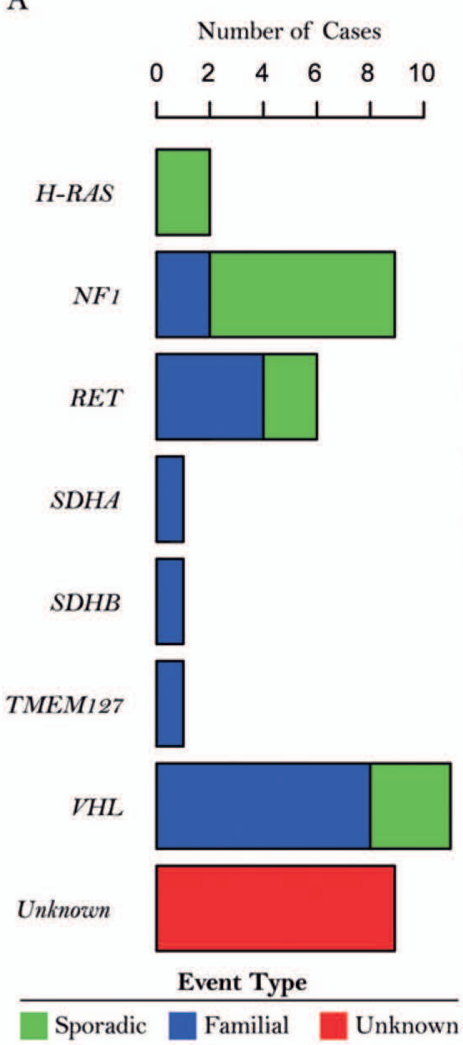

B

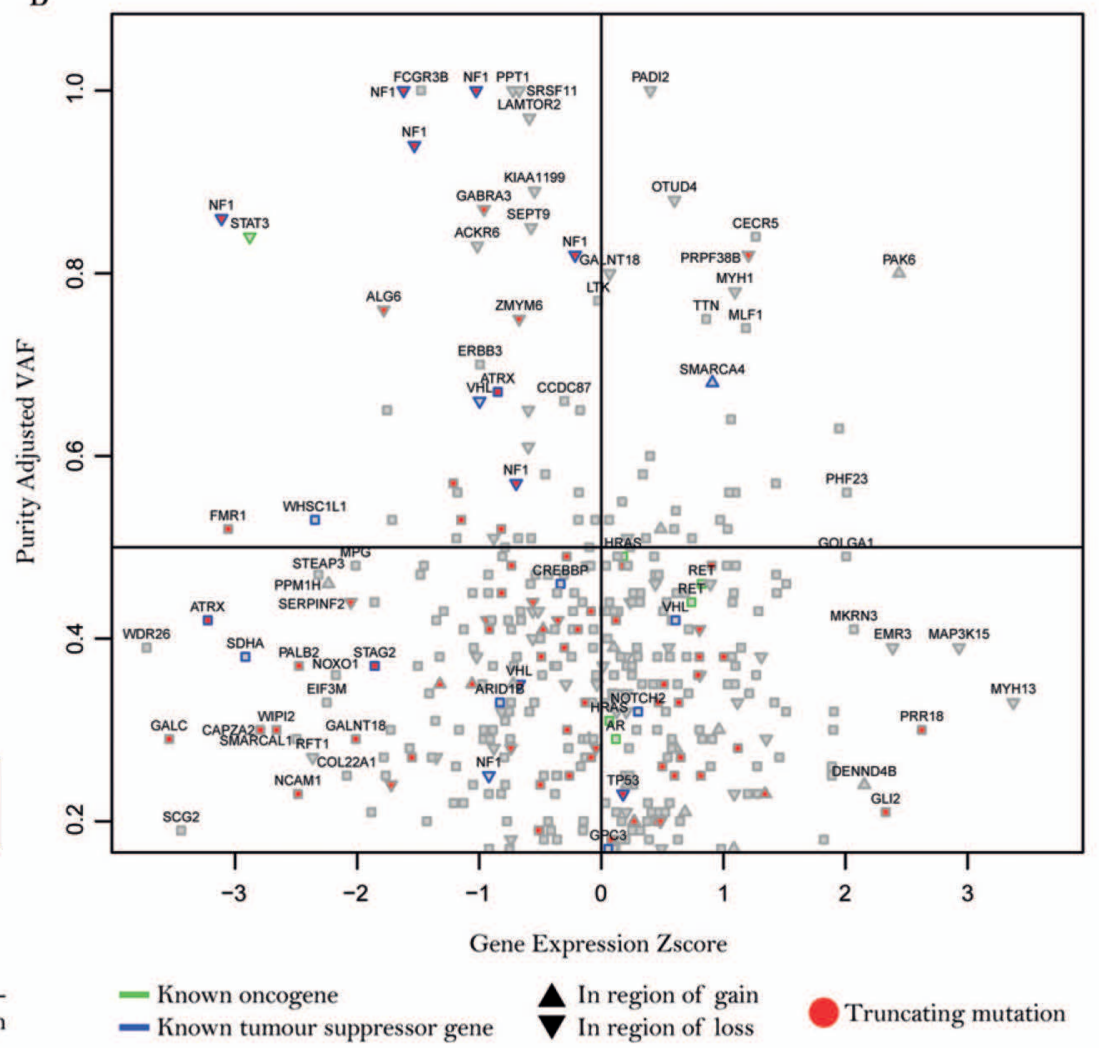

Figure 3. Known and putative driver genes detected in PCC/PGL. (A) Frequency of known PCC/PGL driver genes found to be mutated within the cohort. (B) Outlier analysis of somatically mutated genes. The $X$-axis represents deviation from the mean expression across the cohort as a $Z$-score $(Z$-score unit $=1$ standard deviation); the $Y$-axis represents the proportion of reads representing the mutation relative to the number of reads for the base, also known as the variant allele frequency (VAF). The VAF values have been adjusted to account for variation in tumour purity using either ASCAT purity estimation or purity estimation from the highest VAF value for the variant in that sample if this value was higher than that predicted by ASCAT.

\section{Gene-expression subtyping and data integration}

To correlate gene-expression subtypes with mutation and copy-number data, we undertook a comprehensive gene-expression analysis, utilizing both publicly available microarray datasets and RNA-seq data from our own cohort. We firstly used a compendium of three published Affymetrix microarray datasets $[6,7,21]$ to perform a consensus-clustering analysis that identified six robust gene-expression subtypes (Figures $4 \mathrm{~A}$ and 4B). Further segregation into seven or more subtypes resulted in the splintering of minor groups that represented less than $2 \%$ of the samples. Using the available genetic annotation, we could identify the expected pseudo-hypoxia or 'cluster 1' subtypes associated with mutations in $S D H$ subunits and $V H L$, respectively. Three other subtypes (referred to here as RTK1-3) included samples harbouring mutations in RET, NFI, and TMEM127, previously referred to as 'cluster 2'. A sixth subtype that shared a closer resemblance to RTK subtypes was composed of samples primarily lacking mutations in known PCC/PGL genes.

Using the microarray compendium and six-class annotation, we next trained a cross-platform classifier to classify samples from other gene-expression datasets. We applied the classifier to a microarray dataset published by López-Jiménez et al [63] demonstrating a general concordance between subtype, genotype, and gene-expression profile (Figure 4C). Importantly, the López-Jiménez dataset included four MAX mutant cases of which three were classified to the subtype lacking other PCC/PGL gene mutations. We subsequently referred to this group as the 'MAX-like' subtype.

Applying the cross-platform classifier to our RNA-seq dataset again demonstrated high concordance between the subtype and genotype of the tumours (Figure 4D). All tumours classified to the MAX-like subtype lacked mutations in known PCC/PGL driver genes. The uniqueness of the MAX-like tumours was further exemplified by the uncharacteristic copy-number alterations in this group (Supplementary Figure 4). The MAX-like group included one case with $3 p$ and chromosome 14 loss (VPH19), a pattern commonly associated with VHL mutant tumours; the case with chromothripsis of chromosome 11 (VCB02); a tumour with no detectable copy-number changes (VPH17); and two tumours with aneuploidy involving numerous chromosomal duplications with deletion of chromosome 11 in one tumour (VCB05, VPH15). We did not find any recurrently mutated genes amongst the MAX-like tumours. Interestingly, we observed low outlier $M A X$ expression $(Z$-score $<1.5)$ in three MAX-like cases, which corresponded to chromosome 14 loss in one case, although 


\begin{tabular}{|c|c|c|}
\hline Gene category & Biological process/pathway & Genes* \\
\hline Genome maintenance & $\begin{array}{l}\text { DNA repair } \\
\text { Sister chromatid cohesion } \\
\text { p53 pathway } \\
\text { Telomere maintenance }\end{array}$ & 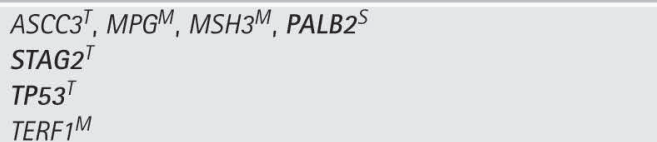 \\
\hline Epigenetic control & $\begin{array}{l}\text { SWI/SNF chromatin remodellers } \\
\text { Histone modifiers } \\
\text { Chromodomain helicases/NuRD complex } \\
\text { Histone chaperones }\end{array}$ & 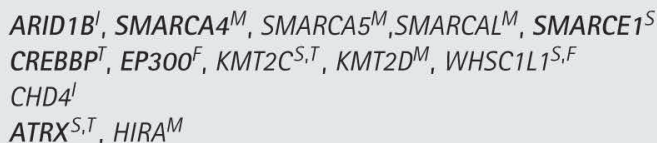 \\
\hline Transcription & $\begin{array}{l}\text { RNA processing } \\
\text { RNA transcriptional elongation } \\
\text { MicroRNA processing }\end{array}$ & 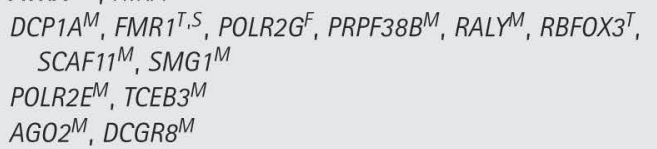 \\
\hline Protein metabolism & $\begin{array}{l}\text { Protein translation } \\
\text { Golgi and post-translational glycosylation of proteins } \\
\text { Intracellular transport and vesicle membrane docking } \\
\text { RAB and effector proteins }\end{array}$ & 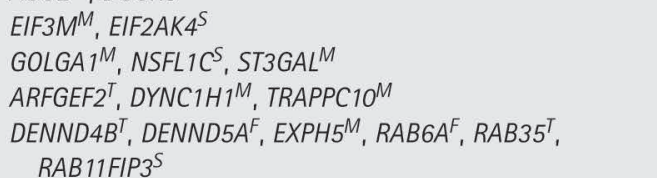 \\
\hline Cytoskeletal remodelling & $\begin{array}{l}\text { Rac/Rho pathway } \\
\text { Neurite outgrowth } \\
\text { Microtubule } \\
\text { Actin and spectrin } \\
\text { Synaptic membrane structure } \\
\text { lon channels }\end{array}$ & 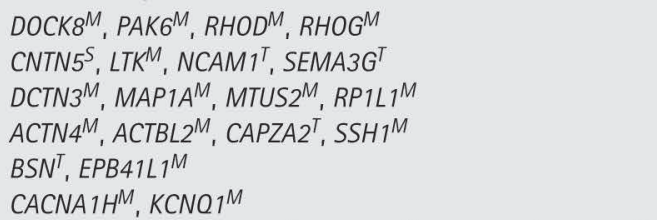 \\
\hline Cell adhesion & Cell adhesion & $\begin{array}{l}\text { BARX2 }{ }^{F}, C D 44^{F}, C O L 17 A 1^{\top}, C^{\prime} C_{N N A 3^{M}}, C T N N D 1^{F}, H M C N 1^{M}, \\
\text { NRXN }\end{array}$ \\
\hline Development & $\begin{array}{l}\text { Neuroblast differentiation } \\
\text { Developmental transcription factors }\end{array}$ & $\begin{array}{l}\text { AHNAKM }{ }^{M}, A H N A K 2^{M} \\
\text { ASCL1 } 1^{\prime}, P O U 6 F 2^{\prime}, S I X 2^{T}\end{array}$ \\
\hline Signalling & $\begin{array}{l}\text { STAT3 signalling } \\
\text { Cell surface receptors } \\
\text { Hedgehog signalling } \\
\text { MAPK pathway }\end{array}$ & $\begin{array}{l}\text { PIAS3 }^{S}, \text { STAT }^{S} \\
\text { ERBB3 } \\
\text { GLI2 }, 2^{S}, \text { STKR } 166^{M} \\
\text { LAMTOR2 } \\
\text { LMAP3K1 } 15^{M}\end{array}$ \\
\hline $\begin{array}{l}\text { Apoptosis } \\
\text { Cell cycle }\end{array}$ & $\begin{array}{l}\text { Apoptosis } \\
\text { Cell cycle }\end{array}$ & $\begin{array}{l}\text { AIFM } 3^{M}, \text { BIRC } 6^{M} \\
\text { CDC25AM SEPTIM }\end{array}$ \\
\hline
\end{tabular}

$\mathrm{M}=$ missense $; \mathrm{T}=$ truncating; $\mathrm{S}=$ splice region; $\mathrm{I}=$ in-frame $\operatorname{InDe} \mid ; \mathrm{F}=$ fusion/gene breakage.

"Known cancer genes are indicated in bold.

chromosome 14 loss did not correlate with low MAX expression in other tumours (Supplementary Figure 8). Single-copy gain of $M Y C$ was also observed in two MAX-like cases but this did not correlate with gain of $M Y C$ gene expression.

\section{Discussion}

We describe here the first comprehensive genomic analysis of a broad spectrum of genotypes within PCC/PGL tumours by MPS. All tumours had a relatively low mutation frequency similar to other neuroendocrine cancers of the pancreas and small intestine, medullary thyroid tumours, and neuroblastoma [64-66]. Despite the relatively low frequency of mutations in individual tumours, we identified a number of somatic changes that are likely contributing to disease development. A proportion of these affected genes are known to be tumour-suppressor genes in other cancer types. Our observations are therefore consistent with PCC/PGL being driven primarily by impaired tumour-suppressor gene function.

Multi-region sampling from a benign familial tumour provided evidence for intratumoural heterogeneity in PCC. All tumour cells ostensibly had a common ancestor as they shared the majority of copy-number alterations and a proportion of subtle mutations. Shared copy-number alterations between the tumour regions, including the $1 \mathrm{p}$ and $3 \mathrm{q}$ loss, indicate that these alterations are important early founder events, which is consistent with observations from other studies that have compared matched primary and metastatic tumours $[33,35]$. It is less clear what influence the subtle mutations had on tumour cell fitness, owing to our limited knowledge of the affected genes in the context of PCC. However, based on the detection of 'private' high VAF mutations and copy-number events, tumour evolution was likely an ongoing process. Evidence for intratumoural heterogeneity (ITH) in PCC/PGL could also be found in other tumours based on low VAF somatic variants and sub-copy-number loss detected by SNP-array analysis in some cases indicating the presence of sub-clones within tumours (Supplementary Figure 9). Although mutation burden and the general degree of ITH in PCC is lower than in other more aggressive tumour types, ITH in PCC appears to be higher than recently described in familial renal cell carcinomas [67]. Multi-region type analysis of additional PCC tumours will be required to validate and generalize our findings. The degree of ITH in PCC/PGL may have 

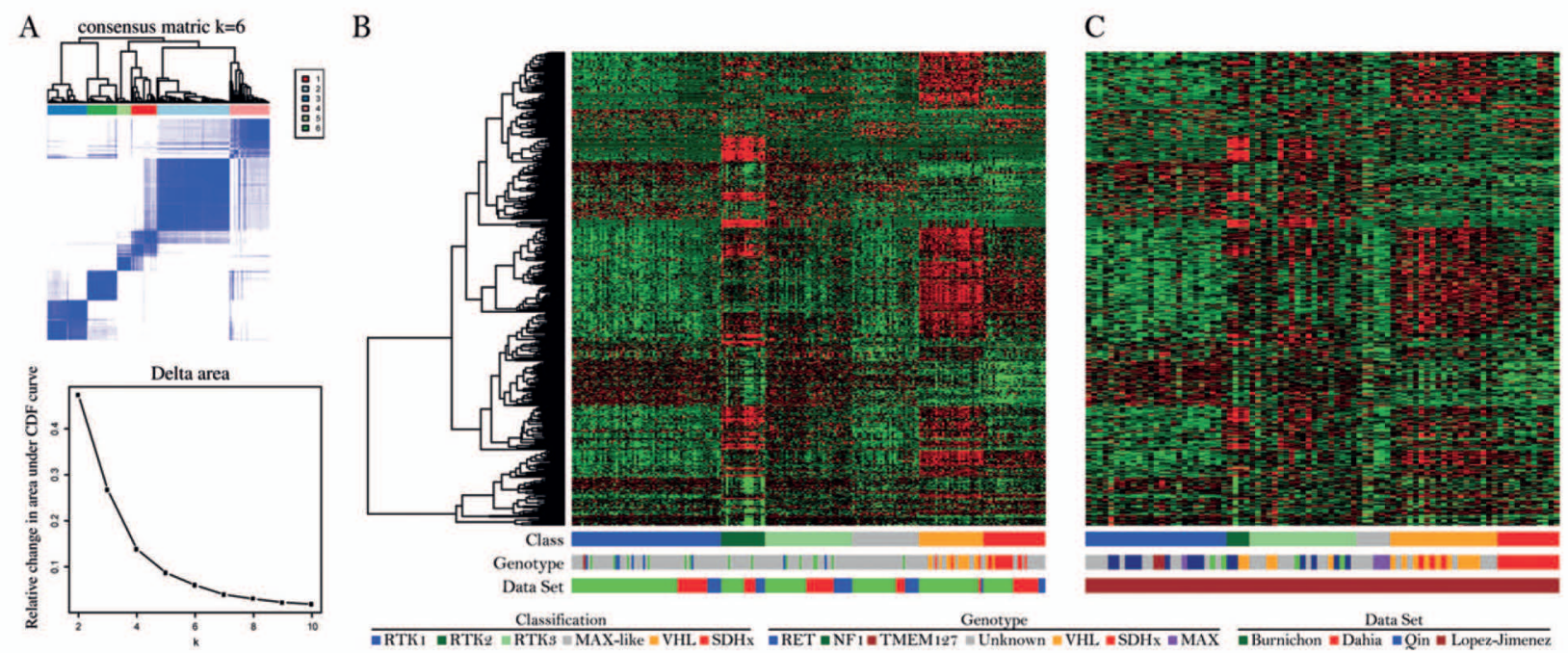

$\mathrm{D}$
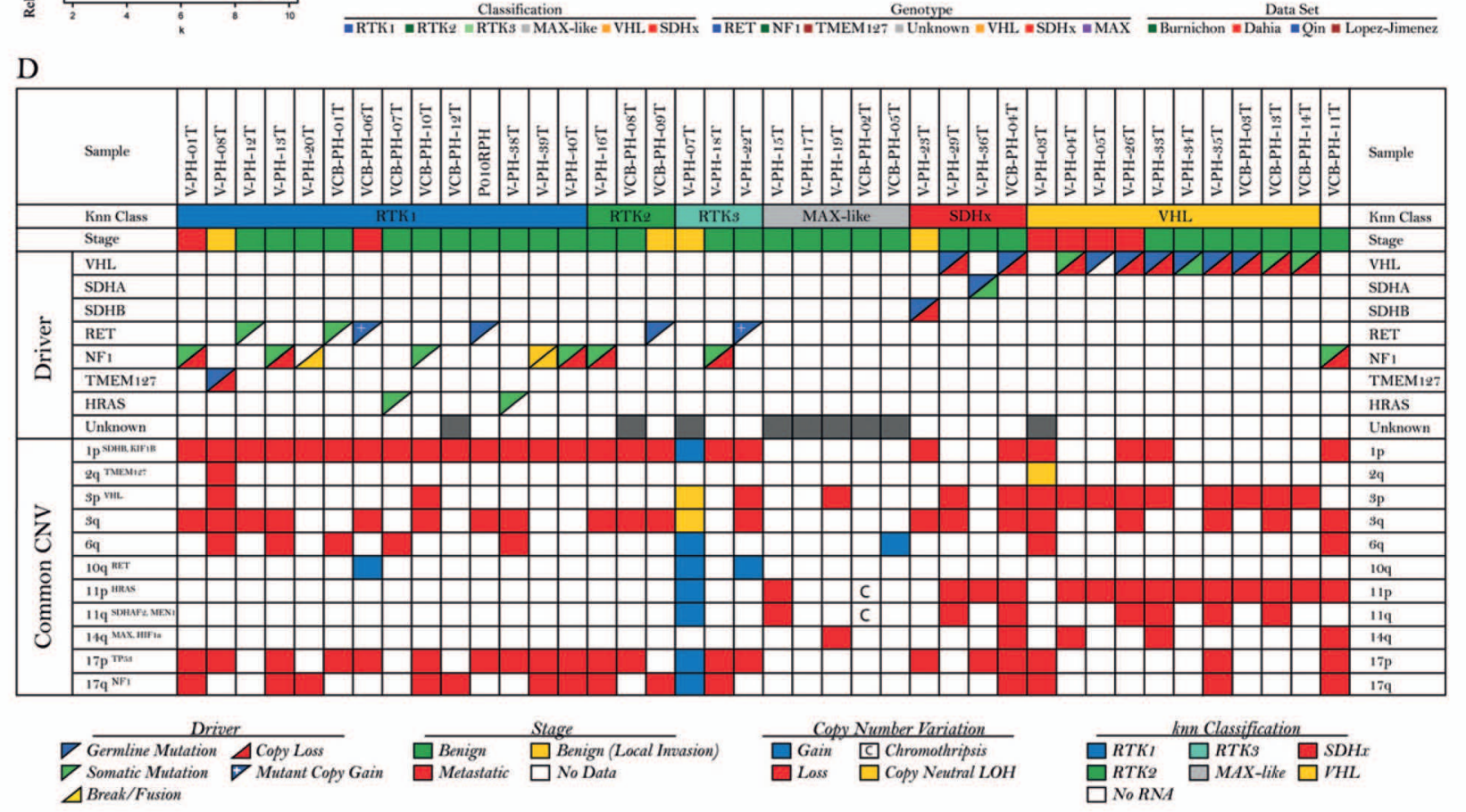

Figure 4. Consensus clustering by gene-expression profiling, subtyping, and data integration. (A) Top: consensus matrix showing cluster stability using a compendium of three published PCC/PGL microarray datasets. The arrays in the dataset are represented on both the $X$ and the $Y$-axis; the intensity of colour indicates the proportion of iterations in which those samples clustered together. Bottom: delta area plot showing the change in area under the cumulative distribution function curve illustrating the relative increase in stability gained by increasing the number of clusters $(k)$ - lower change in area under the curve corresponds to increased stability. (B) Gene-expression heatmap for samples used in the microarray compendium. Samples are ordered by the six identified subtypes in the $X$-axis. Genes are clustered by average linkage hierarchical clustering using a Pearson correlation in the $Y$-axis. Class annotation, genotype, and dataset origin are shown in bars below the heatmap. (C) Gene-expression heatmap for independent PCC/PGL microarray data test set. Genes and samples are ordered as in panel B. (D) Integration of expression subtype, identified PCC/PGL driver mutation, and common regions of copy-number alteration found in PCC/PGL.

implications as to whether metastatic potential can be predicted by genetic analysis if the tumour genome does not remain static.

Large and recurrent structural alterations are a common and clearly important feature of PCC/PGL genomes. It therefore stands to reason that disruption of genes important for maintenance of genome integrity could be pivotal drivers of tumourigenesis. Intriguingly, we identified a $S T A G 2$ mutation in a tumour displaying evidence of chromothripsis. STAG2 is a core component of a cohesin complex that regulates sister chromatid separation [68,69]. STAG2 mutations have been associated with aneuploidy in cancer cells [70]. To our knowledge, no prior association has been made between STAG2 mutations and chromothripsis. This would fit with the model of chromothripsis involving sequential processes of micronucleus formation resulting from defective sister chromatid separation followed by delayed DNA replication and premature chromosomal condensation, which results in chromosomal shattering $[71,72]$. The relative importance of chromothripsis in the PCC/PGL tumour sequenced is highlighted by the fact that few other genetic events could be identified and that the tumour did not harbour any known driver mutation. 
An altered epigenetic state and somatic mutations within chromatin regulators are frequently observed in a broad range of cancer types [73]. Disruption of histone modifiers and remodellers is also clearly important in PCC/PGL. These observations are consistent with the dysregulation of DNA and histone modifiers by succinate-dependent inhibition in SDH-mutant PCC/PGL tumours $[74,75]$. We identified a recurrence of protein truncating ATRX mutations in PCC/PGL. Similar observations have been made in other neuroendocrine cancer types such as pancreatic neuroendocrine tumours and neuroblastoma $[64,76]$. ATRX and its binding partner DAXX form part of a protein complex required for insertion of the histone variant $\mathrm{H} 3.3$ at telomeres. Mutations in ATRX, DAXX, and $H 3 F 3 A$ (H3.3) have been associated with alternative lengthening of telomeres (ALT) and higher levels of genomic instability [77-79]. Furthermore, ATRX loss is known to impair neuritogenesis through dysregulation of the Rac/Rho signalling pathway [80], which is concordant with mutations identified in other genes of related function.

Despite the use of exome sequencing, we did not resolve the driver mutation in a significant fraction of cases. More than half of these 'unknown' cases belong to a gene-expression subtype resembling MAX mutant PCC/PGL. The existence of a distinct gene-expression subtype involving $M A X$ mutant tumours is supported by a recent study by Qin et al [8]. MAX mutant tumours display intermediate levels of PNMT, corresponding with impaired epinephrine secretion [27]. Similarly, the MAX-like tumours have intermediate PNMT expression and are distinct from other tumours based on differential expression of other previously described PCC genes (Supplementary Figure 10). MAX mutations evidently account for only a small fraction of tumours in the MAX-like group. Germline and somatic MAX mutations account for $1.12 \%$ and $1.65 \%$ of familial and sporadic cases, respectively [27], whilst we estimate that the MAX-like subtype constitutes $\sim 10 \%$ or more of all PCC/PGL tumours. Our observation of low $M A X$ expression in some MAX-like tumours may suggest dysregulation of upstream factors regulating $M A X$ gene expression. Resolving any new putative driver genes in the MAX-like group will require a concentrated genomic analysis involving a larger number of samples from this group.

We have provided a first insight into co-operative genetic lesions in PCC/PGL tumours. DNA sequencing of a larger sample series will now be required to validate the findings of this study and to answer clinically relevant questions such as to genetic differences between benign and metastatic disease. Whole genome sequencing will be necessary to resolve gene drivers in some cases by enabling analysis of non-coding regions. Further investigation is needed to understand the observed differences between some gene-expression subtypes, including the underlying molecular differences between RTK groups. Importantly, in this study we have identified mutations in genes that may represent new therapeutic opportunities in treating this disease. For example, inactivating mutations in genome maintenance and DNA repair pathway genes such as STAG2 and PALB2 have been shown to render cancer cells sensitive to PARP inhibition $[81,82]$. The identification of such mutations in metastatic PCC/PGL may provide new therapeutic possibilities for PCC/PGL patients.

\section{Acknowledgments}

We would like to thank the patients and clinicians who provided materials and information for the study. This study was supported by the Victorian Cancer Agency through a translational research grant to RH. RC-B, DB, and $\mathrm{BR}$ received support from the Hillcrest Foundation and from the Pheo-Para Alliance.

\section{Author contribution statement}

$\mathrm{AF}, \mathrm{DB}, \mathrm{RCB}, \mathrm{BR}, \mathrm{RH}$, and RT conceived the study. $\mathrm{AF}, \mathrm{KW}, \mathrm{AH}$, and RT performed the experiments. DB, $\mathrm{RCB}, \mathrm{BR}, \mathrm{AT}$, and PJ provided material support. AF, JL, and JG performed bioinformatic analysis of the data. SF and $A G$ provided pathological interpretation. RCB, GM, and RH contributed to clinical interpretation. AF and RT wrote the manuscript. All authors contributed to revision of the manuscript and approved the final version.

\section{References}

1. Lenders JWM, Eisenhofer G, Mannelli M, et al. Phaeochromocytoma. Lancet 2005; 366: 665-675.

2. Karagiannis A, Mikhailidis DP, Athyros VG, et al. Pheochromocytoma: an update on genetics and management. Endocr Relat Cancer 2007; 14: 935-956.

3. Welander J, Söderkvist P, Gimm O. Genetics and clinical characteristics of hereditary pheochromocytomas and paragangliomas. Endocr Relat Cancer 2011; 18: R253-R276.

4. Ayala-Ramirez M, Feng L, Johnson MM, et al. Clinical risk factors for malignancy and overall survival in patients with pheochromocytomas and sympathetic paragangliomas: primary tumor size and primary tumor location as prognostic indicators. J Clin Endocrinol Metab 2011; 96: 717-725.

5. Dahia PLM. Pheochromocytoma and paraganglioma pathogenesis: learning from genetic heterogeneity. Nature Rev Cancer 2014; 14: 108-119.

6. Burnichon N, Vescovo L, Amar L, et al. Integrative genomic analysis reveals somatic mutations in pheochromocytoma and paraganglioma. Hum Mol Genet 2011; 20: 3974-3985.

7. Dahia PLM, Ross KN, Wright ME, et al. A HIF1 $\alpha$ regulatory loop links hypoxia and mitochondrial signals in pheochromocytomas. PLoS Genet 2005; 1: 72-80.

8. Qin N, de Cubas AA, Garcia-Martin R, et al. Opposing effects of HIF $1 \alpha$ and HIF $2 \alpha$ on chromaffin cell phenotypic features and tumor cell proliferation: insights from MYC-associated factor X. Int $J$ Cancer 2014; 135: 2054-2064.

9. Kaelin WG. The von Hippel-Lindau tumour suppressor protein: $\mathrm{O}_{2}$ sensing and cancer. Nature Rev Cancer 2008; 8: 865-873.

10. Ladroue C, Carcenac R, Leporrier M, et al. PHD2 mutation and congenital erythrocytosis with paraganglioma. N Engl J Med 2008; 359: 2685-2692. 
11. Astuti D, Latif F, Dallol A, et al. Gene mutations in the succinate dehydrogenase subunit SDHB cause susceptibility to familial pheochromocytoma and to familial paraganglioma. Am J Hum Genet 2001; 69: 49-54.

12. Baysal BE, Ferrell RE, Willett-Brozick JE, et al. Mutations in SDHD, a mitochondrial complex II gene, in hereditary paraganglioma. Science 2000; 287: 848-851.

13. Burnichon N, Brière J-J, Libé R, et al. SDHA is a tumor suppressor gene causing paraganglioma. Hum Mol Genet 2010; 19: 3011-3020.

14. Hao H-X, Khalimonchuk O, Schraders M, et al. SDH5, a gene required for flavination of succinate dehydrogenase, is mutated in paraganglioma. Science 2009; 325: 1139-1142.

15. Niemann S, Müller U. Mutations in SDHC cause autosomal dominant paraganglioma, type 3. Nature 2000; 26: 268-270.

16. Clark GR, Sciacovelli M, Gaude E, et al. Germline FH mutations presenting with pheochromocytoma. J Clin Endocrinol Metab 2014; 99: E2046-2050.

17. Selak MA, Armour SM, MacKenzie ED, et al. Succinate links TCA cycle dysfunction to oncogenesis by inhibiting HIF-alpha prolyl hydroxylase. Cancer Cell 2005; 7: 77-85.

18. Toledo RA, Qin Y, Srikantan S, et al. In vivo and in vitro oncogenic effects of HIF $2 A$ mutations in pheochromocytomas and paragangliomas. Endocr Relat Cancer 2013; 20: 349-359.

19. Mulligan LM, Kwok JB, Healey CS, et al. Germ-line mutations of the RET proto-oncogene in multiple endocrine neoplasia type $2 \mathrm{~A}$. Nature 1993; 363: 458-460.

20. Ferner RE. Neurofibromatosis 1. Eur J Hum Genet 2007; 15: 131-138.

21. Qin Y, Yao L, King EE, et al. Germline mutations in TMEM127 confer susceptibility to pheochromocytoma. Nature Genet 2010; 42: 229-233.

22. Comino-Méndez I, Gracia-Aznárez FJ, Schiavi F, et al. Exome sequencing identifies MAX mutations as a cause of hereditary pheochromocytoma. Nature Genet 2011; 43: 663-667.

23. Schlisio S, Kenchappa RS, Vredeveld LCW, et al. The kinesin $\mathrm{KIF} 1 \mathrm{~B} \beta$ acts downstream from $\mathrm{EgIN} 3$ to induce apoptosis and is a potential 1p36 tumor suppressor. Genes Dev 2008; 22: 884-893.

24. Yeh I-T, Lenci RE, Qin Y, et al. A germline mutation of the KIF1B $\beta$ gene on $1 \mathrm{p} 36$ in a family with neural and nonneural tumors. Hum Genet 2008; 124: 279-285.

25. Yoshimoto K, Iwahana H, Fukuda A, et al. ras mutations in endocrine tumors: mutation detection by polymerase chain reaction-single strand conformation polymorphism. Jpn J Cancer Res 1992; 83: 1057-1062.

26. Burnichon N, Buffet A, Parfait B, et al. Somatic NF1 inactivation is a frequent event in sporadic pheochromocytoma. Hum Mol Genet 2012; 21: 5397-5405.

27. Burnichon N, Cascon A, Schiavi F, et al. MAX mutations cause hereditary and sporadic pheochromocytoma and paraganglioma. Clin Cancer Res 2012; 18: 2828-2837.

28. Comino-Méndez I, de Cubas AA, Bernal C, et al. Tumoral EPASI (HIF2A) mutations explain sporadic pheochromocytoma and paraganglioma in the absence of erythrocytosis. Hum Mol Genet 2013; 22: 2169-2176.

29. Crona J, Delgado Verdugo A, Maharjan R, et al. Somatic mutations in $H$-RAS in sporadic pheochromocytoma and paraganglioma identified by exome sequencing. J Clin Endocrinol Metab 2013; 98: E1266-E1271.

30. Oudijk L, de Krijger RR, Rapa I, et al. H-RAS mutations are restricted to sporadic pheochromocytomas lacking specific clinical or pathological features: data from a multi-institutional series. J Clin Endocrinol Metab 2014; 99: E1376-E1380.

31. Welander J, Larsson C, Bäckdahl M, et al. Integrative genomics reveals frequent somatic NFI mutations in sporadic pheochromocytomas. Hum Mol Genet 2012; 21: 5406-5416.
32. Zhuang Z, Yang C, Lorenzo F, et al. Somatic HIF 2A gain-of-function mutations in paraganglioma with polycythemia. N Engl J Med 2012; 367: 922-930.

33. Dannenberg H, Speel EJ, Zhao J, et al. Losses of chromosomes $1 p$ and $3 q$ are early genetic events in the development of sporadic pheochromocytomas. Am J Pathol 2000; 157: 353-359.

34. Jarbo C, Buckley PG, Piotrowski A, et al. Detailed assessment of chromosome 22 aberrations in sporadic pheochromocytoma using array-CGH. Int J Cancer 2006; 118: 1159-1164.

35. Sandgren J, Diaz de Stahl T, Andersson R, et al. Recurrent genomic alterations in benign and malignant pheochromocytomas and paragangliomas revealed by whole-genome array comparative genomic hybridization analysis. Endocr Relat Cancer 2010; 17: 561-579.

36. van Nederveen FH, Korpershoek E, deLeeuw RJ, et al. Arraycomparative genomic hybridization in sporadic benign pheochromocytomas. Endocr Relat Cancer 2009; 16: 505-513.

37. Lawrence MS, Stojanov P, Mermel CH, et al. Discovery and saturation analysis of cancer genes across 21 tumour types. Nature 2014; 505: 495-501.

38. Alexandrov LB, Nik-Zainal S, Wedge DC, et al. Deciphering signatures of mutational processes operative in human cancer. Cell Rep 2013; 3: 246-259.

39. Gerlinger M, Rowan AJ, Horswell S, et al. Intratumor heterogeneity and branched evolution revealed by multiregion sequencing. $N$ Engl J Med 2012; 366: 883-892.

40. McInerney-Leo AM, Marshall MS, Gardiner B, et al. Whole exome sequencing is an efficient and sensitive method for detection of germline mutations in patients with phaeochromocytomas and paragangliomas. Clin Endocrinol 2014; 80: 25-33.

41. Li H, Durbin R. Fast and accurate short read alignment with Burrows-Wheeler transform. Bioinformatics 2009; 25: 1754-1760.

42. Trapnell C, Pachter L, Salzberg SL. TopHat: discovering splice junctions with RNA-Seq. Bioinformatics 2009; 25: 1105-1111.

43. McKenna A, Hanna M, Banks E, et al. The Genome Analysis Toolkit: a MapReduce framework for analyzing next-generation DNA sequencing data. Genome Res 2010; 20: 1297-1303.

44. Cibulskis K, Lawrence MS, Carter SL, et al. Sensitive detection of somatic point mutations in impure and heterogeneous cancer samples. Nature Biotechnol 2013; 31: 213-219.

45. Larson DE, Harris CC, Chen K, et al. SomaticSniper: identification of somatic point mutations in whole genome sequencing data. Bioinformatics 2012; 28: 311-317.

46. Koboldt DC, Chen K, Wylie T, et al. VarScan: variant detection in massively parallel sequencing of individual and pooled samples. Bioinformatics 2009; 25: 2283-2285.

47. Roth A, Ding J, Morin R, et al. JointSNVMix: a probabilistic model for accurate detection of somatic mutations in normal/tumour paired next-generation sequencing data. Bioinformatics 2012; 28: 907-913.

48. Van Loo P, Nordgard SH, Lingjærde OC, et al. Allele-specific copy number analysis of tumors. Proc Natl Acad Sci U S A 2010; 107: 16910-16915.

49. Amarasinghe $\mathrm{KC}, \mathrm{Li}$ J, Hunter SM, et al. Inferring copy number and genotype in tumour exome data. BMC Genomics 2014; 15: 732.

50. Heider A, Alt R. virtualArray: a R/bioconductor package to merge raw data from different microarray platforms. BMC Bioinformatics 2013; 14: 75 .

51. Wilkerson M, Waltman P. ConsensusClusterPlus: ConsensusClusterPlus. R package version 1.20.0, 2013.

52. Smyth GK. Limma: linear models for microarray data. In Bioinformatics and Computational Biology Solutions Using R and Bioconductor, Gentleman R, Carey V, Huber W, et al (eds). Springer: New York, 2005; 473.

53. Robinson MD, McCarthy DJ, Smyth GK. edgeR: a Bioconductor package for differential expression analysis of digital gene expression data. Bioinformatics 2010; 26: 139-140. 
54. McPherson A, Hormozdiari F, Zayed A, et al. deFuse: an algorithm for gene fusion discovery in tumor RNA-Seq data. PLoS Comput Biol 2011; 7: e1001138

55. Young RJ, Waldeck K, Martin C, et al. Loss of CDKN2A expression is a frequent event in primary invasive melanoma and correlates with sensitivity to the CDK4/6 inhibitor PD0332991 in melanoma cell lines. Pigment Cell Melanoma Res 2014; 27: 590-600.

56. Korbel JO, Campbell PJ. Criteria for inference of chromothripsis in cancer genomes. Cell 2013; 152: 1226-1236.

57. Frank-Raue K, Machens A, Scheuba C, et al. Difference in development of medullary thyroid carcinoma among carriers of RET mutations in codons 790 and 791. Clin Endocrinol 2008; 69: 259-263.

58. Vaclavikova E, Dvorakova S, Sykorova V, et al. RET mutation Tyr791Phe: the genetic cause of different diseases derived from neural crest. Endocrine 2009; 36: 419-424.

59. Erlic Z, Hoffmann MM, Sullivan M, et al. Pathogenicity of DNA variants and double mutations in multiple endocrine neoplasia type 2 and von Hippel-Lindau syndrome. J Clin Endocrinol Metab 2010; 95: 308-313.

60. Santoro MR, Bray SM, Warren ST. Molecular mechanisms of fragile X syndrome: a twenty-year perspective. Annu Rev Pathol 2012; 7: 219-245.

61. Schneller D, Machat G, Sousek A, et al. p19(ARF)/p14(ARF) controls oncogenic functions of signal transducer and activator of transcription 3 in hepatocellular carcinoma. Hepatology 2011; 54: 164-172.

62. Couto JP, Daly L, Almeida A, et al. STAT3 negatively regulates thyroid tumorigenesis. Proc Natl Acad Sci U S A 2012; 109: E2361-E2370.

63. López-Jiménez E, Gómez-López G, Leandro-García LJ, et al. Research resource: transcriptional profiling reveals different pseudohypoxic signatures in SDHB and VHL-related pheochromocytomas. Mol Endocrinol 2010; 24: 2382-2391.

64. Jiao Y, Shi C, Edil BH, et al. DAXX/ATRX, MEN1, and mTOR pathway genes are frequently altered in pancreatic neuroendocrine tumors. Science 2011; 331: 1199-1203.

65. Banck MS, Kanwar R, Kulkarni AA, et al. The genomic landscape of small intestine neuroendocrine tumors. J Clin Invest 2013; 123: 2502-2508.

66. Agrawal N, Jiao Y, Sausen M, et al. Exomic sequencing of medullary thyroid cancer reveals dominant and mutually exclusive oncogenic mutations in RET and RAS. J Clin Endocrinol Metab 2013; 98: E364-E369
67. Fisher R, Horswell S, Rowan A, et al. Development of synchronous VHL syndrome tumors reveals contingencies and constraints to tumor evolution. Genome Biol 2014; 15: 268.

68. Haering $\mathrm{CH}$, Farcas A-M, Arumugam $\mathrm{P}$, et al. The cohesin ring concatenates sister DNA molecules. Nature 2008; 454: 297-301.

69. Nasmyth K. Segregating sister genomes: the molecular biology of chromosome separation. Science 2002; 297: 559-565.

70. Solomon DA, Kim T, Diaz-Martinez LA, et al. Mutational inactivation of STAG2 causes aneuploidy in human cancer. Science 2011; 333: 1039-1043.

71. Crasta K, Ganem NJ, Dagher R, et al. DNA breaks and chromosome pulverization from errors in mitosis. Nature 2012; 482: 53-58.

72. Wyatt AW, Collins CC. In brief: chromothripsis and cancer. J Pathol 2013; 231: 1-3.

73. Shen H, Laird PW. Interplay between the cancer genome and epigenome. Cell 2013; 153: 38-55.

74. Letouzé E, Martinelli C, Loriot C, et al. SDH mutations establish a hypermethylator phenotype in paraganglioma. Cancer Cell 2013; 23: 739-752.

75. Xiao M, Yang H, Xu W, et al. Inhibition of $\alpha-K G$-dependent histone and DNA demethylases by fumarate and succinate that are accumulated in mutations of FH and SDH tumor suppressors. Genes Dev 2012; 26: 1326-1338.

76. Cheung N-KV, Zhang J, Lu C, et al. Association of age at diagnosis and genetic mutations in patients with neuroblastoma. J Am Med Assoc 2012; 307: 1062-1071.

77. Heaphy CM, de Wilde RF, Jiao Y, et al. Altered telomeres in tumors with ATRX and DAXX mutations. Science 2011; 333: 425.

78. Lovejoy CA, Li W, Reisenweber S, et al. Loss of ATRX, genome instability, and an altered DNA damage response are hallmarks of the alternative lengthening of telomeres pathway. PLoS Genet 2012; 8: e1002772.

79. Baumann C, Viveiros MM, De La Fuente R. Loss of maternal ATRX results in centromere instability and aneuploidy in the mammalian oocyte and pre-implantation embryo. PLoS Genet 2010; 6: e1001137.

80. Shioda N, Beppu H, Fukuda T, et al. Aberrant calcium/calmodulin-dependent protein kinase II (CaMKII) activity is associated with abnormal dendritic spine morphology in the ATRX mutant mouse brain. J Neurosci 2011; 31: 346-358.

81. Bailey ML, O'Neil NJ, van Pel DM, et al. Glioblastoma cells containing mutations in the cohesin component STAG2 are sensitive to PARP inhibition. Mol Cancer Ther 2014; 13: 724-732.

82. Buisson R, Dion-Côté A-M, Coulombe Y, et al. Cooperation of breast cancer proteins PALB2 and piccolo BRCA2 in stimulating homologous recombination. Nature Struct Mol Biol 2010; 17: 1247-1254.

\section{SUPPORTING INFORMATION ON THE INTERNET}

The following supporting information may be found in the online version of this article:

Figure S1. Scatter plot showing the number of somatic mutations found in coding regions versus the age of the patient at diagnosis.

Figure S2. Frequency of chromosomal aberrations.

Figure S3. Chromothripsis.

Figure S4. Copy-number profiles of MAX-like tumour class.

Figure S5. Segmental homozygous deletion of NF1 exons 47-50.

Figure S6. Genomic breakage leading to inactivation of NF1.

Figure S7. ALK fusion.

Figure S8. Corresponding copy-number changes and gene expression.

Figure S9. Heterogeneity of copy-number alterations.

Figure S10. Comparison of microarray and RNA-seq data for previously described subtype markers. 


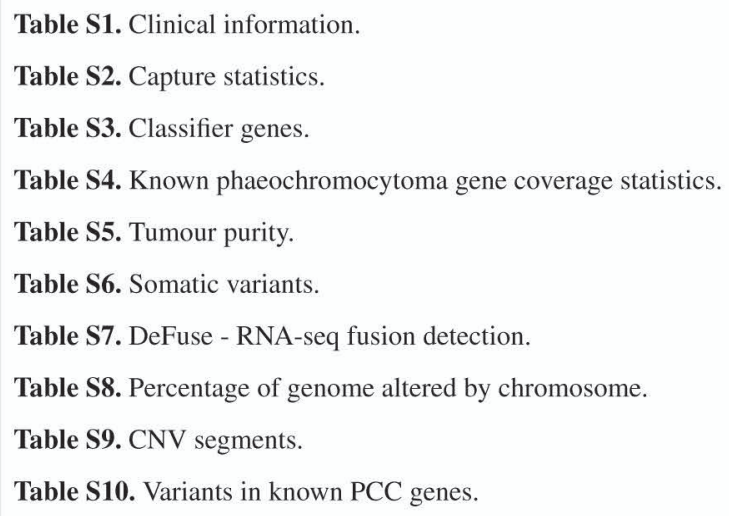

\section{Years ago in the Journal of Pathology...}

Histological studies and measurement of nucleic acid synthesis in rat mammary tumours induced by 9,10-dimethyl-1,2-benzanthracene (DMBA)

L. Stevens, Evelyn Stevens and A. R. Currie

Protection provided against carbon tetra-chloride and thioacetamide poisoning by strophanthin-G

J. D. Judah, K. Ahmed and A. E. M. McLean

The pathology of the early stages of experimental staphylococcal pyelonephritis in the guinea-pig and rat

W. D. Foster

To view these articles, and more, please visit: www.thejournalofpathology.com

Click 'ALL ISSUES (1892 - 2011)', to read articles going right back to Volume 1, Issue 1.

\section{The Journal of Pathology Understanding Disease}

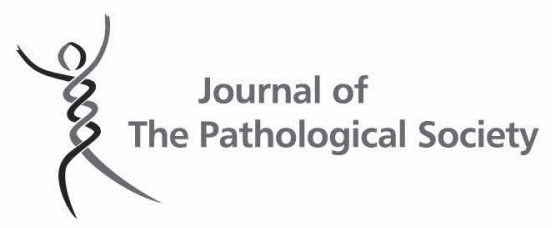




\section{A Diagnostic Gene-expression Assay for the Classification of PPGL}

\subsection{Chapter Preface}

As all cells in the body possess the same genetic information, the specialisation or differentiation of particular cell-types can be attributed to variation in the utilisation of this information. The subset of genes and the degree to which each is expressed is termed the cellular transcriptional program. During oncogenesis, cellular transcriptional programming is often perturbed, switching on or off cellular processes involved in cellular growth, proliferation, energy metabolism, motility, and viability (Hanahan \& Weinberg, 2011). The exact transcriptional pathways that become dysregulated are dependant both on the driver events that initiated oncogenesis and on the transcriptional program typical of the cell-type.

The advent of gene-expression profiling technologies such as microarray, and more recently RNA sequencing, have made it possible to examine the transcriptional activity of a cell or collection of cells. When applied to tumour cells these technologies provide insight into the disturbances to normal transcriptional programming that are driving tumour development. Taken as a whole, the expression profile of a tumour provides a signature for the underlying oncogenic events driving tumourigenesis. As such, these transcriptional signatures can be used to identify tumour subtypes that may have distinct biology in terms of initiation, progression, metastatic potential, and treatment response. This paradigm has been successfully applied to subtyping various cancer types including ovarian cancer (Tothill et al., 2008), triple negative breast cancer (Jezequel et al., 2015), and acute myeloid leukaemia (Mills et al., 2009).

As previously discussed, expression profiling experiments by Dahia et al. (2005) revealed two major subtypes of PPGL; one representing tumours with a dysregulation in HIF signalling, termed the pseudohypoxia tumours, and the other comprised of those with aberrant MAPK signalling. Following on from this work, Burnichon et al. (2011) used a larger sample set to further refine the subtypes, subdividing the pseudo-hypoxia group into those possessing a succinate dehydrogenase subunit mutation (designated $\mathrm{C} 1 \mathrm{~A}$ ) and those with a $V H L$ mutation (designated C1B), and subdividing the MAPK tumours into three subgroups (designated C2A, B, and C). As presented in Chapter Three, objective assessment of previously published data sets with a consensus clustering algorithm revealed six subtypes. Utilising the publically available annotation, it was determined that the subtypes are largely consistent with those observed by Burnichon et al. (Table 4.1), with $S D H x$ and $V H L$ type tumours represented in their own clusters, while tumours with MAPK signalling defects were spread across multiple clusters. A sixth cluster was found to lack mutations in any of the known PPGL driver genes. However, further 
analysis of publically available data demonstrated that $M A X$ mutant tumours fell into this class.

Table 4.1 Correlation matrix of Burnichon et al. (2011) clusters and consensus clustering subtypes

\begin{tabular}{|l|l|l|l|l|l|}
\hline & C1A & C1B & C2A & C2B & C2C \\
\hline MAX-Like & 0 & 0 & 0 & $\mathbf{1 9}$ & 0 \\
\hline RTK1 & 0 & 0 & $\mathbf{4 5}$ & 0 & 0 \\
\hline RTK2 & 0 & 0 & 0 & 0 & $\mathbf{1 0}$ \\
\hline RTK3 & 0 & 0 & $\mathbf{1 8}$ & 0 & 0 \\
\hline SDHx & $\mathbf{1 1}$ & $\mathbf{2}$ & 0 & 0 & 0 \\
\hline VHL & $\mathbf{2}$ & $\mathbf{2 4}$ & 0 & 0 & 0 \\
\hline
\end{tabular}

\subsection{Aims and Rationale}

Contrary to many cancer types, the significant contribution of inherited gene mutations to PPGL development indicates genetic testing as a valuable component of standard care following PPGL diagnosis. Historically, since genetic testing has been expensive as each PPGL gene had to be individually sequenced exon by exon, this lead to the development of decision-tree type approaches based on clinical information such as tumour location, catecholamine secretory profile, and immunohistochemistry (Benn et al., 2006; Jafri \& Maher, 2012). More recently, focussed massivelyparallel sequencing panels allowing all PPGL genes to be simultaneously sequenced have reduced both the cost and time burden of genetic testing, however clinical adoption is still in its infancy.

As the identification of a constitutional pathogenic mutation will inform genetic counselling of affected individuals and their relatives, it is of the utmost importance that genetic counsellors are confident in a molecular diagnosis before discussing the result with a patient. The strong subtype-to-genotype relationship seen in PPGL allows classification to provide orthogonal evidence for assessing the veracity of any mutations detected by clinical sequencing. For example, if an individual was found to be positive for a $V H L$ mutation and also possessed an expression profile typical of $V H L$ mutant tumours then a referring clinician could have additional confidence in the genetic diagnosis. In the setting of traditional Sanger dideoxynucleotide sequencing-based diagnostics, classification could also be used to prioritise the genes typical of the determined class, reducing the waiting time and associated anxiety a patient may experience, as well as potentially reducing costs to patients without adequate medical insurance.

While the need to prioritise genes is not relevant to high-throughput based approaches, these methods have distinct limitations of their own, including variants missed due to poor coverage and false positives caused by misalignment to the reference genome (Toledo \& Dahia, 2015). The scope of the data returned 
by massively parallel sequencing also exacerbates the 'variant of unknown significance' (VUS) conundrum, whereby a mutation is found in a known causative gene but the biological consequences are indeterminable with the available annotation. This scenario was exemplified in a recent publication describing a 23-year-old female with metastatic PPGL and no family history of disease (Russell et al., 2015). Diagnostic sequencing revealed a novel missense variant (c.361G $>C$ ) in the $V H L$ gene with unknown pathogenicity. The study employed various bioinformatics techniques such as examining evolutionary conservation and physiochemical properties of the affected residue. However, pathogenicity was ultimately decided upon detecting previously unrecognised features of von Hippel-Lindau disease in the mother of the patient, whom shared the variant. In scenarios such as this, classification would provide valuable insight into the biology of the tumour and the pathogenicity of a VUS.

In addition to mutation and INDEL events, large-scale chromosomal alterations can act as oncogenic drivers, as exemplified by the $N F 1$ breakage fusion events described in Chapter Three. These events are difficult to detect with targeted high-throughput or Sanger dideoxynucleotide sequencing alone. More recently, epigenetic inactivation of $S D H B$ and $S D H C$ has been described in patients with Carney-Stratakis syndrome-associated PGL (Haller et al., 2014), a mechanism of oncogenesis invisible to routine diagnostic sequencing. Although epigenetic silencing of $V H L$ has been implicated in the aetiology of renal cell carcinoma, with the exception of a recent study (Andreasson et al., 2013), there has been little evidence linking epigenetic silencing of VHL to PPGL development (Cascon et al., 2004; Welander et al., 2012). However, classification may assist in identifying tumours exhibiting $V H L$-like biology with no apparent mutation for further analysis.

Given the profound clinical significance of the identification of a hereditary cancer syndrome, the ability to validate molecular testing by an orthogonal approach would have significant benefit before major management decisions are made, even in cases in which a clearly pathogenic mutation is identified. However, the clinical application of PPGL gene-expression subtyping is currently hindered by the requirement for high quality RNA from fresh tissue to be compatible with most array or sequencing-based methods. The aim of this chapter was to develop a clinically robust assay to accurately classify both recently obtained and archival PPGL specimens into one of the six subtypes described in Chapter Three. And in the process of doing so, further elucidate the interactions and defining features of the PPGL subtypes. 


\subsection{The Nanostring Platform}

Standard practice for diagnostic pathology laboratories involves the fixation of surgical specimens in 10\% neutral buffered formalin, while this process is integral to preservation of cellular morphology and antigen retrieval for diagnostic purposes, it is known to drastically reduce the quality of retrievable nucleic acids (Bussolati et al., 2015). As such, any method of gene-expression profiling that relies on the availability of full-length mRNA molecules is unsuitable for use with formalin-fixed specimens.

The Nanostring platform is a hybridisation-based assay in which probes are designed to be complementary to a region of the gene or transcript of interest. Each transcript is targeted by a pair of probes approximately 50 base pairs in length; the first probe, termed the reporter probe, is conjugated to a fluorescent reporter molecule while the second, termed the capture probe, is labelled with biotin. The reporter and capture probes are incubated with isolated total RNA whereupon they bind to the transcript molecules of interest. The hybridised probe and RNA complexes are then immobilised on a glass slide and counted using florescence detection. In this way the Nanostring platform provides a digital count for the absolute abundance of a transcript. As the assay utilises hybridisation and only targets around 100 nucleotides of the transcript, fragmentation of target RNA does not impede transcript detection making it ideal for use with fresh tissue and fixed archival samples.

\subsection{Gene Selection}

In Chapter Three the process for selecting genes to develop a microarray-to-RNA-seq cross platform classifier was described. As microarray and RNA-Seq are both essentially whole transcriptome technologies, it was acceptable to utilise a large set of genes for training the classifier. However, the cost of a Nanostring assay is dependent on the number of genes utilised, given that assay cost is a consideration in both diagnostic and research settings, a more succinct list of forty-seven genes was derived to ameliorate the per sample cost (see Methods 2.3.3.1.3 and Figure 4.1). Six genes with a low coefficient of variance across all subtypes were selected for use as normalisation controls. During probe design, two genes were found to share a high degree of homology and were collapsed into a single probe set yielding a final list of 52 genes (Table 4.2 and Appendices C-H).

\subsection{Validating the Nanostring Platform}

A three-part validation was performed in order to assess, firstly, how well the Nanostring assay recapitulated the expression values obtained from RNA-seq, secondly, how well expression values obtained from FFPE material reflected those of fresh frozen material, and finally, the robustness of the classification between platforms. For this purpose, the Nanostring assay was performed on a set of eleven 
FFPE samples for which RNA-seq data were available. For a subset of five samples, the Nanostring assay was also performed on matched fresh-frozen material. To ensure a robust comparison the same RNA used for RNA-seq library preparation was also used in the Nanostring assay. Haematoxylin and eosin guided macro-dissection was performed on FFPE sections prior to RNA isolation (see Methods 2.2.1.2). The Nanostring assay and data normalisation were performed as described in methods sections 2.2.6 and 2.3.3.3.

Linear regression analysis demonstrated a strong positive correlation (mean $\mathrm{R}^{2}: 0.75$; range: 0.69-0.8) between RNA-seq and Nanostring using RNA from fresh frozen material (Figure 4.2a). In samples with lower r-squared scores the largest residuals are predominantly contributed by probes that are underrepresented in the RNA-seq relative to the Nanostring. This could be explained by several technical factors. Firstly, the sample preparation method for RNA-seq relies on selection of messenger RNA molecules via the poly-A tail, if the RNA sample is partially degraded this will result in a reduction of read counts as the bulk of the gene body will be missing and as such under-represented. Secondly, RNAseq relies on stochastic sampling of a population of RNA molecules, which means a given transcript may be under-represented by virtue of sequence coverage. Finally, as most of the poorly performing genes have low expression on both platforms, the expression may be below the reliable threshold for RNA-seq. Linear regression analysis of the five matched FFPE and fresh frozen samples demonstrated high concordance with a mean r-squared of 0.87 (range: 0.71-0.94) (Figure 4.2b), indicating that the assay was performing similarly on both fresh frozen and fixed specimens.

Next, a support vector machine was trained using expression data for the 46 classifier genes in the microarray superset and tested on the Nanostring expression data. Of the eleven cases tested, nine were classified into the same subtype based on both RNA-seq and Nanostring data. A sample with no known mutation shifted from MAX-like to RTK3, and a sample with a VHL mutation shifted from the SDHx subgroup to the RTK2 subtype. This movement between subtypes is likely the effect of regional heterogeneity and sample purity and is discussed further below. 
A

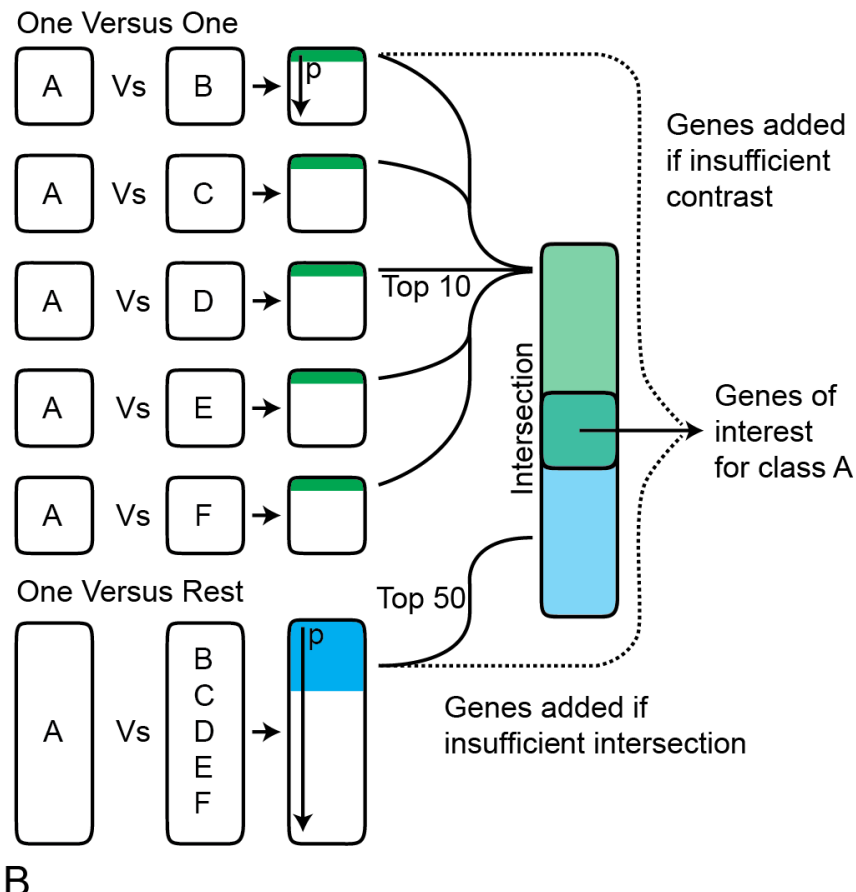

B
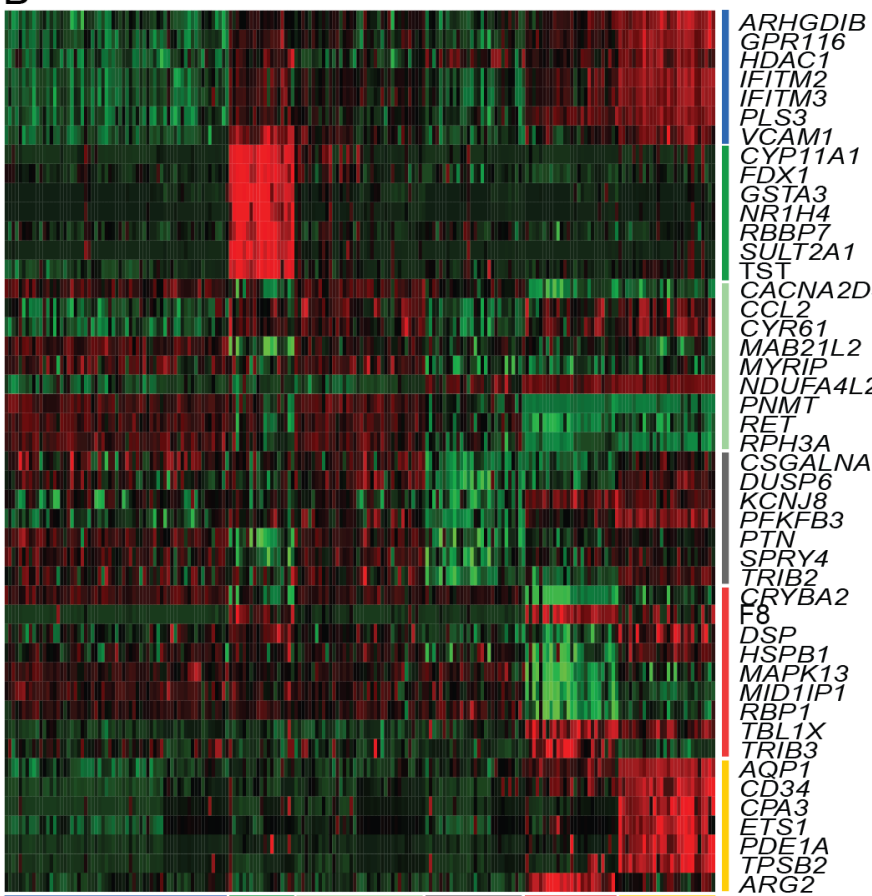

(

EYP11A1

NR1H4

SULT2A1

CACNA2D3

CCL 2

MAB21L2

MYRIP

NDUFA4L2

$P N M T$

RET

CSGALNACT1

DUSPG

PFKFB3

PTN
SPRY 4

TRIB2

CRYBA2

OSP

HSPB1

MIDIIP1

$R B P 1$

TBL1X

AQP 1

CPA3

PDE $1 A$

RTK1 RTK2 RTK3 MAX-Like SDHx VHL Samples

Figure 4.1 PPGL gene selection for Nanostring assay

(A) A combination of one-versus-one and one-versus-rest differential gene expression was used to select the genes most representative of each group. The top 10 genes, as ranked by $p$ value, from each one-versus-one comparison were combined then intersected with the top 50 genes from a one versus rest comparison. Where insufficient contrasting genes were selected, additional genes were added for the deficient class. (B) Heatmap representation of gene-expression in the microarray training data set for the 46 genes selected. Red indicates overexpression, and green indicates underexpression relative to mean expression for the gene across the cohort. Genes and samples are ordered by subtype as indicated by coloured bars. 
Table 4.2 Classifier genes

\begin{tabular}{|c|c|c|}
\hline Class & HGNC Symbol & Gene Description \\
\hline \multirow{7}{*}{ RTK1 } & PLS3 & Plastin 3 \\
\hline & IFITM2 & Interferon induced transmembrane protein 2 \\
\hline & IFITM3 & Interferon induced transmembrane protein 3 \\
\hline & ARHGDIB & Rho GDP dissociation inhibitor (GDI) beta \\
\hline & GPR116 & G protein-coupled receptor 116 \\
\hline & HDAC1 & Histone deacetylase 1 \\
\hline & VCAM1 & Vascular Cell Adhesion Molecule 1 \\
\hline \multirow{7}{*}{ RTK2 } & GSTA3 & Glutathione s-transferase alpha 3 \\
\hline & SULT2A1 & Sulfotransferase family, cytosolic, 2A, DHEA-preferring, member 1 \\
\hline & NR1H4 & Nuclear receptor subfamily 1 , group $H$, member 4 \\
\hline & TST & Thiosulfate sulfurtransferase (rhodanese) \\
\hline & CYP11A1 & Cytochrome P450, family 11 , subfamily A, polypeptide 1 \\
\hline & $R B B P 7$ & Retinoblastoma binding protein 7 \\
\hline & FDX1 & Ferredoxin 1 \\
\hline \multirow{9}{*}{ RTK3 } & PNMT & Phenylethanolamine $n$-methyltransferase \\
\hline & NDUFA4L2 & NADH dehydrogenase (ubiquinone) 1 alpha subcomplex, 4-like 2 \\
\hline & RPH3A & Rabphilin 3A homolog (mouse) \\
\hline & MYRIP & Myosin VIIA and Rab interacting protein \\
\hline & CACNA2D3 & Calcium channel, voltage-dependent, alpha $2 /$ delta subunit 3 \\
\hline & RET & Ret proto-oncogene \\
\hline & MAB21L2 & Mab-21-like 2 (C. Elegans) \\
\hline & CYR61 & Cysteine-rich, angiogenic inducer, 61 \\
\hline & CCL2 & Chemokine ( $\mathrm{C}-\mathrm{C}$ motif) ligand 2 \\
\hline \multirow{7}{*}{ MAX-Like } & TRIB2 & Tribbles homolog 2 (Drosophila) \\
\hline & DUSP6 & Dual specificity phosphatase 6 \\
\hline & SPRY4 & Sprouty homolog 4 (Drosophila) \\
\hline & CSGALNACT1 & Chondroitin sulfate $\mathrm{N}$-acetylgalactosaminyltransferase 1 \\
\hline & PFKFB3 & 6-phosphofructo-2-kinase/fructose-2,6-biphosphatase 3 \\
\hline & PTN & Pleiotrophin \\
\hline & KCNJ8 & Potassium inwardly-rectifying channel, subfamily J, member 8 \\
\hline \multirow{6}{*}{ VHL } & TPSAB1 & Tryptase alpha/beta 1 \\
\hline & CPA3 & Carboxypeptidase A3 (mast cell) \\
\hline & PDE1A & Phosphodiesterase $1 \mathrm{~A}$, calmodulin-dependent \\
\hline & ETS1 & V-ets erythroblastosis virus E26 oncogene homolog 1 (avian) \\
\hline & CD34 & CD34 molecule \\
\hline & $A Q P 1$ & Aquaporin 1 (Colton blood group) \\
\hline \multirow{10}{*}{ SDHx } & ARG2 & Arginase 2 \\
\hline & $R B P 1$ & Retinol binding protein 1 , cellular \\
\hline & MIDIIP1 & MID1 interacting protein 1 \\
\hline & MAPK13 & Mitogen-activated protein kinase 13 \\
\hline & CRYBA2 & Crystallin, beta $\mathrm{A} 2$ \\
\hline & TBLIX & Transducin (beta)-like 1X-linked \\
\hline & $D S P$ & Desmoplakin \\
\hline & $T R I B 3$ & Tribbles homolog 3 (Drosophila) \\
\hline & HSPB1 & Heat shock $27 \mathrm{kda}$ protein 1 \\
\hline & F8 & Coagulation factor VIII, procoagulant component \\
\hline \multirow{6}{*}{ Controls } & VDAC3 & Voltage-dependent anion channel 3 \\
\hline & SRSF3 & Serine/arginine-rich splicing factor 3 \\
\hline & ITM2B & Integral membrane protein 2B \\
\hline & MTCH1 & Mitochondrial carrier 1 \\
\hline & YWHAB & Tyr 3-monooxygenase/trp 5-monooxygenase activation protein beta \\
\hline & $A B C F 1$ & ATP-binding cassette, sub-family F (GCN20), member 1 \\
\hline
\end{tabular}


A

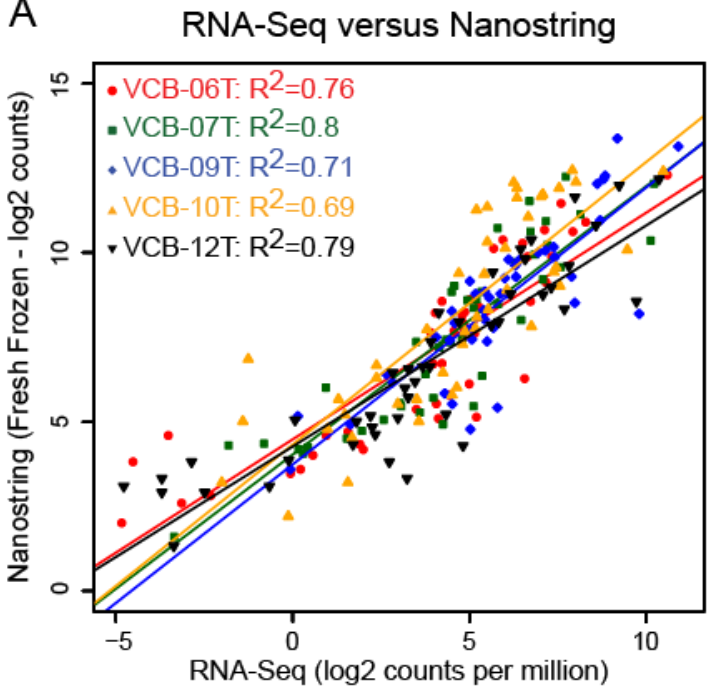

B Nanostring - Fresh Frozen versus FFPE

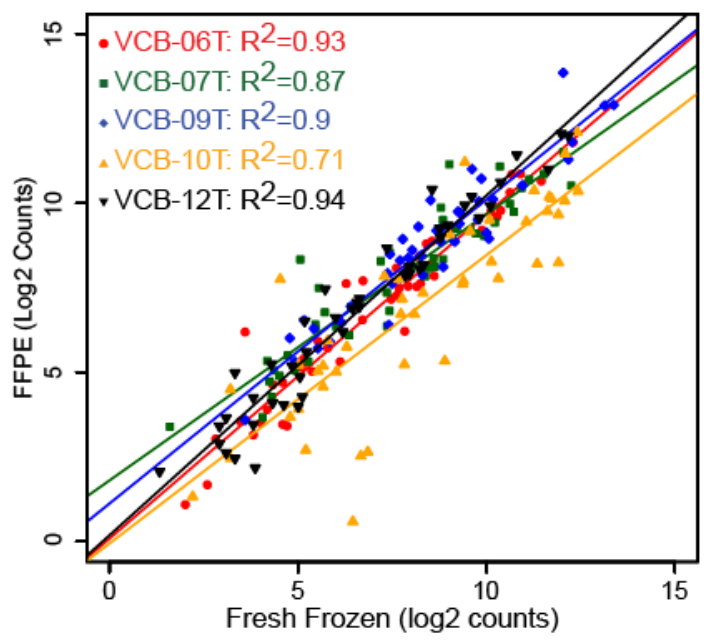

Figure 4.2 Assessing the fidelity of the Nanostring platform

(A) Linear regression analysis of gene expression values obtained from RNA-seq ( $x$-axis) with those obtained from Nanostring assay ( $y$-axis) performed on matching RNA isolated from fresh-frozen tissue. RNA-Seq values are presented as counts per million reads, all values are log transformed. (B) Linear regression analysis of gene expression values obtained from the Nanostring assay with RNA isolated from fresh-frozen tissue tissue ( $x$-axis) and FFPE tissue (y-axis) for matched samples. Coefficient of determination $\left(R^{2}\right)$ is shown for each comparison.

\subsection{Testing the Classifier}

To test the classifier performance, a cohort of 38 PPGL tumours was profiled on Nanostring (Table 4.3). Three tumours were from fresh frozen tissues and the remainder were archival FFPE cases. All tumours had either a) a known pathogenic germline mutation previously determined by Sanger dideoxynucleotide sequencing and formally reported by a genetic testing laboratory $(n=21), b)$ a likely pathogenic germline or somatic mutation within a known PPGL gene determined by massively parallel sequencing (targeted panel or exome) or Sanger dideoxynucleotide sequencing of tumour DNA ( $n=12)$, or c) functional SDH deficiency indicated by loss of SDHB expression by immunohistochemistry $(n=5)$. A MAX-driven tumour exhibiting regions of distinct histology was selected for sub-regional sampling to examine the effect of tumour heterogeneity on classification (Figure 4.3).

In summary, 34 of 38 tumours were classified to a subtype consistent with their known genotype or IHC staining phenotype (Table 4.3). Importantly, both macrodissected regions of the heterogeneous $M A X$ mutant case classified to the MAX-like subtype. SVM classification includes a probability score ranging from 0 to 1 for membership in each class, these values were used to establish confidence brackets (low, 
Table 4.3 Sample annotation and classification

\begin{tabular}{|c|c|c|c|c|c|c|c|}
\hline Sample & Source & P/PGL & Dx Age & Location & Site & Genotype & Six Class (Conf) \\
\hline RBH01-R1 & FFPE & PCC & 14 & Bilateral & RBH & MAX & MAX-Like (H) \\
\hline RBH01-R2 & FFPE & PCC & 14 & Bilateral & $\mathrm{RBH}$ & MAX & MAX-Like (M) \\
\hline $\mathrm{RBHO2}$ & FFPE & PCC & 50 & Right adrenal & RBH & MAX & MAX-Like $(H)$ \\
\hline RBH03 & FFPE & PCC & 42 & Bilateral & RBH & MAX & MAX-Like (H) \\
\hline RNSHO1 & FFPE & PCC & 35 & Right adrenal & RNSH & NF1 & RTK1 (H) \\
\hline RNSHO2 & FFPE & PCC & 45 & T3/T4 Spine & RNSH & SDHB & $\mathrm{SDHx}(\mathrm{M})$ \\
\hline RNSHO3 & FFPE & PCC & 41 & Omentum & RNSH & $S D H B$ & $\mathrm{SDH} x(\mathrm{H})$ \\
\hline RNSHO4 & FFPE & PGL & 40 & Retroperitoneal & RNSH & $S D H B$ & $\mathrm{SDHx}(\mathrm{H})$ \\
\hline RNSH05 & FFPE & PCC & 31 & Left adrenal & RNSH & HRAS & RTK3 (H) \\
\hline RNSHO6 & FFPE & PCC & 34 & Bilateral adrenal & RNSH & $V H L$ & VHL (H) \\
\hline RNSHO7 & FFPE & PGL & 35 & Retroperitoneal & RNSH & $S D H B$ & $\mathrm{SDHx}(\mathrm{M})$ \\
\hline RNSH08 & FFPE & PCC & 31 & Right adrenal & RNSH & $V H L$ & VHL (M) \\
\hline RNSH09 & FFPE & PCC & 39 & Right adrenal & RNSH & VHL & VHL (H) \\
\hline RNSH10 & FFPE & PCC & 40 & Left adrenal & RNSH & VHL & VHL (L) \\
\hline RNSH11 & FFPE & PCC & 24 & Adrenal & RNSH & TMEM127 & RTK1 (H) \\
\hline RNSH12 & FFPE & PCC & 37 & Bilateral adrenal & RNSH & TMEM127 & RTK3 (H) \\
\hline RNSH13 & FFPE & PCC & 31 & Right adrenal & RNSH & $R E T$ & RTK3 (H) \\
\hline RNSH14 & FFPE & PCC & 37 & Left adrenal & RNSH & RET & RTK1 (H) \\
\hline RNSH15 & FFPE & PGL & 27 & Left adrenal & RNSH & SDHB IHC Neg & $\mathrm{SDH} \times(\mathrm{L})$ \\
\hline RNSH16 & FFPE & PCC & 60 & Carotid & RNSH & SDHB IHC Neg & $\mathrm{SDHx}(\mathrm{H})$ \\
\hline RNSH17 & FFPE & PGL & 21 & Retroperitoneal & RNSH & SDHB IHC Neg & $\mathrm{SDH} \times(\mathrm{H})$ \\
\hline RNSH18 & FFPE & PGL & 35 & Carotid & RNSH & SDHB IHC Neg & $\mathrm{SDHx}(\mathrm{M})$ \\
\hline RNSH19 & FFPE & PCC & 41 & Left adrenal & RNSH & $S D H B$ & $\mathrm{SDHx}(\mathrm{H})$ \\
\hline VCB.01T & FFPE & PCC & 57 & Right adrenal & VCB & RET & RTK1 (M) \\
\hline VCB.04T & FFPE & PCC & 24 & Bilateral & VCB & $V H L$ & RTK2 (M) \\
\hline VCB.06T & FFPE & PCC & 17 & Right adrenal & VCB & $R E T$ & RTK1 (H) \\
\hline VCB.07T & FFPE & PCC & 60 & Right adrenal & VCB & HRAS & RTK1 (H) \\
\hline VCB.09T & FFPE & PCC & 76 & Left adrenal & VCB & RET & RTK2 (H) \\
\hline VCB.10T & FFPE & PCC & 31 & Right adrenal & VCB & NF1 & RTK1 (H) \\
\hline VCB.11T & FFPE & PCC & 56 & Right adrenal & VCB & NF1 & RTK3 (H) \\
\hline VCB.13T & FFPE & PCC & 26 & Right adrenal & VCB & $V H L$ & VHL (M) \\
\hline VCB.14T & FFPE & PCC & 34 & Bilaterlal & VCB & VHL & VHL (M) \\
\hline WM01 & Fresh & PCC & 7 & ND & $\mathrm{WCH}$ & $V H L$ & MAX-Like (L) \\
\hline WM02 & Fresh & PCC & 8 & ND & $\mathrm{WCH}$ & $V H L$ & MAX-Like (L) \\
\hline WM03 & FFPE & PCC & 3 & ND & $\mathrm{WCH}$ & $V H L$ & VHL (M) \\
\hline WM04 & FFPE & PCC & 13 & ND & $\mathrm{WCH}$ & $V H L$ & VHL (M) \\
\hline WM05 & FFPE & PCC & 8 & ND & $\mathrm{WCH}$ & VHL & RTK2 (M) \\
\hline WM06 & Fresh & PCC & 10 & ND & $\mathrm{WCH}$ & $V H L$ & $\mathrm{VHL}(\mathrm{H})$ \\
\hline WM07 & FFPE & PGL & 7 & ND & $\mathrm{WCH}$ & SDHB IHC Neg & $\mathrm{SDH} \times(\mathrm{M})$ \\
\hline
\end{tabular}

RNSH = Royal North Shore Hospital; RBH = Royal Brisbane Hospital; WCH = Westmead Children's Hospital; VCB = Victorian Cancer Biobank; ND = No Data; IHC Neg = Immunohistochemistry Negative; $(\mathrm{L} / \mathrm{M} / \mathrm{H})=\mathrm{Low} / \mathrm{Medium} / \mathrm{High}$ confidence. 


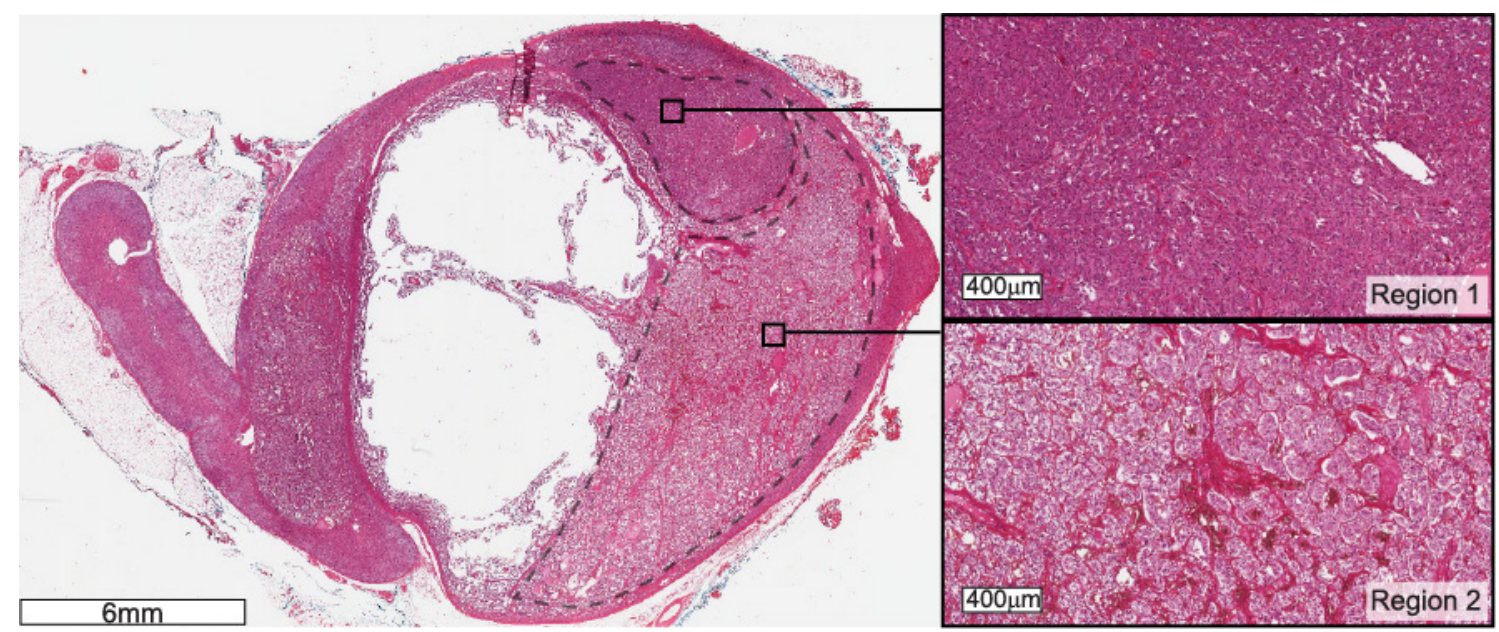

Figure 4.3 Histological heterogeneity in a MAX -driven tumour

Haematoxylin and eosin stained section of a MAX mutant tumour exhibiting distinct patterns of cellular organisation. Demarcated regions were macrodissected for RNA extraction. Region one exhibits plump spindled cells more typical of a MAX-driven tumour, region two exhibits a more zellballen-type histology.

0-0.5; medium, $>0.5-0.75$; high, $>0.75$ ). Of the four incorrect predictions all were $V H L$ PCCs and were predicted with either low or medium confidence to either RTK2 or MAX-like subtypes.

\subsection{Understanding the RTK Subtypes}

In order to understand why mutations in the MAPK/RTK signalling genes separate into three distinct subtypes (RTK 1, 2, and 3), the genes differentially expressed between these groups were examined. Pathway analysis of the genes most differentially expressed in the RTK2 subgroup using the MetaCore platform (GeneGo, Thomson Reuters, NY, USA) revealed that many of these genes belonged to the cortisol biosynthesis pathway. This metabolic pathway is highly active in the cells of the adrenal cortex. The most likely explanation for the high expression of these genes in the RTK2 subgroup is contamination with adrenocortical cells during sample preparation. To test this, a matched FFPE specimen block was obtained for an RTK2 case previously classified using RNA-seq data from a fresh frozen specimen without macrodissection. Sections were cut and stained with either hematoxylin and eosin or Chromogranin-A. Nanostring analysis of RNA from the macrodissected whole tumour region (Figure 4.4a) showed a moderate reduction in cortisol biosynthesis associated genes, while a smaller internal region (Figure 4.4b) displayed a precipitous reduction in expression (Figure 4.4c). A second case that had previously classified into the RTK2 subtype was found to be negative for Chromogranin-A. Upon pathological review the tumour was determined to have histological features more consistent with a zona reticularis type adrenal cortical adenoma (ACA), as such this case was excluded from further 


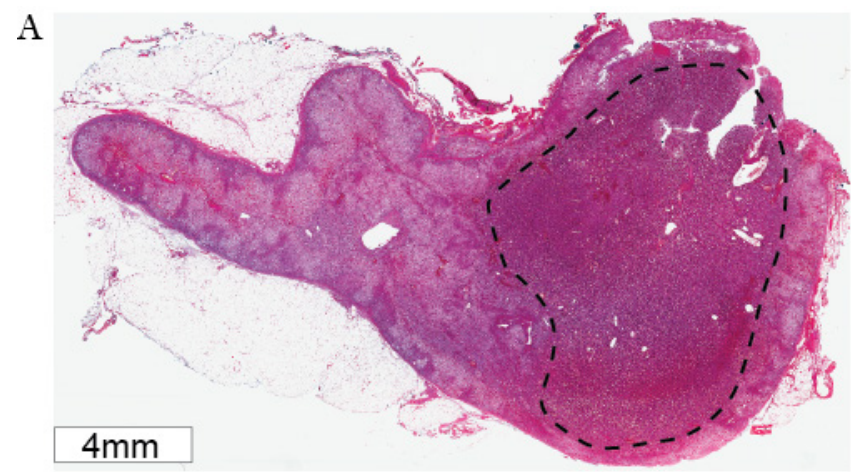

$\mathrm{C}$

$\mathrm{B}$
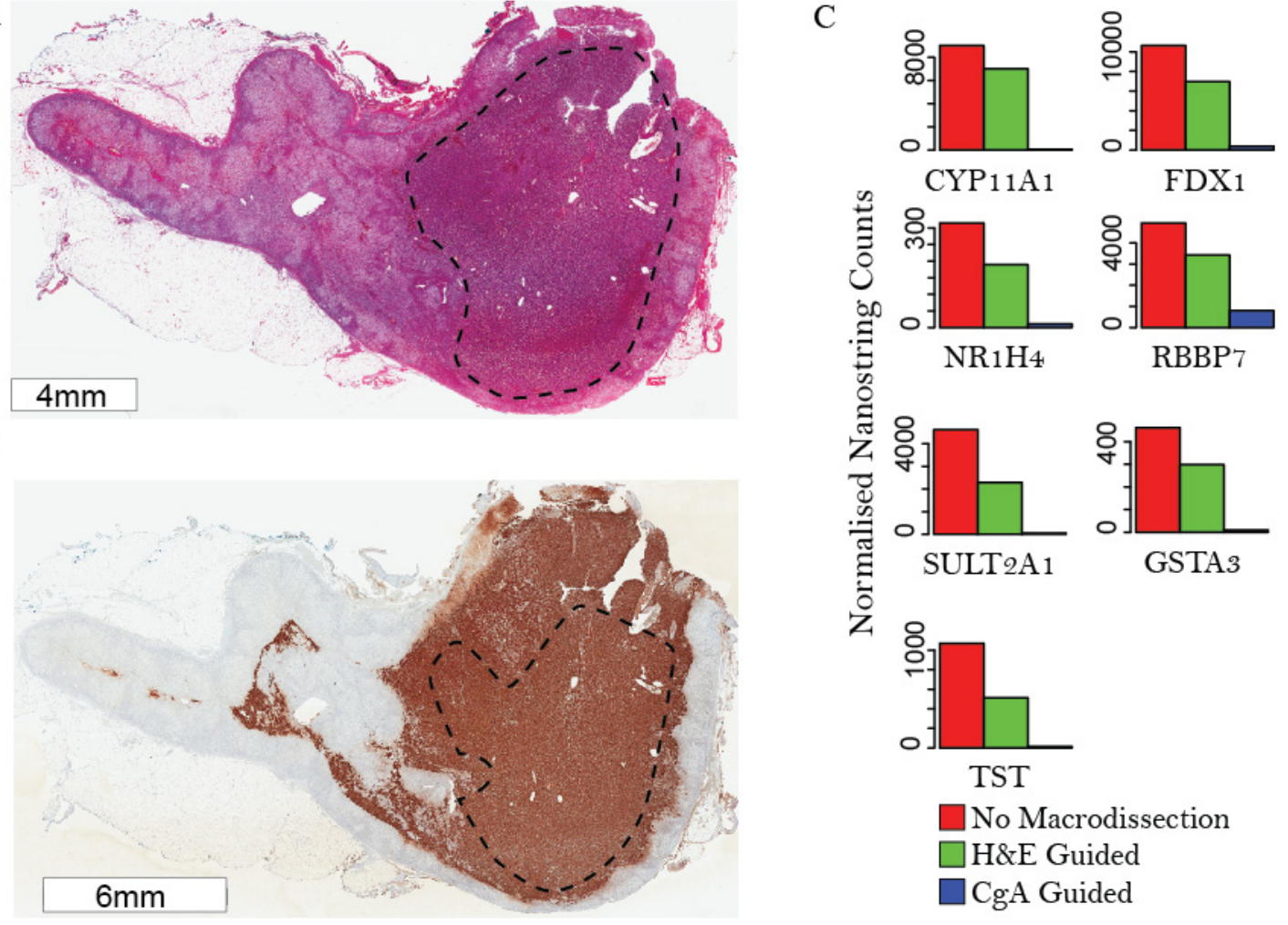

Figure 4.4 Elimination of RTK2 gene expression through chromogranin-A guided macrodissection

(A) Haematoxylin and Eosin (H\&E), and (B) Chromogranin-A antibody staining of a sample classified into the RTK2 subgroup, the regions used for macrodissection are demarcated with dashed lines. (C) Nanostring expression values for the genes representing the RTK2 subtype from fresh frozen tissue without macrodissection (red), H\&E guided macrodissection (green), and Chromogranin-A guided macrodissection (blue) of a matched FFPE specimen.

analysis. These results suggest that the RTK2 subtype detected in previously published microarray studies results from the presence of cortical tissue or, perhaps in rare cases, a misdiagnosis of a tumour of andrenocortical origin. The failure to abolish cortisol biosynthesis gene signature through dissection to the tumour margins may indicate a technical failure or a possible admixture of cell types at the periphery of the tumour.

Examination of the genes distinguishing the RTK3 subtype from RTK1 revealed a number of genes involved in immune function (CCR1, CD14, CSF1R, VAMP8, CTGF, TYROBP, SRGN). For many of these genes, the elevated expression relative to the RTK1 subgroup is also present in the VHL and, to a lesser degree, the SDHx subtypes. As tumours in the pseudo-hypoxia group are known to have extensive 
vascularisation resulting from activation of HIF signalling, the elevation of these immune related genes may be the result of increased immune infiltrate rather than a feature of the neoplastic cells themselves.

\subsection{Refining the Subtypes}

Given the potential contribution of non-neoplastic cells to the gene-expression profiling, it was reasoned that removal of the RTK2 subtype from analysis might improve classification stability for the purpose of genotype prediction. To this end, a five-class model was derived by removing the samples and genes representing the RTK2 class from the training set. Furthermore, as the aim was to provide clinically informative classification, additional four-class, consolidating the RTK1 and RTK3, and then three class, consolidating VHL and SDHx were tested (Figure 4.5a, Table 4.4, and Appendix B). Removal of RTK2 genes and tumours from the classifier training data improved the classification performance on test samples and increased the proportional number of medium- to high-confidence predictions (Figure 4.5b). Importantly, of three cases previously classified to RTK2, one RET case now classified to RTK3/RTK (high probability), one $V H L$ case to the VHL subtype (medium probability) and another VHL case to the SDHx subtype (low probability) using either five or four-class models. The three-class model consisting of RTK, MAX-like and a single pseudo-hypoxia subtype accurately classified all test samples with no low confidence predictions.

Table 4.4 Number of samples in each class by driver mutation

\begin{tabular}{|c|c|c|c|c|c|c|c|c|}
\hline & \multirow{3}{*}{ HRAS } & \multirow{3}{*}{ NF1 } & \multirow{3}{*}{ RET } & \multirow{3}{*}{ TMEM127 } & \multirow{3}{*}{$\begin{array}{c}\text { MAX } \\
4\end{array}$} & \multirow{3}{*}{ SDHB/IHC- } & \multirow{3}{*}{$\begin{array}{c}V H L \\
2\end{array}$} \\
\hline & & & & & & & & \\
\hline \multirow{6}{*}{ 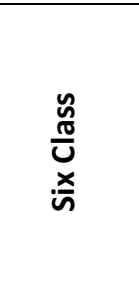 } & MAX-Like & & & & & & & \\
\hline & RTK1 & 1 & 2 & 3 & 1 & & & \\
\hline & RTK2 & & & 1 & & & & 2 \\
\hline & RTK3 & 1 & 1 & 1 & 1 & & & \\
\hline & SDHx & & & & & & 10 & \\
\hline & VHL & & & & & & & 9 \\
\hline \multirow{5}{*}{$\begin{array}{l}\text { पू } \\
\frac{\pi}{U} \\
\stackrel{0}{Z}\end{array}$} & MAX-Like & & & & & 4 & & 2 \\
\hline & RTK1 & 1 & 2 & 3 & 1 & & & \\
\hline & RTK3 & 1 & 1 & 2 & 1 & & & \\
\hline & SDHx & & & & & & 10 & 1 \\
\hline & VHL & & & & & & & 10 \\
\hline \multirow{4}{*}{ 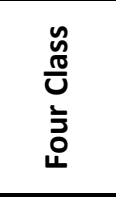 } & MAX-Like & & & & & 4 & & 2 \\
\hline & RTK & 2 & 3 & 5 & 2 & & & \\
\hline & SDHx & & & & & & 10 & 1 \\
\hline & VHL & & & & & & & 10 \\
\hline \multirow{3}{*}{ 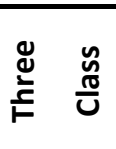 } & MAX-Like & & & & & 4 & & \\
\hline & pHyp & & & & & & 10 & 13 \\
\hline & RTK & 2 & 3 & 5 & 2 & & & \\
\hline
\end{tabular}

Note: See Figure 4.5 for structure of five, four, and three class classifiers 
A
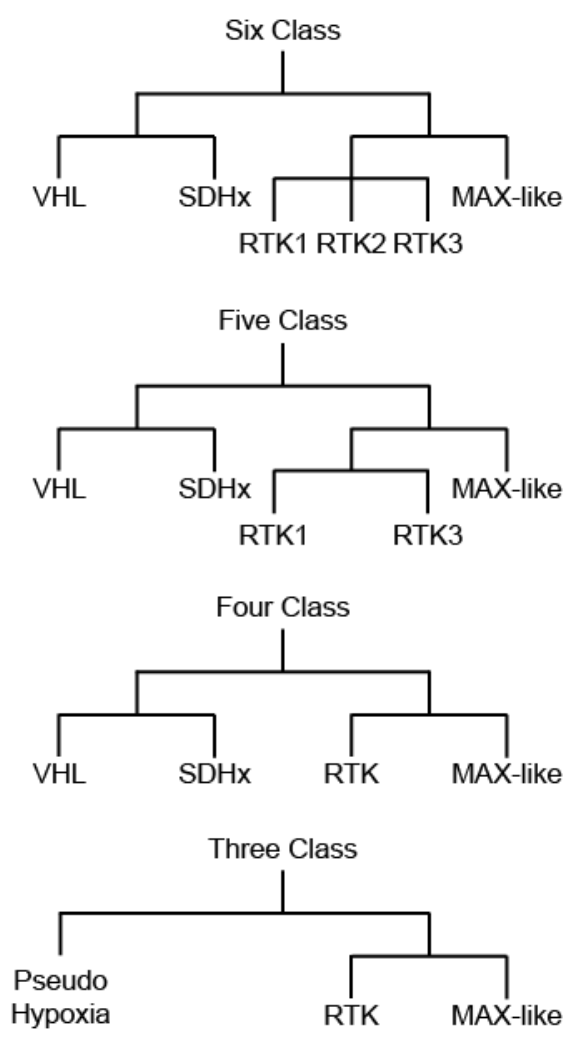

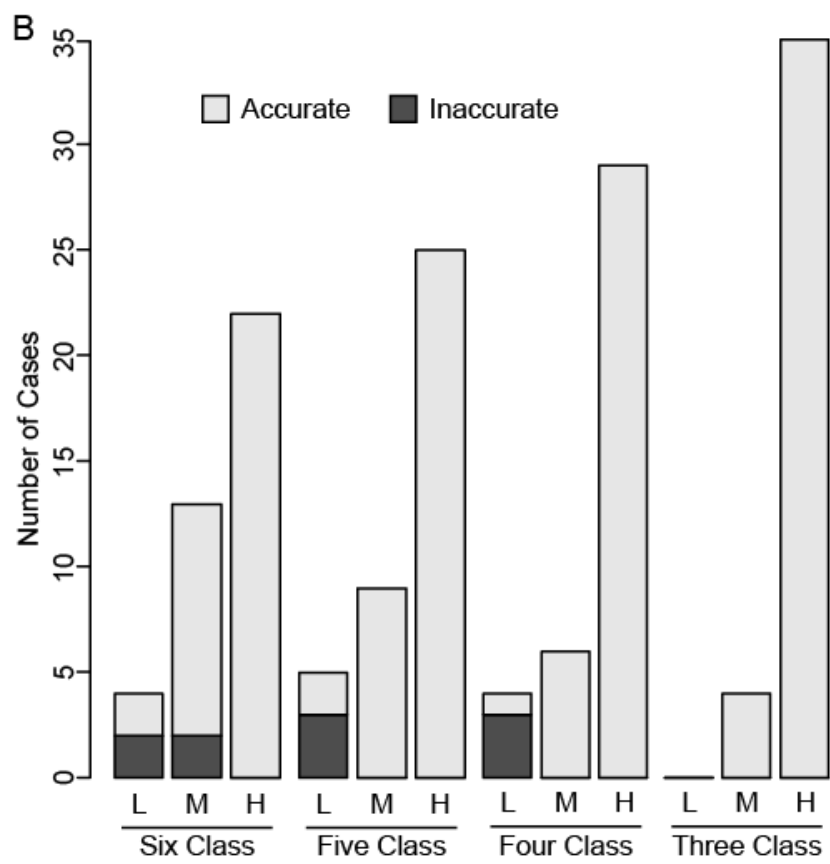

Figure 4.5 A comparison of classifier models for prediction of PPGL subtypes

A) Schematic representation of classifier models used B) Accuracy and distribution of prediction probability levels between classifier models ( $L=$ low probability, $M=$ medium probability, $H=H i g h$ probability).

\subsection{Discussion}

This chapter described the development and validation of a new clinical diagnostic test for classification of PPGL tumours based on the genetic driver of the disease. The assay has clear clinical utility in guiding genetic testing, providing supporting evidence in assessing pathogenicity of VUS, and providing orthogonal confirmation of molecular testing before major management decisions are made. In cases where a candidate causative mutation cannot be determined, the assay may still provide clinical guidance. For example, patients with disease that has been classified into a subtype with higher metastatic risk may be placed under greater surveillance. There is also the potential to guide selection of targeted therapies in metastatic disease based on subtype, for example, anti-angiogenic agents such as sunitinib are being considered for the treatment of SDH-type tumours (Jimenez et al., 2013). 
Through the process of refining the classifier this chapter has also provided further elucidation of previously established subtypes. The RTK2 subgroup, also known in the literature as Cluster $2 \mathrm{C}$, was shown to result from the presence of adrenocortical tissue. At this juncture it is unclear whether this is always a technical failure in isolating pure tumour tissue, or whether in some cases it may represent an admixture of cortical and chromaffin cells at the periphery of the tumour. Resolving this issue will likely require profiling of a larger set of tumours following careful Chromogranin-A guided macrodissection. As noted in Table 4.1, the cluster denoted Cluster 2A in previously published analysis, was further divided into the RTK1 and RTK3 subtypes in the analysis presented in Chapter Three. Although both subtypes are characterised by high expression of genes consistent with well-differentiated disease (e.g. $P N M T$ and RET), the RTK3 also exhibits elevated expression of genes associated with inflammation and wound repair (e.g. CSF1R, TYROBP, CCL2, CYR61, CTGF). While recombining the RTK 1 and 3 subtypes in a four-class model is efficacious for the purposes of genotype detection, it is best to be cautious in dismissing this subgroup as interactions between the immune system and cancer are highly complex. The immune system provides the first line of defence in detecting and eliminating nascent tumours; development of mechanisms to evade the immune system through immunoediting are a key part of tumour development, as such the immune system is now considered a major selective pressure in tumour evolution (Mittal et al., 2014). Conversely, in some scenarios inflammation has been shown to promote tumour growth and development, a process that may involve activation of pro-growth and survival signals or the creation of a permissive microenvironment (Chow et al., 2012; Rakoff-Nahoum, 2006). Until more is known regarding the role of immune infiltrates in PPGL, it seems judicious to retain the ability to detect samples in the RTK3 class.

MAX-like/Cluster-2B tumours have a distinct gene-expression signature and the membership of $M A X$ tumours in this subtype is further evidenced by the data presented in this chapter. However, it is evident that MAX mutant PPGL tumours can be phenotypically heterogeneous as shown by the single case presented in this chapter. Despite successful subtyping of both regions using the Nanostring classifier, there were notable differences in gene-expression between these tumour regions with Region Two exhibiting raised expression of the immune signature genes (CCL2 and CYR61) which distinguish RTK3 from RTK1. Notably, a recent publication by Castro-Vega et al. (Castro-Vega, Letouze, et al., 2015) included the classification of three $M A X$-mutant tumours that were classified to $\mathrm{C} 2 \mathrm{~A}, \mathrm{C} 2 \mathrm{~B}$ and $\mathrm{C} 2 \mathrm{C}$ subtypes respectively, although the latter is likely due to presence of adrenocortical tissue in the sample masking a true subtype association for this case as previously discussed. Previous analysis of an independent microarray data set described by Lopez-Jimenez et al. (see Chapter Three Figure 4c) also suggested that a proportion of $M A X$-mutant cases were classified outside of the MAX-like/C2B group. A 
larger number of $M A X$-mutant PPGL tumours will need to be gene-expression profiled to determine the distribution of $M A X$-mutant PPGL across Cluster 2 subtypes.

The current implementation of the classifier relies on the use of existing microarray data as a training set. While this approach has given encouraging results, it still relies on data transformation techniques that can skew the data if the training or testing set is undersized or a particular class is underrepresented. Ultimately, the most robust approach will involve gathering a sufficiently well annotated Nanostring data set that can be used as a training set for testing new samples. This may also facilitate detection of genotypes that are underrepresented in the current training data set, such as $F H$ and EPAS1, providing a sufficient number of samples can be profiled.

In summary, this chapter has demonstrated that accurate subtyping and genotype prediction is possible from FFPE tissue specimens. Moving forward, a prospective multisite trial, assessing factors such as sample fixation methods, equipment and reagent variation, and operator bias, will be required to gauge the utility, accuracy, and robustness of the assay for clinical purposes. 


\section{Inter- and intra-tumoural heterogeneity in the context of syndromic neuroendocrine tumours}

\subsection{Chapter Preface}

In the mid-nineteenth century, Charles Darwin proposed the theory of natural selection. The theory postulated that individuals of a species, if possessive of a novel trait advantageous for survival would thrive and be more likely to produce offspring, thus preserving the trait in future generations. At the time Darwin was unable to provide an underlying mechanism for heredity, it was not for another century that experiments using Pneumococcus (Avery et al., 1944) would demonstrate that hereditary traits were transmitted by genetic information encoded in DNA. Since then, DNA sequencing and other technological advances have led to an understanding of how the accumulation of small alterations to the structure of DNA provides the mechanism for diversification, adaption and speciation. However, despite the critical role of mutations in the continued evolution of life, beneficial alterations to genetic material are the minority, with most being either neutral or deleterious. In the early 20th century, even without knowledge of the intricacies of DNA, German biologist Theodor Bovari recognised a role for deleterious alteration to genetic material, specifically errors in its replication, in cancer development after observing abnormal mitosis in sea urchin eggs (Boveri, 2008). In the subsequent decades, cytological experiments continued to refine the understanding of how abnormal chromosomal structure contributed to oncogenesis with studies making note of the dynamic nature of the cancer genome (Sato, 1950). For a period of time, it was debated as to whether tumours arose from a single cell (monoclonal) or from a group of cells (polyclonal). In 1967, David Linder and Stanley Gartler presented an elegant experiment demonstrating that the same copy of the $\mathrm{X}$ chromosome was inactivated in all cells of a uterine leiomyoma, thus providing strong evidence in support of the monoclonal origin of tumours. A decade later, based on observations that tumours, although originating from a single cell, display diverse chromosomal patterns, Peter Nowell hypothesised that cancer cells may undergo processes of natural selection and evolution similar to those proposed for speciation (Nowell, 1976). Nowell proposed that the accrual of genetic alterations ranging from point mutations to major chromosomal aberrations was correlated with the progression of cancer through the stages of malignancy. However, at the time, Nowell lamented that the current mapping of the human genome was too incomplete to make any correlation at the level of the chromosome.

Since then, the intervening decades have brought technological advances ranging from Sanger dideoxynucleotide sequencing to high-resolution microarray based cytogenetic assays. However, it is only 
in the last decade that massively parallel sequencing technologies have facilitated the mapping of entire genomes at base pair resolution at an affordable cost. The capabilities of this technology have fuelled a renewed effort towards understanding the phenomena of tumour evolution. Recent years have seen the publication of studies examining clonal evolution in tumours of the colon (Kim, Jung, et al., 2015), prostate (Boutros et al., 2015; Kim et al., 2014), lung (Jamal-Hanjani et al., 2014; Zhang et al., 2014), kidney (Fisher et al., 2014), ovary (Bashashati et al., 2013), and brain (Kim, Zheng, et al., 2015). These explorations of tumour heterogeneity provide insight into the selective pressures acting on a developing tumour, as well as demonstrating examples of evolutionary convergence that highlight potentially obligate oncogenic events. For example, spatial sequencing of a renal cell carcinoma (RCC) revealed that several distinct mutations in both the histone methyltransferase SETD2 and the histone demethylase $K D M 5 C$ had arisen independently in spatially separate regions of the tumour (Gerlinger et al., 2012). Findings such as these suggest that tumours may be reliant on the disruption of specific genes or pathways, thus creating oncogenic dependencies that may present common therapeutic vulnerabilities between tumour clones. The concept of cancer genomes as dynamic and evolving has enormous implications for cancer therapy, especially with regard to treatment resistance and metastatic potential. Current thinking suggests a small number of resistant clones may already be present within a tumour at the beginning of a treatment cycle (Cooke et al., 2011; McGranahan et al., 2015), wherein the application of a therapeutic agent acts as a selective pressure eliminating cells lacking the adaption, enabling outgrowth of the resistant cells (Bozic et al., 2013). This paradigm has fostered the idea of using orthogonal combination therapies targeting diverse pathways simultaneously, limiting the efficacy of treatment escape mechanisms (Holohan et al., 2013).

Tumour heterogeneity, a concept that encompasses both intertumoural and intratumoural heterogeneity, is one of the greatest challenges facing modern cancer therapies. Increasingly, higher degrees of intratumoural heterogeneity are being linked to less favourable outcome (Mroz \& Rocco, 2013; Zhang et al., 2014), presumably by providing a more diverse repertoire with which to respond to environmental challenges, whether that be intrinsic changes in the tumour microenvironment or assault from external sources such as targeted or chemotherapeutic agents. Meanwhile, intertumoural heterogeneity can lead to disparate outcomes in patients receiving identical treatment for ostensibly the same disease, obfuscating clinical best practice.

Chapter Three examined the breadth of genomic variation present in independent tumours arising across a diverse patient population. While comparison between patients can provide valuable insight into obligate oncogenic events, the inherent genetic variation between patients may obscure the patterns of tumour 
evolution that give rise to heterogeneity. For this reason, synchronous primaries arising from familial tumour syndromes such as MEN2A, von Hippel-Lindau, and the Paraganglioma Syndromes provide an exceptional opportunity to explore the diversity of tumour evolution given a uniform antecedent. Fisher and co-workers likened this scenario to a thought experiment proposed by evolutionary biologist Stephen Jay Gould (Fisher et al., 2014). Gould suggested that steps within an evolutionary trajectory were contingent on prior events and that if one were able to reset the system, restarting with identical conditions, one could expect a different evolutionary course to unfold. In contrast, the concept of evolutionary convergence suggests that identical selective pressures should ultimately result in a similar evolutionary trajectory. Perhaps unexpectedly, in their exploration of synchronous RCC arising from a VHL mutation, Fisher et al. observed that rather than operating in a mutually exclusive manner, instances of contingent and convergent evolution were present and complementary. In PPGL, copy-number profiling has been used by a small number of studies to examine tumour evolution through the lens of intratumoural heterogeneity, examining both the relationship between regions within a primary and between a primary and related metastases (Castro-Vega, Lepoutre-Lussey, et al., 2015; Crona et al., 2015). However, how evolutionary concepts such as contingency and convergence contribute to the development of synchronous PPGL has yet to be explored.

It is well know that familial tumour syndromes exhibit tissue specificity but less is known about why inherited gene mutations only affect cells of a particular origin (Maris \& Knudson, 2015). In the context of PPGL it has been shown that the underlying driver mutation is predictive of secondary genomic events likely to occur during tumourigenesis (Castro-Vega, Letouze, et al., 2015; Crona et al., 2015). As such, it stands to reason that under the control of a given driver gene, oncogenic transformation may exhibit convergent events regardless of cell type. An understanding of these events may shed light on core genetic alterations required for progression regardless of cell of origin, thus providing clues to tissueagnostic therapeutic targets. Synchronous primaries originating from diverse tissue types provide an invaluable opportunity to examine the relationship between driver mutation, cell of origin, and tumour genome evolution.

Using high-resolution copy-number profiling and exome sequencing to map tumour heterogeneity at base pair resolution, this chapter will present two case studies examining the genomic landscape of multifocal disease in the context of a germline driver mutation. 


\subsection{Case One: Paragangliomas-4 (PGL4)}

\subsubsection{Patient Presentation}

A 23-year-old male with a history of flushing, pounding headache, paroxysms of palpitations, nausea, and vomiting presented with sudden back pain and elevated blood pressure. Renal ultrasound revealed a paraaortic mass triggering a subsequent CT scan which demonstrated four retroperitoneal masses; a soft tissue mass located immediately above the left renal vein contiguous with the left adrenal gland and anterior to the left renal hilum ( 43 x $46 \mathrm{~mm}$, designated primary one), a left para-aortic mass at L2 (17 x $22 \mathrm{~mm}$, designated primary two), and aortocaval masses at the levels of L2 (32 x $36 \mathrm{~mm}$, designated primary three) and L3/4 (29 x $34 \mathrm{~mm}$, designated primary four). Twenty-four hour urinary catecholamine levels showed elevated norepinephrine $(10.2 \mu \mathrm{mol} / 24 \mathrm{hr}$; normal range: 0-0.8 $\mu \mathrm{mol} / 24 \mathrm{hr})$. PET/CT imaging with

${ }^{18}$ F-FDG demonstrated intense avidity in all lesions (Figure 5.1a), extensive diffuse mesenteric and retroperitoneal activity was seen elsewhere in the abdomen with no CT correlate, likely representing physiological brown fat activation by excess catecholamine secretion. Additional staging with GaTate $\left({ }^{68} \mathrm{Ga}\right.$-DOTA-octreotate) demonstrated strong avidity in primaries three and four, while primary one demonstrated heterogeneous uptake suggestive of necrotic regions, and primary two demonstrated minimal avidity (Figure 5.1b). An additional $7 \mathrm{~mm}$ soft tissue nodule anterior to the piriformis muscle was detected within a focus of strong FDG uptake. Following alpha and beta blockade with Phenoxybenamine $(80 \mathrm{mg} /$ daily) and Atenolol (50 mg/daily), the patient underwent laparotomy and en bloc resection of primaries one through four and removal of the left kidney and adrenal gland (Figure 5.1c). Surgical removal of the pelvic lesion anterior to the piriformis muscle was not attempted. Pathological review revealed the tumours to have an organoid appearance consisting largely of nests of partially discohesive epithelioid cells. Immunohistochemistry demonstrated positive staining for synaptophysin and Chromogranin A, and negative staining for cytokeratin (AE1/AE3) and SDHB. Genetic testing revealed a single-base frameshift deletion resulting in a premature stop codon in the SDHB gene (NP_002991: p.Gln30ArgfsTer47).

\subsubsection{Genomic Profiling}

Following surgical extraction, sections of each tumour were excised for genomic analysis (Figure 5.1d). Two longitudinal sections were excised from the largest primary approximately $1 \mathrm{~cm}$ either side of the central axis. A single longitudinal section was excised along the central axis of the remaining three tumours. The excised slivers were then further divided into two, three, or four segments depending on the 


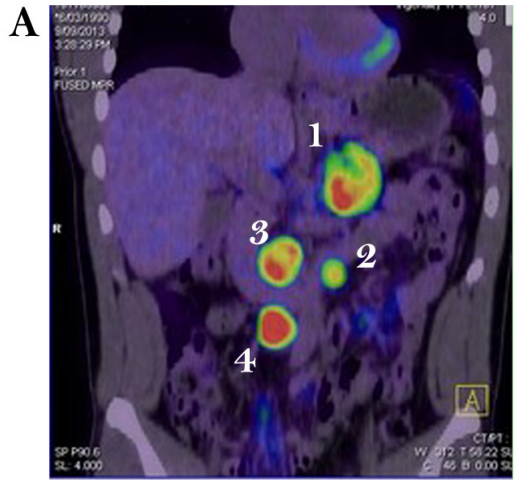

FDG

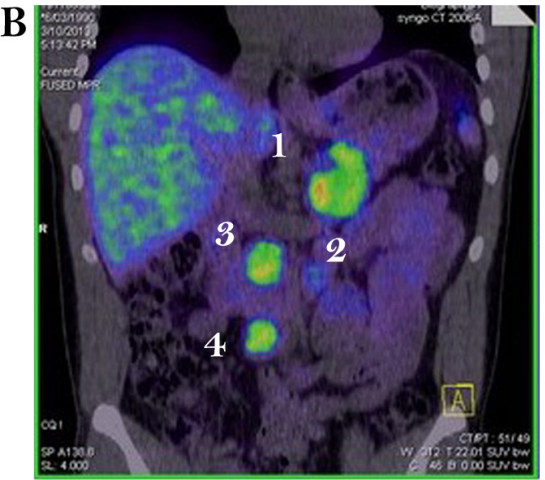

GaTate

C

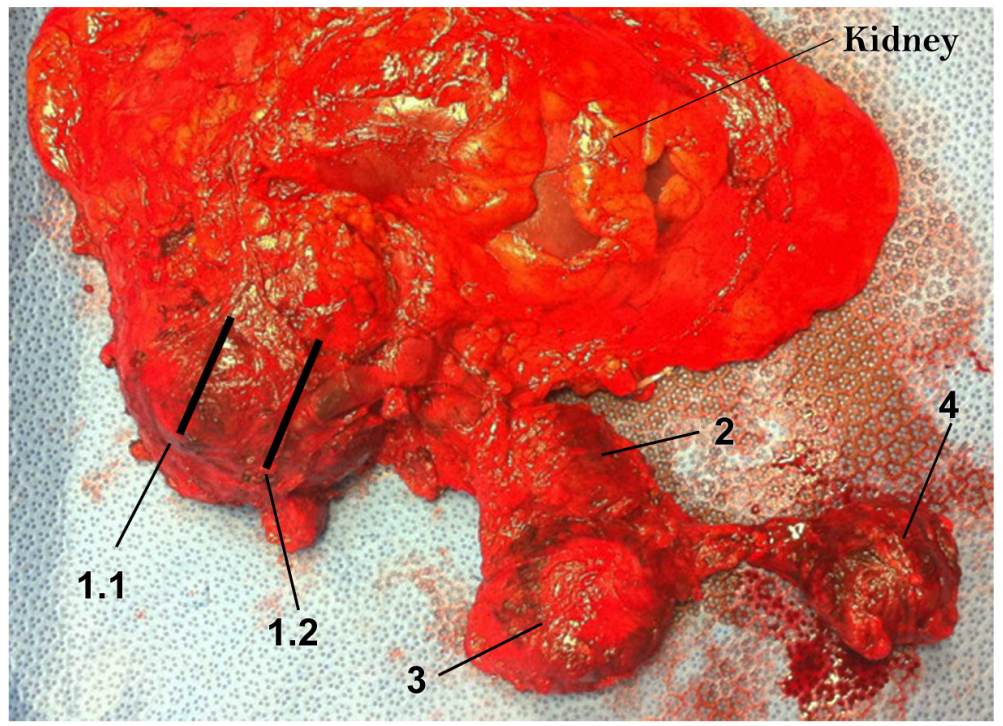

D

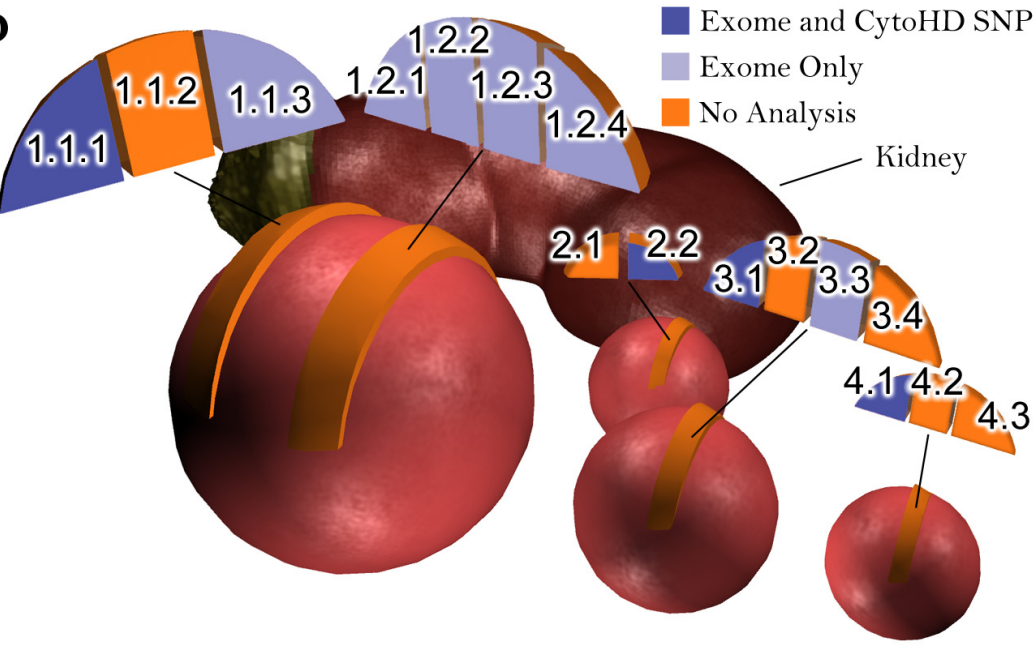

Figure 5.1 Anatomical view of synchronous PGL primary tumours

(A) 18F-FDG and (B) GaTate PET/CT showing in situ tumour location. (C) Kidney, adrenal gland, and multiple primaries following surgical resection. (D) Schematic of sample acquisition for multiregion genomic analysis. 
length of the sliver. A single piece from each tumour was selected for high-resolution copy-number profiling using CytoScan HD SNP array technology. Exome sequencing using the Agilent SureSelect V5 target enrichment platform was performed on six regions from primary one, two from primary three, and one from primaries two and four. Copy-number profiles were derived from exome sequencing data using the ADTEx algorithm (see Methods 2.3.2.2). High confidence somatic variants were determined using multiple mutation callers combined with an empirical filtering strategy (see Methods 2.3.1).

\subsubsection{Genomic Alterations}

Copy-number profiling revealed the presence of a small number of large-scale chromosomal loss events in each of the four primaries (Figure 5.2). Consistent with Knudson's two-hit hypothesis for tumour suppressor genes (Knudson, 1971), all four tumours exhibited single copy loss of the short arm of chromosome 1, indicating loss of the wild-type $S D H B$ allele. Loss of the long arm of chromosome 2 was apparent in primaries one and four. Alterations to chromosome 2 are relatively uncommon in PPGL, present in around 15\% of cases overall ((Crona et al., 2015), supplementary data) and around 5\% of those harbouring an $S D H B$ mutation ((Castro-Vega, Letouze, et al., 2015), supplementary data). Examination of the enrichment of heterozygous SNPs on chromosome 2 revealed that the same copy was lost in both tumours (Figure 5.3). Although statistically there is a $25 \%$ chance of a particular set of alleles being enriched in both tumours, this observation combined with the rarity of chromosome 2 alterations suggests that the loss may be enriching a deleterious mutation present in the germline. Analysis of the enriched deleterious (i.e. resulting in an amino acid change) germline mutations present on chromosome 2 revealed variants in 87 genes, mutations predicted to be benign using the SIFT, PolyPhen2, and Condel algorithms were removed, yielding a short list of 20 genes (Table 5.1). A number of the genes are representative of the ontologies presented in Chapter Three including neural development (HS6STI) and apoptosis (DAPL1, PRKRA), as well as genes involved in mitochondrial function (MFF, MTERFD2). However, given the currently limited understanding of oncogenic pathways in PPGL, it is difficult to implicate or eliminate genes with any certainty.

Degrees of subclonal loss of chromosome 3 were seen in primaries one, two, and three. Alterations to chromosome 3 are commonly found in PPGL, being present in up to 75\% of cases (Crona et al., 2015), supplementary data). The nature of the alteration is often correlated with the driver context, loss of the short arm, where $V H L$ is located, is present in over $85 \%$ of Cluster $1 \mathrm{~B}(V H L)$ tumours but only $13.4 \%$ of Cluster 2A (RET, NF1, HRAS) tumours, while loss of the long arm is present in around $65 \%$ of tumours 
A

Primary One Region One

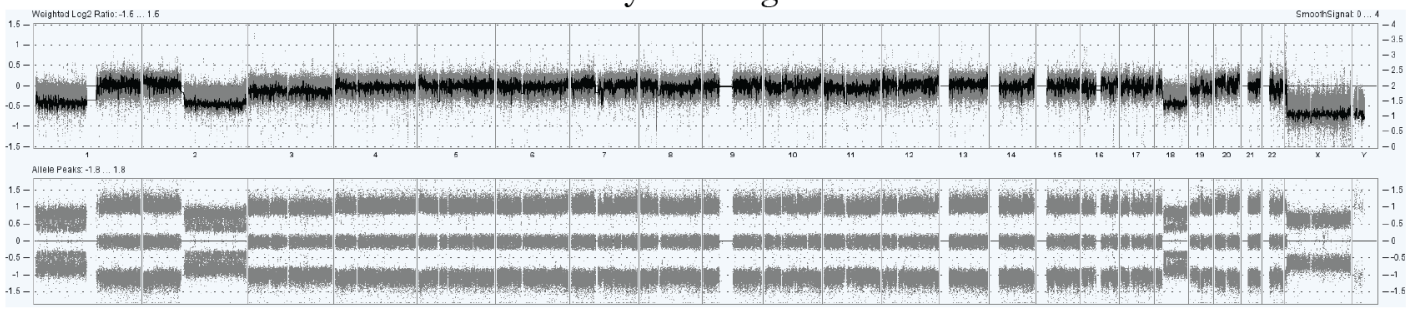

B

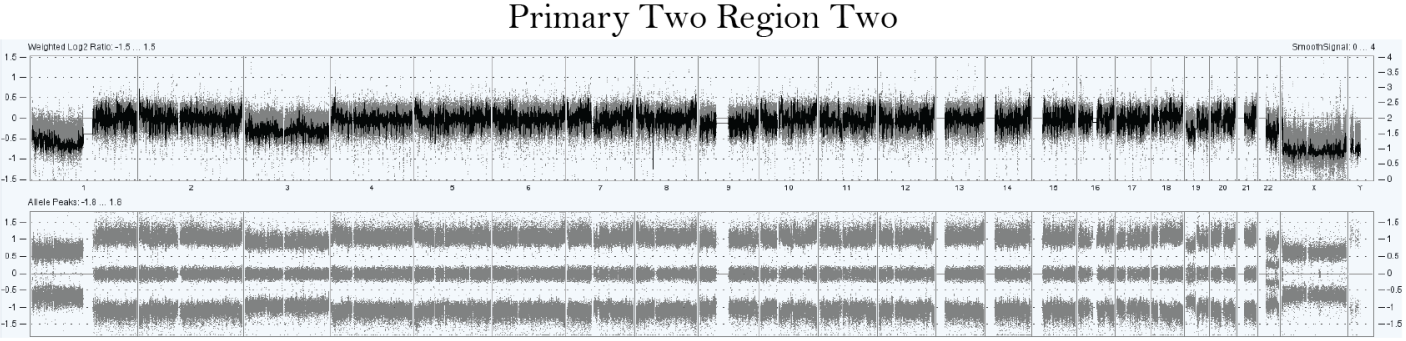

$\mathrm{C}$

Primary Three Region One

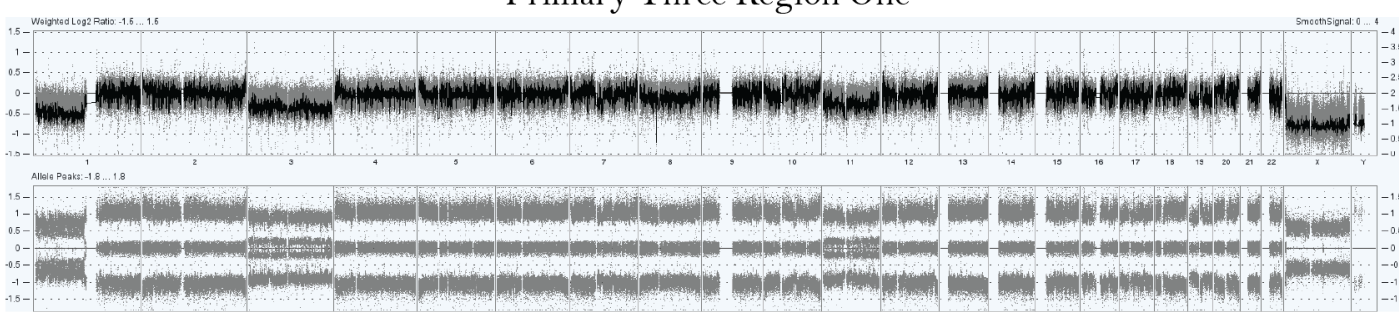

$\mathrm{D}$

Primary Four Region One

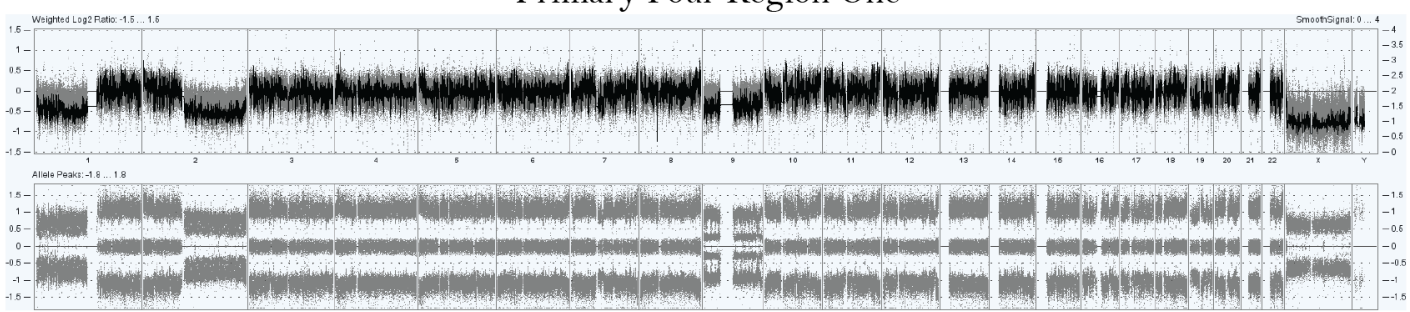

Figure 5.2 High resolution copy-number analysis of synchronous PGL

Copy-number profiling performed with the Affymetrix CytoscanHD platform on one region from each of the four synchronous primaries (a-d) in case study one. See Figure 5.1d for regions sampled.

in both clusters. Loss of either the short or long arms of chromosome 3 is less common in Cluster 1A $(S D H x)$ tumours, present in 18.8 and $25 \%$ of cases respectively. Interestingly, multiregional sampling of primaries one and three did not demonstrate enrichment of the loss event in any region sampled. Regions one ( $\operatorname{Pr} 3.1)$ and three ( $\operatorname{Pr} 3.3)$ of primary three showed a stable proportion of cells exhibiting the loss 

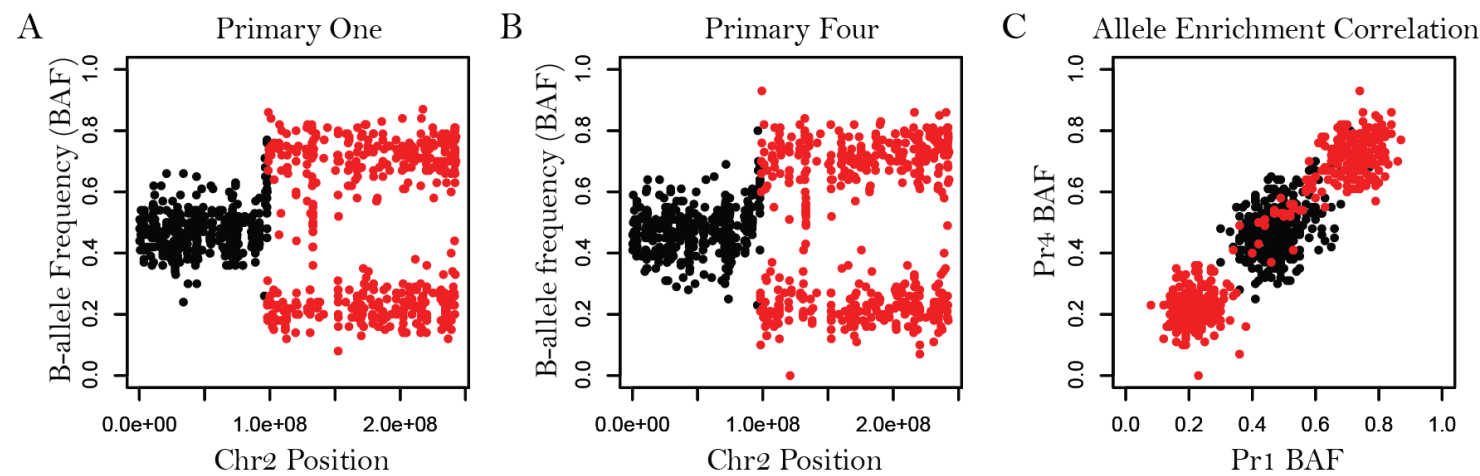

Figure 5.3 Allele enrichment resulting from chromosome $2 q$ loss

Germline SNPs were filtered to retain those with a read-depth greater than 50 in the tumour and germline, a B-allele frequency between 0.45 and 0.55 in germline, and a dbSNP identifier. Panels A and B show the BAF for SNPs on Chr2q in primaries one and four, respectively. Panel C shows the B-allele frequency for each corresponding SNP in primary one ( $x$ axis) and four ( $y$ axis). Data points are colour coded to indicate SNPs in regions of loss (red) and neutral copy-number (black). Positive correlation indicates identical allele sets (i.e. chromosomal copies) lost.

A

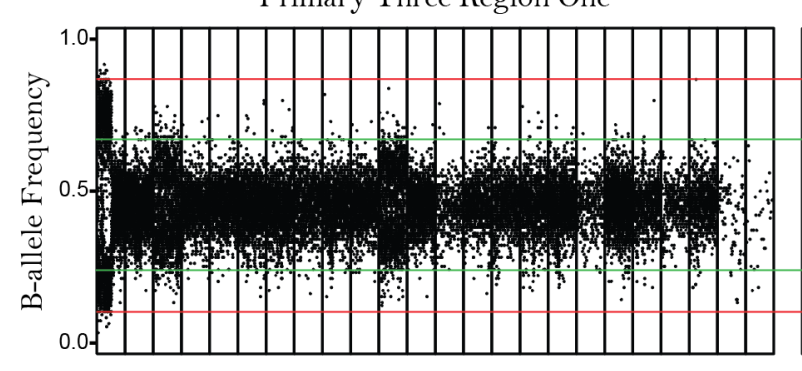

$1223455678910111213141516171819202122 \times Y$
B

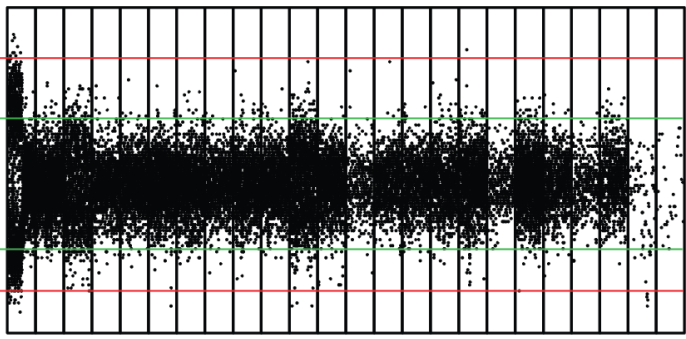

$1234456788910111213141516171819202122 \times Y$

Figure 5.4 Maintenance of clonal diversity

$B$-allele frequency plots for regions one $(A)$ and three $(B)$ of primary three derived from exome sequencing data using the ADTEx algorithm. In addition to clonal loss of chromosome $1 p$, subclonal loss events can be seen on chromosomes 3 and 11. Lateral lines indicate the approximate extent of the Ballele frequency shift caused by clonal (red) and subclonal (green) events.

event (Figure 5.4). Unfortunately, although detectable with high-resolution SNP array technology, the clonal proportion of the loss event in primary one was below the signal to noise ratio viable for ADTEx analysis. As a result, while it is possible to say the proportion of cells with chromosome 3 loss did not greatly increase in any region, it is not possible to determine if the proportion was maintained as was seen in primary three.

Loss of chromosome 11 was also present in primary three at a similar subclonal frequency to that of the chromosome 3 loss. It is not possible to determine from the genomic data whether both events occurred 
Table 5.1 Genes harbouring deleterious mutations enriched by loss of chromosome $2 q$

\begin{tabular}{|c|c|c|c|}
\hline $\begin{array}{l}\text { HGNC } \\
\text { Symbol }\end{array}$ & $\begin{array}{l}\text { Amino Acid } \\
\text { Change }\end{array}$ & Gene Description & Biological Process \\
\hline ANKRD36 & p.G465R & Ankyrin repeat domain 36 & \\
\hline RGPD4 & p.K823M & RANBP2-like and GRIP domain containing 4 & \\
\hline SULT1C3 & p.G179R & Sulfotransferase family, cytosolic, 1C, member 3 & $\begin{array}{l}\text { Sulfation } \\
\text { biotransformation } \\
\text { reaction }\end{array}$ \\
\hline$D B I$ & p.Y47* & $\begin{array}{l}\text { Diazepam binding inhibitor (GABA receptor modulator, } \\
\text { acyl-coa binding protein) }\end{array}$ & GABA signalling \\
\hline HS6ST1 & p.D87E & Heparan sulfate 6-O-sulfotransferase 1 & Neural development \\
\hline POTEJ & p.G683S & POTE ankyrin domain family, member J & \\
\hline DAPL1 & p.L60P & Death associated protein-like 1 & Apoptosis \\
\hline PRKRA & p.A8Rfs*22 & $\begin{array}{l}\text { Protein kinase, interferon-inducible double stranded RNA } \\
\text { dependent activator }\end{array}$ & Apoptosis \\
\hline FSIP2 & p.I514T & Fibrous sheath interacting protein 2 & \\
\hline$L R P 2$ & p.G669D & Low density lipoprotein receptor-related protein 2 & Endocytosis \\
\hline MFSD9 & p.1288T & Major facilitator superfamily domain containing 9 & \\
\hline MFF & p.S7C & Mitochondrial fission factor & $\begin{array}{l}\text { Mitochondrial } \\
\text { function }\end{array}$ \\
\hline C2orf83 & p.W141* & Chromosome 2 open reading frame 83 & \\
\hline SPATA3 & p.Q30_Q38del & Spermatogenesis associated 3 & \\
\hline $\mathrm{MROH} 2 \mathrm{~A}$ & p.E329G & Maestro heat-like repeat family member $2 \mathrm{~A}$ & \\
\hline OR6B2 & p.R122C & Olfactory receptor, family 6 , subfamily $B$, member 2 & Olfactory signalling \\
\hline MTERFD2 & p.T45A & MTERF domain containing 2 & $\begin{array}{l}\text { Mitochondrial } \\
\text { function }\end{array}$ \\
\hline$A G X T$ & p.P11L & Alanine-glyoxylate aminotransferase & $\begin{array}{l}\text { Glyoxylate } \\
\text { detoxification }\end{array}$ \\
\hline PASK & p.E796K & PAS domain containing serine/threonine kinase & $\begin{array}{l}\text { Energy homeostasis } \\
\text { and protein } \\
\text { translation }\end{array}$ \\
\hline
\end{tabular}

in the same subclone, so while it is likely that this is the case, it is also possible that the alterations have occurred reciprocally in a proportionate number of cells. Clonal loss of the long arm of chromosome 18 was present in primary one, while subclonal loss of chromosome 22 and the short arm of chromosome 19 were evident in primary two. Each of the primaries has a generally stable genome as is typical of PPGL, with primaries one to four having $17.3 \%, 12.9 \%, 14.8 \%$, and $13.4 \%$ of the genome altered, respectively. 
Similarly to the samples surveyed in Chapter Three, the copy-number changes are dominated by copy loss occurring as large segmental events, making it difficult to isolate the critical gene loci.

A low mutation burden and limited incidence of recurrent mutations was observed in each of the primaries, reflective of what has been seen both in the genomic survey conducted in Chapter Three and in subsequent publications (Castro-Vega, Letouze, et al., 2015). Primary one had the greatest number of single nucleotide coding region alterations with nineteen unique mutations, an average of 6 per region across the six regions sampled (range 2 to 9). Primary three had five unique coding mutations with an average of 4.5 mutations per region sampled (range 4 to 5). Primaries two and four, for which only a single region was sampled, had one and three coding mutations respectively. There were no commonly mutated genes between the tumours, however, several of the deleteriously mutated genes have been previously reported by others (Castro-Vega, Letouze, et al., 2015). Of particular interest is the secreted metalloprotease ADAMTS9, the epigenetic inactivation of which has been demonstrated in several cancer types (Peng et al., 2013; Zhang et al., 2010), correlating with poor prognosis in gastric cancer (Du et al., 2013). A second recurrently mutated gene, CSMD3, has been implicated in adult familial myoclonic epilepsy type 1 and autism suggesting a role in neural development or function (Floris et al., 2008; Shimizu et al., 2003), however, the gene is extremely large and, as such, has a greater opportunity to undergo spontaneous mutation, therefore, it may be over represented in mutation detection.

One current theory of tumour evolution is that of competition and selection, whereby a single clone accrues a genomic change, which provides a growth advantage allowing the clone to outgrow competing clones and become dominant. The implication of this is that the most advantageous genomic changes should be present in the largest number of cells, however, it does not necessarily follow that mutations found in the majority of cells are advantageous. In order to assess the relationship between ubiquity and mutation consequence, mutations were categorised as either deleterious or synonymous, and as ubiquitous (present in all regions sampled), shared (present in two or more but not all regions sampled), or private (present in only a single region) (Figure 5.5). Primary one had a single ubiquitous deleterious mutation, five deleterious and five synonymous shared mutations, and one synonymous and seven deleterious private mutations. Primary three had two deleterious and two synonymous ubiquitous mutations, and a single private deleterious mutation. Contrary to expectation, the ubiquitous and shared mutations do not demonstrate an enrichment of deleterious events comparative to private mutations; in fact, in the case of primary one the private mutations demonstrate a higher proportion of deleterious events. The equal distribution of synonymous and deleterious mutations amongst the shared mutations may be reflective of mutational timing, whereby a clone obtains a synonymous mutation prior to a deleterious one and 


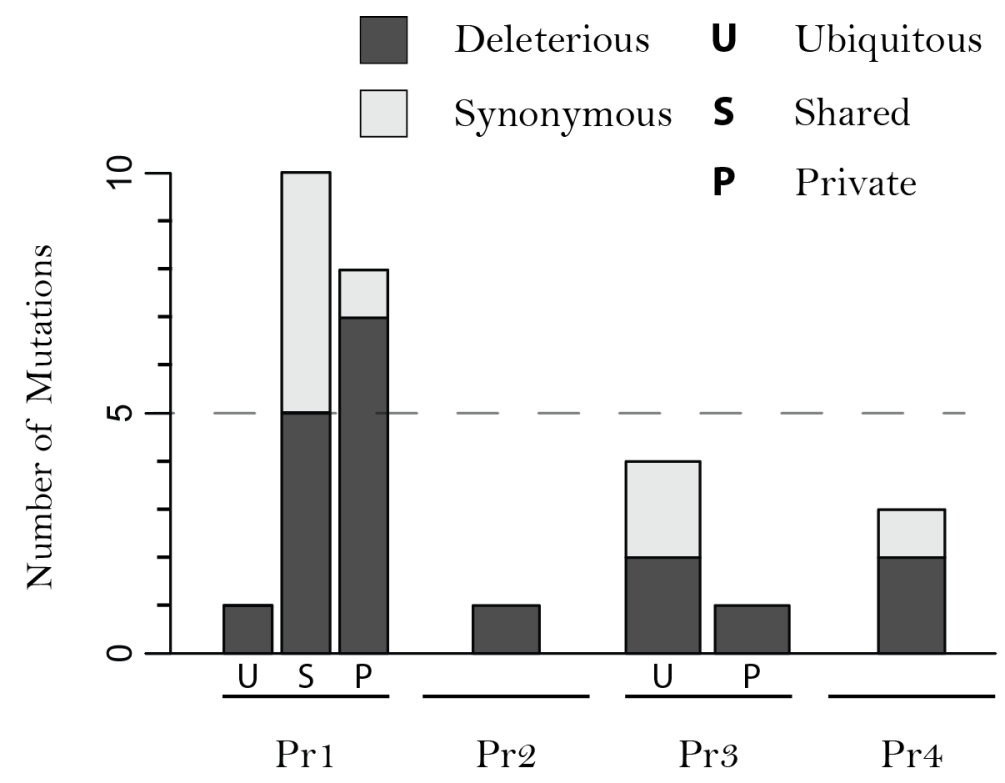

Figure 5.5 Consequence and distribution of mutations in synchronous PGL

Number and spatial distribution of coding mutations in primaries one through four as ubiquitous (seen in all regions), shared (seen in two or more but not all regions), and private (seen in only a single region). Primaries two and four were not multiregion sampled. The mutations have been further classified as deleterious, if they alter the protein sequence, or synonymous, if not.

therefore the synonymous mutation acts as a passenger during the enrichment of the deleterious mutation. Or alternatively it may suggest that mutations are not playing a large role in the early development of the tumour and are therefore not under great selective pressure. Interpretation of this is further complicated by the fact that not all deleterious mutations are advantageous and may themselves simply be passenger events. The uncertainty of using variant allele frequency to infer importance is highlighted by the finding that the two mutations present in the greatest proportion of cells in primary three are both synonymous, although there is emerging evidence of an active role for a subset of synonymous mutations (Supek et al., 2014), the majority are likely passenger events. It is perhaps the case that, beyond the primary driver mutation, early tumourigenesis is being driven largely by chromosomal aberrations, to which the mutations are acting as passengers. However, as tumour complexity increases and resources become less accessible, private mutations may provide incremental benefit to the tumour clone.

\subsubsection{Evolution of Primary One}

Multiregional sampling has been used successfully to map the evolutionary stages of several cancer types (Boutros et al., 2015; Gerlinger et al., 2014; Nik-Zainal et al., 2012). Here this same technique has been applied to primary one to develop an approximation of both the heterogeneity and timing of genomic 
alteration within a single tumour (Figure 5.6a and d). The ADTEx algorithm was used to derive copynumber profiles for each region of the tumour to assess the clonality of the changes detected through high-resolution SNP profiling. This analysis revealed that the loss of the short arm of chromosome 1 and loss of chromosome 18 were present in all cells in all regions of the tumour, suggesting they were ancestral events. In contrast, what had appeared to be a clonal loss of the long arm of chromosome 2 was in fact absent in region 1.2.3 and 1.2.4, and only present in a subset of the cells in region 1.2.2 (Figure 5.6b). The alteration was present in regions 1.1.1, 1.1.3, 1.2.1, and 1.2.2, suggesting that at least twothirds of the tumour harboured this alteration. Coupled with the observation that this loss event, although uncommon in PPGL, was also present in primary four suggests that in this patient the event may offer significant growth advantage to the clones in which it occurs.

Only a single mutation was found to be present in all regions sampled, a missense change in the vitamin $\mathrm{B}_{12}$ carrier-protein Transcobalamin II (TCN2). The specific amino acid change, located near the cterminal end of the cobolamin binding domain, has not previously been reported in the Catalogue of Somatic Mutations in Cancer (CoSMiC). Although vitamin $\mathrm{B}_{12}$ is a cofactor for several important enzymatic reactions, the activity of the TCN2 protein is largely cell extrinsic, where is facilitates the delivery and importation of vitamin B12 into the cell, after which the protein undergoes lysosomal degradation (Gherasim et al., 2013). Examination of chromosome 22 where the TCN2 gene is located did not demonstrate loss of the second allele, suggesting that the mutation would have to function either in a dominant negative manner or as a gain-of-function mutation to elicit an effect. Although, based on the RNA-seq expression data generated in Chapter Three, TCN2 is expressed in PPGL, given that the primary known activity for TCN2 occurs outside the cell, it is difficult to assess the effect of a mutation in intracellularly produced TCN2.

The PyClone algorithm, which utilises a Bayesian clustering method to model clonal population structures, was used to group the remaining variants into clonal populations. In cases where the algorithm appeared overly aggressive in grouping mutations, the output was manually refined. The analysis revealed mutational clusters representing ten distinct clonal populations within the tumour (Figure 5.6c). As mentioned previously, mutation Cluster A consisting of a mutation in TCN2 was the only mutation cluster present in all tumour regions. Mutation Cluster B was shared between regions 1.1.1, 1.1.3, 1.2.1, and 1.2.2, and appears to coincide with the population harbouring the loss of the long arm of chromosome 2. Mutation Cluster C was only present in regions 1.1.1 and 1.1.3. Mutation Cluster D represents a set of four private mutations found only in region 1.2.1 and likely represents an evolutionary branch distinct 
A

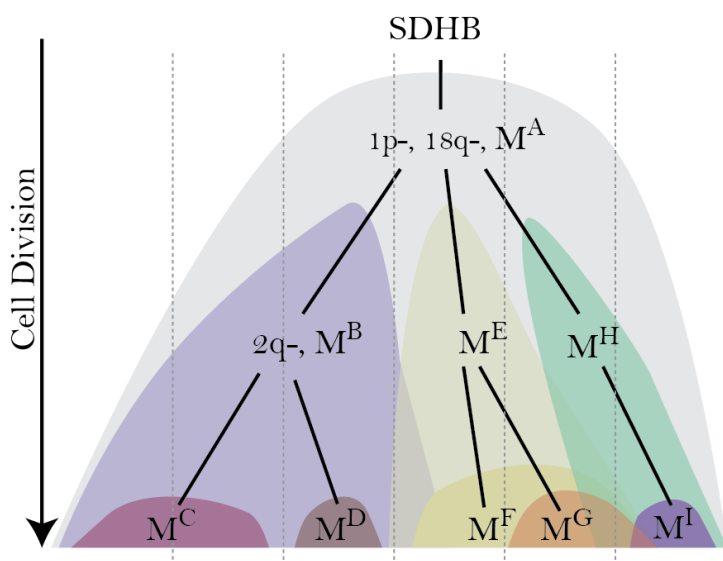

$\mathrm{B}$

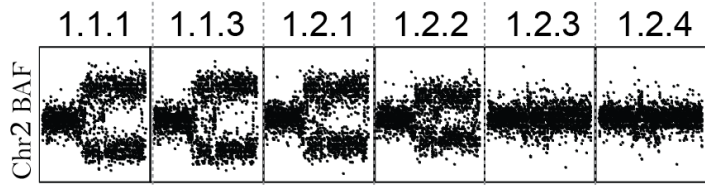

$\mathrm{D}$

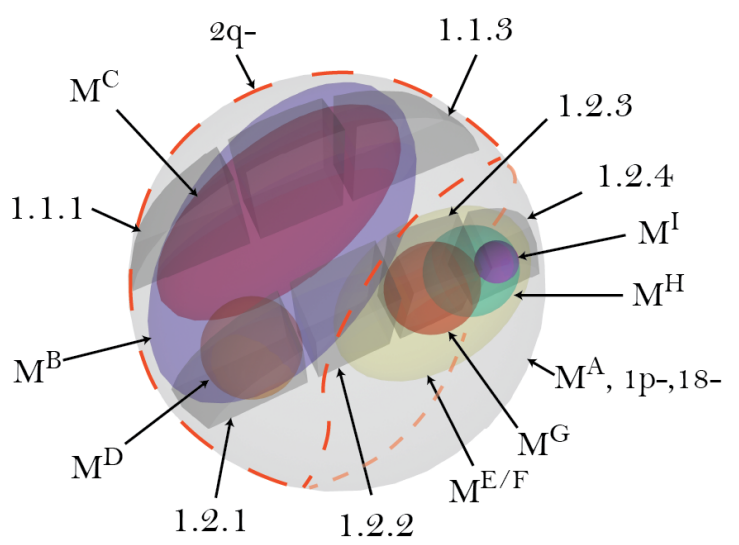

$\mathrm{C}$

\begin{tabular}{|c|c|c|c|c|c|}
\hline 0.07 & 0.16 & 0 & 0.03 & 0 & 0 \\
\hline 0.17 & 0.24 & 0 & 0 & 0 & 0.02 \\
\hline 0.09 & 0.12 & 0 & 0 & 0 & 0 \\
\hline 0.2 & 0.3 & 0.39 & 0.06 & 0 & 0 \\
\hline 0.24 & 0.3 & 0.26 & 0.18 & 0 & 0 \\
\hline 0.33 & 0.34 & 0.28 & 0.17 & 0.01 & 0 \\
\hline 0.28 & 0.26 & 0.28 & 0.18 & 0.01 & 0 \\
\hline 0.32 & 0.31 & 0.21 & 0.22 & 0.01 & 0 \\
\hline 0.33 & 0.36 & 0.27 & 0.06 & 0 & 0 \\
\hline 0.3 & 0.26 & 0.24 & 0.17 & 0 & 0 \\
\hline 0.26 & 0.23 & 0.25 & 0.15 & 0.01 & 0 \\
\hline 0.47 & 0.48 & 0.25 & 0.26 & 0.41 & 0.29 \\
\hline 0 & 0 & 0.13 & 0 & 0 & 0 \\
\hline 0 & 0 & 0.15 & 0 & 0 & 0 \\
\hline 0 & 0 & 0.2 & 0 & 0 & 0 \\
\hline 0 & 0 & 0.11 & 0 & 0 & 0 \\
\hline 0 & 0 & 0 & 0.06 & 0.13 & 0.09 \\
\hline 0 & 0 & 0.03 & 0 & 0.25 & 0.12 \\
\hline 0 & 0 & 0 & 0.2 & 0.42 & 0.15 \\
\hline 0 & 0 & 0 & 0 & 0.03 & 0.19 \\
\hline 0 & 0 & 0 & 0 & 0.05 & 0.15 \\
\hline 0 & 0 & 0 & 0 & 0.06 & 0.15 \\
\hline 0 & 0 & 0 & 0 & 0 & 0.11 \\
\hline 0 & 0 & 0 & 0 & 0 & 0.13 \\
\hline 0 & 0 & 0 & 0 & 0 & 0.14 \\
\hline
\end{tabular}

\begin{tabular}{|c|c|c|c|}
\hline $\begin{array}{l}\text { Gene } \\
\text { TMEM194B } \\
\text { IFFO1 } \\
\text { ASAP2 } \\
\text { NOTCH1 } \\
\text { IGKV1-8 } \\
\text { SLC17A9 } \\
\text { LGR6 } \\
\text { COL5A1 } \\
\text { ANKLE2 } \\
\text { PTPN2OB } \\
\text { FANCD2P2 } \\
\text { TCN2 } \\
\text { GBA3 } \\
\text { SAMSN1 } \\
\text { VPS 13D } \\
\text { FRYL } \\
\text { ZNF625-ZNF20 } \\
\text { TM4SF2 } \\
\text { FADS3 } \\
\text { RNTSKP116 } \\
\text { AXDND1 } \\
\text { RYR2 } \\
\text { ANGPT1 }\end{array}$ & $\begin{array}{l}\text { Consequence } \\
N S \\
N C \\
N S \\
\text { NS } \\
N C \\
N C \\
N C \\
N S \\
\text { S } \\
\text { SP } \\
N C \\
N S \\
N S \\
N S \\
N S \\
N C \\
N C \\
N C \\
N S \\
N C \\
N S \\
N C \\
N S\end{array}$ & $\begin{array}{l}\text { PyClone } \\
2 \\
2 \\
2 \\
2 \\
3 \\
3 \\
3 \\
3 \\
3 \\
3 \\
3 \\
3 \\
1 \\
4 \\
4 \\
4 \\
4 \\
5 \\
5 \\
5 \\
6 \\
6 \\
6 \\
6\end{array}$ & $\begin{array}{l}\text { Manual } \\
C \\
C \\
C \\
\text { B } \\
B \\
B \\
B \\
B \\
B \\
B \\
B \\
A \\
D \\
D \\
D \\
D \\
F \\
G \\
\text { E } \\
H \\
H \\
H\end{array}$ \\
\hline
\end{tabular}

Figure 5.6 Genomic evolution of primary one

(A) Schematic depicting the evolution of primary one through the accrual of sequential genomic alterations over time ( $y$-axis) to form the regions profiled ( $x$-axis). Copy-number alterations are denoted as chromosome number, arm, and event type (-,loss;+,gain). Clusters of mutation events are denoted with the letter $\mathrm{M}$ with a superscript annotation of the cluster as determined by PyClone and manual analysis (see panel C). (B) B-Allele frequencies for chromosome 2 across each region sampled. (C) Heatmap of regional VAFs for somatic mutations (rows) detected in any of the regions (columns) sampled. Mutation clusters assigned by the PyClone algorithm and manual refinement are given in the right hand columns. Mutation consequence is denoted as synonymous(S), non-synonymous(NS), non-coding (NC), or splice site (SP). (D) Spatial reconstruction showing relative positions of subclones within the tumour. Estimated area harbouring the loss of chromosome $2 q$ is marked with a dashed red line. 
from clones harbouring mutation set $\mathrm{C}$, both having arisen from a common ancestral clone harbouring mutations in Cluster B. Mutation Clusters E and F are present in sections 2.2.2, 2.2.3, and 2.2.4, however they have the highest VAF in region 2.2.3. This likely represents the subclonal centre for these populations, with the reduced VAF in adjacent regions demonstrating dilution through population admixture with surrounding subclones. Mutation clusters $\mathrm{G}$ and $\mathrm{H}$ are present in 2.2.3 and 2.2.4 in reciprocal proportions, likely representing spatial diffusion of two distinct subclones. Mutation cluster I is present only in 2.2.4, representing either an evolutionary descendant of the cells possessing cluster $\mathrm{H}$ mutations, or perhaps independent less fit branches from an ancestral clone. Fascinatingly, with the exception of the mutation in TCN2 and the loss of chromosome 18, regions 1.1.1 and region 1.2.4 are as genomically dissimilar to each other as they are to a region of any of the other synchronous primaries. There were no obvious instances of convergent branched chain evolution occurring within primary one as previously reported in $V H L$-driven RCC, although it is possible that some of the differentially mutated genes interact in pathways that are not yet fully characterised.

\subsection{Case Two: Multiple Endocrine Neoplasia Type IIA (MEN2A) 5.3.1 Patient Presentation}

A thirty-year old female presented with panic attacks and elevated metanephrine and normetanephrine levels. PET/CT imaging with ${ }^{18} \mathrm{~F}$-FDG revealed minimally avid hypodense right $(4 \mathrm{~cm})$ and left $(1 \mathrm{~cm})$ adrenal masses, as well as mildly avid right lung metastasis, a central right liver metastasis, and osseous metastases involving the pelvis, bilateral scapulae, humerii, and femora (Figure 5.7a). A region of intense tracer uptake was seen in the right lobe of the thyroid and nearby cervical lymph nodes, which was confirmed to be medullary thyroid carcinoma (MTC) via histological analysis of a tumour biopsy. PET/CT with GaTate demonstrated intense avidity in both adrenal masses but limited avidity in the thyroid, nodal metastases, and distant metastases (Figure 5.7b). SPECT/CT with ${ }^{123}$ I-MIBG mirrored the GaTate tracer pattern indicating that the metastatic disease likely originated with the MTC rather than the adrenal disease (Figure 5.7c). The patient underwent total thyroidectomy with reimplantation of parathyroid glands, resection of the cervical lymph nodes, and right adrenalectomy. Diagnostic sequencing revealed a germline mutation in RET (NP_066124:p.Cys634Tyr) consistent with a diagnosis of multiple endocrine neoplasia type $2 \mathrm{~A}$. 


\subsubsection{Specimen Collection and Genomic Profiling}

Fresh tissue specimens were obtained following surgical resection prior to fixation. A single region was obtained from the tumour mass present in the right thyroid (T1) (Figure 5.8a) and associated cervical lymph node metastasis (TM) (Figure 5.8b), and two regions representing each of the tumour poles were taken from the PCC (see Chapter 3 Figure 2). The remaining tissue was prepared for pathological review

A

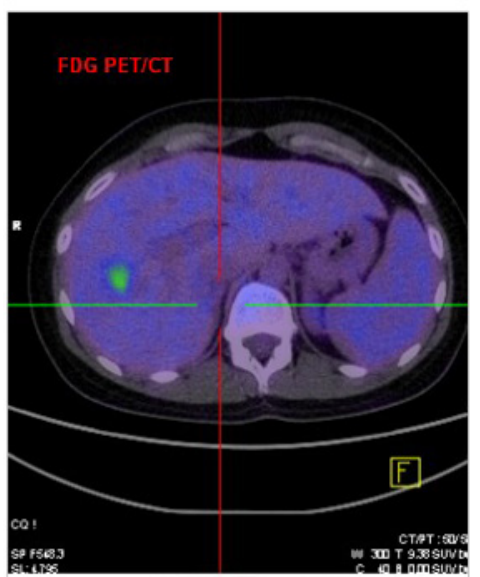

mases

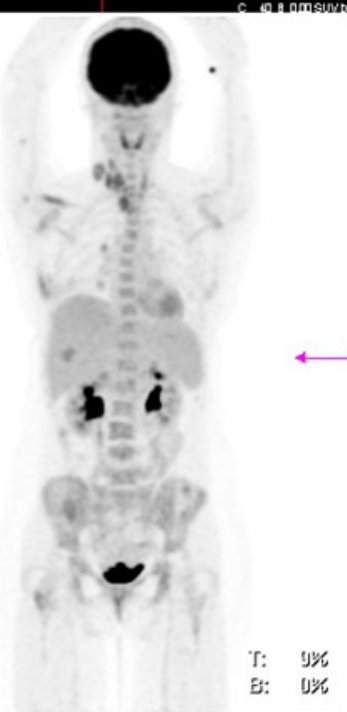

B

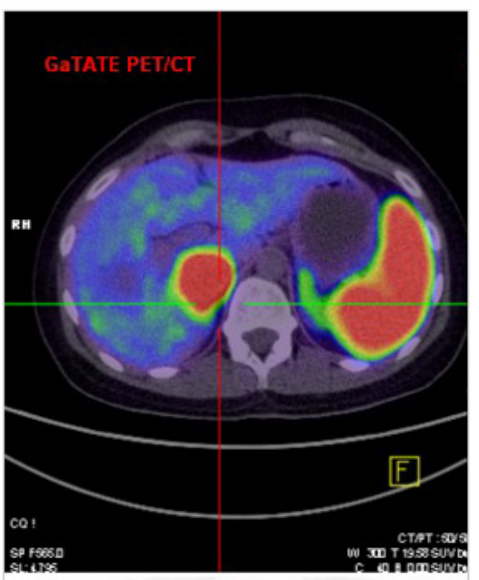

serescos

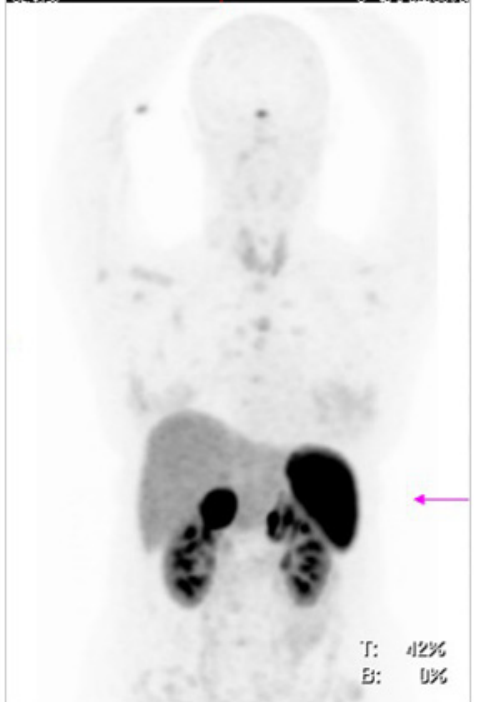

$\mathrm{C}$
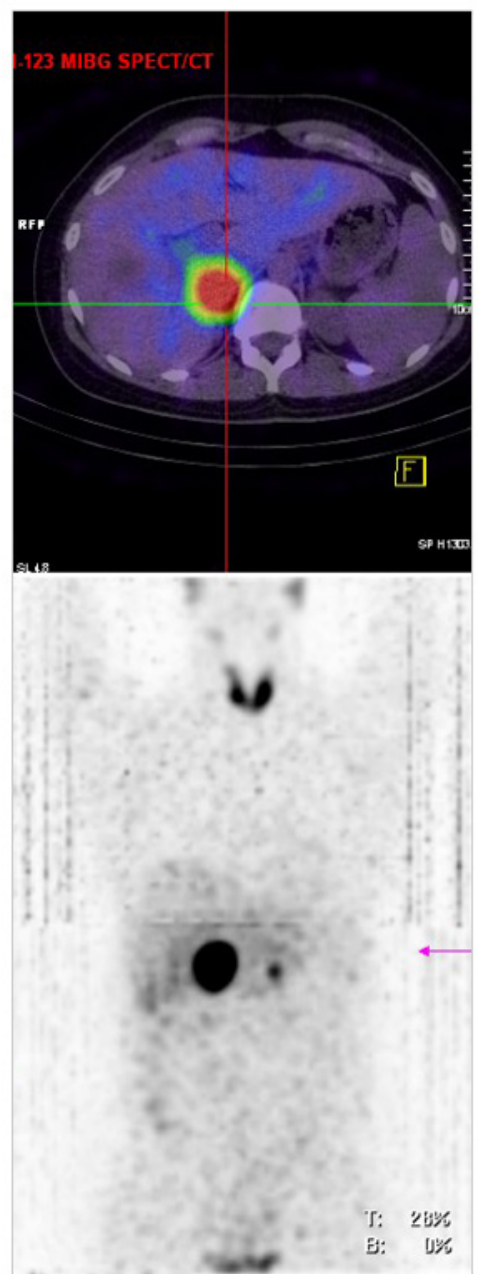

Figure 5.7 Molecular imaging of MTC and PCC

(A) 18F-FDG PET showing minimal tracer uptake in right and left adrenal masses, and moderate uptake in right lung, central right liver, and osseous metastases. A region of intense tracer uptake can be seen in the right lobe of the thyroid and nearby cervical lymph nodes.

(B) GaTate PET/CT and (C) 123I-MIBG SPECT/CT both demonstrated intense avidity in the adrenal masses with minimal to no avidity in the thyroid, nodal metastases, and distal metastases. 
by way of standard formalin-fixation and paraffin-embedding. Embedded specimens representing the right and left lobes of the thyroid gland were sectioned and stained with haemotoxylin and eosin or by immunohistochemistry for the cellular proliferation marker Ki-67. Review of Ki-67 staining of the right lobe of the thyroid revealed distinctive regions of high and low of Ki-67 expression (Figure 5.8c). As Ki67 expression has been shown to clinically correlate with metastatic disease (Li et al., 2015), a region of high (T1Ki67) and low (T2) expression were selected to assess which was most genomically similar to TM. Staining of sections obtained from the left lobe of the thyroid revealed a small region of dysplastic growth previously undetected by molecular imaging (Figure 5.8d). Genomic profiling was also performed on this region (T3) to determine if it represented a metastatic deposit or a synchronous primary tumour. Circulating nucleic acids (cfDNA) were isolated from peripheral whole blood taken prior to surgery (see Methods 2.2.2.5). Exome sequencing was performed on all fresh-frozen, formalin-fixed, and cfDNA samples using the SureSelect V5 target enrichment platform, high-resolution copy-number profiling was performed using the CytoScan HD or OncoScan FFPE assay on fresh frozen and FFPE samples respectively. RNA-seq libraries were generated for T1, TM, and both PCC regions using the Illumina TruSeq RNA V2 sample preparation kit (see Methods 2.2.4.1).

\subsubsection{Genomic Alterations in Synchronous MTC and PCC}

\subsubsection{MTC}

Copy-number profiling revealed loss of the short arm of chromosome 1 in all samples taken from the thyroid (Figure 5.9a). In the T1, T1Ki67, and TM, the breakpoint was found to be approximately $13 \mathrm{Mb}$ upstream of the centromere, while samples T2 and T3 were found to have centromeric break points (Figure 5.9b). The distinct break points indicate that T2, the low Ki67 region sampled from within the right lobe primary tumour, and T3, the dysplastic region taken from the left lobe are in fact independent of T1, and originate from distinct tumourigenic events. T1, T1Ki67, and TM, all demonstrated gain of the regions downstream to the breakpoint on chromosome 1, suggesting that both the gain and loss events resulted from the same rearrangement, and providing additional confirmation that $\mathrm{T} 1$ was the site of origin for the parent clone of TM $\left(\mathrm{TM}_{\mathrm{P}}\right)$. The limited literature available regarding copy-number alterations in MTC suggests that loss of the short arm of chromosome 1 is a frequent occurrence, older publications report frequencies of between 21 and 30\% (Marsh et al., 2003; Ye et al., 2008), while a more recent publication demonstrated frequencies of up to $47 \%$ in sporadic cases harbouring a $R E T$ mutation and $37 \%$ overall. To assess whether the ubiquity of chromosome $1 \mathrm{p}$ loss in this patient may be an indication of a cooperative germline mutation, allele enrichment analysis was used to determine the set of alleles lost in each sample (Figure 5.10). 


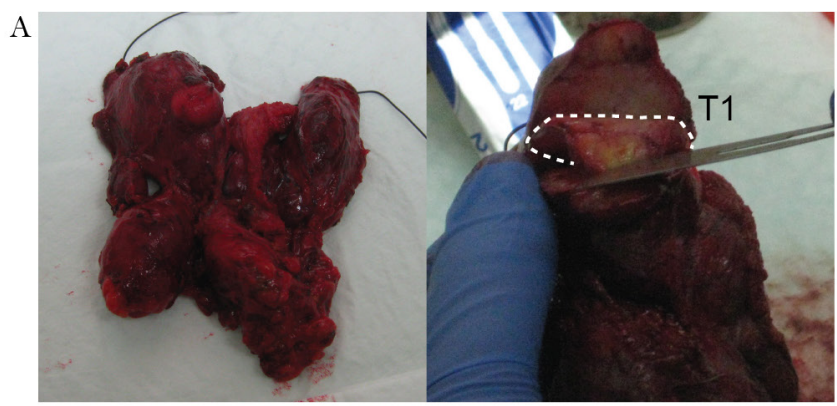

$\mathrm{B}$
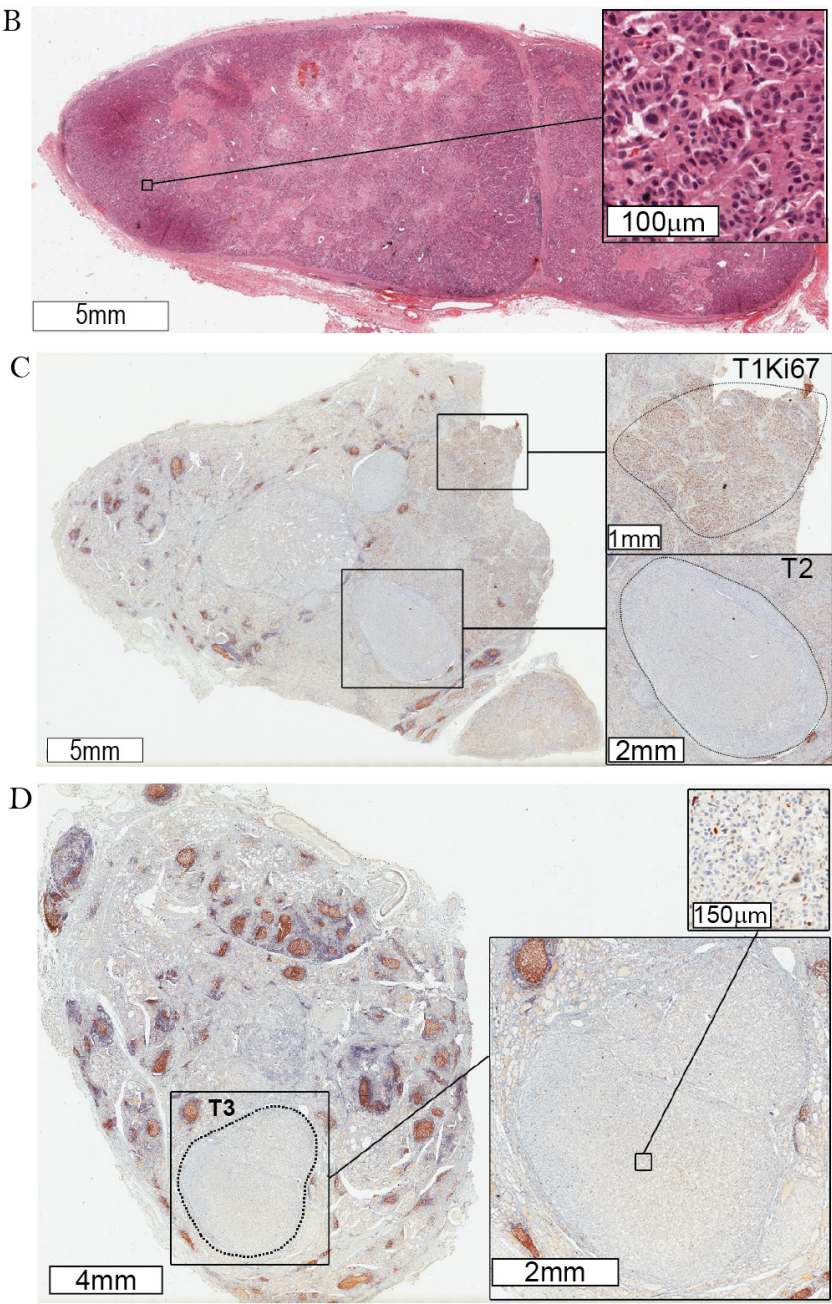

Figure 5.8 Medullary thyroid carcinoma biospecimen collection

(A) Thyroid gland following surgical resection. The sliver of tissue marked by a white outline was taken from the right thyroid and snap frozen for genomic profiling (specimen T1). (B) FFPE section of cervical node stained with haematoxylin and eosin. Specimen TM was obtained as fresh frozen tissue from an alternate node. (C) FFPE section of right thyroid tumour stained against Ki-67. The region of high Ki-67 (specimen T1Ki67), and low Ki-67 (specimen T2) used for genomic profiling are marked with black dotted outlines. (D) FFPE section of left thyroid stained against Ki-67. The region extracted for genomic profiling is marked by dotted outline (specimen T3). 

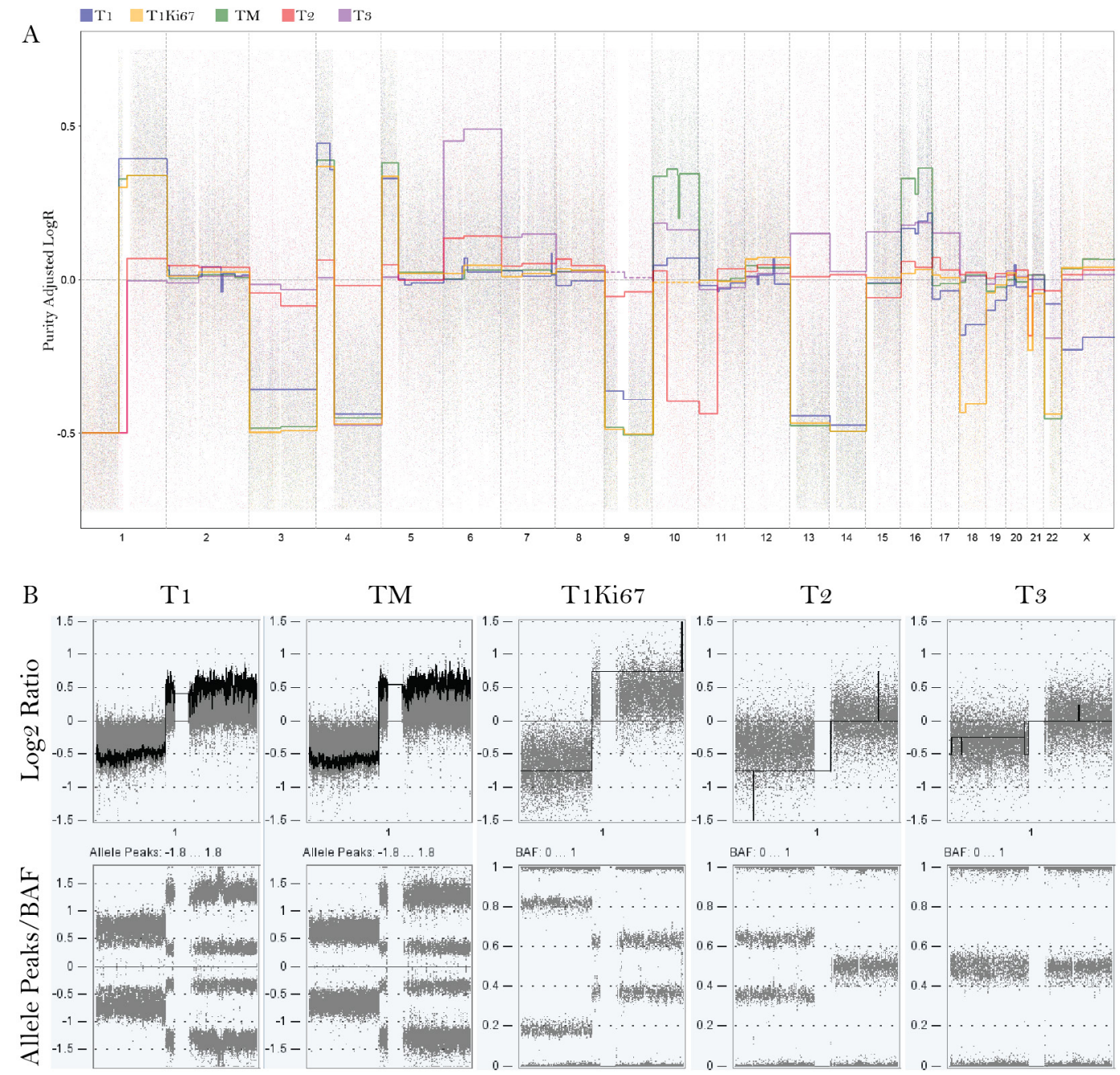

Figure 5.9 High resolution copy-number analysis of MTC

(A) Overlay of high resolution copy-number analysis of T1, T1Ki67, TM, T2, and T3. Data smoothing was performed using the aspcf algorithm in the copynumber package for R. Utilising the assumption that loss of $1 p$ is an early event, sample purity and array variance were normalised by multiplying probe and smoothened values by a scaling factor to establish a mean probe value of -0.5 on chromosome $1 p$ in each sample. (B) High resolution copy-number analysis demonstrating shared non-centromeric chromosome $1 \mathrm{p}$ breakpoints in T1, T1Ki67, and TM, as distinct from centromeric break points seen in T2 and T3.

As expected T1, T1Ki67, and TM had lost concordant copies of chromosome 1p, however, T2 had lost the complementary set of alleles. Unfortunately the sample purity obtained for T3 rendered the analysis for this sample inconclusive. This suggests that the loss of chromosome $1 \mathrm{p}$ is not enriching an existing deleterious genome feature and that the oncogenic contribution of this event is likely one of compound haploinsufficiency. Gain of the short arm of chromosome 4 as well as loss of chromosomes 13, 14, and 
the long arm of chromosome 4 were present in T1, T1Ki67, and TM at a frequency similar to the loss of the short arm of chromosome 1. Analysis of allele enrichment demonstrated loss of the same allele set in all samples suggesting that these were ancestral events, which is concordant with the high clonal frequency (Figure 5.10). Chromosomes 3, 9, and 22 were also lost at a similar frequency to chromosome $1 \mathrm{p}$ in T1Ki67 and TM, however the loss of chromosome 3 and 9 were present in a marginally reduced proportion of cells in $\mathrm{T} 1$ and the loss of chromosome 22 was absent. Initial interpretation would suggest that loss of chromosomes 3 and 9 were early events common to a large proportion of cells including the T1Ki67 and $\mathrm{TM}_{\mathrm{P}}$ clones, while loss of chromosome 22 was a later event occurring in a more recent ancestor of T1Ki67 and $\mathrm{TM}_{\mathrm{P}}$. However, analysis of the enriched alleles demonstrated that T1Ki67 and TM had lost opposite copies of both chromosomes 3 and 22, suggesting that these loss events occurred independently after these clones had diverged from one another. While this may also be the case for chromosome 9, allele enrichment demonstrated loss of the same copy in both clones. As such, it is not possible to determine the timing of this event. Loss of chromosomes 22 and the long arm of chromosome 4 are also detectable in T3, interestingly, chromosome 9 had undergone copy neutral loss of heterozygosity $(\mathrm{CNLOH})$ in this tumour. It is difficult to interpret the role of CNLOH as the risk of haploinsufficiency is greatly reduced. Examination of the allele enrichment profile demonstrated that the copy retained matched that of T1Ki67 and TM (Figure 5.10), so it is plausible the alterations to chromosome 9 are enriching a deleterious mutation or abrogating the active copy of an imprinted gene (e.g. GLIS3), as despite alterations to chromosome 9 having been previously reported as a feature of MTC (Marsh et al., 2003), they do not appear to be common (Flicker et al., 2012; Ye et al., 2008). Together the alterations of chromosomes 3, 9, and 22 provide striking examples of convergent evolution during the development of MTC, unfortunately, as was seen in PPGL, the alterations involve large chromosomal regions making it difficult to infer the critical genes involved.

Despite these examples of convergence T1, T2, and T3, display quite distinct copy-number profiles. T1 has experienced six chromosomal loss and four gain events, while T3 demonstrates three loss events, seven gain events, and a CNLOH event. In contrast, T2 has a comparatively stable genome exhibiting only three loss events and no gains. While this could suggest an inherent difference in the genomic stability between T2 and the other tumours, it is more likely a reflection of the number of cellular divisions since tumour incipience. However, with that in mind, it is interesting to note that TM does not exhibit any copy-number alterations that are not detectable in T1, nor does the nodal metastasis demonstrate any alterations at subclonal frequency, a surprising observation given that the clone would have undergone significant expansion following metastatic seeding. This suggests that the copy-number 


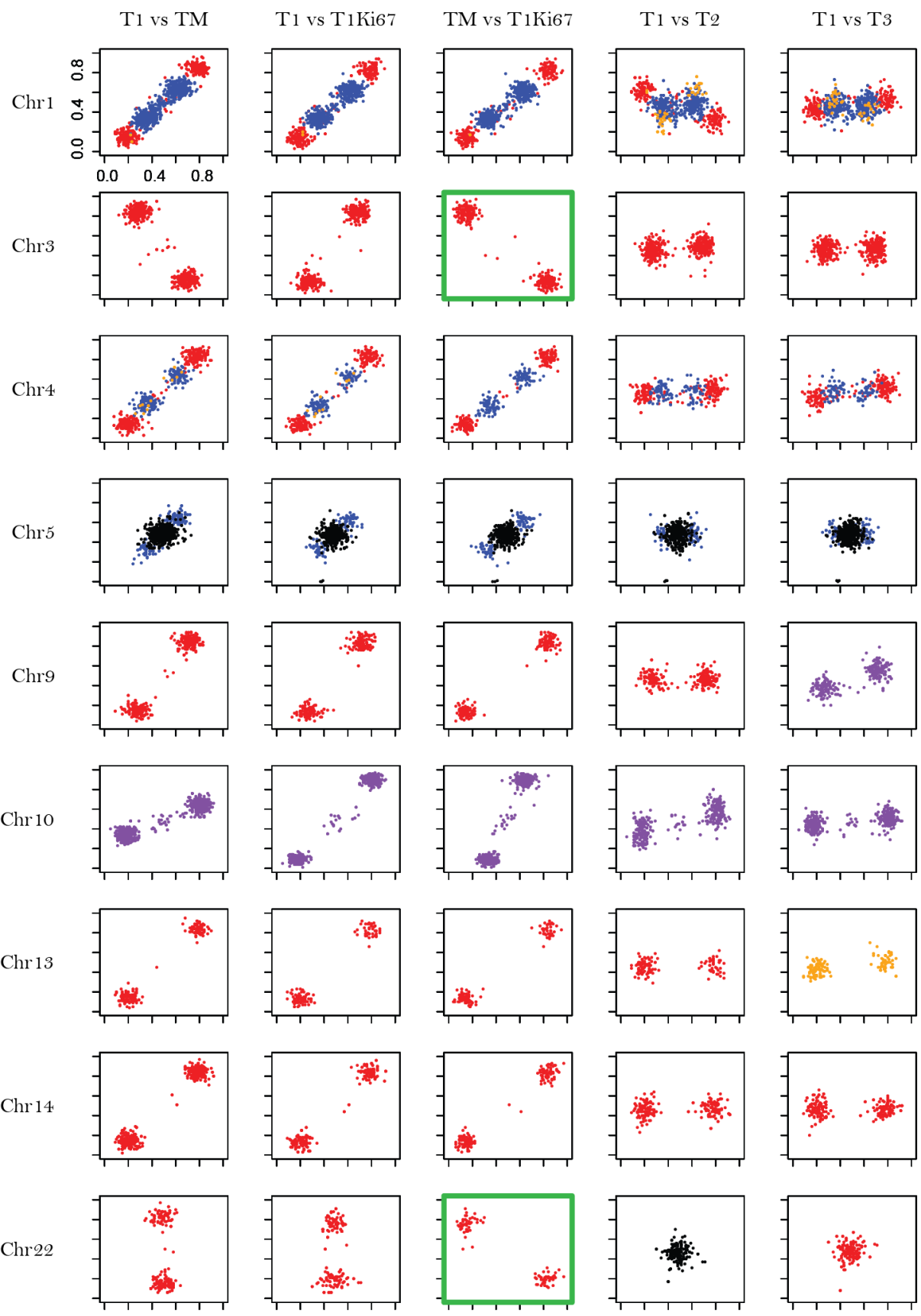

Figure 5.10 Allelic enrichment analysis

Correlation of alleles enriched by CNA. Germline SNPs were filtered to retain those with a read-depth greater than 50 in the tumour and germline, a VAF between 0.45 and 0.55 in the germline, and a dbSNP identifier. Plots show the corresponding VAF ( $x$ and $y$ axis) for each SNP in each pair of samples. Data points are colour coded to indicate SNPs in regions of loss (red), gain (blue), $\mathrm{CNLOH}$ (purple), and opposing loss/gain (orange). Positive correlations indicate identical allele sets (i.e. chromosomal copies) enriched, while negative indicates opposing sets. Horizontal or vertical diametric clusters indicate normal copy-number in one sample, while a central cluster indicates the absence of a copy number alteration. Suspected convergence events within the T1Ki67 and TM subclones are highlighted with a green border. Low sample purity obfuscates most comparisons for T3. 


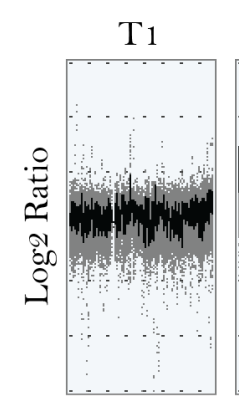

10

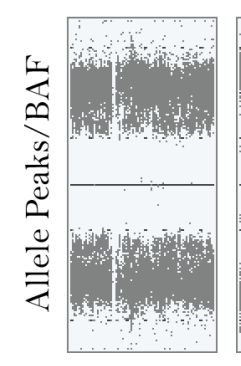

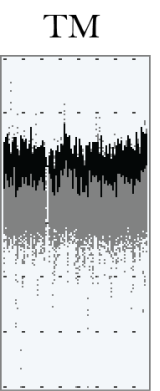

10

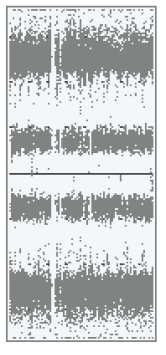

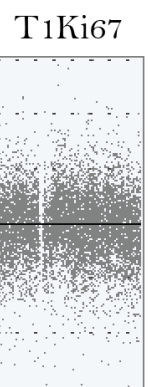

10

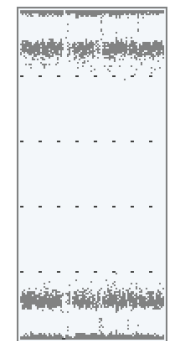

$\mathrm{T} 2$

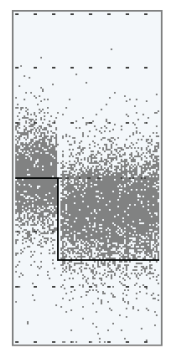

10

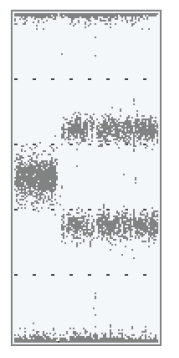

T3

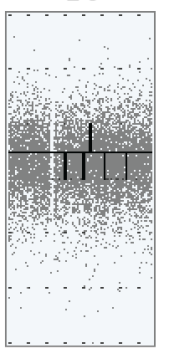

10

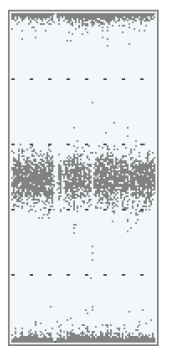

Figure 5.11 Chromosome 10 copy-number alterations

High-resolution copy-number analysis showing the copy state of the RET gene in each MTC sample. Single copy loss is seen in T2, while copy neutral LOH can been seen in T1Ki67. TM and T3 exhibit a single copy gain. T1 demonstrates a complex copy state resulting from a mixture of trisomy 10 in the TMP clone, seen as an elevated log 2 ratio, and copy neutral LOH in T1Ki67, seen as a diametric B-allele frequency shift.

alterations present in the $\mathrm{TM}_{\mathrm{P}}$ departing $\mathrm{T} 1$ may represent a kind of evolutionary peak given the selective pressures being applied to the tumour. It is interesting to speculate on whether given enough time T2 and T3 would have approached the challenges of metastasis by ultimately resembling TM, or by finding a unique but equally efficient evolutionary peak.

Allelic imbalance events involving the RET gene have been shown to be common in RET-driven PCC and MTC (Ciampi et al., 2012; Huang et al., 2000; Koch et al., 2001). Examination of copy-number data for this case revealed several patterns of chromosome 10 alteration (Figure 5.11). T1Ki67 exhibited CNLOH resulting from loss of the wild-type copy and gain of the copy harbouring the RET mutation, while TM demonstrated trisomy ten having gained an additional copy of the mutant allele. T1 demonstrated a similar B-allele frequency pattern to T1Ki67, however, the $\log \mathrm{R}$ values showed an elevated signal consistent with the contribution of trisomy 10 in $\mathrm{TM}_{\mathrm{P}}$. These data suggest that the majority of cells present in $\mathrm{T} 1$ had experienced enrichment of the RET mutation through CNLOH and that trisomy 10 was restricted to a minor portion of cells. This is particularly interesting when one considers the order of acquisition of genomic changes. Alterations to chromosomes 1, 4, 13, and 14, are common to T1Ki67 


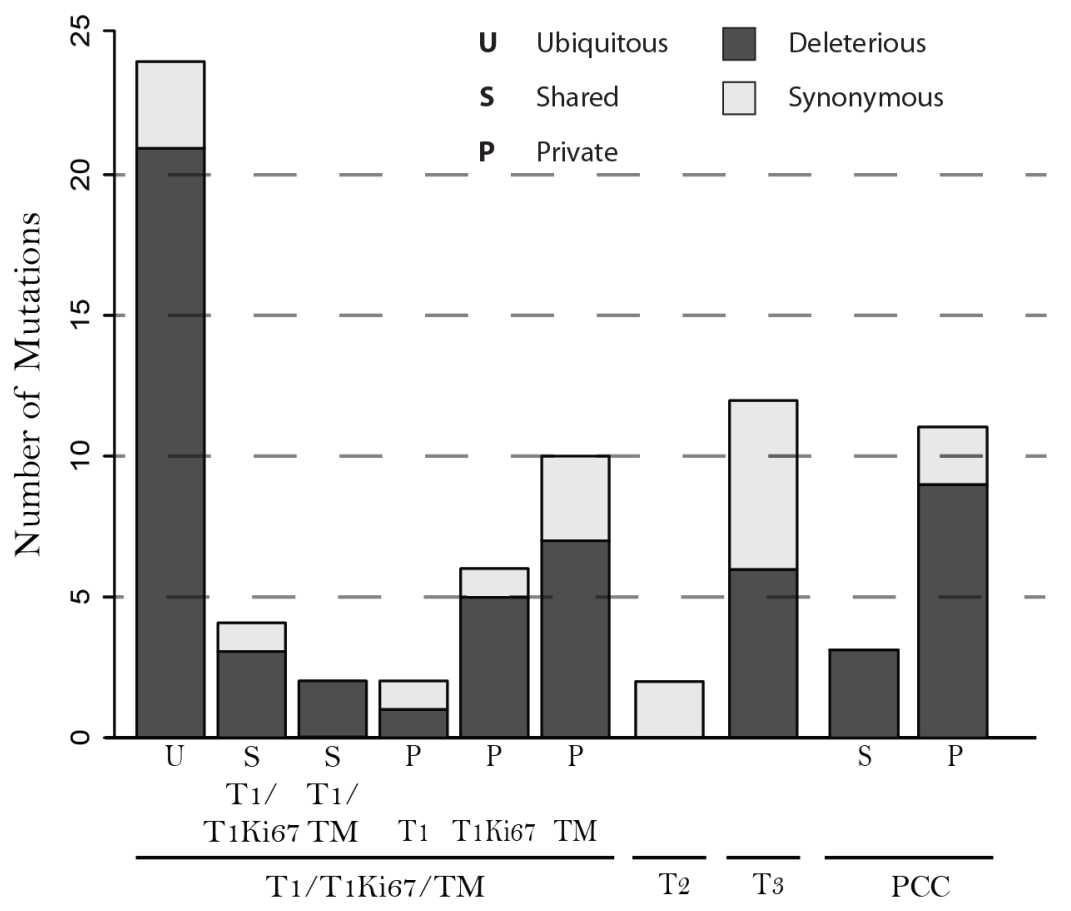

Figure 5.12 Consequence and distribution of mutations in synchronous MTC and PCC (Case 2)

Number and spatial distribution of coding mutations in MTC and PCC as ubiquitous (seen in all regions), shared (seen in two or more but not all regions), and private (seen in only a single region). Shared mutations in T1 have been subdivided to indicate whether they were shared with T1Ki67 or TM. The mutations have been further classified as deleterious, if they alter the protein sequence, or synonymous, if not.

and $\mathrm{TM}_{\mathrm{P}}$ suggesting that these clones shared a common ancestor, in order for the wild-type copy of chromosome 10 to be present in TM it must have been retained throughout the shared history of these clones. The observation that CNLOH is the dominant state within T1 suggests that the loss of the wildtype copy has also occurred independently within clones divergent from T1Ki67 and T1M wild-type copy is also seen in T2, however there has not been a gain of the mutant copy, while T3 demonstrates trisomy having undergone a gain of the mutant copy. Enrichment of the mutant allele can be seen as the common factor in each of these events, either through gain of the mutant copy, loss of the wild-type copy, or a combination of both leading to CNLOH. It is unclear whether duplicate mutant alleles through trisomy ten or $\mathrm{CNLOH}$ provides the greater growth advantage, although given the oncogenic nature of $R E T$, it seems intuitive to assume that more copies would provide the greater advantage. As such, the convergence towards the $\mathrm{CNLOH}$ state seen in $\mathrm{T} 1$ may represent a trade-off designed to alleviate gene dosage issues caused by trisomy while maintaining maximum oncogenic activation. There is also the possibility that wild-type RET provides some level of negative feedback, and thus, even in the absence of amplification of the mutant, it is advantageous to remove the wild-type allele, 
as was seen in T2. Without a greater understanding of the dynamics of the wild-type-mutant relationship as well as the effects of trisomy ten on gene dosage, it is difficult to speculate on how trisomy ten contributed to the metastatic potential of $\mathrm{TM}_{\mathrm{P}}$. Previous attempts to utilise RET copy-number alteration as a prognostic factor in MTC have been inconclusive (Ciampi et al., 2012).

The mutational landscape of T1, T2, and T3 mirrors the respective complexity of their copy-number profiles. T1 had the largest number of mutations with thirty-two detectable coding region mutations, followed by T3 and T2 with twelve and two coding region mutations, respectively (Figure 5.12). Twentyfour of the mutations present in T1 could also be detected in both T1Ki67 and TM, T1 and TM shared two mutations absent in T1Ki67, while T1 and T1Ki67 shared four mutations absent in TM (Figure 5.13). The highest variant allele frequencies among the shared mutations were observed for missense mutations in the genes FCAMR and MAX. Taking into account the location of FCAMR on the long arm of chromosome 1, the high VAF likely indicates that the mutation was present prior to the copy gain event, suggesting that it may either be a very early oncogenic event or a passenger mutation present in the pretransformed cell. A previous exome study described a single case with a frame shift deletion in FCAMR (Agrawal et al., 2013), however, as FCAMR is primarily expressed in lymphoid tissues where it facilitates endocytosis of IgM and IgA antibodies (Sakamoto et al., 2001), it is unclear what advantage a mutation might offer the developing MTC. The role of MAX mutations in the development of PPGL has been previously discussed, however, there is mounting evidence for a more general role for MAX in neuroendocrine tumour biology. A recent study in small cell lung cancer (SCLC) encompassing fiftythree cell lines and forty-five primary tumours found MAX-inactivating alterations in $6 \%$ of SCLCs tested (Romero et al., 2014). In addition, the study detected mutations in BRG1, a regulator of $M A X$ expression, that were mutually exclusive with mutations in $M A X$. Although the particular missense mutation present in this case has not been reported in COSMIC, analysis with the mutation effect prediction algorithms, SIFT, PolyPhen, and Condel, unanimously predicted the variant to have a damaging effect on protein function. Furthermore, loss of chromosome 14 has resulted in abrogation of the remaining functional copy. Together these findings strongly support $M A X$ as a potential driver in MTC.

T1 harboured just two mutations not detectable in T1Ki67 or TM suggesting the tumour had undergone periods of linear evolution, a process marked by the outgrowth of a single dominant clone following each beneficial genomic alteration (Figure 5.14). T1Ki67 harboured six mutations not present in either T1 or TM, these likely represent branched evolution occurring locally within the high Ki67 region which was 

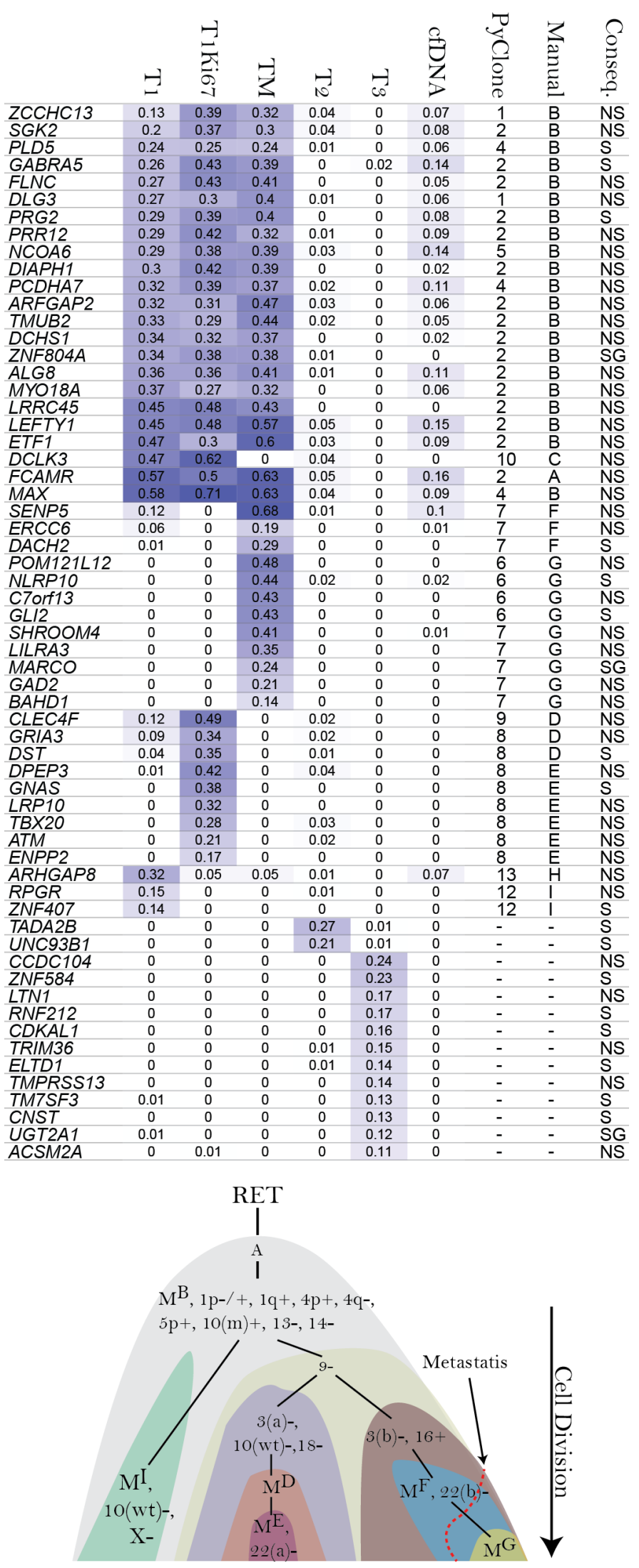

Figure 5.13 Shared and private variants in MTC Heatmap showing VAFs for somatic mutations (rows) detected in any MTC specimen (columns). Values in the PyClone and Manual columns indicate the cluster each mutation was assigned by the respective method. Variants shared between $\mathrm{T} 2$ and $\mathrm{T} 1$ are likely the result of sample contamination with peripheral T1 cells during macrodissection. Mutation consequence is denoted as synonymous (S), nonsynonymous (NS), or stop gained (SG).
Figure 5.14 Evolution of T1, TiK67, and TM

Schematic depicting the evolution of various subclones ( $x$-axis) through the sequential accrual of genomic alterations over time ( $y$-axis). Copy-number alterations are denoted as chromosome number, arm, and event type (-,loss; +,gain). Clusters of mutation events are denoted with the letter $M$ with a superscript annotation of the cluster as determined by PyClone with manual adjustment (see Figure 5.10). Values in brackets indicate which chromosomal copy was lost as mutant(m), wildtype(wt), and allele set A/B. 
either not sampled as part of $\mathrm{T} 1$ or represented an insufficient fraction for the variants to be detected. Of particular interest is a missense change in the DNA damage checkpoint modulator ATM (NP_000042.3:p.Val2152Glu), loss of which has been shown to significantly increase the proliferative index of intestinal neoplasia in mouse models (Kwong et al., 2008). The same study that described FCAMR mutations also described ATM and MDC1 mutations in RET-mutation negative MTC, leading the authors to suggest a potential role DNA damage pathways in these cases (Agrawal et al., 2013). However, the current data suggest that ATM mutations may enhance the proliferative rate of MTC regardless of the

primary driver mutation. TM harboured three synonymous and seven deleterious private mutations which likely arose after $\mathrm{TM}_{\mathrm{P}}$ metastasised from $\mathrm{T}$, although it is possible some were present in $\mathrm{T} 1 \mathrm{at}$ subdetectable levels. Examination of RNA-seq data for the seven deleteriously mutated genes revealed that three were not expressed within limits of detection, while one $\mathrm{x}$-linked mutation could not be detected in the RNA-seq reads, possibly due to selective inactivation of the mutant allele through $\mathrm{x}$-inactivation. These data suggest that although TM continued to accrue mutations after metastasis, the majority appear not to contribute to the metastatic phenotype, further supporting the idea that $\mathrm{TM}_{\mathrm{P}}$ represents a stable evolutionary peak given the environmental pressures. Another possibility is that point mutations in general play a very minor role in early MTC oncogenesis, an idea supported by the observation that only two of the coding mutations to pass filtering in T2 were synonymous changes, as were six of the twelve mutations detected in T3. In this regard, MTC may mirror what was seen for PPGL driven by loss of $S D H B$ whereby, subsequent to the initial driver mutation, early oncogenesis appears to be largely driven by copy-number alterations and the resulting compound haploinsufficiency of multiple tumour suppressor genes.

\subsubsection{PCC}

A brief description of the genomic changes detected in the poles of the PCC was included as part of the publication in Chapter Three (see Chapter 3 Figure 2) and will be covered in more detail here.

Copy-number profiling demonstrated losses of chromosome 1p, chromosome 3 downstream of $3 \mathrm{p} 14.3$, chromosome 9 between 9q21.11 and 9q31.3, chromosome 10 between 10q23.1 and 10q25.1, chromosome 14 from 14q32.2, and chromosome $17 \mathrm{p}$ in both poles of the tumour. Interestingly, the alteration to chromosome 10 does not encompass the RET gene, which remains balanced diploid. An additional loss of chromosome 21 was detected in the left pole, while the right pole demonstrated a $0.38 \mathrm{Mb}$ segmental gain encompassing the genes YIPF5 and KCTD16, which are involved in the maintenance of Golgi structure (Yoshida et al., 2008) and GABA(B) receptor modulation (Metz et al., 2011), respectively. The signal ratio for the loss of chromosome 21 is of a lesser magnitude comparative 
to the other copy-number alterations, suggesting that although dominant, it is not present in all cells in the region. This cellular composition may indicate either that the loss was a recent event occurring locally within the region which imbued a significant growth advantage, or an earlier event which gave only minor advantage allowing other clones to grow in parallel, sampling of additional regions would be required for clarification.

A total of fourteen coding region mutations, two synonymous and twelve deleterious, were detected across both poles combined, however, only three missense changes were ubiquitous (Figure 5.12). Only one of the three ubiquitous mutations could be detected as expressed by RNA-seq; a missense change in the cellular trans-Golgi network (TGN) membrane protein, GOLGA1, which is involved in trafficking between the TGN and endosomal system (Jing et al., 2010; Lock et al., 2005). A shared mutation in $N R K$, a member germinal center kinase family shown to be predominantly expressed in skeletal muscle during the late stages of mouse embryogenesis (Kakinuma et al., 2005), was not detectable by RNA-seq, as NRK is located on chromosome $\mathrm{X}$ it is possible that the variant is located on the inactive copy. The final ubiquitous mutation, located in the unprocessed pseudogene $P C D H B 17$, is difficult to interpret, unlike mutations in coding genes there is no framework available for assessing mutations in non-coding RNA, despite emerging evidence of their critical role in complex gene regulation networks (Rinn \& Chang, 2012). The left pole harboured two synonymous and five missense private mutations, while the right pole demonstrated four private missense mutations. The majority of private mutations were present at a high VAF reflecting the purity of the respective copy-number alterations in each pole.

It seems likely that the tumour underwent an extended period of linear evolution in which the shared copy-number alterations and mutations were established, after which one or more branching events occurred that were then followed by further linear evolution along each branch. The high number of shared copy-number alterations relative to shared mutations again supports a strong role for loss of tumour suppressor genes in early PPGL development.

\subsubsection{3 cfDNA}

Deeper understanding of tumour heterogeneity combined with the availability of increasingly sensitive massively parallel sequencing methods has created a surge in interest in the diagnostic utilisation of circulating nucleic acids. Circulating DNA, or cell-free DNA (cfDNA), is a term given to short fragments of DNA released into the blood stream from apoptotic or necrotic cells which have escaped phagocytosis by scavenger cells (Schwarzenbach et al., 2011). At present the is no literature examining the detection of specific mutations in cfDNA derived from MTC or PCC, and only a single study on the potential use of 
cfDNA abundance as a diagnostic test in MTC (Zane et al., 2013). In light of this, exome sequencing was performed on cfDNA isolated prior to surgery to provide an initial insight into the relative representation of ubiquitous, shared, and private variants in the cfDNA. As variant calling pipelines may be less effective at low VAFs typical of cfDNA, additional manual inspection of alignment data was performed for all variant bases detected in any other specimen. In total, twenty-eight variants were detected in the cfDNA, twenty-two of these were ubiquitous amongst T1, T1Ki67, and TM, two were shared between T1 and TM but absent in T1Ki67, two were private to TM, and two were unique to the cfDNA. Excepting two mutations with poor sequence coverage, all ubiquitous mutations were detectable in the cfDNA. Unsurprisingly, the highest detected VAF in the cfDNA belonged to the FCAMR mutation, reflecting the combination of an ancestral event and allelic amplification. In contrast, the VAF of the remaining variants in the cfDNA showed very little correlation with their VAF in either T1 or TM $\left(\mathrm{R}^{2}: 0.18\right.$ and 0.17 , respectively). The high degree of variation in the VAFs detected in the cfDNA relative to the VAF in the parent tumour is more likely a limitation of the sample preparation process rather than a biological phenomenon. However, a second technique such as amplicon sequencing would be required for confirmation. No mutations that were private to T1, T1Ki67, T2, or T3 were detectable in the cfDNA. Furthermore, a mutation in DCLK3, located on chromosome 3, which was present at high VAF in T1 and T1Ki67 but absent in TM, probably due to loss of the mutant copy, was not detectable in the cfDNA despite sufficient coverage (read-depth: 96). These data suggest that the majority of cfDNA detected was being released from one or more metastatic sites either seeded by $\mathrm{TM}_{\mathrm{P}}$ in parallel with $\mathrm{TM}$ or reseeded by TM subsequent to establishment. As such, mutations in TRIM46 and ZNF696 detected exclusively in the cfDNA may have originated at other metastatic sites. Interestingly, only two of the mutations present in the PCC were detectable in the cfDNA and both in only a single read, representing less than $1 \%$ of the total cfDNA reads. It is likely this disparity is correlated with disease burden (Diehl et al., 2008), however, as various cancer types have been shown to emit differing levels of cfDNA, it may represent a difference between the biology of PCC and MTC (Bettegowda et al., 2014).

\subsubsection{Common Alterations in PCC and MTC}

There are currently over 100 genes thought to contribute to cancer susceptibility when mutated in the germline (Rahman, 2014). One of the most fascinating and puzzling aspects of cancer susceptibility genes (CSG) is that, despite many of them having central roles in cellular biology, they often contribute to tumour formation only within a small array of tissue types. A possible implication of this is that the particular tissues affected by a CSG may share common biochemical pathways with respect to the 


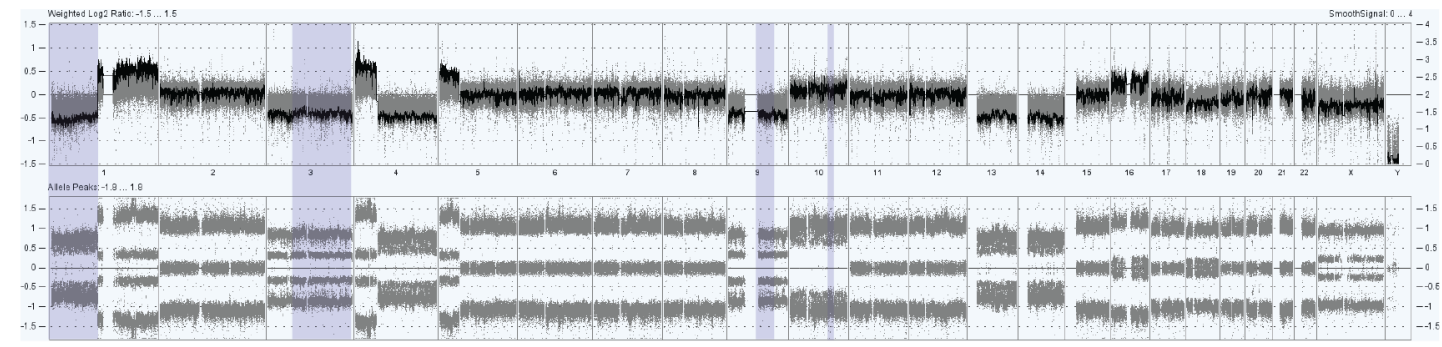

PCC-LP

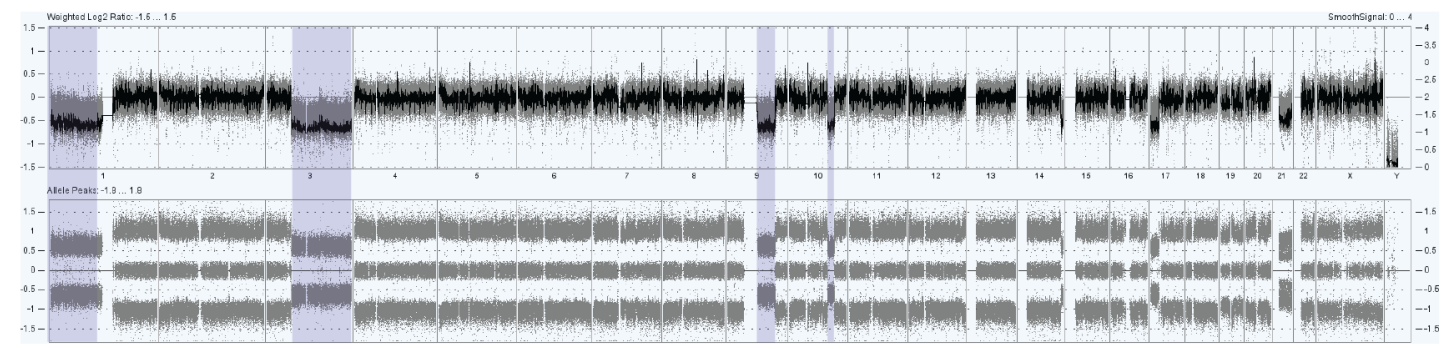

$\mathbf{B}$
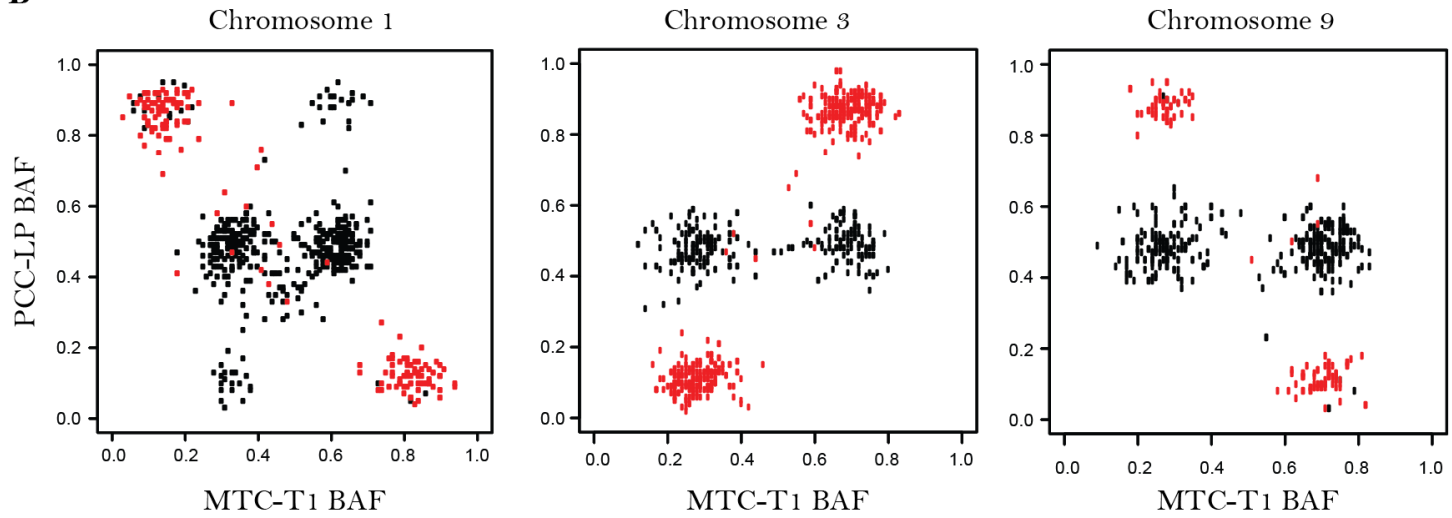

Figure 5.15 CNA common to PCC and T1

(A) High-resolution copy-number analysis of MTC (T1) and PCC (left pole) showing shared copy-number losses (highlighted regions). (B) Allele enrichment analysis of shared regions on chromosomes 1, 3, and 9. Interpret as per Figure 5.10.

function of that gene. If so, it stands to reason that tumour progression within these tissues may follow a common evolutionary pathway with respect to genomic alterations. Comparison of the genomic alterations in the PCC and MTC revealed shared copy-number loss or LOH on chromosome 1p, 3q, and subsections of $9 q$ and $10 q$ (Figure 5.15a). Examination of allele enrichment demonstrated that opposing copies of chromosome $1 \mathrm{p}$ and $9 \mathrm{q}$, and matching copies of chromosome $3 \mathrm{q}$ has been lost (Figure 5.15b), further supporting the idea of compound haploinsufficiency over enrichment of an occult germline mutation. There were no subtle mutations shared between the two disease sites. These results further 
highlight the critical role for alterations to chromosome $1 \mathrm{p}$ and $3 \mathrm{q}$ to the progression of $R E T$-driven tumours.

\subsection{Discussion}

Multiregional sampling within two patients with multifocal disease revealed a diverse array of evolutionary processes, with examples of intertumoural and intratumoural heterogeneity seen in both cases studied. Examination of primary one in case one revealed an extremely diverse genomic landscape with distal regions of the tumour showing as little similarity to each other as to the other synchronous primaries. Furthermore, similar to what has been seen in $V H L$-driven RCC (Fisher et al., 2014), synchronous primaries from the same patient were as diverse as if they had come from separate patients. Despite this, several examples of convergent evolution were seen in both cases, all SDHB-driven tumours demonstrated loss of the wild-type allele through loss of chromosome 1p, similarly, all RET-driven tumour also demonstrated loss of chromosome 1p, albeit for a hitherto unknown reason. Partial or complete loss of chromosome 3 was seen at varying degrees of clonality in PPGL arising in both cases, as well as T1 in case two. The subclonal nature of chromosome 3 loss in several of the PPGLs, as well as the observation that clones $\mathrm{TM}_{\mathrm{P}}$ and $\mathrm{T} 1 \mathrm{Ki} 67$ had lost opposing copies, suggest that chromosome 3 loss is a later event in the progression of both PPGL and MTC. Another example of convergent evolution was seen in the loss of chromosome $2 \mathrm{q}$ in primaries one and four, a genomic alteration that seems to imbue significant growth advantage. The rarity of chromosome $2 q$ losses suggests that this event, in contrast to commonly occurring chromosome 3 events, may be under a positive selective pressure specific to the genome of this patient. This finding suggests that in some cases the presence of secondary driver mutations within the germline may influence the evolutionary pathway taken by tumours. There has been some discussion surrounding the possibility that secondary genomic hits may occur early in embryonic development (Eisenhofer et al., 2011). Daughter cells harbouring the genetic lesion then migrate to disparate locations within the body giving rise to synchronous or metachronous primaries. Without knowledge of their common ancestry, these tumours would appear to exhibit convergent evolution. However, this is unlikely to explain the convergence seen in the cases presented here. For instance, the loss of chromosome 2q, present in both primaries 1 and 4 of P018 is not ubiquitous in primary 1, suggesting that the event has occurred later in tumour evolution. Examples of both linear and branched evolution were seen in both cases and both tumour types. Several of the tumours appeared to have undergone periods of both linear evolution, whereby selective sweeps resulted in a single dominant clone, and branched evolution, which results in several coexisting subclones. In instances of branched evolution, it is unclear from the data whether maintenance of clonal diversity is the result of mutually beneficial cooperation or simply the failure of any one clone to outcompete others. There were no specific examples 
of convergent evolution seen with regard to subtle mutations in either case. Coupled with the low mutation rate and the observation that several early mutation events were non-deleterious, this may suggest that subtle mutations are of secondary importance in the early development of both PPGL and MTC. However, the detection of an early MAX mutation in MTC sample T1 is of particular interest given the mounting evidence for the importance of $M A X$ mutation in neuroendocrine cancer.

Primary three from case study one demonstrated maintenance of clonal diversity, where rather than a single clone becoming dominant, two or more clones were present in equal proportions across the tumour regions sampled. This is of particular interest in considering the potential for mutually beneficial relationships between diverse subclones. A 2010 study of glioblastoma multiforme demonstrated that cells possessing over-activation of EGFR are able to confer a growth advantage to surrounding EGFR wild-type cells through cytokine signalling (Inda et al., 2010). Similarly, a recent study demonstrated clonal cooperation in WNT-driven mouse mammary cancers, whereby Hras-mutant basal-type tumour cells were dependent on Hras-wild-type WNT-producing luminal-type tumour cells (Cleary et al., 2014). Interestingly, depriving the basal-type cells of an exogenous WNT source often lead to development of self-sufficient basal-type clones through mutation of $\beta$-Catenin. Furthermore, clonal cooperation has also been implicated in metastatic spread. A recent study of metastatic prostate cancer found that multiple distinct clones present at the metastatic site could be detected as pre-existing within the primary tumour suggesting polyclonal seeding (Gundem et al., 2015). The study was unable to discern whether this represents an initial collective migration or a single clone providing a beachhead by shaping a permissive microenvironment for the arrival of other clones. Either way, clonal cooperation likely plays a role in the metastasis of prostate cancer. What role, if any, cooperation between clones plays in PPGL development remains to be established.

Interestingly, despite both being driven by an initial RET mutation, the MTC and PCC from case two exhibit quite distinct genomic landscapes. The MTC exhibits considerably more genomic instability on both the level of large copy-number alterations and subtle mutations. The PCC, as is typical of these tumours, exhibits predominantly copy-number loss events, whereas the MTCs demonstrate an equal tendency towards loss and gain events. These observations are particularly interesting to note given the clinical differences between PCC, a mostly benign and indolent tumour, and MTC, an aggressive and often metastatic disease. These data do not provide sufficient evidence to determine whether the increased genomic instability is a consequence or cause of the more rapid growth seen in MTC. There is also the possibility that parafollicular cells, the cell of origin for MTC, are inherently more tolerant of genomic instability therefore undergo less clonal culling due to harmful alterations. 
Traditionally, molecular diagnostic assays are performed using surgically resected tissue or tissue specimens obtained through methods such as fine needle aspiration or core needle biopsy. However, in cases where one or more disease sites may not be accessible, or patients have contraindications, such as undergoing treatment with antiangiogenic drugs (Hompes \& Ruers, 2011), these procedures may not be feasible. Furthermore, even in the absence of contraindications, a biopsy procedure may have adverse effects on a patient's health, with a small study reporting biopsy related complications in 14 of 20 (70\%) patients (Vanderveen et al., 2009). Increasing recognition of intertumoural and intratumoural heterogeneity within a single patient has also cast doubt on the utility of single point biopsy (Crowley et al., 2013). As cfDNA is safely and easily accessible, and is thought to more robustly represent tumour diversity, "liquid-biopsy"-based assays have been proposed as a viable method for overcoming some of the limitations of traditional biopsy. These methods allow non-invasive detection of potential markers for treatment response, such as $B R A F$ mutations for vemurafenib in melanoma (Gonzalez et al., 2013) or $K R A S$ mutations for cetuximab or panitumumab in colorectal cancer (Allegra et al., 2009), as well as monitoring of potential markers of treatment resistance (Murtaza et al., 2013). Cell-free DNA can also provide a safe and cost-effective adjunct to disease surveillance, whereby the levels of a tumour-specific mutation are monitored to detect residual or recurrent disease (Diehl et al., 2008). The success of cfDNAbased assays is contingent on their ability to sensitively detect tumour-specific biomarkers from within a mixed population of normal and tumour-derived cfDNA. The degree of sensitivity required is dependent on the application, for example, an assay to detect a targetable mutation present in the bulk of a tumour would require lower sensitivity than one to detect a marker of resistance in a minority of tumour cells. The data presented here suggest that exome sequencing of cfDNA would be sufficiently sensitive to detect disease-wide targetable mutations but, importantly, it would not be sufficient for low abundance markers of resistance. While exome sequencing was used for discovery in the context of this chapter, the extent of the regions covered makes exome sequencing impractical for a diagnostic setting as it both reduces sensitivity and increases cost. A more appropriate approach in a clinical context would be to use a targeted panel, allowing for ultra-deep sequencing which has the potential to detect less abundant mutations. Given the apparent importance of copy-number alterations to the development of both MTC and PPGL, it is likely that any clinically relevant diagnostic assay based on cfDNA would need to be sensitive to these changes, a challenge that is currently being addressed (Xu et al., 2015). However, any approach such as this is contingent on the development of appropriate biomarkers.

This chapter utilised the unique resource of multiple synchronous primaries to explore the nuances of tumour evolution on a uniform genetic background. And in doing so, revealed a startling array of variation, highlighting again the significant challenge that tumour heterogeneity presents to modern 
oncology. However, several instances of convergent evolution provide clues to obligatory genomic events in the development of MTC and PPGL, further research into these critical regions may reveal targets for potential therapeutics. 


\section{Concluding Remarks and Future Directions}

\subsection{The Emerging Genomic Landscape of PCC}

The previous three decades have seen an incredible expansion in the understanding of the biology of PPGL. Beginning with the discovery that mutations in the RET and $V H L$ genes give rise to MEN2 and von Hippel-Lindau syndromes respectively, there has been a steady increase in the number of both heritable and somatically altered PCC driver genes. These discoveries have been due in part to the rapid expansion of genomics technologies, including improvements in dideoxynucleotide sequencing, the advent of microarray based assays for gene-expression and cytogenetics, and, most recently, massively parallel sequencing. The advent of whole-transcriptome microarray-based expression profiling technologies facilitated early work that demonstrated PPGL can be clustered into two major groups and ultimately into as many as six minor groups. This striking facet of PCC biology was exploited in order to discover $M A X$ mutant tumours and provide supporting evidence for mutations in $F H$ and $M D H 2$. Despite the expanding repertoire of primary driver genes, there remained a paucity of knowledge surrounding the general genomic landscape of PPGL outside of large-scale copy-number alterations. The aim of this thesis was to utilise cutting edge high-throughput genomics technologies to further refine the genomic alterations that define PPGL. Unsurprisingly, over the same time course several other research groups, including large cancer genomics consortium The Cancer Genome Atlas (TCGA), have attempted to do the same. While the TCGA data have not been formally published at the time of writing, the data is available in the public domain. In combination, the published studies and TCGA data represent exome sequencing for over 250 cases, with many also having undergone high-resolution copy-number, transcriptome, miRNA, and methylation analysis. This incredible wealth of data has begun to provide valuable insight into the underlying genomic architecture of this highly heterogeneous disease.

In the work presented in this thesis, genomic profiling of 40 PPGL revealed a highly stable genome defined by a low mutational load and large segmental copy-number alterations. With very few exceptions, copy-number alterations were seen as chromosomal loss events, suggesting that PCC is driven more by failure of tumour suppressor genes than activation of oncogenes. The mutational spectrum of tumours has been used to uncover mutational processes in a variety of tumour types, similar analysis in PCC revealed a predominance of $\mathrm{C}>\mathrm{T}$ transitions, a signature thought to be associated with ageing. Concordant with the low mutational burden, none of the known mutagenic signatures, such as those associated with carcinogen exposure or the APOBEC family of cytidine deaminases, were present. Despite the low mutation frequency, combining the data presented in Chapter Three with publically available TCGA and CastroVega et al. data sets reveals deleterious alterations in over 5300 genes (pre-publication provisional TCGA 
used). Of these, a startling 69\% (3657/5308) were mutated in only a single sample, while only $14 \%$ (757/5308) of genes were seen to be mutated in three or more samples. Estimates of discovery power by Lawrence et al. (2014) suggest that, based on the rate of 0.3 mutations per megabase proposed Castrovega et al. for PPGL, to detect genes mutated in 1,2,3,5, and $10 \%$ of cases would require sequencing of approximately $1500,500,300,160$, and 70 cases, respectively. This suggests that with the current pool of published data, there is only sufficient power to detect genes mutated in greater than around $4 \%$ of cases, or in the case of this specific study, greater than $10-15 \%$ of cases.

Attempts to stratify the genes in this study using pathway analysis tools met with limited success. While it is possible this results from an under-representation of neuroendocrine-specific biology in the pathway databases utilised, it may also be that the study was under-powered to detect a sufficient number of infrequently mutated pathway members. Beyond the established cancer genes, manual integration revealed several broad functional classes including genome maintenance, epigenetic control, and cytoskeletal remodelling. The importance of epigenetic control has since been underscored by subsequent studies showing that $A T R X$ (Fishbein et al., 2015) and KMT2D (Juhlin et al., 2015) are somatically mutated in $12.6 \%$ and $11 \%$ of PCC, respectively. Although, one might note that KMT2D was not found to be somatically mutated in the Castro-Vega et al. data set, and only appeared in a single sample from this study $(1 / 40,2.5 \%)$ and the TCGA data set $(1 / 179,<1 \%)$, suggesting that considerable work remains to establish the true frequency of recurrently mutated genes.

Interpreting the extensive list of non-recurrent mutations presents a significant challenge. On the one hand, the diversity of mutations may reflect the heterogeneous nature of PCC, mirroring the distinct array of pathways affected across the various subtypes. On the other, it may represent something of a red herring, with the majority simply being passenger events that play no part in tumourigenesis. In this scenario, PCC development may rely less on subtle mutations and more on alternate mechanisms of gene disruption such as chromosome loss, functional and non-functional fusion genes, or dysregulation of epigenetic modifiers and miRNAs (Castro-Vega, Letouze, et al., 2015). At present, interpretation of gene mutations is largely dependent on annotation derived from various experimental sources, the reality may be that determining the importance of infrequently mutated genes is beyond the scope of the current knowledge base. As such, the data will need to be periodically revisited within the evolving framework of cellular biology. 


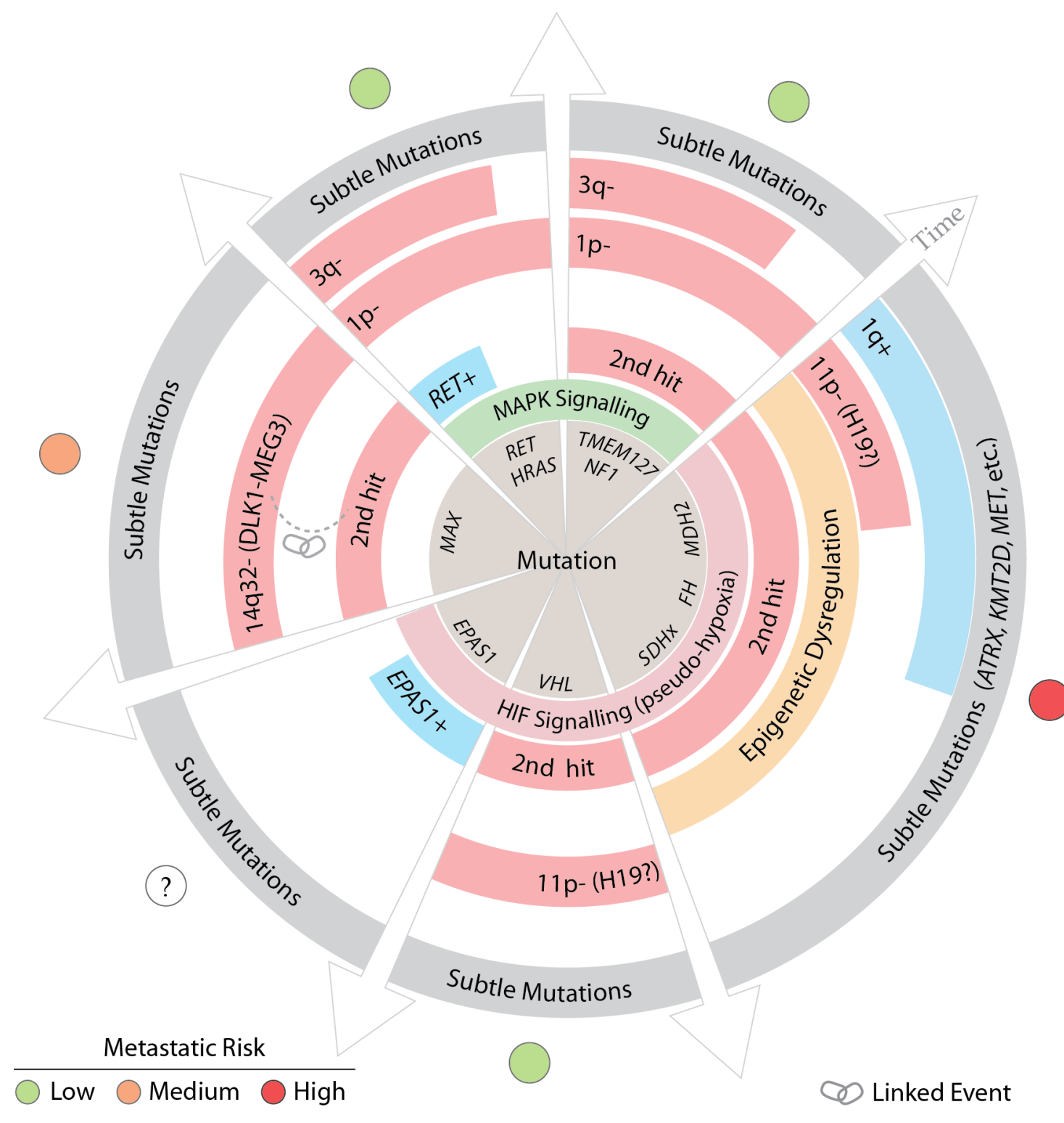

Figure 6.1 Recurrent evolutionary paths in PPGL

Genomic profiling has revealed recurrent patterns of copy-number alterations contingent on the underlying driver mutation. Some of these chromosomal alterations appear to occur exclusively as early events (e.g. loss of chromosome $11 \mathrm{p}$ in VHL type tumours) or at variable time points (e.g. chromosome 3 loss in MAPK type tumours). Chromosome loss events are drawn in pink and gain events in blue, the length of each bar relative to the containing segment indicates the proportion of tumours in which the alteration is seen. 
Through the work presented in Chapter Three and additional publications (Castro-Vega, Letouze, et al., 2015; Crona et al., 2015), it is clear that certain patterns of copy-number alteration in PCC are strongly correlated with the underlying driver mutation and, by extension, expression cluster. The most commonly seen events are those that result in loss of the wild-type allele corresponding to the driver mutation. Beyond this, loss of chromosome $1 \mathrm{p}$ and $3 \mathrm{q}$ are seen in greater than $80 \%$ of RTK/Cluster $2 \mathrm{~A}$ type tumours, while tumours driven by $V H L$ exhibit almost ubiquitous loss of chromosome $11 \mathrm{p}$ as well as frequent loss of 3q. These patterns suggest that each driver requires a distinct cascade of genomic alterations for tumourigenesis to occur. The extent and uniformity of the altered regions indicates that multiple gene loci across the affected chromosomes are likely to be involved. Despite the tantalising window into PCC biology that these alterations offer, surprisingly few candidate loci have been proposed. Tumours driven by both loss of function mutations in $V H L$ or $S D H D$ have been shown to exclusively lose the maternal copy of chromosome $11 \mathrm{p}$, leading investigators to suspect the maternally imprinted $H 19$, a long non-coding RNA which functions as a tumour suppressor (Margetts et al., 2005; Yeap et al., 2011). Similarly, $M A X$ mutations exhibit a parent-of-origin effect whereby only inheritance on the paternal copy and subsequent loss of the maternal copy permits tumourigenesis. Combined miRNA and CNA profiling has recently implicated the maternally imprinted DLK1-MEG3 miRNA cluster located on chromosome 14 (Castro-Vega, Letouze, et al., 2015). As no such allelic bias has been observed for the remaining regions, which are frequently altered in PCC, and traditional methods of finding the smallest region shared between tumours are hampered by uniformly large segmental events, identifying the critical gene loci affected by CNA remains a significant challenge in understanding PCC biology.

\subsection{Subtypes and the Search for Novel Drivers}

Consensus clustering of a compendium of publically available PPGL expression data revealed six robust subtypes correlating closely with those previously published (Burnichon et al., 2011). Tumours harbouring SDHx and $V H L$ mutations were represented by their own respective subclasses, and the remaining MAPK/mTOR-type tumours held membership across three subclasses (RTK 1-3). A sixth subclass, largely devoid of cases with a known driver in the compendium data set, was revealed through cross-platform classification of a secondary data set to contain MAX mutant tumours and termed MAXlike.

Cross-platform classification of RNA-seq gene-expression data was able to accurately classify $93 \%$ of samples for which a mutation could be detected by exome sequencing. Samples lacking a detectable mutation were not evenly dispersed among the subgroups. Of the nine samples without a detectable 
mutation, a single sample classified into each of the RTK 1, 2, 3 and VHL classes, while the remaining five samples classified into the MAX-like class. In total, a driver mutation was detected in $75.5 \%(31 / 40)$ of samples, a figure that matches closely with current estimates that $70 \%$ of PCC can be explained by either a germline or somatic mutation in one of the known genes (Castro-Vega, Lepoutre-Lussey, et al., 2015). The high percentage of cases that can now be solved makes the absence of any detectable driver in the MAX-like group all the more striking. As mentioned above, the name MAX-like was initially assigned after observing that $M A X$-mutant tumours in an independent published microarray set classified into this class. Subsequently, further support for the membership of MAX-mutant PCC in the MAX-like (which equates to Cluster 2B) group was provided through application of the Nanostring classifier assay to three known $M A X$ cases. Despite this, membership of $M A X$ tumours in the MAX-like/Cluster 2B class remains controversial in the literature, with a recent review in the journal Oncogene asserting that MAXmutant tumours belong in Cluster 2A (RTK 1/3) (Castro-Vega, Lepoutre-Lussey, et al., 2015). As the $M A X$-driven tumours utilised in the Nanostring assay were sourced from related individuals, and therefore harboured identical mutations, the possibility that subtype membership of $M A X$-mutant tumours is variable depending on the exact genetic alteration cannot be excluded. Additional profiling and classification of a diverse set of $M A X$-mutant tumours will be required to provide clarification.

As the MAX-like class accounts for approximately $10 \%$ of PPGL and mutations in MAX are estimated to only contribute to around 1\% of PPGL (Burnichon, Cascon, et al., 2012), the MAX-like class would appear to be fertile ground for gene driver discovery. However, novel drivers within this class have proven elusive. Between the work presented in Chapter Three and that of Castro-Vega, Letouze, et al. (2015), exome sequencing has been performed on sixteen MAX-like/Cluster-2B tumours. Despite this reasonably extensive discovery set no candidate genes have been published. Across both data sets, only a single gene was mutated in more than one sample, OR13C5, a member of the olfactory receptor family, which was found not to be expressed in any of the PPGL profiled in Chapter Three. This raises two possibilities, either the MAX-like group is composed of a highly heterogeneous group of low frequency driver mutations precluding recurrence in a small sample set, or some or all of the remaining driver mechanisms lie beyond the scope of exome sequencing, potentially involving mechanisms such as gene fusions, promoter/enhancer mutations, epigenetic gene silencing, or dysregulation of lncRNA or miRNA.

As previously mentioned, abolition of a cluster of maternally imprinted miRNA, known as the DLK1$M E G 3$ cluster, has been implicated in the parent-of-origin effect seen in $M A X$-mutant tumours. More recently Castro-Vega et al. demonstrated that downregulation of this cluster of miRNAs was also present in many Cluster 2B tumours lacking a $M A X$ mutation. The authors speculated that loss of heterozygosity 
in the region of $14 \mathrm{q} 32$ and the resulting loss of $D L K 1-M E G 3$ expression may be driving tumourigenesis in these cases. However, DLK1-MEG3 down regulation was not exclusive to Cluster 2B tumours being seen also in tumours driven by VHL, NF1, RET, and TMEM127. As such, it remains unclear whether loss of the DLK1-MEG3 miRNA cluster is acting as a primary or secondary driver in MAX-like/Cluster 2B tumours. Strikingly, besides alterations to chromosome 11, MAX-like tumours do not display the copynumber changes typical of the other subtypes, with very few tumours displaying loss of chromosomes $1 \mathrm{p}$, 3 , or 17. Furthermore, loss of chromosome 4 and gain of chromosomes 7 and 8 were seen in several MAX-like/Cluster 2B samples. In combination, expression profiling and CNA analysis suggest that MAX-like tumours utilise an oncogenic pathway distinct from that of the other subtypes. Identifying the primary drivers in these cases will likely require an integrated genomics approach examining genetic and epigenetic changes across the whole genome.

\subsection{Classification as a Diagnostic and Research Tool}

The expense of traditional single-gene diagnostic tests has led to the development of decision-tree algorithms for prioritising genes (Benn et al., 2006; Jafri et al., 2012). Currently clinical factors such as age of onset, disease location, and catecholamine profile are utilised for gene selection. Gene-expression based diagnostic classification can provide a cost-effective and highly informative augmentation to existing decision algorithms, helping to alleviate the time and cost burden associated with genetic testing.

Increasingly, as diagnostic testing shifts from single gene tests to high-throughput approaches, the need for gene prioritisation will diminish. However, these methods have distinct technical limitations including variants missed owing to poor sequence coverage and false positives caused by misalignment to the reference genome (Meldrum et al., 2011). In this scenario, classification can be used for quality assurance purposes, mutations detected that do not conform to the assigned class can be put through more rigorous assessment, conversely where a mutation is not found in the genes indicated by classification, further assessment of the sequencing data for those genes may be initiated. A significant challenge facing diagnostic gene testing is that of unclassified variants, also referred to as variants of unknown significance. The increasing importance of this issue was exemplified by recent publications describing previously unreported VHL mutations in patients with ambiguous family history (Russell et al., 2015; Sexton et al., 2015). Classification in cases such as these can provide valuable guidance as to the participation of a variant of unknown significance. In cases where the driver mutation cannot be ascertained, classification may still be used to guide clinical management. For instance, if a tumour were to classify into a subtype with high metastatic risk, a more stringent surveillance regime may be indicated. Classification in these cases may also help guide selection of targeted therapies, such as the use of 
tyrosine kinase inhibitors with anti-angiogenic properties such as sunitinib or pazotanib, which may be more efficacious on pseudohypoxia type tumours (Hata et al., 2014; Joshua et al., 2009).

Chapter 4 described the development and validation of a new diagnostic test for classification of PPGL tumours based on the underlying genetic driver of the disease. Initially a six-class classification was attempted based on the subtypes detected through consensus clustering. However, testing in a cohort of 38 clinical samples revealed that the RTK2 and RTK3 subtypes were likely associated with the presence of non-neoplastic cells. Five- and four-class models of classification were proposed to ameliorate factors such as tumour purity and heterogeneity, both achieving equally high classification accuracy of $92.31 \%$ for samples with a known driver. By reducing the classifier to represent the three major biological classes (pseudo-Hypoxia, MAPK/RTK signalling, and MAX-like) a classification accuracy of $100 \%$ was achieved. For the purposes of diagnostic testing a four class classifier is likely optimal, representing a trade-off between driver specificity and classification accuracy. Moving forward, a prospective multi-site evaluation of the assay will be required to gauge clinical utility, reproducibility, and accuracy.

The fact remains, however, that the biology driving RTK2 and RTK3 tumours requires elucidation. It would appear in some cases assignment to the RTK2 class simply indicates contamination with surrounding cortical tissue which can be eliminated with careful sample preparation. While in others, macrodissection to the tumour margin failed to eradicate the expression of cortical genes, potentially indicating an admixture of cortical and chromaffin type cells at the tumour periphery. In which case, ascertaining the relationship between these distinct cell types will be integral to understanding the biology of this subtype. The RTK3 subtype, which has not been described in previously published subtype analyses, is characterised by overexpression of the same genes as RTK1 tumours (e.g. PNMT and RET), consistent with the notion of a well-differentiated disease. However, several genes that distinguish RTK3 from RTK1 are indicative of inflammatory processes ( $R R G N, C S F 1 R, T Y R O B P)$ and wound repair (CCL2, CYR61, CTGF). In regard to expression of these genes, RTK3 is more closely aligned with the pseudo-hypoxia type tumours perhaps indicating a more vascularised tumour. Correlation of clinical and histological features with subtype across a larger cohort of tumours will be required to ascertain the clinical importance of the RTK2 and RTK3 subtypes.

Moving forward, it will be important to assess how the classifier performs with low prevalence drivers. Recently described drivers such as $M D H 2, F H$, and particularly EPAS1, may have gene-expression profiles that distinguish them from the other pseudo-hypoxia type tumours. If so, the signature may have been obfuscated in the current analysis by underrepresentation in the discovery data sets. Application of 
the Nanostring assay to a sufficient number of cases with these genotypes may facilitate retraining of the assay to allow accurate identification. If the current gene set proves inadequate for the task, differential expression analysis of whole transcriptome studies with a sufficient number of representative samples could be used to derive a gene set to augment the assay.

In addition to use in clinical diagnostics, the classification assay is also a valuable research tool. The utilisation of subtyping to interpret potential novel drivers has already proven useful in PCC. Previously, hierarchical clustering was used to demonstrate shared biology between $S H D x$ tumours and the proposed novel drivers $M D H 2$ and $F H$. Similarly, PCC tumours with $M A X$ mutations were revealed through exome sequencing of tightly clustering tumours without a known mutation. In addition to providing a framework for interpreting VUS in known PCC genes, a classification assay can also be used to guide the search for drivers in unexplained cases. For instance, if a tumour without a detectable driver were to classify as an SDHx type tumour, investigating novel mechanisms of gene inactivation or examining additional members of the TCA cycle may prove fruitful. This is particularly important for the $M A X$-like group which, as previously discussed, represents something of a last frontier in the discovery of PPGL driver genes. The ability to identify these tumours for further study in a rapid and cost effective manner is of the utmost importance.

\subsection{Cousins not Twins: Lessons from Sub-Clones and Synchronous Primaries}

Detailed genomic profiling of two patients exhibiting synchronous primaries in the context of a germline mutation revealed considerable intertumoural and intratumoural heterogeneity. Among four synchronous paragangliomas profiled, there were no commonly mutated genes, nor were there instances of mutations within the same gene arising independently within the same tumour, a phenomenon seen in other tumour types (Gerlinger et al., 2012). In contrast, subclonal loss of chromosome 3 was seen in three of four tumours, and loss of chromosome $2 \mathrm{q}$ was seen in two of four tumours, suggesting that these events are under strong positive selection but not a pre-requisite for tumourigenesis. The observation that identical copies of chromosome $2 \mathrm{q}$ were lost in both tumours, combined with the rarity of alterations to chromosome $2 \mathrm{q}$ in $S H D B$ tumours, suggests that the loss may be enriching a secondary driver or modifier gene variant present in the germline. This implies that while some aspects of PPGL evolution, such as loss of chromosome 3q, are independent of the patient genome, others may stem from the tumour leveraging an existing genomic vulnerability. As more genomic data become available, cataloguing these less common chromosomal alterations and correlating them with germline variants may provide crucial insight into secondary drivers in PPGL. 
Multiregional sampling of a single tumour (Pr1) demonstrated extensive heterogeneity resulting from subclonal divergence. Comparison of diametric regions of the tumour revealed only a single somatic mutation and two copy-number alterations, one being the obligatory loss of chromosome $1 \mathrm{p}$, as shared. This degree of clonal diversity, however, is likely an extreme example as multiregion sampling of a $R E T$ mutant tumour and a second $S D H B$ mutant tumour revealed multiple shared mutations and copy-number alterations. However, all tumours displayed one or more subclonal copy-number alterations, suggesting that PPGL are routinely genomically heterogeneous. This is consistent with the findings of Crona et al. (2015) who demonstrated, in what is currently the largest study examining PCC heterogeneity, discrepant CNA patterns through multiregion sampling in 18 of 21 cases. Although intratumoural heterogeneity may influence clinical factors such as imaging tracer uptake, in cases of benign PPGL surgical resection renders the issue of heterogeneity largely academic. However, the concept is likely crucial to understanding, diagnosing, and treating tumours with malignant potential.

\subsection{Tumour Heterogeneity and Malignant Potential}

The estimated five year survival for benign PCC is around 95\% (Goldstein et al., 1999). As the majority of morbidity and mortality associated with benign disease results from the effects of excess catecholamine secretion, it is unlikely that understanding the genomic landscape of PPGL is going to improve this figure. It is, however, important to first understand the genomic variability present in benign disease in order to begin to distinguish what differentiates benign from malignant disease. In contrast to benign disease, five year survival rates for malignant disease has been reported to be between 34 and $60 \%$ (Parenti et al., 2012). Furthermore, metastatic recurrence has been shown to occur as much as 15 years after initial diagnosis (Goldstein et al., 1999) and as discussed previously, there are currently no official guidelines from the WHO for distinguishing benign from malignant disease. Numerous biomarkers have been correlated with metastatic behaviour (Fernandez et al., 2013; Liu et al., 2014; Meyer-Rochow et al., 2010; Patterson et al., 2012; Qi et al., 2012; Sandgren, Andersson, et al., 2010; Suh et al., 2009; Xu et al., 2013), however, none have offered significant predictive value. Despite this, there is now a general consensus that large tumour size, extra-adrenal location, and the presence of an SDHB mutation are adverse prognostic factors. Drawing on the observations made in Chapter Five, it seems logical that tumour size may in fact be acting as a surrogate measure of tumour heterogeneity. The data presented for the evolution of MTC demonstrated sequential acquisition of genomic alterations presumably culminating in a clone with metastatic potential. A similar model in PPGL is supported by findings that metastatic PPGL exhibit a greater degree of genomic heterogeneity than their benign counterparts (Crona et al., 2015). This, however, begs the question that if metastatic potential is a matter of procuring the correct 
genomic alterations, why then do $S D H B$ mutant tumours have a higher propensity for metastatic behaviour than MAPK/RTK type tumours? While it seems likely that the answer to this will lie, at least in part, with the epigenetic dysregulation caused by oncometabolite accumulation (Letouze et al., 2013; Xiao et al., 2012), given that only a portion of $S D H B$ mutant tumours become malignant, it is unlikely that the presence of these epigenetic changes are sufficient to drive metastasis without acquisition of further genomic alterations.

Strikingly, the majority of studies comparing benign and malignant disease have opted to treat each group as homogeneous, simply comparing benign tumours with malignant without consideration for the underlying driver biology. Given that each PCC subtype appears to follow unique oncogenic processes, it stands to reason the process of metastatic transformation may be equally distinct. As such, it seems prudent to first stratify benign and malignant samples by subtype prior to comparison.

Historically, comparison has also been performed as malignant primary versus benign primary. As was seen in Prl of case study one in Chapter Five, significant regional differences can exist within a single tumour, therefore in selecting a portion of a malignant primary for study, one could potentially fail to obtain any cells with metastatic potential. Moving forward, by utilising increasingly high-sensitivity genomics techniques, studies similar to that performed for T1 and T1M in Chapter Five, whereby the metastatic clone is identified within the primary tumour, can provide a powerful research model. This approach facilitates comparison of the parental metastatic clone with coexisting clones, metastatic deposits, and unrelated benign and metastatic tumours, allowing identification of specific sub-clonal alterations that imbue cells with the ability to metastasise. This, of course, subscribes to the paradigm that the genomic alterations which facilitate metastasis occur late in the evolution of a tumour. This view has been questioned by those suggesting that, firstly, metastatic seeding has a high rate of failure and, secondly, the alterations that provide metastatic capability provide no growth advantage (Bernards \& Weinberg, 2002). In this scenario the genomic alterations that provide metastatic capability would need to occur earlier in tumour development to be present in a sufficient number of cells for a successful seeding event to occur. Ideally, future experimental design will provide consideration for both models.

In addition to somatic changes, metastatic potential may be affected by the presence of polymorphisms in modifier genes as has been seen for BRCA1/2-related breast and ovarian cancer penetrance (Barnes \& Antoniou, 2012; Peterlongo et al., 2015). Identification of such loci is complex, perhaps requiring identification of unrelated families harbouring an identical PPGL driver mutation but exhibiting contrasting rates of metastatic disease. In such a scenario, genomic profiling of tumours from multiple 
probands may reveal uncommon but recurrent genomic alterations such as that seen on chromosome $2 \mathrm{q}$ in Chapter Five.

As discussed previously, patients can be stratified into high and low metastatic risk groups based on the causative gene mutations, with $S D H B$-mutant tumours presenting the highest risk. There is some evidence that this risk can be further stratified based on the exact mutation present. Ricketts et al. (2010) performed a genotype to phenotype analysis of 295 patients with $S D H B$-associated PPGL from 125 kindreds. This study demonstrated a higher, albeit not statistically significant, propensity for metastasis in patients with truncating or missense mutations in the first 300 nucleotides of the transcript. The major challenge to performing analyses such as this is acquiring sufficient samples with a given genotype. Further clarification will likely come from either meta-analysis of existing data, or a large multi-site effort.

Deciphering the relative contribution of modifier genes, epigenetic reprogramming, genotype-phenotype relationships, as well as early and late somatic alterations will likely be a considerable focus for the field of PPGL biology in the near future.

\subsection{Conclusion}

The work presented in this thesis represents a first foray into understanding the somatic changes driving PPGL development, providing preliminary insight into the biological and evolutionary processes underlying tumour development. PPGL has continually displayed remarkable heterogeneity both on the intertumoural level, demonstrating diversity of transcriptome profiles, chromosomal alterations, and somatically mutated genes, and the intratumoral level, with evidence of divergent clones existing in spatially distinct regions. Moving forward, understanding how the various layers of disease heterogeneity contribute to disease progression will be of the utmost importance in ensuring the best outcome for patients presenting with PPGL.

Furthermore, this thesis described the development of a robust assay for subtyping PPGL using geneexpression data. In a clinical setting, the assay could be a valuable adjunct to genetic testing by providing orthogonal validation of a genetic testing result, as well as providing guidance in interpreting variants of unknown significance, and potentially in making clinical management decisions in the absence of a detectable mutation. This direct translational outcome supports the rationale for genomic studies even for rare tumours. 


\section{Appendices}

\section{Appendix A PyClone Configuration File}

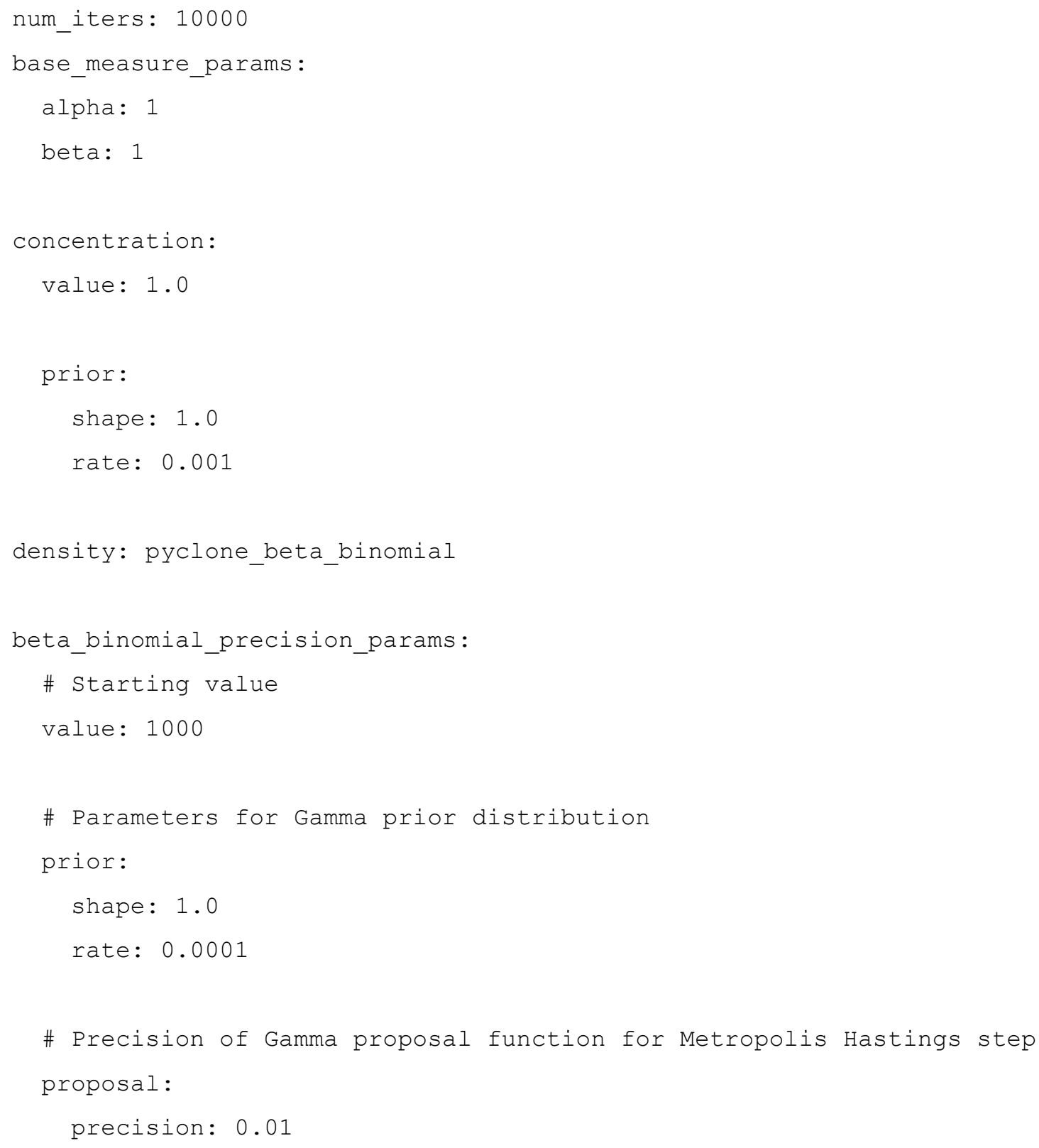


Appendix B Nanostring Validation Cohort

\begin{tabular}{|c|c|c|c|c|c|c|c|c|c|c|}
\hline Sample ID & PCC/PGL & Sample & Dx Age & Location & Source & Genotype & Six Class & Five Class & Four Class & Three Class \\
\hline RBH01-R1 & PCC & FFPE & 14 & Bilateral & $\mathrm{RBH}$ & $M A X$ & MAX-Like(H) & MAX-Like(H) & MAX-Like(H) & MAX-Like(H) \\
\hline RBH01-R2 & PCC & FFPE & 14 & Bilateral & $\mathrm{RBH}$ & MAX & MAX-Like(M) & MAX-Like(L) & MAX-Like(M) & MAX-Like(M) \\
\hline RBH02 & PCC & FFPE & 50 & Right adrenal & $\mathrm{RBH}$ & MAX & MAX-Like(H) & MAX-Like(H) & MAX-Like(H) & MAX-Like(H) \\
\hline $\mathrm{RBH03}$ & PCC & FFPE & 42 & Bilateral & $\mathrm{RBH}$ & MAX & MAX-Like(H) & MAX-Like(H) & MAX-Like(H) & MAX-Like(H) \\
\hline RNSH01 & PCC & FFPE & 35 & Right adrenal & Kolling & NF1 & RTK1(H) & RTK1(H) & $\operatorname{RTK}(\mathrm{H})$ & $\mathrm{RTK}(\mathrm{H})$ \\
\hline RNSHO2 & PCC & FFPE & 45 & T3/T4 Spine & Kolling & $S D H B$ & $\mathrm{SDHx}(\mathrm{M})$ & $\mathrm{SDHx}(\mathrm{M})$ & $\mathrm{SDHx}(\mathrm{H})$ & pHyp(H) \\
\hline RNSHO3 & PCC & FFPE & 41 & Omentum & Kolling & $S D H B$ & $\mathrm{SDHx}(\mathrm{H})$ & $\mathrm{SDH} x(\mathrm{H})$ & $\mathrm{SDH} x(\mathrm{H})$ & $\mathrm{pHyp}(\mathrm{H})$ \\
\hline RNSHO4 & PGL & FFPE & 40 & Retroperitoneal & Kolling & $S D H B$ & $\mathrm{SDHx}(\mathrm{H})$ & $\mathrm{SDHx}(\mathrm{H})$ & $\mathrm{SDHx}(\mathrm{H})$ & $\mathrm{pHyp}(\mathrm{H})$ \\
\hline RNSH05 & PCC & FFPE & 31 & Left adrenal & Kolling & HRAS & RTK3(H) & RTK3(M) & $\mathrm{RTK}(\mathrm{H})$ & $\mathrm{RTK}(\mathrm{H})$ \\
\hline RNSHO6 & PCC & FFPE & 34 & Bilateral adrenal & Kolling & $V H L$ & $\mathrm{VHL}(\mathrm{H})$ & $\mathrm{VHL}(\mathrm{H})$ & VHL(H) & $\mathrm{pHyp}(\mathrm{H})$ \\
\hline RNSH07 & PGL & FFPE & 35 & Retroperitoneal & Kolling & $S D H B$ & $\mathrm{SDHx}(\mathrm{M})$ & $\mathrm{SDH} x(\mathrm{H})$ & $\mathrm{SDH} x(\mathrm{H})$ & $\mathrm{pHyp}(\mathrm{H})$ \\
\hline RNSH08 & PCC & FFPE & 31 & Right adrenal & Kolling & $V H L$ & VHL(M) & VHL(M) & VHL(M) & $\mathrm{pHyp}(\mathrm{H})$ \\
\hline RNSH09 & PCC & FFPE & 39 & Right adrenal & Kolling & $V H L$ & $\mathrm{VHL}(\mathrm{H})$ & $\mathrm{VHL}(\mathrm{H})$ & $\mathrm{VHL}(\mathrm{H})$ & $\mathrm{pHyp}(\mathrm{H})$ \\
\hline RNSH10 & PCC & FFPE & 40 & Left adrenal & Kolling & $V H L$ & $\mathrm{VHL}(\mathrm{L})$ & VHL(M) & $\mathrm{VHL}(\mathrm{H})$ & $\mathrm{pHyp}(\mathrm{H})$ \\
\hline RNSH11 & PCC & FFPE & 24 & Adrenal & Kolling & TMEM127 & RTK1(H) & RTK1(H) & RTK(H) & RTK(H) \\
\hline RNSH12 & PCC & FFPE & 37 & Bilateral adrenal & Kolling & TMEM127 & RTK3(H) & RTK3(H) & RTK(H) & RTK(H) \\
\hline RNSH13 & PCC & FFPE & 31 & Right adrenal & Kolling & $R E T$ & RTK3(H) & RTK3(H) & RTK(H) & RTK(H) \\
\hline RNSH14 & PCC & FFPE & 37 & Left adrenal & Kolling & $R E T$ & RTK1(H) & RTK1(H) & $\mathrm{RTK}(\mathrm{H})$ & RTK(H) \\
\hline RNSH15 & PGL & FFPE & 27 & Left adrenal & Kolling & SDHB IHC Neg & $\mathrm{SDHx}(\mathrm{L})$ & $\mathrm{SDHx}(\mathrm{L})$ & $\mathrm{SDHx}(\mathrm{L})$ & pHyp(M) \\
\hline RNSH16 & PCC & FFPE & 60 & Carotid & Kolling & SDHB IHC Neg & $\mathrm{SDHx}(\mathrm{H})$ & $\mathrm{SDHx}(\mathrm{H})$ & $\mathrm{SDHx}(\mathrm{H})$ & $\mathrm{pHyp}(\mathrm{H})$ \\
\hline RNSH17 & PGL & FFPE & 21 & Retroperitoneal & Kolling & SDHB IHC Neg & $\mathrm{SDHx}(\mathrm{H})$ & $\mathrm{SDHx}(\mathrm{H})$ & $\mathrm{SDHx}(\mathrm{H})$ & $\mathrm{pHyp}(\mathrm{H})$ \\
\hline RNSH18 & PGL & FFPE & 35 & Carotid & Kolling & SDHB IHC Neg & $\mathrm{SDHx}(\mathrm{M})$ & SDHx(M) & $\mathrm{SDHx}(\mathrm{M})$ & $\mathrm{pHyp}(\mathrm{H})$ \\
\hline RNSH19 & PCC & FFPE & 41 & Left adrenal & Kolling & $S D H B$ & $\mathrm{SDHx}(\mathrm{H})$ & $\mathrm{SDH} x(\mathrm{H})$ & $\mathrm{SDHx}(\mathrm{H})$ & $\mathrm{pHyp}(\mathrm{H})$ \\
\hline VCB.01T & PCC & FFPE & 57 & Right adrenal & VCB & $R E T$ & RTK1(M) & RTK1(M) & RTK(H) & RTK(H) \\
\hline VCB.04T & PCC & FFPE & 24 & Bilateral & VCB & $V H L$ & RTK2(M) & $\mathrm{SDHx}(\mathrm{L})$ & $\mathrm{SDHx}(\mathrm{L})$ & $\mathrm{pHyp}(\mathrm{H})$ \\
\hline VCB.06T & PCC & FFPE & 17 & Right adrenal & VCB & $R E T$ & RTK1(H) & RTK1(H) & RTK(H) & RTK(H) \\
\hline VCB.07T & PCC & FFPE & 60 & Right adrenal & VCB & HRAS & RTK1(H) & RTK1(H) & RTK(H) & RTK(H) \\
\hline VCB.09T & PCC & FFPE & 76 & Left adrenal & VCB & $R E T$ & RTK2(H) & RTK3(H) & RTK(H) & RTK(H) \\
\hline VCB.10T & PCC & FFPE & 31 & Right adrenal & VCB & NF1 & RTK1(H) & RTK1(H) & RTK(H) & RTK(H) \\
\hline VCB.11T & PCC & FFPE & 56 & Right adrenal & VCB & NF1 & RTK3 $(\mathrm{H})$ & RTK3(H) & RTK(H) & RTK(H) \\
\hline VCB.13T & PCC & FFPE & 26 & Right adrenal & VCB & $V H L$ & VHL(M) & VHL(H) & VHL(H) & $\mathrm{pHyp}(\mathrm{H})$ \\
\hline VCB.14T & PCC & FFPE & 34 & Bilaterlal & VCB & $V H L$ & VHL(M) & VHL(M) & $\mathrm{VHL}(\mathrm{M})$ & pHyp(H) \\
\hline WM01 & PCC & Fresh & 7 & ND & $\mathrm{WCH}$ & $V H L$ & MAX-Like(L) & MAX-Like(L) & MAX-Like(L) & pHyp(M) \\
\hline WM02 & PCC & Fresh & 8 & ND & WCH & $V H L$ & MAX-Like(L) & MAX-Like(L) & MAX-Like(L) & pHyp(M) \\
\hline WM03 & PCC & FFPE & 3 & ND & $\mathrm{WCH}$ & $V H L$ & VHL(M) & $\mathrm{VHL}(\mathrm{H})$ & $\mathrm{VHL}(\mathrm{H})$ & pHyp(H) \\
\hline WM04 & PCC & FFPE & 13 & ND & $\mathrm{WCH}$ & $V H L$ & VHL(M) & VHL(H) & $\mathrm{VHL}(\mathrm{H})$ & $\mathrm{pHyp}(\mathrm{H})$ \\
\hline WM05 & PCC & FFPE & 8 & ND & $\mathrm{WCH}$ & $V H L$ & RTK2(M) & VHL(M) & VHL(M) & $\mathrm{pHyp}(\mathrm{H})$ \\
\hline WM06 & PCC & Fresh & 10 & ND & $\mathrm{WCH}$ & $V H L$ & $\mathrm{VHL}(\mathrm{H})$ & $\mathrm{VHL}(\mathrm{H})$ & $\mathrm{VHL}(\mathrm{H})$ & $\mathrm{pHyp}(\mathrm{H})$ \\
\hline WM07 & PGL & FFPE & 7 & ND & $\mathrm{WCH}$ & SDHB IHC Neg & $\mathrm{SDHx}(\mathrm{M})$ & $\mathrm{SDHx}(\mathrm{M})$ & $\mathrm{SDHx}(\mathrm{M})$ & $\mathrm{pHyp}(\mathrm{H})$ \\
\hline
\end{tabular}

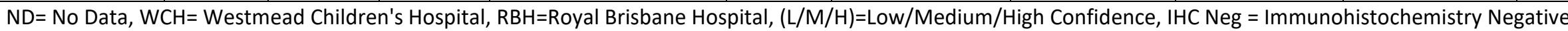


Appendix C Expression of classifier genes distinguishing the RTK1 class in the training set
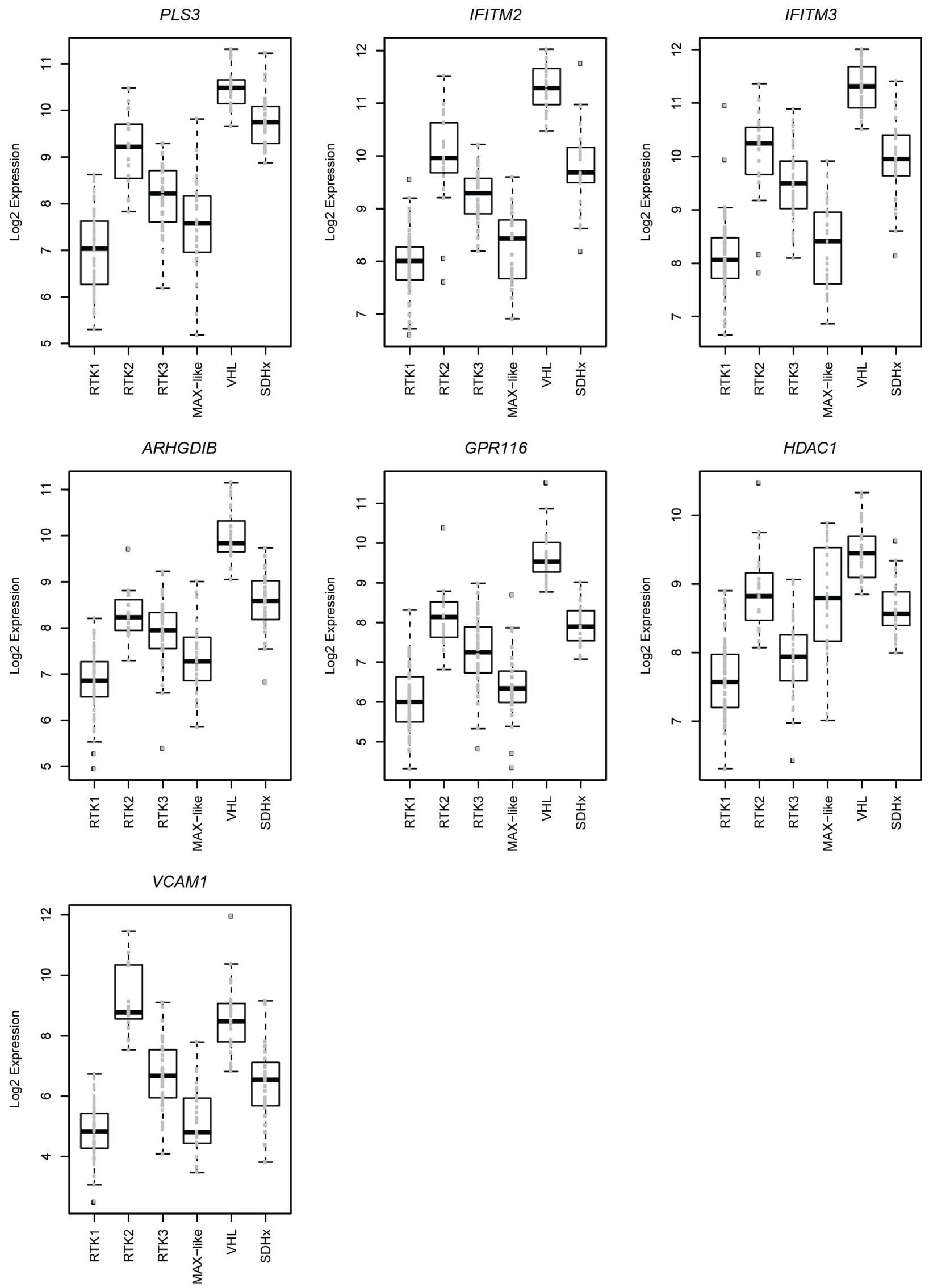
Appendix D Expression of classifier genes distinguishing the RTK2 class in the training set

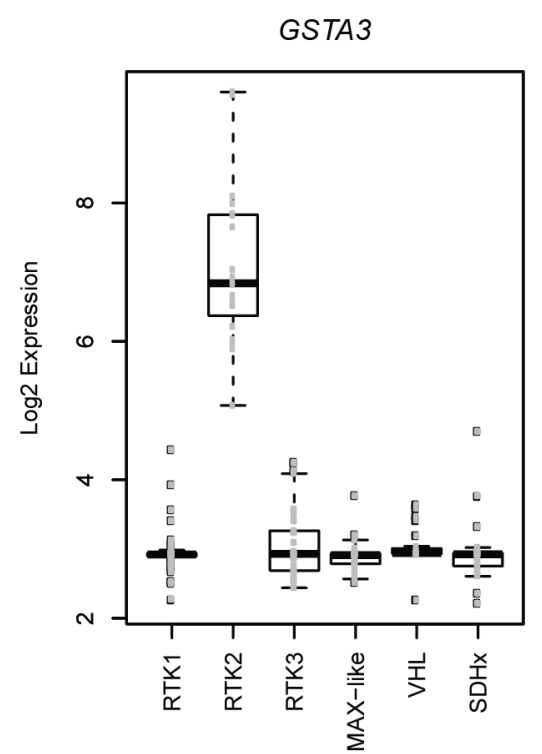

TST

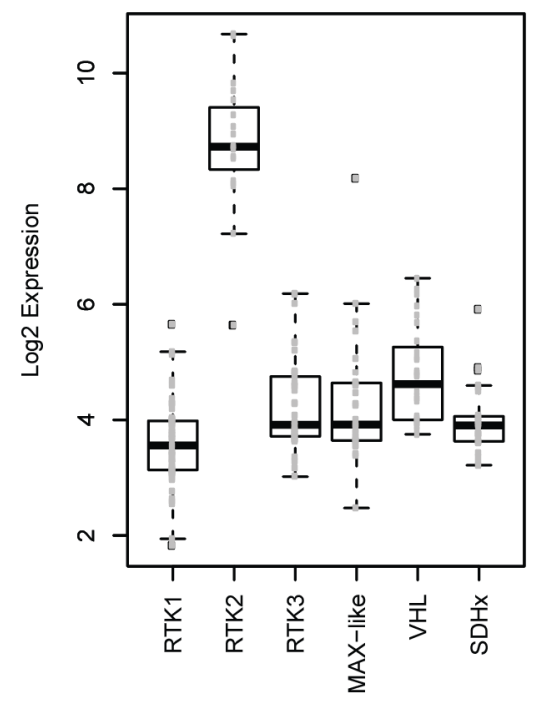

FDX1

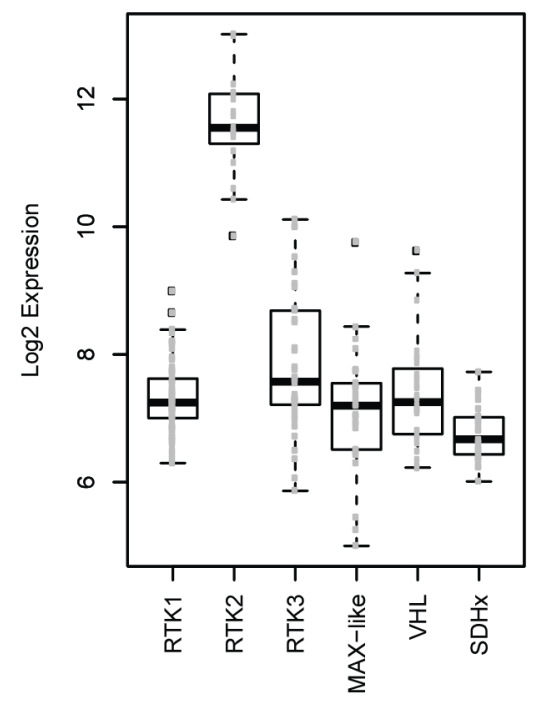

SULT2A1

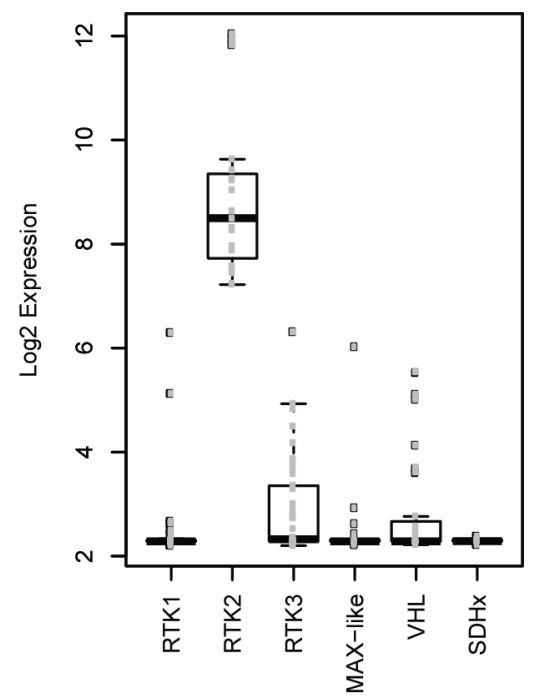

CYP11A1

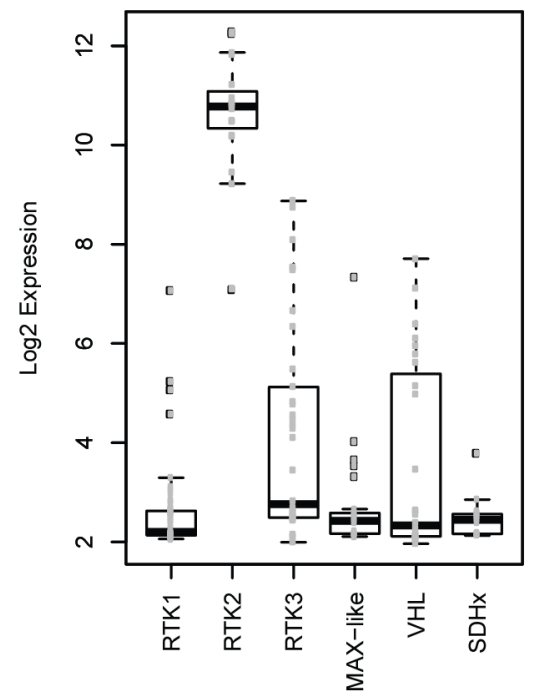

NR1H4

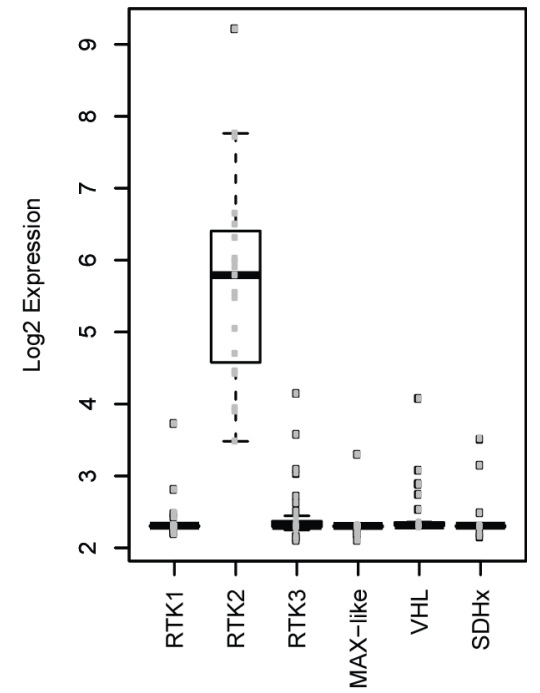

$R B B P 7$

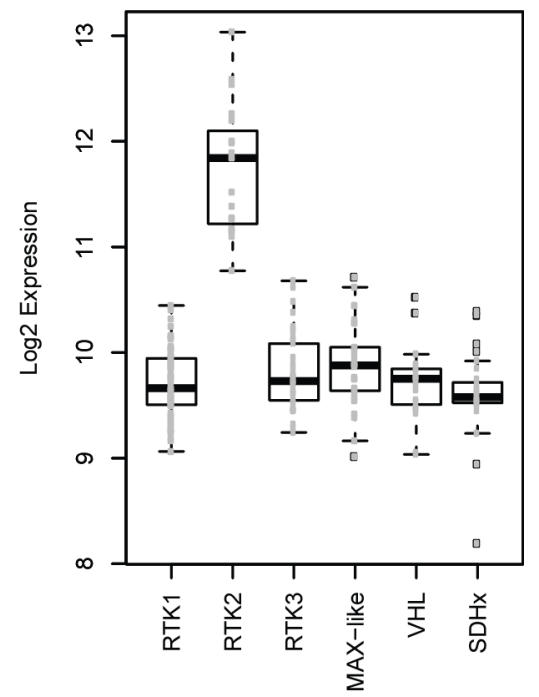


Appendix E Expression of classifier genes distinguishing the RTK3 class in the training set
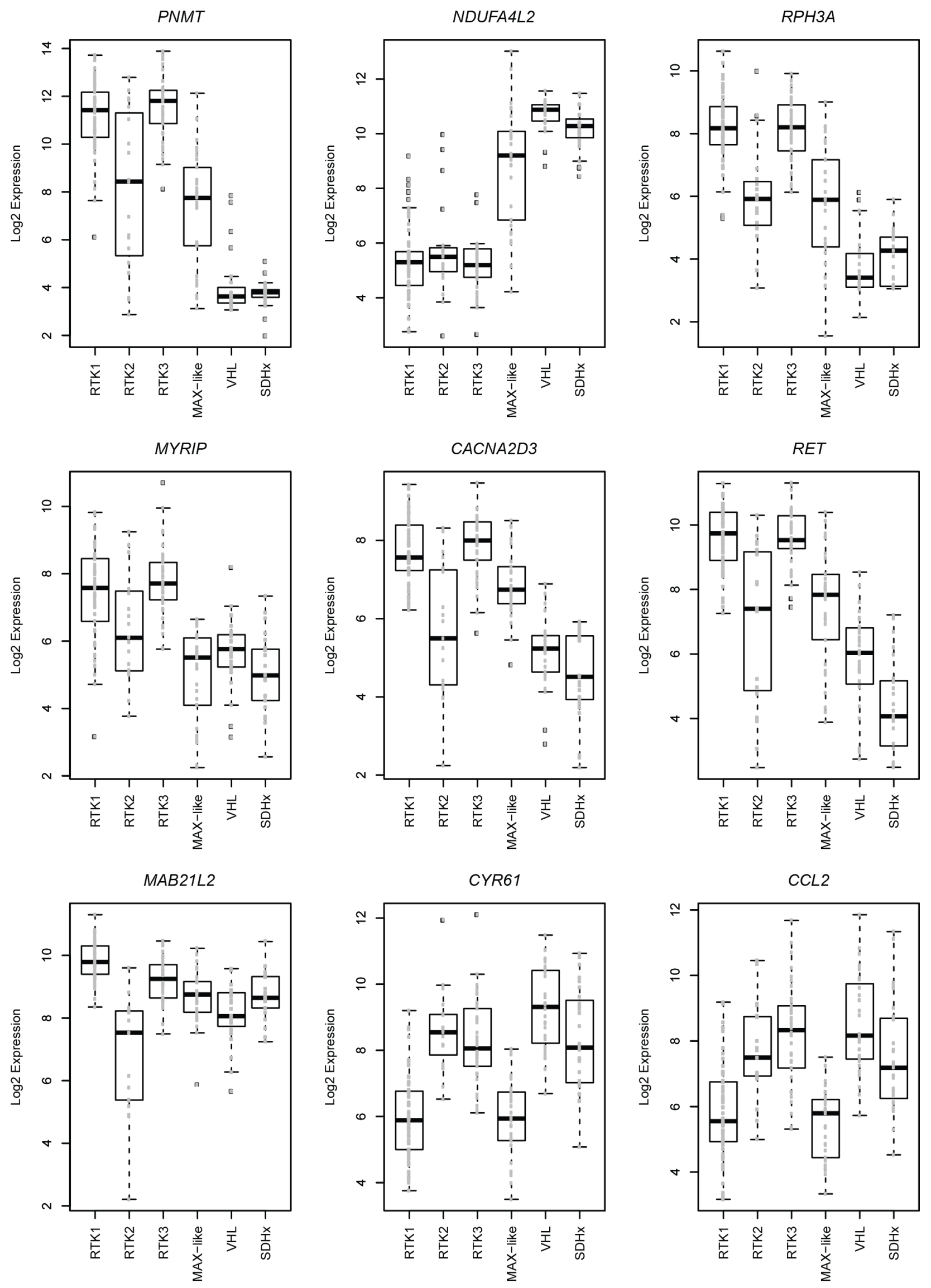
Appendix F Expression of classifier genes distinguishing the MAX-like class in the training set

TRIB2
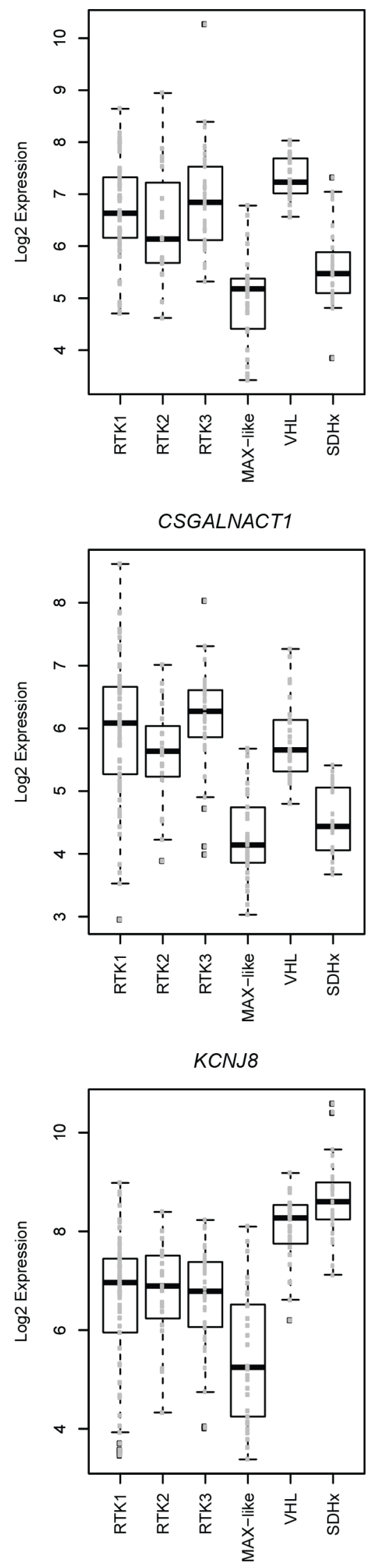

DUSP6

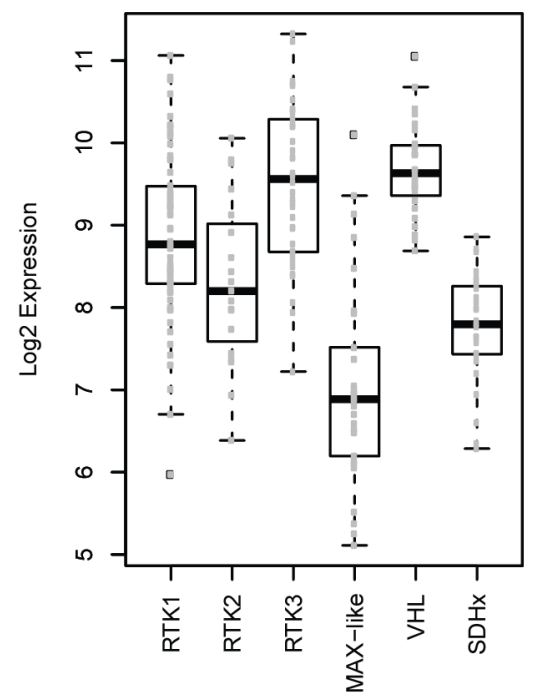

PFKFB3
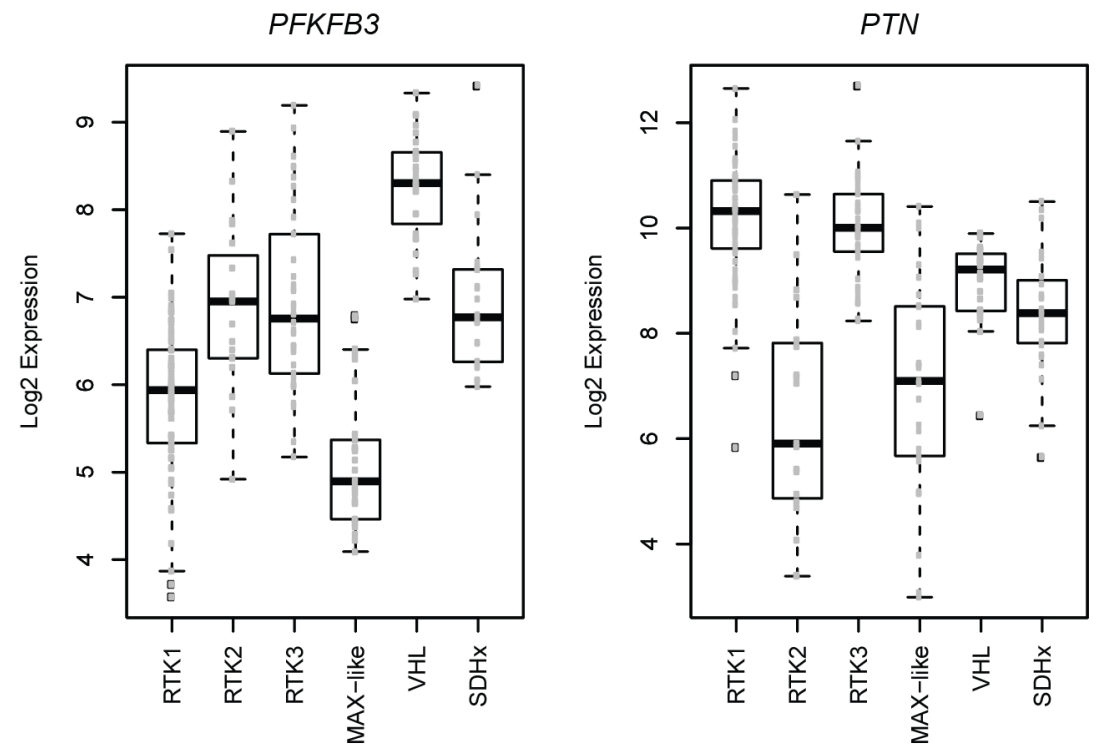
Appendix G Expression of classifier genes distinguishing the SDHx class in the training set

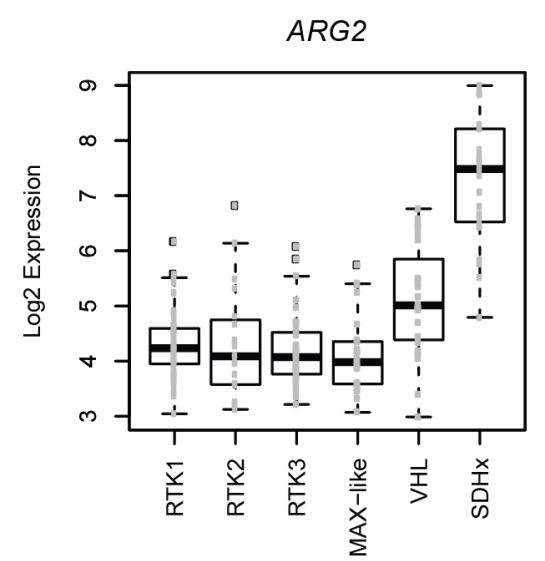

MAPK13
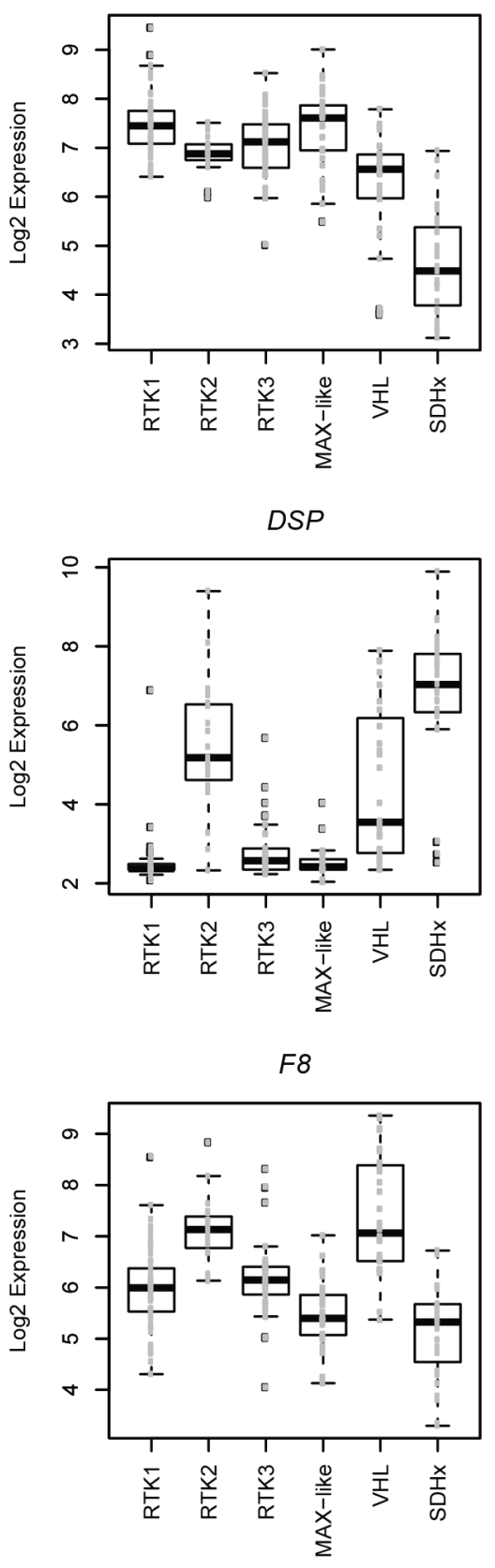

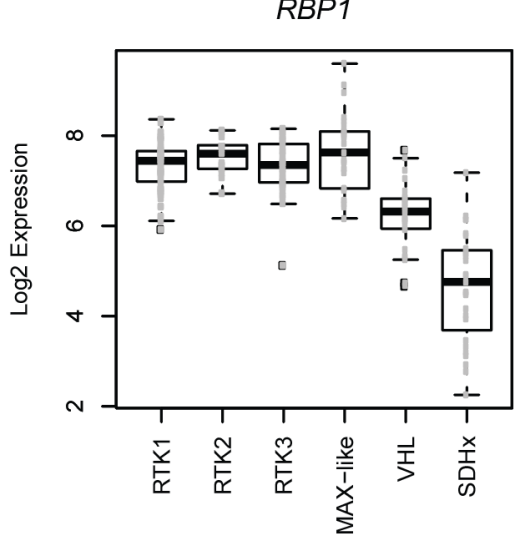

CRYBA2

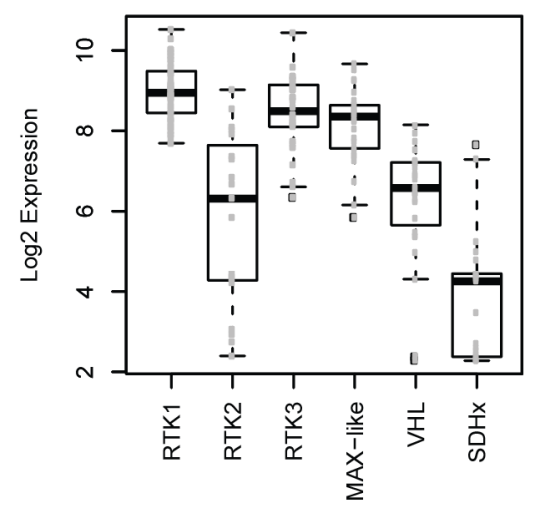

TRIB3

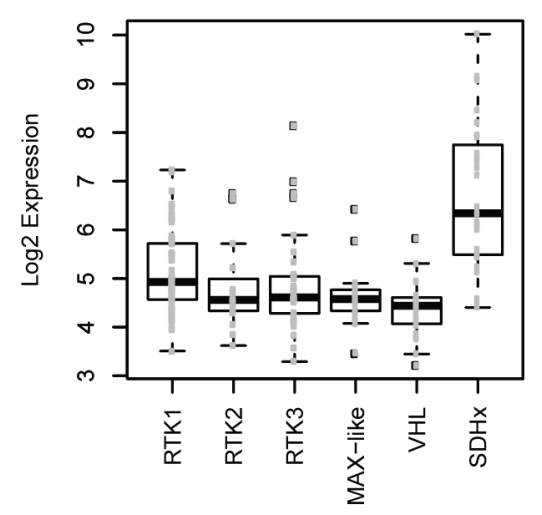

MID1IP1

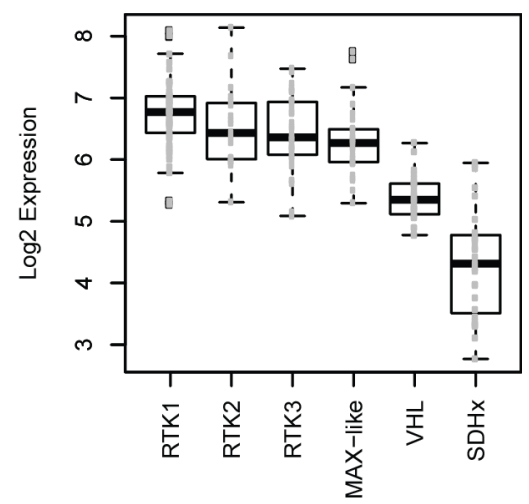

TBL $1 X$

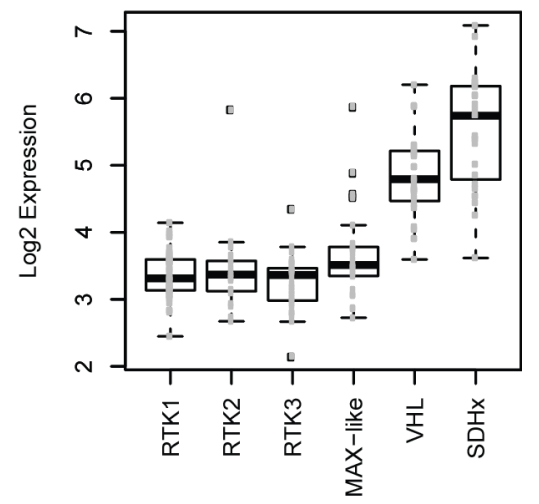

HSPB1

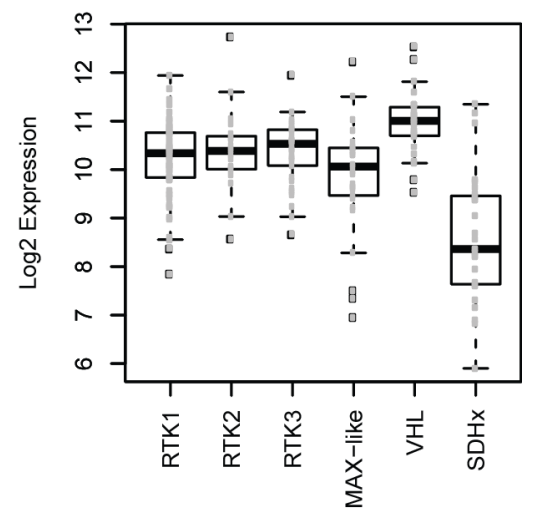


Appendix H Expression of classifier genes distinguishing the VHL class in the training set
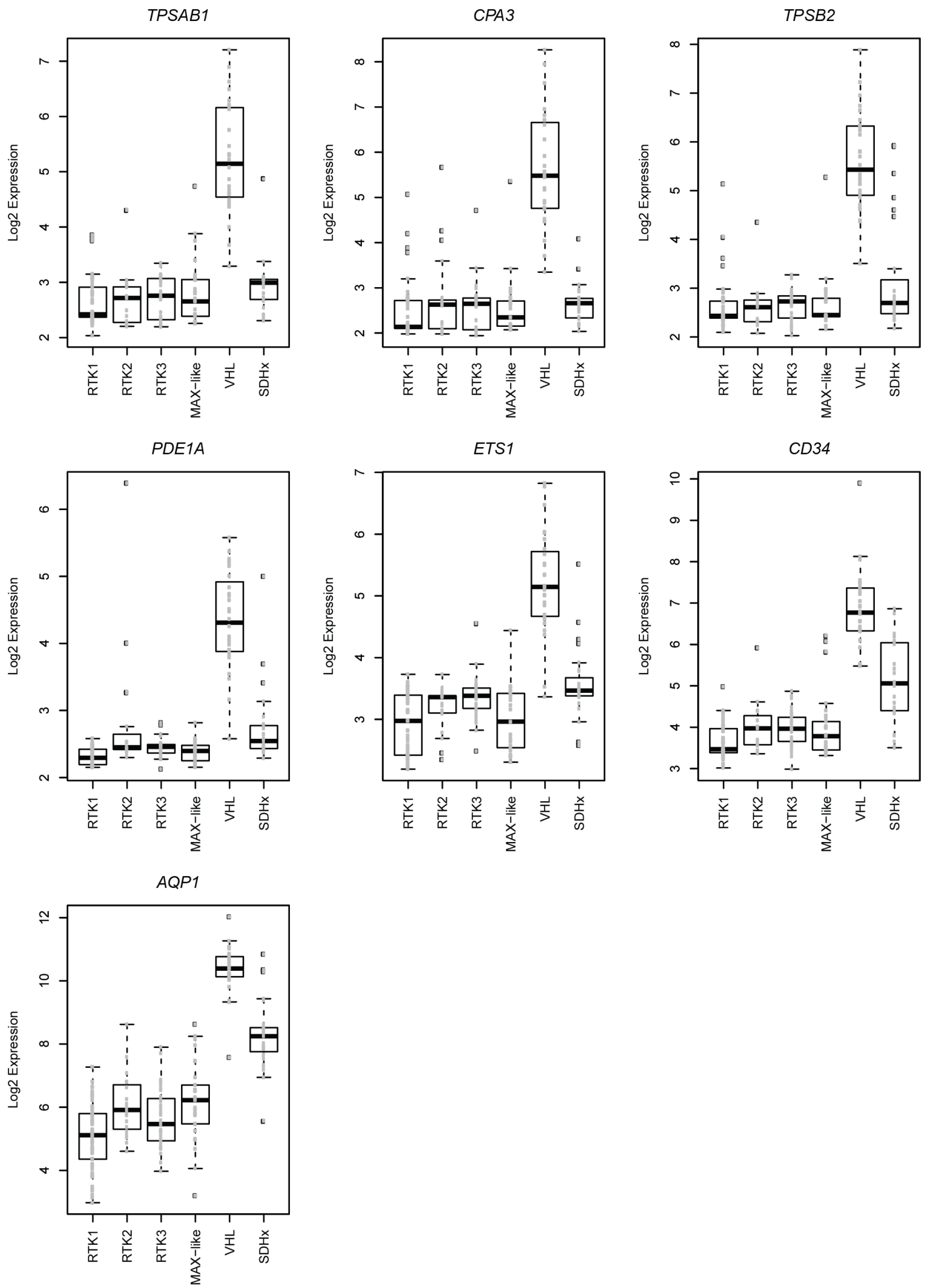
Appendix I Expression of Nanostring control genes in the training set
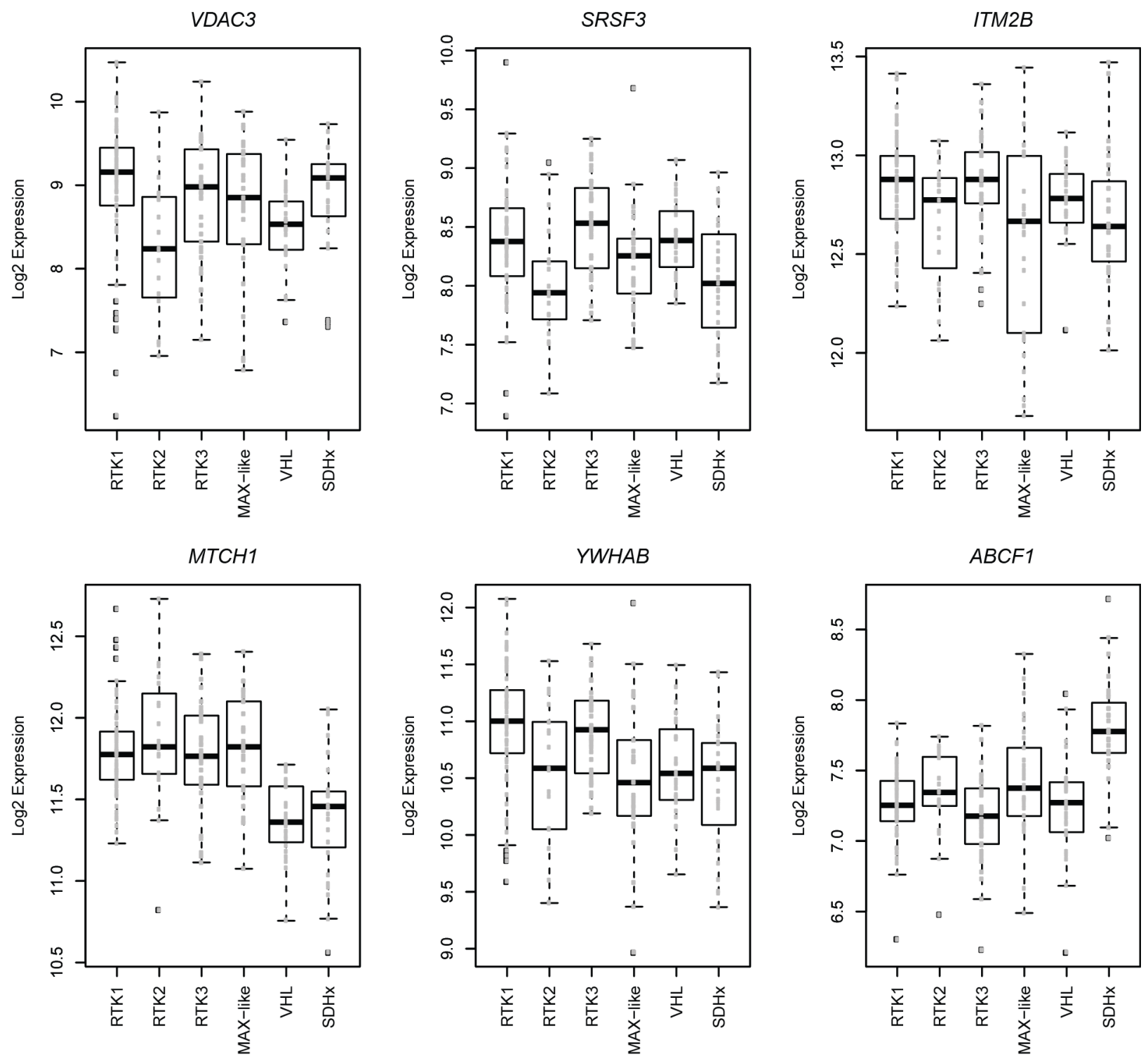


\section{References}

Abedalthagafi, M., Phillips, J. J., Kim, G. E., Mueller, S., Haas-Kogen, D. A., Marshall, R. E., . . Perry, A. (2013). The alternative lengthening of telomere phenotype is significantly associated with loss of ATRX expression in high-grade pediatric and adult astrocytomas: a multi-institutional study of 214 astrocytomas. Mod Pathol, 26(11), 1425-1432. doi:10.1038/modpathol.2013.90

Abermil, N., Guillaud-Bataille, M., Burnichon, N., Venisse, A., Manivet, P., Guignat, L., . . GimenezRoqueplo, A. P. (2012). TMEM127 screening in a large cohort of patients with pheochromocytoma and/or paraganglioma. J Clin Endocrinol Metab, 97(5), E805-809. doi:10.1210/jc.2011-3360

Agarwal, A., Mehrotra, P. K., Jain, M., Gupta, S. K., Mishra, A., Chand, G., . . Singh, U. (2010). Size of the tumor and pheochromocytoma of the adrenal gland scaled score (PASS): can they predict malignancy? World J Surg, 34(12), 3022-3028. doi:10.1007/s00268-010-0744-5

Agrawal, N., Jiao, Y., Sausen, M., Leary, R., Bettegowda, C., Roberts, N. J., . . Ball, D. W. (2013). Exomic sequencing of medullary thyroid cancer reveals dominant and mutually exclusive oncogenic mutations in RET and RAS. J Clin Endocrinol Metab, 98(2), E364-369. doi:10.1210/jc.20122703jc.2012-2703 [pii]

Alexandrov, L. B., Nik-Zainal, S., Wedge, D. C., Aparicio, S. A., Behjati, S., Biankin, A. V., ... Stratton, M. R. (2013). Signatures of mutational processes in human cancer. Nature, 500(7463), 415-421. doi:10.1038/nature12477 nature12477 [pii]

Alexandrov, L. B., Nik-Zainal, S., Wedge, D. C., Campbell, P. J., \& Stratton, M. R. (2013). Deciphering signatures of mutational processes operative in human cancer. Cell Rep, 3(1), 246-259. doi:10.1016/j.celrep.2012.12.008 S2211-1247(12)00433-0 [pii]

Allegra, C. J., Jessup, J. M., Somerfield, M. R., Hamilton, S. R., Hammond, E. H., Hayes, D. F., . . Schilsky, R. L. (2009). American Society of Clinical Oncology provisional clinical opinion: testing for KRAS gene mutations in patients with metastatic colorectal carcinoma to predict response to antiepidermal growth factor receptor monoclonal antibody therapy. J Clin Oncol, 27(12), 2091-2096. doi:10.1200/JCO.2009.21.9170

Amarasinghe, K. C., Li, J., Hunter, S. M., Ryland, G. L., Cowin, P. A., Campbell, I. G., \& Halgamuge, S. K. (2014). Inferring copy number and genotype in tumour exome data. BMC Genomics, 15, 732. doi:10.1186/1471-2164-15-732

Andreasson, A., Kiss, N. B., Caramuta, S., Sulaiman, L., Svahn, F., Backdahl, M., . . Larsson, C. (2013). The VHL gene is epigenetically inactivated in pheochromocytomas and abdominal paragangliomas. Epigenetics, 8(12), 1347-1354. doi:10.4161/epi.26686

Arighi, E., Alberti, L., Torriti, F., Ghizzoni, S., Rizzetti, M. G., Pelicci, G., . . Borrello, M. G. (1997). Identification of Shc docking site on Ret tyrosine kinase. Oncogene, 14(7), 773-782. doi:10.1038/sj.onc.1200896

Arighi, E., Borrello, M. G., \& Sariola, H. (2005). RET tyrosine kinase signaling in development and cancer. Cytokine Growth Factor Rev, 16(4-5), 441-467. doi:S1359-6101(05)00074-2 [pii] 10.1016/j.cytogfr.2005.05.010

Asai, N., Iwashita, T., Matsuyama, M., \& Takahashi, M. (1995). Mechanism of activation of the ret protooncogene by multiple endocrine neoplasia 2A mutations. Mol Cell Biol, 15(3), 1613-1619.

Asai, N., Murakami, H., Iwashita, T., \& Takahashi, M. (1996). A mutation at tyrosine 1062 in MEN2A-Ret and MEN2B-Ret impairs their transforming activity and association with shc adaptor proteins. $J$ Biol Chem, 271(30), 17644-17649. 
Astuti, D., Ricketts, C. J., Chowdhury, R., McDonough, M. A., Gentle, D., Kirby, G., . . Maher, E. R. (2011). Mutation analysis of HIF prolyl hydroxylases (PHD/EGLN) in individuals with features of phaeochromocytoma and renal cell carcinoma susceptibility. Endocr Relat Cancer, 18(1), 73-83. doi:10.1677/ERC-10-0113 ERC-10-0113 [pii]

Avery, O. T., Macleod, C. M., \& McCarty, M. (1944). Studies on the Chemical Nature of the Substance Inducing Transformation of Pneumococcal Types : Induction of Transformation by a Desoxyribonucleic Acid Fraction Isolated from Pneumococcus Type lii. J Exp Med, 79(2), 137-158.

Ayala-Ramirez, M., Feng, L., Johnson, M. M., Ejaz, S., Habra, M. A., Rich, T., . . J Jimenez, C. (2011). Clinical risk factors for malignancy and overall survival in patients with pheochromocytomas and sympathetic paragangliomas: primary tumor size and primary tumor location as prognostic indicators. J Clin Endocrinol Metab, 96(3), 717-725. doi:10.1210/jc.2010-1946 jc.2010-1946 [pii]

Ayer, D. E., Kretzner, L., \& Eisenman, R. N. (1993). Mad: a heterodimeric partner for Max that antagonizes Myc transcriptional activity. Cell, 72(2), 211-222.

Baguet, J. P., Hammer, L., Mazzuco, T. L., Chabre, O., Mallion, J. M., Sturm, N., \& Chaffanjon, P. (2004). Circumstances of discovery of phaeochromocytoma: a retrospective study of 41 consecutive patients. Eur J Endocrinol, 150(5), 681-686.

Ballester, R., Marchuk, D., Boguski, M., Saulino, A., Letcher, R., Wigler, M., \& Collins, F. (1990). The NF1 locus encodes a protein functionally related to mammalian GAP and yeast IRA proteins. Cell, 63(4), 851-859. doi:0092-8674(90)90151-4 [pii]

Barnes, D. R., \& Antoniou, A. C. (2012). Unravelling modifiers of breast and ovarian cancer risk for BRCA1 and BRCA2 mutation carriers: update on genetic modifiers. J Intern Med, 271(4), 331-343. doi:10.1111/j.1365-2796.2011.02502.x

Bashashati, A., Ha, G., Tone, A., Ding, J., Prentice, L. M., Roth, A., ... Shah, S. P. (2013). Distinct evolutionary trajectories of primary high-grade serous ovarian cancers revealed through spatial mutational profiling. J Pathol, 231(1), 21-34. doi:10.1002/path.4230

Baylin, S. B. (2005). DNA methylation and gene silencing in cancer. Nat Clin Pract Oncol, 2 Suppl 1, S4-11. doi:10.1038/ncponc0354

Beard, C. M., Sheps, S. G., Kurland, L. T., Carney, J. A., \& Lie, J. T. (1983). Occurrence of pheochromocytoma in Rochester, Minnesota, 1950 through 1979. Mayo Clin Proc, 58(12), 802804.

Benn, D. E., Richardson, A. L., Marsh, D. J., \& Robinson, B. G. (2006). Genetic testing in pheochromocytoma- and paraganglioma-associated syndromes. Ann N Y Acad Sci, 1073, 104111. doi:1073/1/104 [pii] 10.1196/annals.1353.011

Benn, D. E., Robinson, B. G., \& Clifton-Bligh, R. J. (2015). 15 YEARS OF PARAGANGLIOMA: Clinical manifestations of paraganglioma syndromes types 1-5. Endocr Relat Cancer, 22(4), T91-103. doi:10.1530/ERC-15-0268

Bento, C., Percy, M. J., Gardie, B., Maia, T. M., van Wijk, R., Perrotta, S., . . Consortium, E. C. E. (2014). Genetic basis of congenital erythrocytosis: mutation update and online databases. Hum Mutat, 35(1), 15-26. doi:10.1002/humu.22448

Beristain, E., Vicente, M. A., Guerra, I., Gutierrez-Corres, F. B., Garin, I., \& Perez de Nanclares, G. (2013). Disomy as the genetic underlying mechanisms of loss of heterozigosity in SDHD-paragangliomas. J Clin Endocrinol Metab, 98(5), E1012-1016. doi:10.1210/jc.2012-4083

Bernards, R., \& Weinberg, R. A. (2002). A progression puzzle. Nature, 418(6900), 823. doi:10.1038/418823a

Bettegowda, C., Sausen, M., Leary, R. J., Kinde, I., Wang, Y., Agrawal, N., . . Diaz, L. A., Jr. (2014). Detection of circulating tumor DNA in early- and late-stage human malignancies. Sci Trans/ Med, 6(224), 224ra224. doi:10.1126/scitranslmed.3007094 
Bleeker, F. E., Lamba, S., Leenstra, S., Troost, D., Hulsebos, T., Vandertop, W. P., . . Bardelli, A. (2009). IDH1 mutations at residue p.R132 (IDH1(R132)) occur frequently in high-grade gliomas but not in other solid tumors. Hum Mutat, 30(1), 7-11. doi:10.1002/humu.20937

Borrello, M. G., Smith, D. P., Pasini, B., Bongarzone, I., Greco, A., Lorenzo, M. J., . . . et al. (1995). RET activation by germline MEN2A and MEN2B mutations. Oncogene, 11(11), 2419-2427.

Boutros, P. C., Fraser, M., Harding, N. J., de Borja, R., Trudel, D., Lalonde, E., . . Bristow, R. G. (2015). Spatial genomic heterogeneity within localized, multifocal prostate cancer. Nat Genet. doi:10.1038/ng.3315

Boveri, T. (2008). Concerning the origin of malignant tumours by Theodor Boveri. Translated and annotated by Henry Harris. J Cell Sci, 121 Supp/ 1, 1-84. doi:10.1242/jcs.025742

Boyd, K. P., Korf, B. R., \& Theos, A. (2009). Neurofibromatosis type 1. J Am Acad Dermatol, 61(1), 1-14; quiz 15-16. doi:S0190-9622(09)00405-8 [pii] 10.1016/j.jaad.2008.12.051

Bozic, I., Reiter, J. G., Allen, B., Antal, T., Chatterjee, K., Shah, P., . . Nowak, M. A. (2013). Evolutionary dynamics of cancer in response to targeted combination therapy. Elife, 2, e00747. doi:10.7554/eLife.00747

Brandi, M. L., Gagel, R. F., Angeli, A., Bilezikian, J. P., Beck-Peccoz, P., Bordi, C., . . Marx, S. J. (2001). Guidelines for diagnosis and therapy of MEN type 1 and type 2. J Clin Endocrinol Metab, 86(12), 5658-5671.

Brito, J. P., Asi, N., Bancos, I., Gionfriddo, M. R., Zeballos-Palacios, C. L., Leppin, A. L., . . Murad, M. H. (2014). Testing for germline mutations in sporadic pheochromocytoma/paraganglioma: a systematic review. Clin Endocrinol (Oxf). doi:10.1111/cen.12530

Burnichon, N., Briere, J. J., Libe, R., Vescovo, L., Riviere, J., Tissier, F., . . . Gimenez-Roqueplo, A. P. (2010). SDHA is a tumor suppressor gene causing paraganglioma. Hum Mol Genet, 19(15), 3011-3020. doi:10.1093/hmg/ddq206

Burnichon, N., Buffet, A., Parfait, B., Letouze, E., Laurendeau, I., Loriot, C., . . Gimenez-Roqueplo, A. P. (2012). Somatic NF1 Inactivation is a Frequent Event in Sporadic Pheochromocytoma. Hum Mol Genet. doi:dds374 [pii] 10.1093/hmg/dds374

Burnichon, N., Cascon, A., Schiavi, F., Morales, N. P., Comino-Mendez, I., Abermil, N., ... Robledo, M. (2012). MAX Mutations Cause Hereditary and Sporadic Pheochromocytoma and Paraganglioma. Clin Cancer Res, 18(10), 2828-2837. doi:1078-0432.CCR-12-0160 [pii] 10.1158/1078-0432.CCR$12-0160$

Burnichon, N., Vescovo, L., Amar, L., Libe, R., de Reynies, A., Venisse, A., . . Gimenez-Roqueplo, A. P. (2011). Integrative genomic analysis reveals somatic mutations in pheochromocytoma and paraganglioma. Hum Mol Genet, 20(20), 3974-3985. doi:ddr324 [pii] 10.1093/hmg/ddr324

Burotto, M., Chiou, V. L., Lee, J. M., \& Kohn, E. C. (2014). The MAPK pathway across different malignancies: a new perspective. Cancer, 120(22), 3446-3456. doi:10.1002/cncr.28864

Bussolati, G., Annaratone, L., \& Maletta, F. (2015). The pre-analytical phase in surgical pathology. Recent Results Cancer Res, 199, 1-13. doi:10.1007/978-3-319-13957-9_1

Calcagno, D. Q., Leal, M. F., Assumpcao, P. P., Smith, M. A., \& Burbano, R. R. (2008). MYC and gastric adenocarcinoma carcinogenesis. World J Gastroenterol, 14(39), 5962-5968.

Cancer Genome Atlas Research, N., Weinstein, J. N., Collisson, E. A., Mills, G. B., Shaw, K. R., Ozenberger, B. A., . . Stuart, J. M. (2013). The Cancer Genome Atlas Pan-Cancer analysis project. Nat Genet, 45(10), 1113-1120. doi:10.1038/ng.2764

Carrasquillo, J. A., \& Chen, C. C. (2010). Molecular imaging of neuroendocrine tumors. Semin Oncol, 37(6), 662-679. doi:S0093-7754(10)00185-5 [pii] 10.1053/j.seminoncol.2010.10.015

Cascon, A., Comino-Mendez, I., Curras-Freixes, M., de Cubas, A. A., Contreras, L., Richter, S., . . Robledo, M. (2015). Whole-Exome Sequencing Identifies MDH2 as a New Familial Paraganglioma Gene. J Natl Cancer Inst, 107(5). doi:10.1093/jnci/djv053 
Cascon, A., Ruiz-Llorente, S., Fraga, M. F., Leton, R., Telleria, D., Sastre, J., . . Robledo, M. (2004). Genetic and epigenetic profile of sporadic pheochromocytomas. J Med Genet, 41(3), e30.

Castro-Vega, L. J., Buffet, A., de Cubas, A. A., Cascon, A., Menara, M., Khalifa, E., . . . Gimenez-Roqueplo, A. P. (2013). Germline mutations in FH confer predisposition to malignant pheochromocytomas and paragangliomas. Hum Mol Genet. doi:ddt639 [pii] 10.1093/hmg/ddt639

Castro-Vega, L. J., Lepoutre-Lussey, C., Gimenez-Roqueplo, A. P., \& Favier, J. (2015). Rethinking pheochromocytomas and paragangliomas from a genomic perspective. Oncogene. doi:10.1038/onc.2015.172

Castro-Vega, L. J., Letouze, E., Burnichon, N., Buffet, A., Disderot, P. H., Khalifa, E., . . GimenezRoqueplo, A. P. (2015). Multi-omics analysis defines core genomic alterations in pheochromocytomas and paragangliomas. Nat Commun, 6, 6044. doi:10.1038/ncomms7044

Cervera, A. M., Bayley, J. P., Devilee, P., \& McCreath, K. J. (2009). Inhibition of succinate dehydrogenase dysregulates histone modification in mammalian cells. Mol Cancer, 8, 89. doi:10.1186/14764598-8-89

Chandrasekharappa, S. C., Guru, S. C., Manickam, P., Olufemi, S. E., Collins, F. S., Emmert-Buck, M. R., . . Marx, S. J. (1997). Positional cloning of the gene for multiple endocrine neoplasia-type 1. Science, 276(5311), 404-407.

Chen, Y., \& Olopade, O. I. (2008). MYC in breast tumor progression. Expert Rev Anticancer Ther, 8(10), 1689-1698. doi:10.1586/14737140.8.10.1689

Chen, Z. X., Wallis, K., Fell, S. M., Sobrado, V. R., Hemmer, M. C., Ramskold, D., .. Schlisio, S. (2014). RNA helicase $A$ is a downstream mediator of KIF1Bbeta tumor-suppressor function in neuroblastoma. Cancer Discov, 4(4), 434-451. doi:10.1158/2159-8290.CD-13-0362

Chi, J. T., Wang, Z., Nuyten, D. S., Rodriguez, E. H., Schaner, M. E., Salim, A., . . Brown, P. O. (2006). Gene expression programs in response to hypoxia: cell type specificity and prognostic significance in human cancers. PLoS Med, 3(3), e47. doi:10.1371/journal.pmed.0030047

Chow, M. T., Moller, A., \& Smyth, M. J. (2012). Inflammation and immune surveillance in cancer. Semin Cancer Biol, 22(1), 23-32. doi:10.1016/j.semcancer.2011.12.004

Ciampi, R., Romei, C., Cosci, B., Vivaldi, A., Bottici, V., Renzini, G., . . Elisei, R. (2012). Chromosome 10 and RET gene copy number alterations in hereditary and sporadic Medullary Thyroid Carcinoma. Mol Cell Endocrinol, 348(1), 176-182. doi:10.1016/j.mce.2011.08.004

Cibulskis, K., Lawrence, M. S., Carter, S. L., Sivachenko, A., Jaffe, D., Sougnez, C., . . Getz, G. (2013). Sensitive detection of somatic point mutations in impure and heterogeneous cancer samples. Nat Biotechnol. doi:10.1038/nbt.2514 nbt.2514 [pii]

Clark, G. R., Sciacovelli, M., Gaude, E., Walsh, D. M., Kirby, G., Simpson, M. A., . . Maher, E. R. (2014). Germline FH mutations presenting with pheochromocytoma. J Clin Endocrinol Metab, jc20141659. doi:10.1210/jc.2014-1659

Cleary, A. S., Leonard, T. L., Gestl, S. A., \& Gunther, E. J. (2014). Tumour cell heterogeneity maintained by cooperating subclones in Wnt-driven mammary cancers. Nature, 508(7494), 113-117. doi:10.1038/nature13187

Clifford, S. C., Cockman, M. E., Smallwood, A. C., Mole, D. R., Woodward, E. R., Maxwell, P. H., ... Maher, E. R. (2001). Contrasting effects on HIF-1alpha regulation by disease-causing PVHL mutations correlate with patterns of tumourigenesis in von Hippel-Lindau disease. Hum Mol Genet, 10(10), 1029-1038.

Comino-Mendez, I., de Cubas, A. A., Bernal, C., Alvarez-Escola, C., Sanchez-Malo, C., Ramirez-Tortosa, C. L., ... Cascon, A. (2013). Tumoral EPAS1 (HIF2A) mutations explain sporadic pheochromocytoma and paraganglioma in the absence of erythrocytosis. Hum Mol Genet. doi:ddt069 [pii] $10.1093 / \mathrm{hmg} / \mathrm{ddt069}$ 
Comino-Mendez, I., Gracia-Aznarez, F. J., Schiavi, F., Landa, I., Leandro-Garcia, L. J., Leton, R., .. . Cascon, A. (2011). Exome sequencing identifies MAX mutations as a cause of hereditary pheochromocytoma. Nat Genet, 43(7), 663-667. doi:10.1038/ng.861 ng.861 [pii]

Cooke, S. L., Temple, J., Macarthur, S., Zahra, M. A., Tan, L. T., Crawford, R. A., . . Brenton, J. D. (2011). Intra-tumour genetic heterogeneity and poor chemoradiotherapy response in cervical cancer. $\mathrm{Br}$ J Cancer, 104(2), 361-368. doi:10.1038/sj.bjc.6605971

Corn, P. G., Ricci, M. S., Scata, K. A., Arsham, A. M., Simon, M. C., Dicker, D. T., \& El-Deiry, W. S. (2005). Mxi1 is induced by hypoxia in a HIF-1-dependent manner and protects cells from c-Myc-induced apoptosis. Cancer Biol Ther, 4(11), 1285-1294.

Crona, J., Backman, S., Maharjan, R., Mayrhofer, M., Stalberg, P., Isakson, A., . . Bjorklund, P. (2015). Spatio-temporal heterogeneity characterizes the genetic landscape of pheochromocytoma and defines early events in tumourigenesis. Clin Cancer Res. doi:10.1158/1078-0432.CCR-14-2854

Crona, J., Delgado Verdugo, A., Maharjan, R., Stalberg, P., Granberg, D., Hellman, P., \& Bjorklund, P. (2013). Somatic Mutations in H-RAS in Sporadic Pheochromocytoma and Paraganglioma Identified by Exome Sequencing. J Clin Endocrinol Metab. doi:jc.2012-4257 [pii] 10.1210/jc.20124257

Crona, J., Nordling, M., Maharjan, R., Granberg, D., Stalberg, P., Hellman, P., \& Bjorklund, P. (2014). Integrative genetic characterization and phenotype correlations in pheochromocytoma and paraganglioma tumours. PLoS One, 9(1), e86756. doi:10.1371/journal.pone.0086756 PONE-D13-30598 [pii]

Crowley, E., Di Nicolantonio, F., Loupakis, F., \& Bardelli, A. (2013). Liquid biopsy: monitoring cancergenetics in the blood. Nat Rev Clin Oncol, 10(8), 472-484. doi:10.1038/nrclinonc.2013.110

Cryer, P. E. (1980). Physiology and pathophysiology of the human sympathoadrenal neuroendocrine system. N Engl J Med, 303(8), 436-444. doi:10.1056/NEJM198008213030806

d'Herbomez, M., Forzy, G., Bauters, C., Tierny, C., Pigny, P., Carnaille, B., . . Rouaix, N. (2007). An analysis of the biochemical diagnosis of 66 pheochromocytomas. Eur J Endocrinol, 156(5), 569575. doi:10.1530/EJE-06-0640

Dahia, P. L. (2014). Pheochromocytoma and paraganglioma pathogenesis: learning from genetic heterogeneity. Nat Rev Cancer, 14(2), 108-119. doi:10.1038/nrc3648 nrc3648 [pii]

Dahia, P. L., Ross, K. N., Wright, M. E., Hayashida, C. Y., Santagata, S., Barontini, M., . . Stiles, C. D. (2005). A HIF1alpha regulatory loop links hypoxia and mitochondrial signals in pheochromocytomas. PLoS Genet, 1(1), 72-80. doi:10.1371/journal.pgen.0010008

Dang, C. V., Kim, J. W., Gao, P., \& Yustein, J. (2008). The interplay between MYC and HIF in cancer. Nat Rev Cancer, 8(1), 51-56. doi:10.1038/nrc2274

Dannenberg, H., Speel, E. J., Zhao, J., Saremaslani, P., van Der Harst, E., Roth, J., . . . de Krijger, R. R. (2000). Losses of chromosomes $1 p$ and $3 q$ are early genetic events in the development of sporadic pheochromocytomas. Am J Pathol, 157(2), 353-359. doi:S0002-9440(10)64547-6 [pii] 10.1016/S0002-9440(10)64547-6

Daston, M. M., Scrable, H., Nordlund, M., Sturbaum, A. K., Nissen, L. M., \& Ratner, N. (1992). The protein product of the neurofibromatosis type 1 gene is expressed at highest abundance in neurons, Schwann cells, and oligodendrocytes. Neuron, 8(3), 415-428. doi:0896-6273(92)90270-N [pii]

Davies, H., Bignell, G. R., Cox, C., Stephens, P., Edkins, S., Clegg, S., . . Futreal, P. A. (2002). Mutations of the BRAF gene in human cancer. Nature, 417(6892), 949-954. doi:10.1038/nature00766

De La Fuente, R., Viveiros, M. M., Wigglesworth, K., \& Eppig, J. J. (2004). ATRX, a member of the SNF2 family of helicase/ATPases, is required for chromosome alignment and meiotic spindle organization in metaphase II stage mouse oocytes. Dev Biol, 272(1), 1-14. doi:10.1016/j.ydbio.2003.12.012 
de Wailly, P., Oragano, L., Rade, F., Beaulieu, A., Arnault, V., Levillain, P., \& Kraimps, J. L. (2012). Malignant pheochromocytoma: new malignancy criteria. Langenbecks Arch Surg, 397(2), 239246. doi:10.1007/s00423-011-0850-3

Dekkers, M. P., Nikoletopoulou, V., \& Barde, Y. A. (2013). Cell biology in neuroscience: Death of developing neurons: new insights and implications for connectivity. J Cell Biol, 203(3), 385-393. doi:10.1083/jcb.201306136

Denorme, M., Yon, L., Roux, C., Gonzalez, B. J., Baudin, E., Anouar, Y., \& Dubessy, C. (2014). Both sunitinib and sorafenib are effective treatments for pheochromocytoma in a xenograft model. Cancer Lett, 352(2), 236-244. doi:10.1016/j.canlet.2014.07.005

Diehl, F., Schmidt, K., Choti, M. A., Romans, K., Goodman, S., Li, M., . . Diaz, L. A., Jr. (2008). Circulating mutant DNA to assess tumor dynamics. Nat Med, 14(9), 985-990. doi:10.1038/nm.1789

Diolaiti, D., McFerrin, L., Carroll, P. A., \& Eisenman, R. N. (2014). Functional interactions among members of the MAX and MLX transcriptional network during oncogenesis. Biochim Biophys Acta. doi:10.1016/j.bbagrm.2014.05.016

Druce, M. R., Kaltsas, G. A., Fraenkel, M., Gross, D. J., \& Grossman, A. B. (2009). Novel and evolving therapies in the treatment of malignant phaeochromocytoma: experience with the mTOR inhibitor everolimus (RAD001). Horm Metab Res, 41(9), 697-702. doi:10.1055/s-0029-1220687

Du, W., Wang, S., Zhou, Q., Li, X., Chu, J., Chang, Z., . . Y Yu, J. (2013). ADAMTS9 is a functional tumor suppressor through inhibiting AKT/mTOR pathway and associated with poor survival in gastric cancer. Oncogene, 32(28), 3319-3328. doi:10.1038/onc.2012.359

Edstrom, E., Mahlamaki, E., Nord, B., Kjellman, M., Karhu, R., Hoog, A., . . Larsson, C. (2000). Comparative genomic hybridization reveals frequent losses of chromosomes $1 p$ and $3 q$ in pheochromocytomas and abdominal paragangliomas, suggesting a common genetic etiology. Am J Pathol, 156(2), 651-659. doi:S0002-9440(10)64769-4 [pii] 10.1016/S0002-9440(10)64769-4

Eisenhofer, G., Bornstein, S. R., Brouwers, F. M., Cheung, N. K., Dahia, P. L., de Krijger, R. R., .. Young, W. F., Jr. (2004). Malignant pheochromocytoma: current status and initiatives for future progress. Endocr Relat Cancer, 11(3), 423-436.

Eisenhofer, G., Lenders, J. W., Siegert, G., Bornstein, S. R., Friberg, P., Milosevic, D., . . Pacak, K. (2012). Plasma methoxytyramine: a novel biomarker of metastatic pheochromocytoma and paraganglioma in relation to established risk factors of tumour size, location and SDHB mutation status. Eur J Cancer, 48(11), 1739-1749. doi:10.1016/j.ejca.2011.07.016

Eisenhofer, G., Rundquist, B., Aneman, A., Friberg, P., Dakak, N., Kopin, I. J., . . Lenders, J. W. (1995). Regional release and removal of catecholamines and extraneuronal metabolism to metanephrines. J Clin Endocrinol Metab, 80(10), 3009-3017. doi:10.1210/jcem.80.10.7559889

Eisenhofer, G., Timmers, H. J., Lenders, J. W., Bornstein, S. R., Tiebel, O., Mannelli, M., . . Pacak, K. (2011). Age at diagnosis of pheochromocytoma differs according to catecholamine phenotype and tumor location. J Clin Endocrinol Metab, 96(2), 375-384. doi:10.1210/jc.2010-1588

Ellis, L. M., \& Hicklin, D. J. (2008). VEGF-targeted therapy: mechanisms of anti-tumour activity. Nat Rev Cancer, 8(8), 579-591. doi:10.1038/nrc2403

Eng, C. (1999). RET proto-oncogene in the development of human cancer. J Clin Oncol, 17(1), 380-393.

Eng, C., \& Mulligan, L. M. (1997). Mutations of the RET proto-oncogene in the multiple endocrine neoplasia type 2 syndromes, related sporadic tumours, and hirschsprung disease. Hum Mutat, 9(2), 97-109. doi:10.1002/(SICI)1098-1004(1997)9:2<97::AID-HUMU1>3.0.CO;2-M [pii] 10.1002/(SICI)1098-1004(1997)9:2<97::AID-HUMU1>3.0.CO;2-M

Eng, C., Smith, D. P., Mulligan, L. M., Nagai, M. A., Healey, C. S., Ponder, M. A., . . e et al. (1994). Point mutation within the tyrosine kinase domain of the RET proto-oncogene in multiple endocrine neoplasia type 2B and related sporadic tumours. Hum Mol Genet, 3(2), 237-241. 
Enomoto, H., Crawford, P. A., Gorodinsky, A., Heuckeroth, R. O., Johnson, E. M., Jr., \& Milbrandt, J. (2001). RET signaling is essential for migration, axonal growth and axon guidance of developing sympathetic neurons. Development, 128(20), 3963-3974.

Favier, J., Amar, L., \& Gimenez-Roqueplo, A. P. (2014). Paraganglioma and phaeochromocytoma: from genetics to personalized medicine. Nat Rev Endocrinol. doi:10.1038/nrendo.2014.188 nrendo.2014.188 [pii]

Feijoo-Cuaresma, M., Mendez, F., Maqueda, A., Esteban, M. A., Naranjo-Suarez, S., Castellanos, M. C., . . . Calzada, M. J. (2008). Inadequate activation of the GTPase RhoA contributes to the lack of fibronectin matrix assembly in von Hippel-Lindau protein-defective renal cancer cells. J Biol Chem, 283(36), 24982-24990. doi:10.1074/jbc.M709390200

Fernandez, M. C., Martin, A., Venara, M., Calcagno Mde, L., Sanso, G., Quintana, S., . . Pennisi, P. A. (2013). Overexpression of the insulin-like growth factor 1 receptor (IGF-1R) is associated with malignancy in familial pheochromocytomas and paragangliomas. Clin Endocrinol (Oxf), 79(5), 623-630. doi:10.1111/cen.12205

Fikri, A. S., Kroiss, A., Ahmad, A. Z., Zanariah, H., Lau, W. F., Uprimny, C., . . Virgolini, I. J. (2014). Localization and prediction of malignant potential in recurrent pheochromocytoma/paraganglioma (PCC/PGL) using 18F-FDG PET/CT. Acta Radiol, 55(5), 631640. doi:10.1177/0284185113504330

Fischer, T., Doll, C., Jacobs, S., Kolodziej, A., Stumm, R., \& Schulz, S. (2008). Reassessment of sst2 somatostatin receptor expression in human normal and neoplastic tissues using the novel rabbit monoclonal antibody UMB-1. J Clin Endocrinol Metab, 93(11), 4519-4524. doi:jc.2008-1063 [pii] 10.1210/jc.2008-1063

Fishbein, L., Khare, S., Wubbenhorst, B., DeSloover, D., D'Andrea, K., Merrill, S., . . Nathanson, K. L. (2015). Whole-exome sequencing identifies somatic ATRX mutations in pheochromocytomas and paragangliomas. Nat Commun, 6, 6140. doi:10.1038/ncomms7140

Fisher, R., Horswell, S., Rowan, A., Salm, M. P., de Bruin, E. C., Gulati, S., . . Swanton, C. (2014). Development of synchronous VHL syndrome tumors reveals contingencies and constraints to tumor evolution. Genome Biol, 15(8), 433. doi:10.1186/s13059-014-0433-z

Flicker, K., Ulz, P., Hoger, H., Zeitlhofer, P., Haas, O. A., Behmel, A., . . Speicher, M. R. (2012). Highresolution analysis of alterations in medullary thyroid carcinoma genomes. Int J Cancer, 131(2), E66-73. doi:10.1002/ijc.26494

Floris, C., Rassu, S., Boccone, L., Gasperini, D., Cao, A., \& Crisponi, L. (2008). Two patients with balanced translocations and autistic disorder: CSMD3 as a candidate gene for autism found in their common 8q23 breakpoint area. Eur J Hum Genet, 16(6), 696-704. doi:10.1038/ejhg.2008.7

Gaal, J., Burnichon, N., Korpershoek, E., Roncelin, I., Bertherat, J., Plouin, P. F., . . Dinjens, W. N. (2010). Isocitrate dehydrogenase mutations are rare in pheochromocytomas and paragangliomas. J Clin Endocrinol Metab, 95(3), 1274-1278. doi:10.1210/jc.2009-2170

Gao, B., Meng, F., Bian, W., Chen, J., Zhao, H., Ma, G., ... Xu, Z. (2006). Development and validation of pheochromocytoma of the adrenal gland scaled score for predicting malignant pheochromocytomas. Urology, 68(2), 282-286. doi:10.1016/j.urology.2006.02.019

Gerlinger, M., Horswell, S., Larkin, J., Rowan, A. J., Salm, M. P., Varela, I., . . Swanton, C. (2014). Genomic architecture and evolution of clear cell renal cell carcinomas defined by multiregion sequencing. Nat Genet, 46(3), 225-233. doi:10.1038/ng.2891

Gerlinger, M., Rowan, A. J., Horswell, S., Larkin, J., Endesfelder, D., Gronroos, E., . . S Swanton, C. (2012). Intratumor heterogeneity and branched evolution revealed by multiregion sequencing. $N \mathrm{Eng} / \mathrm{J}$ Med, 366(10), 883-892. doi:10.1056/NEJMoa1113205

Gherasim, C., Lofgren, M., \& Banerjee, R. (2013). Navigating the B(12) road: assimilation, delivery, and disorders of cobalamin. J Biol Chem, 288(19), 13186-13193. doi:10.1074/jbc.R113.458810 
Goldstein, R. E., O'Neill, J. A., Jr., Holcomb, G. W., 3rd, Morgan, W. M., 3rd, Neblett, W. W., 3rd, Oates, J. A., . . Scott, H. W., Jr. (1999). Clinical experience over 48 years with pheochromocytoma. Ann Surg, 229(6), 755-764; discussion 764-756.

Gonzalez, D., Fearfield, L., Nathan, P., Taniere, P., Wallace, A., Brown, E., . . Whittaker, S. (2013). BRAF mutation testing algorithm for vemurafenib treatment in melanoma: recommendations from an expert panel. Br J Dermatol, 168(4), 700-707. doi:10.1111/bjd.12248

Gordan, J. D., Bertout, J. A., Hu, C. J., Diehl, J. A., \& Simon, M. C. (2007). HIF-2alpha promotes hypoxic cell proliferation by enhancing c-myc transcriptional activity. Cancer Cell, 11(4), 335-347. doi:10.1016/j.ccr.2007.02.006

Gundem, G., Van Loo, P., Kremeyer, B., Alexandrov, L. B., Tubio, J. M., Papaemmanuil, E., ... Bova, G. S. (2015). The evolutionary history of lethal metastatic prostate cancer. Nature, 520(7547), 353357. doi:10.1038/nature14347

Guo, J. U., Su, Y., Zhong, C., Ming, G. L., \& Song, H. (2011). Hydroxylation of 5-methylcytosine by TET1 promotes active DNA demethylation in the adult brain. Cell, 145(3), 423-434. doi:10.1016/j.cell.2011.03.022

Haller, F., Moskalev, E. A., Faucz, F. R., Barthelmess, S., Wiemann, S., Bieg, M., . . A Agaimy, A. (2014). Aberrant DNA hypermethylation of SDHC: a novel mechanism of tumor development in Carney triad. Endocr Relat Cancer, 21(4), 567-577. doi:10.1530/ERC-14-0254

Hanahan, D., \& Weinberg, R. A. (2011). Hallmarks of cancer: the next generation. Cell, 144(5), 646-674. doi:S0092-8674(11)00127-9 [pii] 10.1016/j.cell.2011.02.013

Hao, H. X., Khalimonchuk, O., Schraders, M., Dephoure, N., Bayley, J. P., Kunst, H., . . Rutter, J. (2009). $\mathrm{SDH} 5$, a gene required for flavination of succinate dehydrogenase, is mutated in paraganglioma. Science, 325(5944), 1139-1142. doi:1175689 [pii] 10.1126/science.1175689

Hata, J., Haga, N., Ishibashi, K., Takahashi, N., Ogawa, S., Kataoka, M., . . Kojima, Y. (2014). Sunitinib for refractory malignant pheochromocytoma: two case reports. Int Urol Nephrol, 46(7), 1309-1312. doi:10.1007/s11255-014-0663-8

Heider, A., \& Alt, R. (2013). virtualArray: a R/bioconductor package to merge raw data from different microarray platforms. BMC Bioinformatics, 14, 75. doi:10.1186/1471-2105-14-75

Henry, J. P., Botton, D., Sagne, C., Isambert, M. F., Desnos, C., Blanchard, V., . . Gasnier, B. (1994). Biochemistry and molecular biology of the vesicular monoamine transporter from chromaffin granules. J Exp Biol, 196, 251-262.

Holohan, C., Van Schaeybroeck, S., Longley, D. B., \& Johnston, P. G. (2013). Cancer drug resistance: an evolving paradigm. Nat Rev Cancer, 13(10), 714-726. doi:10.1038/nrc3599

Hompes, D., \& Ruers, T. (2011). Review: incidence and clinical significance of Bevacizumab-related nonsurgical and surgical serious adverse events in metastatic colorectal cancer. Eur J Surg Oncol, 37(9), 737-746. doi:10.1016/j.ejso.2011.06.004

Hon, W. C., Wilson, M. I., Harlos, K., Claridge, T. D., Schofield, C. J., Pugh, C. W., ... Jones, E. Y. (2002). Structural basis for the recognition of hydroxyproline in HIF-1 alpha by pVHL. Nature, 417(6892), 975-978. doi:10.1038/nature00767 nature00767 [pii]

Huang, S. C., Koch, C. A., Vortmeyer, A. O., Pack, S. D., Lichtenauer, U. D., Mannan, P., .. Zhuang, Z. (2000). Duplication of the mutant RET allele in trisomy 10 or loss of the wild-type allele in multiple endocrine neoplasia type 2-associated pheochromocytomas. Cancer Res, 60(22), 62236226.

Imhof, A., Brunner, P., Marincek, N., Briel, M., Schindler, C., Rasch, H., ... Walter, M. A. (2011). Response, survival, and long-term toxicity after therapy with the radiolabeled somatostatin analogue [90Y-DOTA]-TOC in metastasized neuroendocrine cancers. J Clin Oncol, 29(17), 24162423. doi:10.1200/JCO.2010.33.7873 
Inda, M. M., Bonavia, R., Mukasa, A., Narita, Y., Sah, D. W., Vandenberg, S., . . Furnari, F. (2010). Tumor heterogeneity is an active process maintained by a mutant EGFR-induced cytokine circuit in glioblastoma. Genes Dev, 24(16), 1731-1745. doi:10.1101/gad.1890510

International Cancer Genome, C., Hudson, T. J., Anderson, W., Artez, A., Barker, A. D., Bell, C., ... Yang, H. (2010). International network of cancer genome projects. Nature, 464(7291), 993-998. doi:10.1038/nature08987

Jafri, M., \& Maher, E. R. (2012). The genetics of phaeochromocytoma: using clinical features to guide genetic testing. Eur J Endocrinol, 166(2), 151-158. doi:10.1530/EJE-11-0497

Jamal-Hanjani, M., Hackshaw, A., Ngai, Y., Shaw, J., Dive, C., Quezada, S., . . Swanton, C. (2014). Tracking genomic cancer evolution for precision medicine: the lung TRACERx study. PLoS Biol, 12(7), e1001906. doi:10.1371/journal.pbio.1001906

Janeway, K. A., Kim, S. Y., Lodish, M., Nose, V., Rustin, P., Gaal, J., . . Stratakis, C. A. (2011). Defects in succinate dehydrogenase in gastrointestinal stromal tumors lacking KIT and PDGFRA mutations. Proc Natl Acad Sci U S A, 108(1), 314-318. doi:1009199108 [pii] 10.1073/pnas.1009199108

Janssen, I., Blanchet, E. M., Adams, K., Chen, C. C., Millo, C. M., Herscovitch, P., . . Pacak, K. (2015). Superiority of [68Ga]-DOTATATE PET/CT to Other Functional Imaging Modalities in the Localization of SDHB-Associated Metastatic Pheochromocytoma and Paraganglioma. Clin Cancer Res, 21(17), 3888-3895. doi:10.1158/1078-0432.CCR-14-2751

Jarbo, C., Buckley, P. G., Piotrowski, A., Mantripragada, K. K., Benetkiewicz, M., Diaz de Stahl, T., ... Dumanski, J. P. (2006). Detailed assessment of chromosome 22 aberrations in sporadic pheochromocytoma using array-CGH. Int J Cancer, 118(5), 1159-1164. doi:10.1002/ijc.21385

Jezequel, P., Loussouarn, D., Guerin-Charbonnel, C., Campion, L., Vanier, A., Gouraud, W., . . Campone, M. (2015). Gene-expression molecular subtyping of triple-negative breast cancer tumours: importance of immune response. Breast Cancer Res, 17(1), 43. doi:10.1186/s13058-015-0550-y

Jiang, S., \& Dahia, P. L. (2011). Minireview: the busy road to pheochromocytomas and paragangliomas has a new member, TMEM127. Endocrinology, 152(6), 2133-2140. doi:en.2011-0052 [pii] 10.1210/en.2011-0052

Jiao, Y., Shi, C., Edil, B. H., de Wilde, R. F., Klimstra, D. S., Maitra, A., ... Papadopoulos, N. (2011). DAXX/ATRX, MEN1, and mTOR pathway genes are frequently altered in pancreatic neuroendocrine tumors. Science, 331(6021), 1199-1203. doi:science.1200609 [pii] 10.1126/science.1200609

Jimenez, C., Cabanillas, M. E., Santarpia, L., Jonasch, E., Kyle, K. L., Lano, E. A., . . W Waguespack, S. G. (2009). Use of the tyrosine kinase inhibitor sunitinib in a patient with von Hippel-Lindau disease: targeting angiogenic factors in pheochromocytoma and other von Hippel-Lindau disease-related tumors. J Clin Endocrinol Metab, 94(2), 386-391. doi:10.1210/jc.2008-1972

Jimenez, C., Rohren, E., Habra, M. A., Rich, T., Jimenez, P., Ayala-Ramirez, M., \& Baudin, E. (2013). Current and future treatments for malignant pheochromocytoma and sympathetic paraganglioma. Curr Oncol Rep, 15(4), 356-371. doi:10.1007/s11912-013-0320-x

Jing, J., Junutula, J. R., Wu, C., Burden, J., Matern, H., Peden, A. A., \& Prekeris, R. (2010). FIP1/RCP binding to Golgin-97 regulates retrograde transport from recycling endosomes to the trans-Golgi network. Mol Biol Cell, 21(17), 3041-3053. doi:10.1091/mbc.E10-04-0313

Joshua, A. M., Ezzat, S., Asa, S. L., Evans, A., Broom, R., Freeman, M., \& Knox, J. J. (2009). Rationale and evidence for sunitinib in the treatment of malignant paraganglioma/pheochromocytoma. $J$ Clin Endocrinol Metab, 94(1), 5-9. doi:10.1210/jc.2008-1836

Juhlin, C. C., Stenman, A., Haglund, F., Clark, V. E., Brown, T. C., Baranoski, J., . . Carling, T. (2015). Whole-exome sequencing defines the mutational landscape of pheochromocytoma and identifies KMT2D as a recurrently mutated gene. Genes Chromosomes Cancer. doi:10.1002/gcc.22267 
Kaelin, W. G. (2007). Von Hippel-Lindau disease. Annu Rev Pathol, 2, 145-173. doi:10.1146/annurev.pathol.2.010506.092049

Kakinuma, H., Inomata, H., \& Kitamura, N. (2005). Enhanced JNK activation by NESK without kinase activity upon caspase-mediated cleavage during apoptosis. Cell Signal, 17(11), 1439-1448. doi:10.1016/j.cellsig.2005.03.004

Kawamoto, Y., Takeda, K., Okuno, Y., Yamakawa, Y., Ito, Y., Taguchi, R., . . Nakashima, I. (2004). Identification of RET autophosphorylation sites by mass spectrometry. J Biol Chem, 279(14), 14213-14224. doi:10.1074/jbc.M312600200

Keiser, H. R., Goldstein, D. S., Wade, J. L., Douglas, F. L., \& Averbuch, S. D. (1985). Treatment of malignant pheochromocytoma with combination chemotherapy. Hypertension, 7(3 Pt 2), 118-24.

Keith, B., Johnson, R. S., \& Simon, M. C. (2012). HIF1alpha and HIF2alpha: sibling rivalry in hypoxic tumour growth and progression. Nat Rev Cancer, 12(1), 9-22. doi:10.1038/nrc3183 nrc3183 [pii]

Khosravi-Far, R., \& Der, C. J. (1994). The Ras signal transduction pathway. Cancer Metastasis Rev, 13(1), 67-89.

Kibel, A., Iliopoulos, O., DeCaprio, J. A., \& Kaelin, W. G., Jr. (1995). Binding of the von Hippel-Lindau tumor suppressor protein to Elongin B and C. Science, 269(5229), 1444-1446.

Kim, H., Zheng, S., Amini, S. S., Virk, S. M., Mikkelsen, T., Brat, D. J., . . Verhaak, R. G. (2015). Wholegenome and multisector exome sequencing of primary and post-treatment glioblastoma reveals patterns of tumor evolution. Genome Res. doi:10.1101/gr.180612.114

Kim, T. M., Jung, S. H., An, C. H., Lee, S. H., Baek, I. P., Kim, M., . . Chung, Y. J. (2015). Subclonal genomic architectures of primary and metastatic colorectal cancer based on intratumoral genetic heterogeneity. Clin Cancer Res. doi:10.1158/1078-0432.CCR-14-2413

Kim, T. M., Jung, S. H., Baek, I. P., Lee, S. H., Choi, Y. J., Lee, J. Y., . . Lee, S. H. (2014). Regional biases in mutation screening due to intratumoural heterogeneity of prostate cancer. J Pathol, 233(4), 425-435. doi:10.1002/path.4380

King, A., Selak, M. A., \& Gottlieb, E. (2006). Succinate dehydrogenase and fumarate hydratase: linking mitochondrial dysfunction and cancer. Oncogene, 25(34), 4675-4682. doi:1209594 [pii] 10.1038/sj.onc.1209594

Klein, R. D., Salih, S., Bessoni, J., \& Bale, A. E. (2005). Clinical testing for multiple endocrine neoplasia type 1 in a DNA diagnostic laboratory. Genet Med, 7(2), 131-138. doi:00125817-20050200000007 [pii]

Knowles, P. P., Murray-Rust, J., Kjaer, S., Scott, R. P., Hanrahan, S., Santoro, M., . . McDonald, N. Q. (2006). Structure and chemical inhibition of the RET tyrosine kinase domain. J Biol Chem, 281(44), 33577-33587. doi:10.1074/jbc.M605604200

Knudson, A. G., Jr. (1971). Mutation and cancer: statistical study of retinoblastoma. Proc Natl Acad Sci U $S A, 68(4), 820-823$.

Ko, J. M., Sohn, Y. B., Jeong, S. Y., Kim, H. J., \& Messiaen, L. M. (2013). Mutation spectrum of NF1 and clinical characteristics in 78 Korean patients with neurofibromatosis type 1. Pediatr Neurol, 48(6), 447-453. doi:10.1016/j.pediatrneurol.2013.02.004 S0887-8994(13)00093-3 [pii]

Koboldt, D. C., Chen, K., Wylie, T., Larson, D. E., McLellan, M. D., Mardis, E. R., . . Ding, L. (2009). VarScan: variant detection in massively parallel sequencing of individual and pooled samples. Bioinformatics, 25(17), 2283-2285. doi:10.1093/bioinformatics/btp373

Koch, C. A., Huang, S. C., Moley, J. F., Azumi, N., Chrousos, G. P., Gagel, R. F., . . Vortmeyer, A. O. (2001). Allelic imbalance of the mutant and wild-type RET allele in MEN 2A-associated medullary thyroid carcinoma. Oncogene, 20(53), 7809-7811. doi:10.1038/sj.onc.1204991

Korf, B. R. (2000). Malignancy in neurofibromatosis type 1. Oncologist, 5(6), 477-485.

Korpershoek, E., Favier, J., Gaal, J., Burnichon, N., van Gessel, B., Oudijk, L., . . de Krijger, R. R. (2011). SDHA immunohistochemistry detects germline SDHA gene mutations in apparently sporadic 
paragangliomas and pheochromocytomas. J Clin Endocrinol Metab, 96(9), E1472-1476. doi:10.1210/jc.2011-1043

Korpershoek, E., Stobbe, C. K., van Nederveen, F. H., de Krijger, R. R., \& Dinjens, W. N. (2010). Intratumoral molecular heterogeneity in benign and malignant pheochromocytomas and extraadrenal sympathetic paragangliomas. Endocr Relat Cancer, 17(3), 653-662. doi:10.1677/ERC-100072

Koshiji, M., Kageyama, Y., Pete, E. A., Horikawa, I., Barrett, J. C., \& Huang, L. E. (2004). HIF-1alpha induces cell cycle arrest by functionally counteracting Myc. EMBO J, 23(9), 1949-1956. doi:10.1038/sj.emboj.7600196

Koshiji, M., To, K. K., Hammer, S., Kumamoto, K., Harris, A. L., Modrich, P., \& Huang, L. E. (2005). HIF1alpha induces genetic instability by transcriptionally downregulating MutSalpha expression. Mol Cell, 17(6), 793-803. doi:10.1016/j.molcel.2005.02.015

Krauthammer, M., Kong, Y., Ha, B. H., Evans, P., Bacchiocchi, A., McCusker, J. P., ... Halaban, R. (2012). Exome sequencing identifies recurrent somatic RAC1 mutations in melanoma. Nat Genet, 44(9), 1006-1014. doi:10.1038/ng.2359

Kulshreshtha, R., Ferracin, M., Wojcik, S. E., Garzon, R., Alder, H., Agosto-Perez, F. J., . . Ivan, M. (2007). A microRNA signature of hypoxia. Mol Cell Biol, 27(5), 1859-1867. doi:10.1128/MCB.01395-06

Kurban, G., Duplan, E., Ramlal, N., Hudon, V., Sado, Y., Ninomiya, Y., \& Pause, A. (2008). Collagen matrix assembly is driven by the interaction of von Hippel-Lindau tumor suppressor protein with hydroxylated collagen IV alpha 2. Oncogene, 27(7), 1004-1012. doi:10.1038/sj.onc.1210709

Kwong, L. N., Weiss, K. R., Haigis, K. M., \& Dove, W. F. (2008). Atm is a negative regulator of intestinal neoplasia. Oncogene, 27(7), 1013-1018. doi:10.1038/sj.onc.1210708

Ladroue, C., Carcenac, R., Leporrier, M., Gad, S., Le Hello, C., Galateau-Salle, F., ... Gardie, B. (2008). PHD2 mutation and congenital erythrocytosis with paraganglioma. N Engl J Med, 359(25), 26852692. doi:359/25/2685 [pii] 10.1056/NEJMoa0806277

Larson, D. E., Harris, C. C., Chen, K., Koboldt, D. C., Abbott, T. E., Dooling, D. J., . . . Ding, L. (2012). SomaticSniper: identification of somatic point mutations in whole genome sequencing data. Bioinformatics, 28(3), 311-317. doi:10.1093/bioinformatics/btr665

Larsson, C., Skogseid, B., Oberg, K., Nakamura, Y., \& Nordenskjold, M. (1988). Multiple endocrine neoplasia type 1 gene maps to chromosome 11 and is lost in insulinoma. Nature, 332(6159), 8587. doi:10.1038/332085a0

Lawrence, M. S., Stojanov, P., Mermel, C. H., Robinson, J. T., Garraway, L. A., Golub, T. R., . . Getz, G. (2014). Discovery and saturation analysis of cancer genes across 21 tumour types. Nature, 505(7484), 495-501. doi:10.1038/nature12912

Laycock-van Spyk, S., Thomas, N., Cooper, D. N., \& Upadhyaya, M. (2011). Neurofibromatosis type 1associated tumours: their somatic mutational spectrum and pathogenesis. Hum Genomics, 5(6), 623-690. doi:C06321321MV0675R [pii]

Lee, S., Nakamura, E., Yang, H., Wei, W., Linggi, M. S., Sajan, M. P., .. S Schlisio, S. (2005). Neuronal apoptosis linked to EgIN3 prolyl hydroxylase and familial pheochromocytoma genes: developmental culling and cancer. Cancer Cell, 8(2), 155-167. doi:S1535-6108(05)00224-2 [pii] 10.1016/j.ccr.2005.06.015

Lehninger, A. L., Nelson, D. L., \& Cox, M. M. (2000). Lehninger principles of biochemistry (3rd ed.). New York: Worth Publishers.

Lehtonen, H. J. (2011). Hereditary leiomyomatosis and renal cell cancer: update on clinical and molecular characteristics. Fam Cancer, 10(2), 397-411. doi:10.1007/s10689-011-9428-z

Lenders, J. W., Eisenhofer, G., Mannelli, M., \& Pacak, K. (2005). Phaeochromocytoma. Lancet, 366(9486), 665-675. doi:10.1016/S0140-6736(05)67139-5 
Lenders, J. W., Pacak, K., Walther, M. M., Linehan, W. M., Mannelli, M., Friberg, P., . . Eisenhofer, G. (2002). Biochemical diagnosis of pheochromocytoma: which test is best? JAMA, 287(11), 14271434.

Letouze, E., Martinelli, C., Loriot, C., Burnichon, N., Abermil, N., Ottolenghi, C., . . Favier, J. (2013). SDH mutations establish a hypermethylator phenotype in paraganglioma. Cancer Cell, 23(6), 739752. doi:10.1016/j.ccr.2013.04.018 S1535-6108(13)00183-9 [pii]

Lewis, P. W., Elsaesser, S. J., Noh, K. M., Stadler, S. C., \& Allis, C. D. (2010). Daxx is an H3.3-specific histone chaperone and cooperates with ATRX in replication-independent chromatin assembly at telomeres. Proc Natl Acad Sci U S A, 107(32), 14075-14080. doi:10.1073/pnas.1008850107

Li, H., \& Durbin, R. (2009). Fast and accurate short read alignment with Burrows-Wheeler transform. Bioinformatics, 25(14), 1754-1760. doi:10.1093/bioinformatics/btp324

Li, L., Kim, E., Yuan, H., Inoki, K., Goraksha-Hicks, P., Schiesher, R. L., . . Guan, K. L. (2010). Regulation of mTORC1 by the Rab and Arf GTPases. J Biol Chem, 285(26), 19705-19709. doi:10.1074/jbc.C110.102483

Li, L. T., Jiang, G., Chen, Q., \& Zheng, J. N. (2015). Ki67 is a promising molecular target in the diagnosis of cancer (review). Mol Med Rep, 11(3), 1566-1572. doi:10.3892/mmr.2014.2914

Liu, P., Morrison, C., Wang, L., Xiong, D., Vedell, P., Cui, P., .. You, M. (2012). Identification of somatic mutations in non-small cell lung carcinomas using whole-exome sequencing. Carcinogenesis, 33(7), 1270-1276. doi:10.1093/carcin/bgs148

Liu, T., Brown, T. C., Juhlin, C. C., Andreasson, A., Wang, N., Backdahl, M., . . L Larsson, C. (2014). The activating TERT promoter mutation $\mathrm{C} 228 \mathrm{~T}$ is recurrent in subsets of adrenal tumors. Endocr Relat Cancer, 21(3), 427-434. doi:10.1530/ERC-14-0016

Liu, W., Shen, S. M., Zhao, X. Y., \& Chen, G. Q. (2012). Targeted genes and interacting proteins of hypoxia inducible factor-1. Int J Biochem Mol Biol, 3(2), 165-178.

Lock, J. G., Hammond, L. A., Houghton, F., Gleeson, P. A., \& Stow, J. L. (2005). E-cadherin transport from the trans-Golgi network in tubulovesicular carriers is selectively regulated by golgin-97. Traffic, 6(12), 1142-1156. doi:10.1111/j.1600-0854.2005.00349.x

Loh, K. C., Fitzgerald, P. A., Matthay, K. K., Yeo, P. P., \& Price, D. C. (1997). The treatment of malignant pheochromocytoma with iodine-131 metaiodobenzylguanidine (131I-MIBG): a comprehensive review of 116 reported patients. J Endocrinol Invest, 20(11), 648-658.

Lovejoy, C. A., Li, W., Reisenweber, S., Thongthip, S., Bruno, J., de Lange, T., . . Consortium, A. L. T. S. C. (2012). Loss of ATRX, genome instability, and an altered DNA damage response are hallmarks of the alternative lengthening of telomeres pathway. PLoS Genet, 8(7), e1002772. doi:10.1371/journal.pgen.1002772

Luchetti, A., Walsh, D., Rodger, F., Clark, G., Martin, T., Irving, R., ... Maher, E. R. (2015). Profiling of somatic mutations in phaeochromocytoma and paraganglioma by targeted next generation sequencing analysis. Int J Endocrinol, 2015, 138573. doi:10.1155/2015/138573

Ma, L., Chen, Z., Erdjument-Bromage, H., Tempst, P., \& Pandolfi, P. P. (2005). Phosphorylation and functional inactivation of TSC2 by Erk implications for tuberous sclerosis and cancer pathogenesis. Cell, 121(2), 179-193. doi:10.1016/j.cell.2005.02.031

Maher, E. R. (2004). Von Hippel-Lindau disease. Curr Mol Med, 4(8), 833-842.

Margetts, C. D., Astuti, D., Gentle, D. C., Cooper, W. N., Cascon, A., Catchpoole, D., . . Maher, E. R. (2005). Epigenetic analysis of HIC1, CASP8, FLIP, TSP1, DCR1, DCR2, DR4, DR5, KvDMR1, H19 and preferential 11p15.5 maternal-allele loss in von Hippel-Lindau and sporadic phaeochromocytomas. Endocr Relat Cancer, 12(1), 161-172. doi:10.1677/erc.1.00865

Maris, J. M., \& Knudson, A. G. (2015). Revisiting tissue specificity of germline cancer predisposing mutations. Nat Rev Cancer, 15(2), 65-66. 
Marsh, D. J., Theodosopoulos, G., Martin-Schulte, K., Richardson, A. L., Philips, J., Roher, H. D., . . . Robinson, B. G. (2003). Genome-wide copy number imbalances identified in familial and sporadic medullary thyroid carcinoma. J Clin Endocrinol Metab, 88(4), 1866-1872. doi:10.1210/jc.2002-021155

Martucci, V. L., \& Pacak, K. (2014). Pheochromocytoma and paraganglioma: diagnosis, genetics, management, and treatment. Curr Probl Cancer, 38(1), 7-41. doi:10.1016/j.currproblcancer.2014.01.001

Maxwell, P. H., Pugh, C. W., \& Ratcliffe, P. J. (2001). Activation of the HIF pathway in cancer. Curr Opin Genet Dev, 11(3), 293-299.

Maxwell, P. H., Wiesener, M. S., Chang, G. W., Clifford, S. C., Vaux, E. C., Cockman, M. E., ... Ratcliffe, P. J. (1999). The tumour suppressor protein VHL targets hypoxia-inducible factors for oxygendependent proteolysis. Nature, 399(6733), 271-275. doi:10.1038/20459

Mazumdar, J., Hickey, M. M., Pant, D. K., Durham, A. C., Sweet-Cordero, A., Vachani, A., ... Keith, B. (2010). HIF-2alpha deletion promotes Kras-driven lung tumor development. Proc Natl Acad Sci U S A, 107(32), 14182-14187. doi:10.1073/pnas.1001296107

Mazza, A., Armigliato, M., Marzola, M. C., Schiavon, L., Montemurro, D., Vescovo, G., . . Rubello, D. (2014). Anti-hypertensive treatment in pheochromocytoma and paraganglioma: current management and therapeutic features. Endocrine, 45(3), 469-478. doi:10.1007/s12020-013$0007-y$

McGranahan, N., \& Swanton, C. (2015). Biological and Therapeutic Impact of Intratumor Heterogeneity in Cancer Evolution. Cancer Cell, 27(1), 15-26. doi:10.1016/j.ccell.2014.12.001

McKenna, A., Hanna, M., Banks, E., Sivachenko, A., Cibulskis, K., Kernytsky, A., . . DePristo, M. A. (2010). The Genome Analysis Toolkit: a MapReduce framework for analyzing next-generation DNA sequencing data. Genome Res, 20(9), 1297-1303. doi:10.1101/gr.107524.110

McPherson, A., Hormozdiari, F., Zayed, A., Giuliany, R., Ha, G., Sun, M. G., . . Shah, S. P. (2011). deFuse: an algorithm for gene fusion discovery in tumor RNA-Seq data. PLoS Comput Biol, 7(5), e1001138. doi:10.1371/journal.pcbi.1001138 10-PLCB-RA-2589R4 [pii]

Meldrum, C., Doyle, M. A., \& Tothill, R. W. (2011). Next-generation sequencing for cancer diagnostics: a practical perspective. Clin Biochem Rev, 32(4), 177-195.

Melillo, R. M., Barone, M. V., Lupoli, G., Cirafici, A. M., Carlomagno, F., Visconti, R., .. Santoro, M. (1999). Ret-mediated mitogenesis requires Src kinase activity. Cancer Res, 59(5), 1120-1126.

Melillo, R. M., Santoro, M., Ong, S. H., Billaud, M., Fusco, A., Hadari, Y. R., . . Lax, I. (2001). Docking protein FRS2 links the protein tyrosine kinase RET and its oncogenic forms with the mitogenactivated protein kinase signaling cascade. Mol Cell Biol, 21(13), 4177-4187. doi:10.1128/MCB.21.13.4177-4187.2001

Metz, M., Gassmann, M., Fakler, B., Schaeren-Wiemers, N., \& Bettler, B. (2011). Distribution of the auxiliary GABAB receptor subunits KCTD8, 12, 12b, and 16 in the mouse brain. J Comp Neurol, 519(8), 1435-1454. doi:10.1002/cne.22610

Meyer-Rochow, G. Y., Jackson, N. E., Conaglen, J. V., Whittle, D. E., Kunnimalaiyaan, M., Chen, H., .. . Sidhu, S. B. (2010). MicroRNA profiling of benign and malignant pheochromocytomas identifies novel diagnostic and therapeutic targets. Endocr Relat Cancer, 17(3), 835-846. doi:10.1677/ERC10-0142

Mills, K. I., Kohlmann, A., Williams, P. M., Wieczorek, L., Liu, W. M., Li, R., ... Haferlach, T. (2009). Microarray-based classifiers and prognosis models identify subgroups with distinct clinical outcomes and high risk of AML transformation of myelodysplastic syndrome. Blood, 114(5), 1063-1072. doi:10.1182/blood-2008-10-187203 
Mittal, D., Gubin, M. M., Schreiber, R. D., \& Smyth, M. J. (2014). New insights into cancer immunoediting and its three component phases--elimination, equilibrium and escape. Curr Opin Immunol, 27, 16-25. doi:10.1016/j.coi.2014.01.004

Mole, D. R., Blancher, C., Copley, R. R., Pollard, P. J., Gleadle, J. M., Ragoussis, J., \& Ratcliffe, P. J. (2009). Genome-wide association of hypoxia-inducible factor (HIF)-1alpha and HIF-2alpha DNA binding with expression profiling of hypoxia-inducible transcripts. J Biol Chem, 284(25), 16767-16775. doi:10.1074/jbc.M901790200

Mroz, E. A., \& Rocco, J. W. (2013). MATH, a novel measure of intratumor genetic heterogeneity, is high in poor-outcome classes of head and neck squamous cell carcinoma. Oral Oncol, 49(3), 211-215. doi:10.1016/j.oraloncology.2012.09.007

Mulligan, L. M., Eng, C., Healey, C. S., Clayton, D., Kwok, J. B., Gardner, E., . . . et al. (1994). Specific mutations of the RET proto-oncogene are related to disease phenotype in MEN 2A and FMTC. Nat Genet, 6(1), 70-74. doi:10.1038/ng0194-70

Munirajan, A. K., Ando, K., Mukai, A., Takahashi, M., Suenaga, Y., Ohira, M., . . Nakagawara, A. (2008). KIF1Bbeta functions as a haploinsufficient tumor suppressor gene mapped to chromosome 1 p36.2 by inducing apoptotic cell death. J Biol Chem, 283(36), 24426-24434. doi:10.1074/jbc.M802316200

Murakumo, Y., Jijiwa, M., Asai, N., Ichihara, M., \& Takahashi, M. (2006). RET and neuroendocrine tumors. Pituitary, 9(3), 179-192. doi:10.1007/s11102-006-0263-4

Murtaza, M., Dawson, S. J., Tsui, D. W., Gale, D., Forshew, T., Piskorz, A. M., . . Rosenfeld, N. (2013). Non-invasive analysis of acquired resistance to cancer therapy by sequencing of plasma DNA. Nature. doi:10.1038/nature12065 nature12065 [pii]

Nik-Zainal, S., Van Loo, P., Wedge, D. C., Alexandrov, L. B., Greenman, C. D., Lau, K. W., ... Campbell, P. J. (2012). The life history of 21 breast cancers. Cell, 149(5), 994-1007. doi:S0092-8674(12)005272 [pii] 10.1016/j.cell.2012.04.023

Nolting, S., \& Grossman, A. B. (2012). Signaling pathways in pheochromocytomas and paragangliomas: prospects for future therapies. Endocr Pathol, 23(1), 21-33. doi:10.1007/s12022-012-9199-6

Nowell, P. C. (1976). The clonal evolution of tumor cell populations. Science, 194(4260), 23-28.

Oberg, K. (2005). Somatostatin-receptor mediated diagnosis and treatment in gastrointestinal neuroendocrine tumours (GEP-NET's). Rocz Akad Med Bialymst, 50, 62-68.

Ohh, M., Yauch, R. L., Lonergan, K. M., Whaley, J. M., Stemmer-Rachamimov, A. O., Louis, D. N., ... Iliopoulos, O. (1998). The von Hippel-Lindau tumor suppressor protein is required for proper assembly of an extracellular fibronectin matrix. Mol Cell, 1(7), 959-968.

Opocher, G., \& Schiavi, F. (2011). Functional consequences of succinate dehydrogenase mutations. Endocr Pract, 17 Supp/ 3, 64-71. doi:L8634060N1M24622 [pii] 10.4158/EP11070.RA

Oudijk, L., de Krijger, R. R., Rapa, I., Beuschlein, F., de Cubas, A. A., Dei Tos, A. P., . . Volante, M. (2014). $\mathrm{H}$-RAS mutations are restricted to sporadic pheochromocytomas lacking specific clinical or pathological features: data from a multi-institutional series. J Clin Endocrinol Metab, 99(7), E1376-1380. doi:10.1210/jc.2013-3879

Parenti, G., Zampetti, B., Rapizzi, E., Ercolino, T., Giache, V., \& Mannelli, M. (2012). Updated and new perspectives on diagnosis, prognosis, and therapy of malignant pheochromocytoma/paraganglioma. J Oncol, 2012, 872713. doi:10.1155/2012/872713

Park, J., Song, C., Park, M., Yoo, S., Park, S. J., Hong, S., . . Ahn, H. (2011). Predictive characteristics of malignant pheochromocytoma. Korean J Urol, 52(4), 241-246. doi:10.4111/kju.2011.52.4.241

Pasini, B., McWhinney, S. R., Bei, T., Matyakhina, L., Stergiopoulos, S., Muchow, M., . . Stratakis, C. A. (2008). Clinical and molecular genetics of patients with the Carney-Stratakis syndrome and germline mutations of the genes coding for the succinate dehydrogenase subunits SDHB, SDHC, and SDHD. Eur J Hum Genet, 16(1), 79-88. doi:5201904 [pii] 10.1038/sj.ejhg.5201904 
Patterson, E., Webb, R., Weisbrod, A., Bian, B., He, M., Zhang, L., ... Kebebew, E. (2012). The microRNA expression changes associated with malignancy and SDHB mutation in pheochromocytoma. Endocr Relat Cancer, 19(2), 157-166. doi:10.1530/ERC-11-0308

Peng, L., Yang, Z., Tan, C., Ren, G., \& Chen, J. (2013). Epigenetic inactivation of ADAMTS9 via promoter methylation in multiple myeloma. Mol Med Rep, 7(3), 1055-1061. doi:10.3892/mmr.2013.1291

Peng, Z. G., Zhou, M. Y., Huang, Y., Qiu, J. H., Wang, L. S., Liao, S. H., . . Chen, G. Q. (2008). Physical and functional interaction of Runt-related protein 1 with hypoxia-inducible factor-1alpha. Oncogene, 27(6), 839-847. doi:10.1038/sj.onc.1210676

Peterlongo, P., Chang-Claude, J., Moysich, K. B., Rudolph, A., Schmutzler, R. K., Simard, J., . . Friedman, E. (2015). Candidate genetic modifiers for breast and ovarian cancer risk in BRCA1 and BRCA2 mutation carriers. Cancer Epidemiol Biomarkers Prev, 24(1), 308-316. doi:10.1158/10559965.EPI-14-0532

Petri, B. J., Speel, E. J., Korpershoek, E., Claessen, S. M., van Nederveen, F. H., Giesen, V., . . de Krijger, R. R. (2008). Frequent loss of $17 \mathrm{p}$, but no $\mathrm{p} 53$ mutations or protein overexpression in benign and malignant pheochromocytomas. Mod Pathol, 21(4), 407-413. doi:3801013 [pii] 10.1038/modpathol.3801013

Plaza Menacho, I., Koster, R., van der Sloot, A. M., Quax, W. J., Osinga, J., van der Sluis, T., ... Hofstra, R. M. (2005). RET-familial medullary thyroid carcinoma mutants Y791F and S891A activate a $\mathrm{Src/JAK/STAT3}$ pathway, independent of glial cell line-derived neurotrophic factor. Cancer Res, 65(5), 1729-1737. doi:10.1158/0008-5472.CAN-04-2363

Prazeres, H., Torres, J., Rodrigues, F., Couto, J. P., Vinagre, J., Sobrinho-Simoes, M., \& Soares, P. (2011). How to Treat a Signal? Current Basis for RET-Genotype-Oriented Choice of Kinase Inhibitors for the Treatment of Medullary Thyroid Cancer. J Thyroid Res, 2011, 678357. doi:10.4061/2011/678357

Pylayeva-Gupta, Y., Grabocka, E., \& Bar-Sagi, D. (2011). RAS oncogenes: weaving a tumorigenic web. Nat Rev Cancer, 11(11), 761-774. doi:10.1038/nrc3106

Qi, Y., Su, T., Zhang, X., Jiang, Y., Yuan, W., Wang, W., . . Ning, G. (2012). Gene expression profiles of phaeochromocytomas with ERBB2 overexpression reveal a new molecular mechanism tumourigenicity. Clin Endocrinol (Oxf), 77(3), 399-406. doi:10.1111/j.1365-2265.2012.04388.x

Qin, N., de Cubas, A. A., Garcia-Martin, R., Richter, S., Peitzsch, M., Menschikowski, M., . . Eisenhofer, G. (2014). Opposing effects of HIF1alpha and HIF2alpha on chromaffin cell phenotypic features and tumor cell proliferation: Insights from MYC-associated factor X. Int J Cancer, 135(9), 20542064. doi:10.1002/ijc.28868

Qin, Y., Deng, Y., Ricketts, C. J., Srikantan, S., Wang, E., Maher, E. R., \& Dahia, P. L. (2014). The tumor susceptibility gene TMEM127 is mutated in renal cell carcinomas and modulates endolysosomal function. Hum Mol Genet, 23(9), 2428-2439. doi:10.1093/hmg/ddt638

Qin, Y., Yao, L., King, E. E., Buddavarapu, K., Lenci, R. E., Chocron, E. S., . . Dahia, P. L. (2010). Germline mutations in TMEM127 confer susceptibility to pheochromocytoma. Nat Genet, 42(3), 229-233. doi:ng.533 [pii] 10.1038/ng.533

Rahman, N. (2014). Realizing the promise of cancer predisposition genes. Nature, 505(7483), 302-308. doi:10.1038/nature12981

Rakoff-Nahoum, S. (2006). Why cancer and inflammation? Yale J Biol Med, 79(3-4), 123-130.

Rapa, I., Saggiorato, E., Giachino, D., Palestini, N., Orlandi, F., Papotti, M., \& Volante, M. (2011). Mammalian target of rapamycin pathway activation is associated to RET mutation status in medullary thyroid carcinoma. J Clin Endocrinol Metab, 96(7), 2146-2153. doi:10.1210/jc.20102655 
Ricketts, C. J., Forman, J. R., Rattenberry, E., Bradshaw, N., Lalloo, F., Izatt, L., . . Maher, E. R. (2010). Tumor risks and genotype-phenotype-proteotype analysis in 358 patients with germline mutations in SDHB and SDHD. Hum Mutat, 31(1), 41-51. doi:10.1002/humu.21136

Rinn, J. L., \& Chang, H. Y. (2012). Genome regulation by long noncoding RNAs. Annu Rev Biochem, 81, 145-166. doi:10.1146/annurev-biochem-051410-092902

Robinson, M. D., McCarthy, D. J., \& Smyth, G. K. (2010). edgeR: a Bioconductor package for differential expression analysis of digital gene expression data. Bioinformatics, 26(1), 139-140. doi:10.1093/bioinformatics/btp616

Romero, O. A., Torres-Diz, M., Pros, E., Savola, S., Gomez, A., Moran, S., . . Sanchez-Cespedes, M. (2014). MAX inactivation in small cell lung cancer disrupts MYC-SWI/SNF programs and is synthetic lethal with BRG1. Cancer Discov, 4(3), 292-303. doi:10.1158/2159-8290.CD-13-0799

Roth, A., Ding, J., Morin, R., Crisan, A., Ha, G., Giuliany, R., . . Shah, S. P. (2012). JointSNVMix: a probabilistic model for accurate detection of somatic mutations in normal/tumour paired nextgeneration sequencing data. Bioinformatics, 28(7), 907-913. doi:10.1093/bioinformatics/bts053

Rozen, E. J., Schmidt, H., Dolcet, X., Basson, M. A., Jain, S., \& Encinas, M. (2009). Loss of Sprouty1 rescues renal agenesis caused by Ret mutation. J Am Soc Nephrol, 20(2), 255-259. doi:ASN.2008030267 [pii] 10.1681/ASN.2008030267

Rufini, V., Calcagni, M. L., \& Baum, R. P. (2006). Imaging of neuroendocrine tumors. Semin Nucl Med, 36(3), 228-247. doi:S0001-2998(06)00018-3 [pii] 10.1053/j.semnuclmed.2006.03.007

Russell, N., Delatycki, M., \& Grossmann, M. (2015). Metastatic phaeochromocytoma in a 23-year-old woman with an unclassified variant in the von Hippel Lindau disease gene: how can the pathogenicity of this variant be determined? Clin Endocrinol (Oxf), 83(1), 15-19. doi:10.1111/cen.12710

Russell, R. C., \& Ohh, M. (2008). NEDD8 acts as a 'molecular switch' defining the functional selectivity of VHL. EMBO Rep, 9(5), 486-491. doi:10.1038/embor.2008.19 embor200819 [pii]

Sakamoto, N., Shibuya, K., Shimizu, Y., Yotsumoto, K., Miyabayashi, T., Sakano, S., . . Shibuya, A. (2001). A novel Fc receptor for IgA and IgM is expressed on both hematopoietic and non-hematopoietic tissues. Eur J Immunol, 31(5), 1310-1316. doi:10.1002/1521-4141(200105)31:5<1310::AIDIMMU1310>3.0.CO;2-N

Sandgren, J., Andersson, R., Rada-Iglesias, A., Enroth, S., Akerstrom, G., Dumanski, J. P., . . W Wadelius, C. (2010). Integrative epigenomic and genomic analysis of malignant pheochromocytoma. Exp Mol Med, 42(7), 484-502. doi:emm.2010.42.050 [pii] 10.3858/emm.2010.42.7.050

Sandgren, J., Diaz de Stahl, T., Andersson, R., Menzel, U., Piotrowski, A., Nord, H., . . Westin, G. (2010). Recurrent genomic alterations in benign and malignant pheochromocytomas and paragangliomas revealed by whole-genome array comparative genomic hybridization analysis. Endocr Relat Cancer, 17(3), 561-579. doi:10.1677/ERC-09-0310 ERC-09-0310 [pii]

Santoro, M., Carlomagno, F., Romano, A., Bottaro, D. P., Dathan, N. A., Grieco, M., . . et al. (1995). Activation of RET as a dominant transforming gene by germline mutations of MEN2A and MEN2B. Science, 267(5196), 381-383.

Sato, H. (1950). On the chromosomes of the malignant tumor (studies on Yoshida sarcoma). Gan, 41(24), 198-200.

Saxena, N., Lahiri, S. S., Hambarde, S., \& Tripathi, R. P. (2008). RAS: target for cancer therapy. Cancer Invest, 26(9), 948-955. doi:10.1080/07357900802087275

Schiavi, F., Boedeker, C. C., Bausch, B., Peczkowska, M., Gomez, C. F., Strassburg, T., . . Neumann, H. P. (2005). Predictors and prevalence of paraganglioma syndrome associated with mutations of the SDHC gene. JAMA, 294(16), 2057-2063. doi:294/16/2057 [pii] 10.1001/jama.294.16.2057

Schlisio, S., Kenchappa, R. S., Vredeveld, L. C., George, R. E., Stewart, R., Greulich, H., . . Kaelin, W. G., Jr. (2008). The kinesin KIF1Bbeta acts downstream from EgIN3 to induce apoptosis and is a 
potential 1p36 tumor suppressor. Genes Dev, 22(7), 884-893. doi:10.1101/gad.1648608 gad.1648608 [pii]

Schulz, S., Pauli, S. U., Handel, M., Dietzmann, K., Firsching, R., \& Hollt, V. (2000). Immunohistochemical determination of five somatostatin receptors in meningioma reveals frequent overexpression of somatostatin receptor subtype sst2A. Clin Cancer Res, 6(5), 1865-1874.

Schwarzenbach, H., Hoon, D. S., \& Pantel, K. (2011). Cell-free nucleic acids as biomarkers in cancer patients. Nat Rev Cancer, 11(6), 426-437. doi:10.1038/nrc3066

Sewastianik, T., Prochorec-Sobieszek, M., Chapuy, B., \& Juszczynski, P. (2014). MYC deregulation in lymphoid tumors: molecular mechanisms, clinical consequences and therapeutic implications. Biochim Biophys Acta, 1846(2), 457-467. doi:10.1016/j.bbcan.2014.08.006

Sexton, A., Rawlings, L., McKavanagh, G., Simons, K., \& Winship, I. (2015). A Novel von Hippel Lindau Gene Intronic Variant and Its Reclassification from VUS to Pathogenic: the Impact on a Large Family. J Genet Couns. doi:10.1007/s10897-015-9875-z

Shimizu, A., Asakawa, S., Sasaki, T., Yamazaki, S., Yamagata, H., Kudoh, J., . . Shimizu, N. (2003). A novel giant gene CSMD3 encoding a protein with CUB and sushi multiple domains: a candidate gene for benign adult familial myoclonic epilepsy on human chromosome 8q23.3-q24.1. Biochem Biophys Res Commun, 309(1), 143-154.

Smith, E. H., Janknecht, R., \& Maher, L. J., 3rd. (2007). Succinate inhibition of alpha-ketoglutaratedependent enzymes in a yeast model of paraganglioma. Hum Mol Genet, 16(24), 3136-3148. doi:10.1093/hmg/ddm275

Smyth, G. (2005). limma: Linear Models for Microarray Data. In R. Gentleman, V. J. Carey, W. Huber, R. A. Irizarry, \& S. Dudoit (Eds.), Bioinformatics and Computational Biology Solutions Using $R$ and Bioconductor (pp. 397-420). New York: Springer New York.

Stebbins, C. E., Kaelin, W. G., Jr., \& Pavletich, N. P. (1999). Structure of the VHL-ElonginC-ElonginB complex: implications for VHL tumor suppressor function. Science, 284(5413), 455-461.

Stenstrom, G., \& Svardsudd, K. (1986). Pheochromocytoma in Sweden 1958-1981. An analysis of the National Cancer Registry Data. Acta Med Scand, 220(3), 225-232.

Suh, I., Shibru, D., Eisenhofer, G., Pacak, K., Duh, Q. Y., Clark, O. H., \& Kebebew, E. (2009). Candidate genes associated with malignant pheochromocytomas by genome-wide expression profiling. Ann Surg, 250(6), 983-990. doi:10.1097/SLA.0b013e3181b248bb

Supek, F., Minana, B., Valcarcel, J., Gabaldon, T., \& Lehner, B. (2014). Synonymous mutations frequently act as driver mutations in human cancers. Cell, 156(6), 1324-1335. doi:10.1016/j.cell.2014.01.051

Tada, K., Okuda, Y., \& Yamashita, K. (1998). Three cases of malignant pheochromocytoma treated with cyclophosphamide, vincristine, and dacarbazine combination chemotherapy and alpha-methylp-tyrosine to control hypercatecholaminemia. Horm Res, 49(6), 295-297.

Takiyyuddin, M. A., Brown, M. R., Dinh, T. Q., Cervenka, J. H., Braun, S. D., Parmer, R. J., .. . O'Connor, D. T. (1994). Sympatho-adrenal secretion in humans: factors governing catecholamine and storage vesicle peptide co-release. J Auton Pharmacol, 14(3), 187-200.

Theos, A., \& Korf, B. R. (2006). Pathophysiology of neurofibromatosis type 1. Ann Intern Med, 144(11), 842-849. doi:144/11/842 [pii]

Thompson, L. D. (2002). Pheochromocytoma of the Adrenal gland Scaled Score (PASS) to separate benign from malignant neoplasms: a clinicopathologic and immunophenotypic study of 100 cases. Am J Surg Pathol, 26(5), 551-566.

Timmers, H. J., Kozupa, A., Chen, C. C., Carrasquillo, J. A., Ling, A., Eisenhofer, G., . . Pacak, K. (2007). Superiority of fluorodeoxyglucose positron emission tomography to other functional imaging techniques in the evaluation of metastatic SDHB-associated pheochromocytoma and paraganglioma. J Clin Oncol, 25(16), 2262-2269. doi:10.1200/JCO.2006.09.6297 
Toledo, R. A., \& Dahia, P. L. (2015). Next-generation sequencing for the diagnosis of hereditary pheochromocytoma and paraganglioma syndromes. Curr Opin Endocrinol Diabetes Obes, 22(3), 169-179. doi:10.1097/MED.0000000000000150

Toledo, R. A., Qin, Y., Srikantan, S., Morales, N. P., Li, Q., Deng, Y., . . Dahia, P. (2013). In vivo and in vitro oncogenic effects of HIF2A mutations in pheochromocytomas and paragangliomas. Endocr Relat Cancer. doi:ERC-13-0101 [pii] 10.1530/ERC-13-0101

Tothill, R. W., Tinker, A. V., George, J., Brown, R., Fox, S. B., Lade, S., . . Bowtell, D. D. (2008). Novel molecular subtypes of serous and endometrioid ovarian cancer linked to clinical outcome. Clin Cancer Res, 14(16), 5198-5208. doi:10.1158/1078-0432.CCR-08-0196

Trapnell, C., Pachter, L., \& Salzberg, S. L. (2009). TopHat: discovering splice junctions with RNA-Seq. Bioinformatics, 25(9), 1105-1111. doi:10.1093/bioinformatics/btp120

Trump, D., Farren, B., Wooding, C., Pang, J. T., Besser, G. M., Buchanan, K. D., . . Thakker, R. V. (1996). Clinical studies of multiple endocrine neoplasia type 1 (MEN1). QJM, 89(9), 653-669.

Turner, N. C., \& Reis-Filho, J. S. (2012). Genetic heterogeneity and cancer drug resistance. Lancet Oncol, 13(4), e178-185. doi:10.1016/S1470-2045(11)70335-7

van Essen, M., Krenning, E. P., Kam, B. L., de Jong, M., Valkema, R., \& Kwekkeboom, D. J. (2009). Peptide-receptor radionuclide therapy for endocrine tumors. Nat Rev Endocrinol, 5(7), 382-393. doi:nrendo.2009.105 [pii] 10.1038/nrendo.2009.105

van Essen, M., Krenning, E. P., Kooij, P. P., Bakker, W. H., Feelders, R. A., de Herder, W. W., ... Kwekkeboom, D. J. (2006). Effects of therapy with [177Lu-DOTA0, Tyr3]octreotate in patients with paraganglioma, meningioma, small cell lung carcinoma, and melanoma. J Nucl Med, 47(10), 1599-1606.

van Hulsteijn, L. T., Niemeijer, N. D., Dekkers, O. M., \& Corssmit, E. P. (2014). (131)I-MIBG therapy for malignant paraganglioma and phaeochromocytoma: systematic review and meta-analysis. Clin Endocrinol (Oxf), 80(4), 487-501. doi:10.1111/cen.12341

Van Loo, P., Nordgard, S. H., Lingjaerde, O. C., Russnes, H. G., Rye, I. H., Sun, W., ... Kristensen, V. N. (2010). Allele-specific copy number analysis of tumors. Proc Natl Acad Sci U S A, 107(39), 1691016915. doi:10.1073/pnas.1009843107

van Nederveen, F. H., Korpershoek, E., deLeeuw, R. J., Verhofstad, A. A., Lenders, J. W., Dinjens, W. N., . . . de Krijger, R. R. (2009). Array-comparative genomic hybridization in sporadic benign pheochromocytomas. Endocr Relat Cancer, 16(2), 505-513. doi:ERC-08-0241 [pii] 10.1677/ERC08-0241

Vanderveen, K. A., Thompson, S. M., Callstrom, M. R., Young, W. F., Jr., Grant, C. S., Farley, D. R., . . Thompson, G. B. (2009). Biopsy of pheochromocytomas and paragangliomas: potential for disaster. Surgery, 146(6), 1158-1166. doi:10.1016/j.surg.2009.09.013

VanderWeele, D. J., Brown, C. D., Taxy, J. B., Gillard, M., Hatcher, D. M., Tom, W. R., .. White, K. P. (2014). Low-grade prostate cancer diverges early from high grade and metastatic disease. Cancer Sci, 105(8), 1079-1085. doi:10.1111/cas.12460

Varier, R. A., \& Timmers, H. T. (2011). Histone lysine methylation and demethylation pathways in cancer. Biochim Biophys Acta, 1815(1), 75-89. doi:10.1016/j.bbcan.2010.10.002

Vogelstein, B., Papadopoulos, N., Velculescu, V. E., Zhou, S., Diaz, L. A., Jr., \& Kinzler, K. W. (2013). Cancer genome landscapes. Science, 339(6127), 1546-1558. doi:10.1126/science.1235122 339/6127/1546 [pii]

Wafelman, A. R., Hoefnagel, C. A., Maes, R. A., \& Beijnen, J. H. (1994). Radioiodinated metaiodobenzylguanidine: a review of its biodistribution and pharmacokinetics, drug interactions, cytotoxicity and dosimetry. Eur J Nucl Med, 21(6), 545-559. 
Waldmann, J., Langer, P., Habbe, N., Fendrich, V., Ramaswamy, A., Rothmund, M., . . Slater, E. P. (2009). Mutations and polymorphisms in the SDHB, SDHD, VHL, and RET genes in sporadic and familial pheochromocytomas. Endocrine, 35(3), 347-355. doi:10.1007/s12020-009-9178-y

Welander, J., Larsson, C., Backdahl, M., Hareni, N., Sivler, T., Brauckhoff, M., . . Gimm, O. (2012). Integrative genomics reveals frequent somatic NF1 mutations in sporadic pheochromocytomas. Hum Mol Genet. doi:dds402 [pii] 10.1093/hmg/dds402

Westermark, U. K., Wilhelm, M., Frenzel, A., \& Henriksson, M. A. (2011). The MYCN oncogene and differentiation in neuroblastoma. Semin Cancer Biol, 21(4), 256-266. doi:10.1016/j.semcancer.2011.08.001 S1044-579X(11)00049-6 [pii]

Wilkerson, M., \& Waltman, P. (2013). ConsensusClusterPlus: ConsensusCluster-Plus. R package (Version 1.20.0).

Wu, D., Tischler, A. S., Lloyd, R. V., DeLellis, R. A., de Krijger, R., van Nederveen, F., \& Nose, V. (2009). Observer variation in the application of the Pheochromocytoma of the Adrenal Gland Scaled Score. Am J Surg Pathol, 33(4), 599-608. doi:10.1097/PAS.0b013e318190d12e

Xia, X., Lemieux, M. E., Li, W., Carroll, J. S., Brown, M., Liu, X. S., \& Kung, A. L. (2009). Integrative analysis of HIF binding and transactivation reveals its role in maintaining histone methylation homeostasis. Proc Natl Acad Sci U S A, 106(11), 4260-4265. doi:10.1073/pnas.0810067106

Xiao, M., Yang, H., Xu, W., Ma, S., Lin, H., Zhu, H., . . Guan, K. L. (2012). Inhibition of alpha-KGdependent histone and DNA demethylases by fumarate and succinate that are accumulated in mutations of FH and SDH tumor suppressors. Genes Dev, 26(12), 1326-1338. doi:10.1101/gad.191056.112 gad.191056.112 [pii]

Xu, H., Zhu, X., Xu, Z., Hu, Y., Bo, S., Xing, T., \& Zhu, K. (2015). Non-invasive Analysis of Genomic Copy Number Variation in Patients with Hepatocellular Carcinoma by Next Generation DNA Sequencing. J Cancer, 6(3), 247-253. doi:10.7150/jca.10747

Xu, Y., Qi, Y., Rui, W., Zhu, Y., Zhang, C., Zhao, J., . . Ning, G. (2013). Expression and diagnostic relevance of heat shock protein 90 and signal transducer and activator of transcription 3 in malignant pheochromocytoma. J Clin Pathol, 66(4), 286-290. doi:10.1136/jclinpath-2012-201134

Yao, L., Barontini, M., Niederle, B., Jech, M., Pfragner, R., \& Dahia, P. L. (2010). Mutations of the metabolic genes IDH1, IDH2, and SDHAF2 are not major determinants of the pseudohypoxic phenotype of sporadic pheochromocytomas and paragangliomas. J Clin Endocrinol Metab, 95(3), 1469-1472. doi:10.1210/jc.2009-2245

Yao, L., Schiavi, F., Cascon, A., Qin, Y., Inglada-Perez, L., King, E. E., . . . Dahia, P. L. (2010). Spectrum and prevalence of FP/TMEM127 gene mutations in pheochromocytomas and paragangliomas. JAMA, 304(23), 2611-2619. doi:304/23/2611 [pii] 10.1001/jama.2010.1830

Ye, L., Santarpia, L., Cote, G. J., El-Naggar, A. K., \& Gagel, R. F. (2008). High resolution array-comparative genomic hybridization profiling reveals deoxyribonucleic acid copy number alterations associated with medullary thyroid carcinoma. J Clin Endocrinol Metab, 93(11), 4367-4372. doi:10.1210/jc.2008-0912

Yeap, P. M., Tobias, E. S., Mavraki, E., Fletcher, A., Bradshaw, N., Freel, E. M., . . Lindsay, R. S. (2011). Molecular Analysis of Pheochromocytoma after Maternal Transmission of SDHD Mutation Elucidates Mechanism of Parent-of-Origin Effect. J Clin Endocrinol Metab, 96(12), E2009-2013. doi:jc.2011-1244 [pii] 10.1210/jc.2011-1244

Yeh, I. T., Lenci, R. E., Qin, Y., Buddavarapu, K., Ligon, A. H., Leteurtre, E., .. . Dahia, P. L. (2008). A germline mutation of the KIF1B beta gene on $1 \mathrm{p} 36$ in a family with neural and nonneural tumors. Hum Genet, 124(3), 279-285. doi:10.1007/s00439-008-0553-1

Yokoyama, A., \& Cleary, M. L. (2008). Menin critically links MLL proteins with LEDGF on cancerassociated target genes. Cancer Cell, 14(1), 36-46. doi:S1535-6108(08)00158-X [pii] 10.1016/j.ccr.2008.05.003 
Yoshida, Y., Suzuki, K., Yamamoto, A., Sakai, N., Bando, M., Tanimoto, K., . . Nakamura, N. (2008). YIPF5 and YIF1A recycle between the ER and the Golgi apparatus and are involved in the maintenance of the Golgi structure. Exp Cell Res, 314(19), 3427-3443. doi:10.1016/j.yexcr.2008.07.023

Yoshimoto, K., Iwahana, H., Fukuda, A., Sano, T., Katsuragi, K., Kinoshita, M., . . . Itakura, M. (1992). ras mutations in endocrine tumors: mutation detection by polymerase chain reaction-single strand conformation polymorphism. Jpn J Cancer Res, 83(10), 1057-1062.

Zane, M., Agostini, M., Enzo, M. V., Casal Ide, E., Del Bianco, P., Torresan, F., . . Pelizzo, M. R. (2013). Circulating cell-free DNA, SLC5A8 and SLC26A4 hypermethylation, BRAF(V600E): A non-invasive tool panel for early detection of thyroid cancer. Biomed Pharmacother, 67(8), 723-730. doi:10.1016/j.biopha.2013.06.007

Zeiger, M. A., Zbar, B., Keiser, H., Linehan, W. M., \& Gnarra, J. R. (1995). Loss of heterozygosity on the short arm of chromosome 3 in sporadic, von Hippel-Lindau disease-associated, and familial pheochromocytoma. Genes Chromosomes Cancer, 13(3), 151-156.

Zhang, C., Shao, Y., Zhang, W., Wu, Q., Yang, H., Zhong, Q., . . Wan, J. (2010). High-resolution melting analysis of ADAMTS9 methylation levels in gastric, colorectal, and pancreatic cancers. Cancer Genet Cytogenet, 196(1), 38-44. doi:10.1016/j.cancergencyto.2009.08.016

Zhang, H., Gao, P., Fukuda, R., Kumar, G., Krishnamachary, B., Zeller, K. I., . . Semenza, G. L. (2007). HIF1 inhibits mitochondrial biogenesis and cellular respiration in VHL-deficient renal cell carcinoma by repression of C-MYC activity. Cancer Cell, 11(5), 407-420. doi:10.1016/j.ccr.2007.04.001

Zhang, J., Fujimoto, J., Zhang, J., Wedge, D. C., Song, X., Zhang, J., . . Futreal, P. A. (2014). Intratumor heterogeneity in localized lung adenocarcinomas delineated by multiregion sequencing. Science, 346(6206), 256-259. doi:10.1126/science.1256930

Zhuang, Z., Yang, C., Lorenzo, F., Merino, M., Fojo, T., Kebebew, E., . . Pacak, K. (2012). Somatic HIF2A gain-of-function mutations in paraganglioma with polycythemia. N Engl J Med, 367(10), 922930. doi:10.1056/NEJMoa1205119

Zuber, S., Wesley, R., Prodanov, T., Eisenhofer, G., Pacak, K., \& Kantorovich, V. (2014). Clinical utility of chromogranin A in SDHx-related paragangliomas. Eur J Clin Invest, 44(4), 365-371. doi:10.1111/eci.12245 